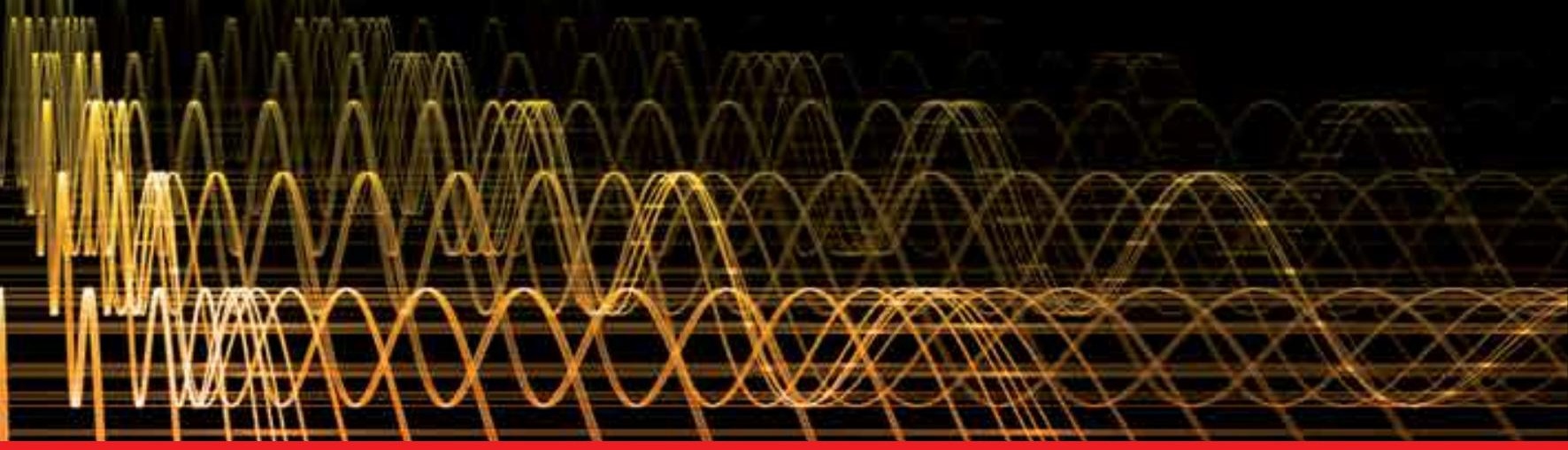

\title{
IntechOpen
}

\section{Energy Management of Distributed Generation Systems}

Edited by Lucian Mihet-Popa 



\section{ENERGY MANAGEMENT OF DISTRIBUTED GENERATION SYSTEMS}

Edited by Lucian Mihet-Popa 


\section{Energy Management of Distributed Generation Systems}

http://dx.doi.org/10.5772/61476

Edited by Lucian Mihet-Popa

\section{Contributors}

Hina Fathima, Kaliannan Palanisamy, Atanda Raji, Arno Vosloo, Jesús Rodríguez-Molina, Amjad Anvari-Moghaddam, Ghassem Mokhtari, Josep M. Guerrero, Ahmed Mohamed, Juan A. Martinez-Velasco, Gerardo Guerra, Rune Hylsberg Jacobsen, Søren Aagaard Mikkelsen, Shouxiang Wang, Leijiao Ge, Kai Wang, Shengxia Cai, Amjad Anvari-Moghadam, Ghavameddin Nourbakhsh, Bartlomiej Glowacki, Emma Sarah Hanley

\section{(c) The Editor(s) and the Author(s) 2016}

The moral rights of the and the author(s) have been asserted.

All rights to the book as a whole are reserved by INTECH. The book as a whole (compilation) cannot be reproduced, distributed or used for commercial or non-commercial purposes without INTECH's written permission.

Enquiries concerning the use of the book should be directed to INTECH rights and permissions department (permissions@intechopen.com).

Violations are liable to prosecution under the governing Copyright Law.

\section{(cc) BY}

Individual chapters of this publication are distributed under the terms of the Creative Commons Attribution 3.0 Unported License which permits commercial use, distribution and reproduction of the individual chapters, provided the original author(s) and source publication are appropriately acknowledged. If so indicated, certain images may not be included under the Creative Commons license. In such cases users will need to obtain permission from the license holder to reproduce the material. More details and guidelines concerning content reuse and adaptation can be foundat http://www.intechopen.com/copyright-policy.html.

\section{Notice}

Statements and opinions expressed in the chapters are these of the individual contributors and not necessarily those of the editors or publisher. No responsibility is accepted for the accuracy of information contained in the published chapters. The publisher assumes no responsibility for any damage or injury to persons or property arising out of the use of any materials, instructions, methods or ideas contained in the book.

First published in Croatia, 2016 by INTECH d.o.o.

eBook (PDF) Published by IN TECH d.o.o.

Place and year of publication of eBook (PDF): Rijeka, 2019.

IntechOpen is the global imprint of IN TECH d.o.o.

Printed in Croatia

Legal deposit, Croatia: National and University Library in Zagreb

Additional hard and PDF copies can be obtained from orders@intechopen.com

Energy Management of Distributed Generation Systems

Edited by Lucian Mihet-Popa

p. cm.

Print ISBN 978-953-51-2473-3

Online ISBN 978-953-51-2474-0

eBook (PDF) ISBN 978-953-51-6662-7 


\section{We are IntechOpen, the first native scientific \\ publisher of Open Access books}

$3,350+$

Open access books available

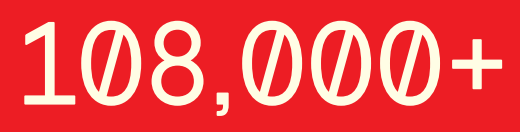

International authors and editors

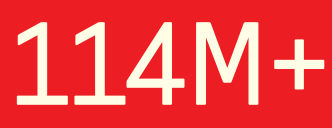

Downloads

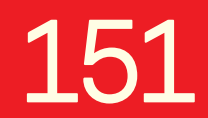

Countries delivered to

Our authors are among the

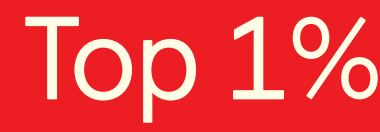

most cited scientists

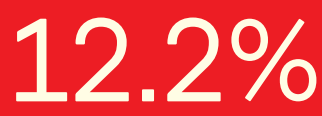

Contributors from top 500 universities

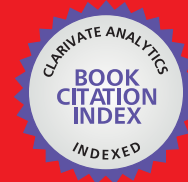

WEB OF SCIENCE ${ }^{\text {TM }}$

Selection of our books indexed in the Book Citation Index in Web of Science ${ }^{\mathrm{TM}}$ Core Collection (BKCI)

Interested in publishing with us?

Contact book.department@intechopen.com

Numbers displayed above are based on latest data collected.

For more information visit www.intechopen.com 



\section{Meet the editor}

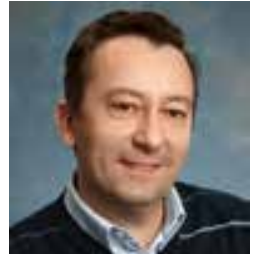

Dr. Lucian Mihet-Popa received the B.S. degree, M.S. degree, Ph.D. degree, and Dr. Habil from the Politehnica University of Timisoara, Timisoara, Romania, in 1999, 2000, 2003, and 2015, respectively, all in electrical engineering. He is currently working as an Associate Professor in the Department of Electrical Engineering at the Politehnica University of Timisoara. He published more than 80 papers, in national and international journals and conference proceedings, and 10 books. Dr. Mihet-Popa received the second prize paper award of the IEEE Industry Applications Society in 2005. His research interest includes modeling, simulations, control and testing of DER components in Smart Micro-Grids, electrical machines and drives, detection, and diagnosis of faults, especially for renewable conversion systems. 



\section{Contents}

Preface XI

Section 1 Distributed Control and Energy Management of Renewable Energy Sources 1

Chapter 1 Distributed Control and Management of Renewable Electric Energy Resources for Future Grid Requirements 3

Ghassem Mokhtari, Amjad Anvari-Moghaddam and Ghavameddin Nourbakhsh

Chapter 2 Coordinated Demand Response and Distributed Generation Management in Residential Smart Microgrids 27

Amjad Anvari-Moghaddam, Ghassem Mokhtari and Josep M.

Guerrero

Chapter 3 Hierarchical Control for DC Microgrids 59

Ahmed Mohamed

Section 2 Development of Control Algorithms, Software Architecture and Simulation Tools for Energy Management Systems 79

Chapter 4 Development of an Energy Management System Control Algorithm for a Remote Community Microgrid System 81 Arno Vosloo and Atanda K. Raji

Chapter 5 Allocation of Distributed Generation for Maximum Reduction of Energy Losses in Distribution Systems 107

Juan A. Martinez-Velasco and Gerardo Guerra

Chapter 6 The Role of Middleware in Distributed Energy Systems Integrated in the Smart Grid 133

Jesús Rodríguez-Molina 
Section 3 Energy Storage Systems Technology 155

Chapter 7 Energy Storage Systems for Energy Management of Renewables in Distributed Generation Systems 157 Amjed Hina Fathima and Kaliannan Palanisamy

Chapter 8 Energy Storage Technology for Decentralised Energy Management: Future Prospects 183

Bartek A. Glowacki and Emma S Hanley

Section 4 Energy Management Systems for Smart Homes 201

Chapter 9 Securing the Home Energy Management Platform 203

Søren Aagaard Mikkelsen and Rune Hylsberg Jacobsen

Section 5 Economical Optimization of Operational Cost for Micro-Grids 225

Chapter 10 Energy Management and Economic Operation Optimization of Microgrid under Uncertainty 227

Shouxiang Wang, Leijiao Ge, Kai Wang and Shengxia Cai 


\section{Preface}

Over the past decade, Energy Management of Distributed Generation Systems has been attracting an increasing interest and has become a major part of Smart (Micro)-Grids. The future Smart Grids with Energy Management Systems will include more Distributed Generation Systems, such as renewable energy sources, and also power electronics converters, energy storage technologies, advanced communications, and smart homes. On the edge of the Smart Grid infrastructure, where the consumers can produce energy from several local and distributed generation units, the need for energy management tools and control algorithms becomes an important option. To address these issues, many papers and books have proposed numerous software architectures and simulation models/tools.

The Smart Grids contain clusters of Micro-Grids, where generation, storage, and consumption take place. The new energy management systems will require different control paradigms that cope with increasing complexity of traditional power plants, with new energy conversion devices and systems, to ensure the Smart Grid systems' reliable operation, flexibility, and scalability.

Advanced distributed and decentralized transactive control structures are a feasible option for interconnected small-scale power plants and energy storage systems. Distributed controllers are particularly an attractive option to accommodate significant aggregation of renewable energy sources and active loads, such as smart homes and electric vehicles.

The objective of this book is to disseminate research and development results and to give an overview in the area of Energy Management of Distributed Generation using Renewable Energy Sources with actively controlled loads, such as Smart Homes, Electric Vehicles, and Energy Storage Systems. I would like to thank all authors and contributors of the individual chapters for their efforts and for the quality of the research material submitted. I would also like to thank Publishing Process Manager of this book for supporting this project and for a great support and collaboration during the publishing process.

The book contains 10 chapters, which is divided into five parts/sections. The first section entitled "Distributed Control and Energy Management of Renewable Energy Sources" including the first three chapters provides an overview of Energy Management of Distributed Systems, outlining the typical concepts, such as Demand-Side Management, Demand Response, Distributed, and Hierarchical Control for Smart Micro-Grids. The second section contains three chapters and presents different control algorithms, software architectures, and simulation tools dedicated to Energy Management Systems. In the third section, it is shown the importance and the role of energy storage technology in a Distribution System, describing and comparing different types of energy storage systems. The fourth section shows how to identify and address potential threats for a Home Energy Management Sys- 
tem. Finally, the fifth section discusses about Economical Optimization of Operational Cost for Micro-Grids, pointing out the effect of renewable energy sources, active loads, and energy storage systems on economic operation.

The first chapter, entitled "Distributed Control and Management of Renewable Electric Energy Resources for Future Grid Requirements" by Gassem Mokhtari et al., discusses some of the concepts used for distributed control, illustrating an application with case studies to support the method.

The second chapter, entitled "Coordinated Demand Response and Distributed Generation Management in Residential Smart Micro-Grids" by Amjad Anvari-Moghaddam et al., describes the functionality of a typical Demand-Side Management (DSM) strategy implemented on a building-level small-scale integrated energy system for a Residential Smart MicroGrid. An efficient framework for coordinated Demand Response and Distributed Energy Management into an integrated building and Smart Micro-Grid is outlined. The third chapter, entitled "Hierarchical Control for DC Micro-Grids" by Ahmed Mohamed, outlines aspects related to the design and control of DC Micro-Grids, pointing out the interaction of the Micro-Grid Central Controller with the controller of the main grid.

The second section "Development of Control Algorithms, Software Architectures, and Simulation Tools for Energy Management Systems" contains three chapters (Chapters 4-6). The fourth chapter, entitled "Development of an Energy Management System Control Algorithm for a Remote Community Micro-Grid System" by Arno Vosloo and Atanda K. Raji, points out the importance of controlling the output power of Micro-Grid components and the role of Energy Management for a Rural Micro-Grid System. The fifth chapter, entitled "Allocation of Distributed Generation for Maximum Reduction of Energy Losses in Distribution Systems" by Juan A. Martinez-Velasco and Gerardo Guerra, presents the importance of introducing powerful simulation tools with the applications of parallel computing methods, such as Parallel Monte Carlo approach, with respect to the allocation of Distributed Generation for maximum reduction of energy losses into Distribution Systems. The sixth chapter, entitled "The Role of Middleware in Distributed Energy Systems Integrated in the Smart Grid" by Jesus Rodriguez-Molina, deals with a software architecture for Smart Grids, which acts as a bridge between an operating system and real-time applications, pointing out the components and features of a Middleware architecture that works under the requirements and the study cases of a Smart Grid.

The third section "Energy Storage Systems Technology for Energy Management" contains two chapters. The focus of the seventh chapter, entitled "Energy Storage Systems for Energy Management of Renewable Energy Sources" by Ahmed H. Fathima and Palanisamy Kaliannan, is on the importance and the role of energy storage technology in a Distribution System, describing different types of energy storage systems and their applications related to Energy Management of integrated renewable systems. The eighth chapter, entitled "Energy Storage Technology and Decentralized Energy Management-Future Prospects" by Bartek A. Glowacki and Emma S. Hanley, evaluates and compares a few of energy storage technologies used to analyze Energy Management within the Decentralized Renewable and Storage Systems. Additionally, the integration of hydrogen storage technology and the use of hydrogen as an energy carrier in a decentralized airport scenario are highlighted.

The forth section "Energy Management Systems for Smart Homes" contains the ninth chapter "Securing the Home Energy Management Platform" by Soren Aagard and Jacobsen 
Rune. This chapter is trying to identify and address potential threats for a Home Energy Management System (HEMS) as a step in designing process. Based on the threats that have been detected, mitigation strategies are proposed, taking into account the state-of-the-art technology for securing platform of the HEMS.

The last section "Economical Optimization of Operational Cost for Micro-Grids" contains the 10th chapter "Energy Management and Economic Operation Optimization of Microgrid under Uncertainty" by Wang Shouxiang et al. The chapter proposes a Micro-Grid economic optimization model based on uncertainty characteristics of DER components, such as wind turbines and PV systems, and energy storage systems. Considering the effect of energy storage on economic operation, the chapter also discusses the impacts of uncertainties of renewable energy sources and loads on optimization model, as well as the effects of load fluctuation on scheduling results.

Associate Professor Lucian Mihet-Popa

Department of Electrical Engineering, Politehnica University of Timisoara, Romania 

Section 1

Distributed Control and Energy Management of Renewable Energy Sources 



\title{
Distributed Control and Management of Renewable Electric Energy Resources for Future Grid Requirements
}

\author{
Ghassem Mokhtari, Amjad Anvari-Moghaddam and \\ Ghavameddin Nourbakhsh
}

Additional information is available at the end of the chapter

http://dx.doi.org/10.5772/63378

\begin{abstract}
It is anticipated that both medium- and low-voltage distribution networks will include high level of distributed renewable energy resources, in the future. The high penetration of these resources inevitably can introduce various power quality issues, including; overvoltage and overloading. This book chapter provides the current research state of the art concepts and techniques in dealing with these potential issues. The methods provided in this chapter are based on distributed control approach, tailored and suitable particularly for the future distribution composition. The distributed control strategy is a promising approach to manage and utilise the resources in future distribution networks to effectively deal with grid electric quality issues and requirements. Jointly, utility and customers the owners of the resources in the network are considered as part of a practical coordination strategy in this method. Standard IEEE test system is used for application, and to demonstrate the effectiveness of the method by providing the results.
\end{abstract}

Keywords: distributed control, consensus algorithm, energy storage system (ESS), future grid requirements, network overloading

\section{Introduction}

Distribution networks are usually designed to provide electric power to the customers, while operating within the grid national electricity standards, in particular having voltage and loading in permissible range. The existing distribution network planning, design and operation usually does not consider the existence of renewable energy sources such as 
Photovoltaic (PV) and wind turbine [1, 2]. Future distribution networks with high utilisation of these resources will have to meet certain requirements addressing issues such as voltage and equipment loading $[2,3]$. Therefore, new facilities, methods and strategies have to be envisaged for proper design, operation and planning of future distribution networks.

Literature offers numerous methods and strategies that are designed to deal with the future network requirements. Resources such as renewable energy sources, storage systems and smart loads with practical and adequate control methods will play an important role in this regard. These resources can be utilised for various reasons by utilities and customers [4]. Among many utilisation of these facilities, network loading support is the main application addressed in this chapter. Due to the variety use of these resources, normally supervisory control could not deliver promising objectives due to the reliability and complexity issues [2]. On the other hand, neither local management approach could offer an efficient control strategy [2]. However, distributed control is found to be the most practical control strategy in application to future smart grid [1], particularly for distribution network [2]. This control approach has already been established for many applications as reported in recent research citations [37]. To have a distributed management of resources, the smart network usually includes two layers. The first layer as physical layer consist of grid lines and energy resources, while the second layer as the cyber layer is added to provide information exchange between the nodes. Distributed control strategy uses the immediate neighbouring communication information to control and manage energy resources.

The main aim of this book chapter is to provide some of the concepts and formulations used for distributed control, and to illustrate an application with results to support the method. In this approach, energy storage system (ESS) is considered as the promising facility for customers and utilities that can be used for different applications. Loading issue is considered as one of the network requirement which need to be dealt with using ESSs in distribution network. For all practical purposes, utility and/or customer are considered as the owner of storage facilities.

Section 2 discusses the main technical issues and influencing factors relating to the future state of distribution network operation with renewable energy resources. Distributed control management and its application in distribution network are put in perspective in Section 3. Distributed management of energy resources based on specific network requirement is discussed in Section 4. A case study using the distributed control strategy with detailed results is provided in Section 5. Finally, Section 6 concludes this chapter with related discussions and conclusions.

\section{Future state of distribution networks with renewable energy resources}

\subsection{Power quality issues associated with high utilisation of renewable energy sources}

Renewable energy sources will play an important role in future electricity grid. Depending on the geographical location and network structure, they may be in either off-grid or grid- 
connected situation. Despite the mode of network connection, economically speaking, the main purpose of these resources was to reduce electricity consumption cost. However, from the technical point of view, there is a difference between these two connecting modes. In offgrid mode, usually the main aim was to balance generation and load to avoid load outage, which is not desirable [8]. However, in grid-connected mode, as grid support is available, the unbalancing can be tolerated to some allowable extend. However, significant unbalancing between generation and load can induce power quality issues. To better understand these issues, Figure 1 shows a single-line diagram of distribution network in which all customers' load and generation in each phase are modelled by single generation and load. Based on this model, different issues associated by renewable energy sources can be addressed as follow:

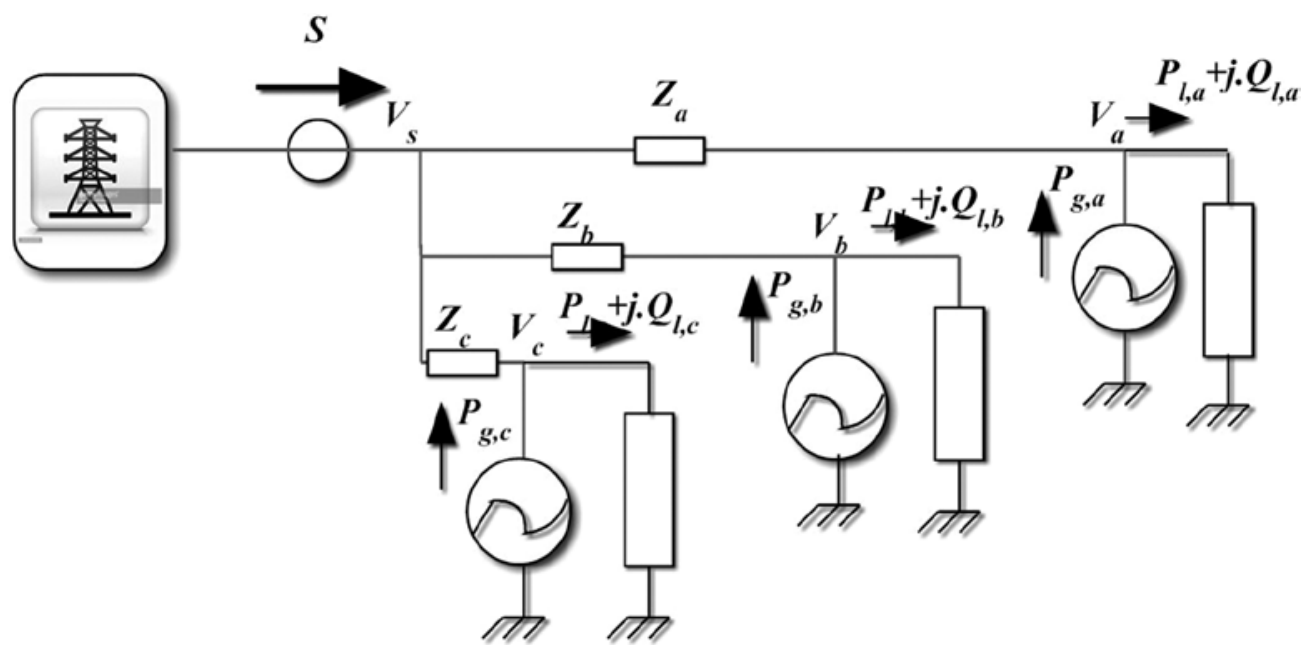

Figure 1. Distribution network equivalent, with renewable energy sources.

\subsubsection{Voltage rise}

Voltage rise due to high penetration of renewable energy sources is the main power quality issue in future distribution network. In high generation mode, as the load is usually in offpeak mode, there is an unbalancing between generation and load. This unbalancing can cause voltages to rise. To see this impact, let us have a close look at Figure 1. Based on this figure, the voltages magnitude for end of each phase can be written as [9]:

$$
\begin{aligned}
& \left|V_{a}\right|=\left|V_{s}\right|+\frac{\left(P_{g, a}-P_{l, a}\right) \cdot R_{a}-Q_{l, a} \cdot X_{l, a}}{\left|V_{s}\right|} \\
& \left|V_{b}\right|=\left|V_{s}\right|+\frac{\left(P_{g, b}-P_{l, b}\right) \cdot R_{b}-Q_{l, b} \cdot X_{l, b}}{\left|V_{s}\right|}
\end{aligned}
$$




$$
\left|V_{c}\right|=\left|V_{s}\right|+\frac{\left(P_{g, c}-P_{l, c}\right) \cdot R_{c}-Q_{l, c} \cdot X_{l, c}}{\left|V_{s}\right|}
$$

where

$P_{g a} P_{g b}$ and $P_{g c}$ are the injected active power in each phase,

$P_{l a} P_{l b}$ and $P_{l c}$ are the consumed active power in each phase,

$Q_{l a r} Q_{l b}$ and $Q_{l c}$ are the reactive power in each phase,

$R_{a}, R_{b}$ and $R_{c}$ are the line resistance of each phase,

$X_{a}, X_{b}$ and $X_{c}$ are the line reactance of each phase.

It can be seen that when a phase source active power is more than the load in that phase, the phase voltage will increase. If this unbalancing is considerably high, a stationary limit can be violated which is not acceptable by regulations and standards.

\subsubsection{Voltage unbalancing}

Voltage unbalancing is happening when there are differences between the three phase voltage magnitudes and/or angles (not separated by $120^{\circ}$ ). Based on Eqs. (1)-(3), as the loading and generation of a phase changes with respect to other phases, the magnitudes or angles of $V_{a}, V_{b}$ and $V_{c}$ will be unbalanced. Therefore, it can be seen that the voltage unbalancing is a common power quality issue in distribution network. The voltage unbalancing is usually measured by voltage unbalancing factor (VUF) as in Eq. (4) [10].

$$
\% V U F=\frac{\left|V_{-}\right|}{\left|V_{+}\right|} \times 100
$$

where $V_{-}$and $V_{+}$are the negative and positive sequence voltage components which can be calculated as follow:

$$
\begin{aligned}
& V_{-}=\frac{V_{a}+a \cdot V_{b}+a^{2} \cdot V_{c}}{3} \\
& V_{+}=\frac{V_{a}+a^{2} \cdot V_{b}+a \cdot V_{c}}{3}
\end{aligned}
$$

where

$$
a=1<120 \text { and } a^{2}=1<240 \text {. }
$$


Based on national standards, usually up to a maximum VUF of $2 \%$ is accepted in distribution network [11].

\subsubsection{Harmonic}

As most renewable energy sources are connected through power electronic converter to the distribution network, they usually inject harmonics to the network. Total Harmonic Distortion (THD) is usually considered as the harmonic index which normally need to be $<5 \%$ based on standards [12].

\subsubsection{Frequency variation}

Frequency deviation can also result from unbalancing between load and generation. During peak generation period, when generation is more than load, the frequency may rise. Additionally, during peak load period, the load is higher than generation which may cause the frequency to drop.

\subsubsection{Loading constraint violation}

Based on Figure 1, the load of each phase and power transformer can be written as follows:

$$
\begin{gathered}
\left|S_{a}\right|=\sqrt{\left(P_{l, a}-P_{g, a}\right)^{2}+Q_{l, a}^{2}} \\
\left|S_{b}\right|=\sqrt{\left(P_{l, b}-P_{g, b}\right)^{2}+Q_{l, b}^{2}} \\
\left|S_{c}\right|=\sqrt{\left(P_{l, c}-P_{g, c}\right)^{2}+Q_{l, c}^{2}} \\
S=S_{a}+S_{b}+S_{c}
\end{gathered}
$$

where $S_{a} S_{b}$ and $S_{c}$ are the apparent power of each phase and $S$ is the apparent power of power transformer.

Based on these equations, it can be seen that the loading of lines and power transformer depends on the difference between generation and load in each phase. If this difference is high, it can violate some of the facilities' thermal limit.

In this chapter, this issue is considered as one of the requirements for distribution network operation, which need to be dealt with using available resources in the network. 


\subsection{Coordinating network resources based on network requirements}

Future distribution networks will include a lot of controllable resources which can be used and coordinated based on different network requirements. This section lists some of the resources, which can be utilised based on network requirements.

\subsubsection{Source active power}

Injecting surplus active power to the grid by renewable energy source is the main cause of power quality issues. Therefore, one way to deal with these issues is to control the injecting power. For instance, Refs. $[7,9,13]$ use active power curtailment as a robust control approach to deal with voltage rise issue.

\subsubsection{Source reactive power}

Another resource which can be used to deal with power quality issues in distribution network is reactive power contribution of renewable energy sources. This strategy is usually used to deal with voltage fluctuation in distribution network. References [14-16] use reactive power control in distribution network to deal with voltage rise.

\subsubsection{Controllable load}

To deal with the unbalance between load and generation, smart controllable loads such as airconditioner or washing machine are suitable options which can be utilised to deal with power quality issues as well [17]. These resources can be used for both voltage and loading support in network. References [13, 16] use these resources to prevent overvoltages in distribution network.

\subsubsection{Energy storage system (ESS)}

ESS has an important role in developing future smart grid [18] which appears in several types such as flywheel, super capacitor, compressed gas and battery. Battery is the most popular ESS in distribution network which can be considered as the suitable option for customers or utilities application based on network requirements. This facility can be charged during high generation period, while discharging during peak load period. Therefore, it can easily reduce the unbalancing between generation and loading, while dealing with network issues.

Based on the versatility and vital role of batteries in future distribution grids, storage has been included in this chapter as part of the strategy and application for load and renewable energy management, while considering its role in resolving network quality issues for future distribution systems. 


\section{Distributed control management and its application in distribution network}

Distributed control is an effective management approach for future smart grids with distributed resources. This approach has been applied in various literatures in recent years. There are two comprehensive review papers which study the application of distributed control in power systems. Reference [19] presented different types of distributed multi-agent systems and their applications in power systems. In Ref. [20], the applications of distributed control in micro-grids are studied which includes; primary control, voltage coordination, economic dispatch and frequency control. In this study, distributed control are categorised based on problem formulisation into three main categories [20]:

- Predictive control-based approach,

- Agent-based approach,

- Consensus-based approach.

Consensus-based approach is a new distributed control approach which aims to have an equal proportionality-based converging sate for the resources. In Ref. [2], this approach is used to deal with network loading, while coordinating storage units in a fair way. Reference [7] adapted an overvoltage control approach based on this algorithm which uses fair battery charging to prevent voltage rise in low voltage network.

Consensus algorithm also applied in recent studies to provide optimal utilisation of resources as well. In [8], a new distributed updating approach is used to utilise batteries in an efficient way in micro-grid. A new distributed optimal control approach is proposed in [21] which manages multiple generators based on consensus algorithm. It can be seen that the consensusbased distributed approach has attracted a lot of studies in recent years. This book chapter includes the use of this algorithm as the backbone of distributed management of resources in distribution network. The model for this algorithm is as follow:

In consensus algorithm, network is modelled with graph $G$ having $N$ vertices. The graph is shown by $G(V, E)$ where $V$ is the set of vertices and $E$ is the set of edges. For this graph, $(i, j) \in E$ if node $i$ can receive information from node $j$. This communication link is shown by $c_{i j}$ in which the value of 1 for this parameter means there is a communication link and 0 means no communication. Additionally, neighbours of node $i$ are those nodes which send information to node $i$ which are shown by set of $N_{i}=\{j \in V,(i, j) \in E\}[6]$.

In consensus algorithm, a parameter named as information state is defined for each resource. Based on this algorithm, the information state of each resource is a function of information state of its neighbours which can be shown as in Eq. (11) [6].

$$
\varepsilon_{i}[t]=\sum_{j \in N_{i}} d_{i j} \varepsilon_{j}[t]
$$


where

$\varepsilon_{i}(t)$ is the information state of $i$ th resource and;

$$
d_{i j}=\frac{c_{j i}}{\sum_{k \in N_{i}} c_{k i}}
$$

\section{Distributed management of energy resources based on specific network requirement}

As noted before, distributed management approach has been applied in variety of power system applications. In this section, the aim was to apply distributed control approach for specific application, which is distributed loading management in distribution network. Battery as an ESS with its associated inverter is considered as the main resource which can be used for this purpose.

If there is only one single ESS in distribution network, there is no need to apply any coordination strategy. However, future distribution network may include high number of ESSs; therefore, a coordination strategy is needed to coordinate these units based on specific objective function.

The proposed control structure for distribution network which coordinates multiple ESSs is shown in Figure 2. To manage multiple ESSs in a distributed way, a communication link is assumed between neighbouring ESSs. Additionally, there is a control agent named as the leader which monitors network loading and initiates the ESS coordination whenever it is needed.

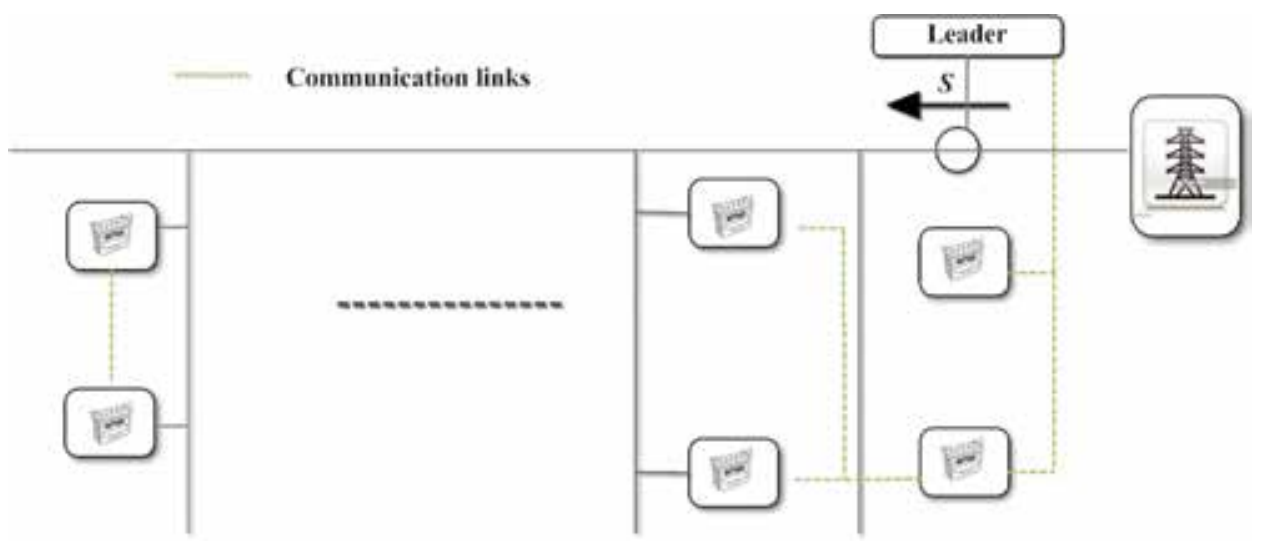

Figure 2. Distributed control structure for distribution network. 
To coordinate the ESSs for loading management, three operating control modes are considered for the network. These network control modes are shown in Figure 3, which there are four limits that determine the network control modes. If the network loading is within desirable range ( $S_{d}^{\text {gen. }}$ and $S_{d}^{\text {cons. }}$ ), the network is in normal condition. Therefore, there is no need for ESSs' coordination based on network requirements and they can operate based on other objectives. If network loading violates $S_{c}{ }^{\text {gen. }}$, the network goes to the high generation control mode. In this control mode, ESSs should be coordinated to charge and reduce the network loading. Additionally, if the network loading violates $S_{c}^{\text {cons. }}$, the network goes to the high consumption mode which means that the ESSs should be coordinated to discharge and reduce the network loading.

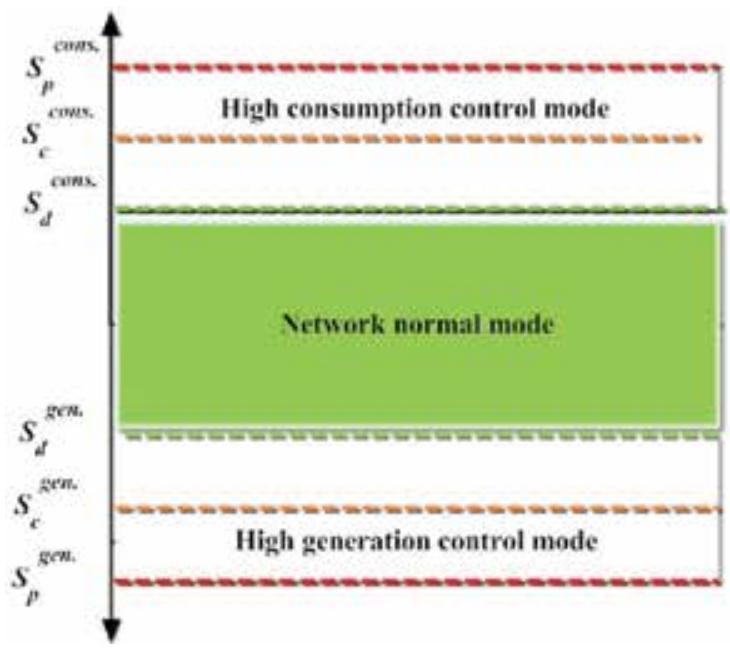

Figure 3. Network control modes based on predefined limits.

Based on consensus algorithm in this section, two distributed management approaches are proposed to coordinate multiple ESSs for loading management. Each distributed approach follows a specific objective function.

To find these objective functions, the ESS's owner interest is considered. If the ESS owners are customers, their preference is assumed to have a fair contribution in loading management. In other words, all customers prefer to have a fair sharing in loading management. However, for utility owned EES, the aims normally were to maximise the ESSs' utilisation of these devices. Therefore, it can be said that a proper coordination should be considered for each of these objectives. The details of the two distributed control approaches which consider each of these objectives to coordinate multiple ESSs are as follow:

\subsection{Network loading management using fair sharing of multiple ESSs}

In this case, the objective function includes two main parts [2]. To keep the network loading within acceptable range, the first objective was defined by keeping the network loading between critical limits: 


$$
S_{c}^{\text {gen. }} \leq S \leq S_{c}^{\text {cons }}
$$

Additionally, to have a fair sharing among multiple ESSs, the second objective was defined by having the following ratio

$$
\frac{P_{1}}{P_{1}^{\max }}=\frac{P_{2}}{P_{2}^{\max .}}=\ldots=\frac{P_{n}}{P_{n}^{\max }}
$$

where

$P_{1}^{\text {max. }}$ is the maximum active power available $i$ th ESS which depends on its state-of-charge (SOC). The objective can be also rewritten as follow:

$$
\frac{P_{1}}{S O C_{1}}=\frac{P_{2}}{S O C_{2}}=\ldots=\frac{P_{n}}{S O C_{n}}
$$

To achieve the noted objectives, the control structure of the leader, shown in Figure 4, will be used. In other words, as soon as the critical limits for generation or consumption are violated, the leader starts to update its information state as given in Eqs. (16) and (17).

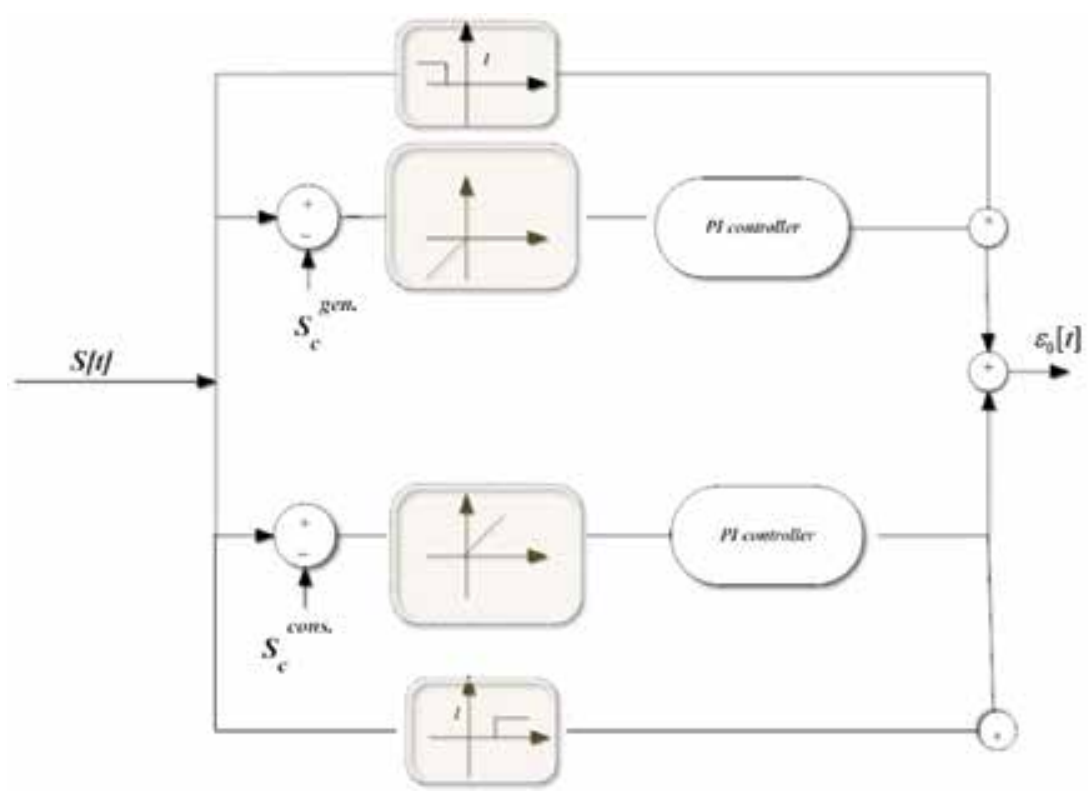

Figure 4. Leader control structure for ESS fair contribution. 


$$
\begin{aligned}
& \varepsilon_{0}[t]=\varepsilon_{0}\left[t-t_{s}\right]+k \cdot\left(S[t]-S_{c}^{g e n .}\right) \\
& \varepsilon_{0}[t]=\varepsilon_{0}\left[t-t_{s}\right]+k \cdot\left(S[t]-S_{c}^{c o n s .}\right)
\end{aligned}
$$

Once the leader initiates the control, the ESSs will update their information state based on consensus algorithm, as given in Eq. (18).

$$
\varepsilon_{i}[t]=\sum_{j \in N_{i}} d_{i j} \varepsilon_{j}[t]
$$

Based on the calculated ESS state information, the reference for ESS active power will be updated as in Eq. (19).

$$
P_{i}[t]=\varepsilon_{i}[t] \cdot P_{i}^{\max } .
$$

The flowchart of this distributed loading control management is shown in Figure 5.

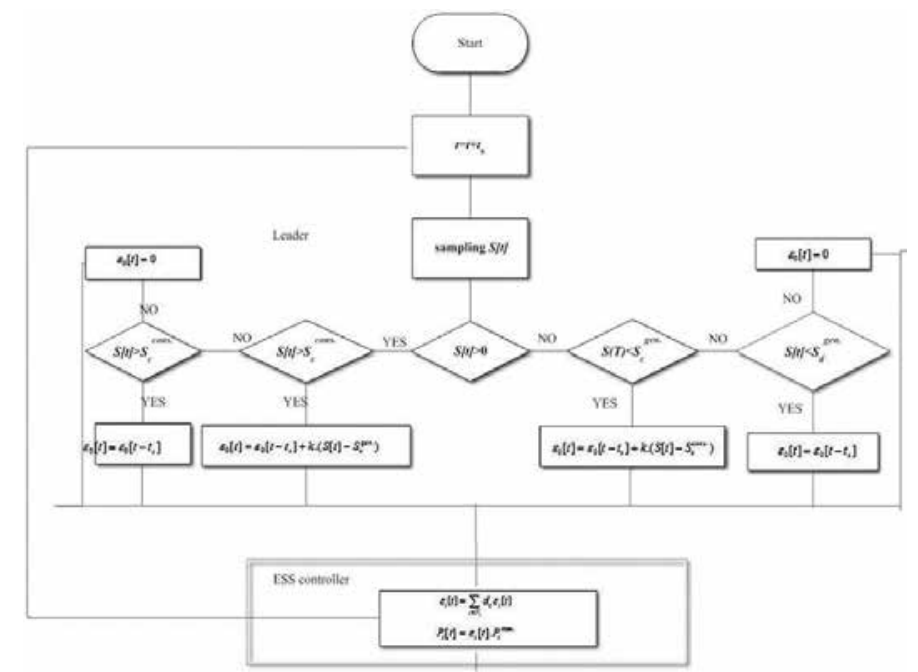

Figure 5. Distributed control structure for fair sharing of multiple ESSs.

\subsection{Network loading management using optimal utilisation of multiple ESSs}

In this case, the interest of utility is considered in managing multiple ESSs. In other words, the aim was to use ESSs for loading management while the utilisation rate and efficiency of the 
batteries are maximised. To achieve such an objective, the cost function given in Eq. (20) is used for each storage unit [22].

$$
C_{i}\left(\left|P_{i}\right|\right)=\eta_{i} \cdot\left|P_{i}\right|=a_{i} \cdot\left|P_{i}\right|-b_{i} \cdot\left|P_{i}\right|^{2}
$$

where

$\eta_{i}$ is the efficiency of ESS in charging and discharging mode (it is assumed to be the same in both mode).

$a_{i}$ and $b_{i}$ are the efficiency coefficient of ESS.

In this case, the goal was to maximise this cost function while reduce loading to less than the critical limits. For instance, in high generation control mode, the cost function can be written as follows:

$$
\begin{aligned}
& \operatorname{Max} \sum_{i=1}^{N_{s}} C_{i}\left(\left|P_{i}\right|\right) \\
& \sum_{i=1}^{n}\left|P_{i}\right|=\left|S-S_{c}^{\text {gen. }}\right| \\
& P_{i}^{\text {min. }} \leq P_{i} \leq P_{i}^{\text {max. }}
\end{aligned}
$$

Note that the same cost function can be used for high loading control mode, and only the equality constraint will change.

Based on central optimisation approach, the optimal point of incremental cost is as follows

$$
\varepsilon^{*}=\frac{\left(\sum_{i=1}^{n} \frac{a_{i}}{2 b_{i}}-\left|S-S_{c}^{g e n .}\right|\right)}{\sum_{i=1}^{n} \frac{1}{2 b_{i}}}
$$

To converge the optimal point and maximise cost function in Eq. (21), the iterative updating approach of the following equations is used [8]:

$$
\begin{gathered}
\varepsilon_{i}\left[t+t_{s}\right]=\sum_{j \in N_{i}} d_{i j} \cdot \varepsilon_{j}[t]+\alpha \cdot P_{l, i}(t) \\
\left|P_{i}\left[t+t_{s}\right]\right|=\left(\frac{-\varepsilon_{i}\left(t+t_{s}\right)+a_{i}}{2 . b_{i}}\right)
\end{gathered}
$$




$$
\begin{gathered}
P_{l, i}^{\prime}\left[t+t_{s}\right]=P_{l, i}[t]+\left(\left|P_{i}\left(t+t_{s}\right]\right|-\left|P_{i}(t]\right|\right) \\
P_{l, i}\left[t+t_{s}\right]=\left(\sum_{j \in N_{i}} d_{i j} P_{l, j}^{\prime}[t]\right) \\
\operatorname{flag}_{i}[t]=\operatorname{flag}_{i-1}\left[t-t_{s}\right] \\
P_{i}[t]=\operatorname{flag}_{i}[t] .\left|P_{i}[t]\right|
\end{gathered}
$$

If this iterative process is initiated by leader using Eqs. (29) and (30) when the critical limit is violated;

$$
\begin{gathered}
\operatorname{flag}_{0}[t]=-1 \\
P_{l, i}(t)=\left\{\begin{array}{cc}
S-S_{c}^{\text {gen. }} & i=1 \\
0 & i \neq 1
\end{array}\right.
\end{gathered}
$$

Based on Ref. [8], the Eqs. (23) and (24) converge to the following:

$$
\begin{gathered}
\varepsilon[\infty]=\left[\begin{array}{llll}
\varepsilon^{*} & \varepsilon^{*} & \ldots & \varepsilon^{*}
\end{array}\right]_{n}=\varepsilon^{*} \cdot 1_{n} \\
\sum_{i=1}^{n}\left|P_{l, i}(0)\right|=-\sum_{i=1}^{n} \frac{1}{2 . b_{i}} \cdot \varepsilon^{*}+\sum_{i=1}^{n} \frac{a_{i}}{2 b_{i}}
\end{gathered}
$$

Therefore, it can be seen that the incremental cost converge to the noted optimal point, as given in Eq. (33)

$$
\varepsilon^{*}=\frac{\left(\sum_{i=1}^{n} \frac{a_{i}}{2 b_{i}}-\left|S-S_{c}^{\text {gen. }}\right|\right)}{\sum_{i=1}^{n} \frac{1}{2 b_{i}}}
$$

The flowchart of this distributed loading control management approach is shown in Figure 6. 


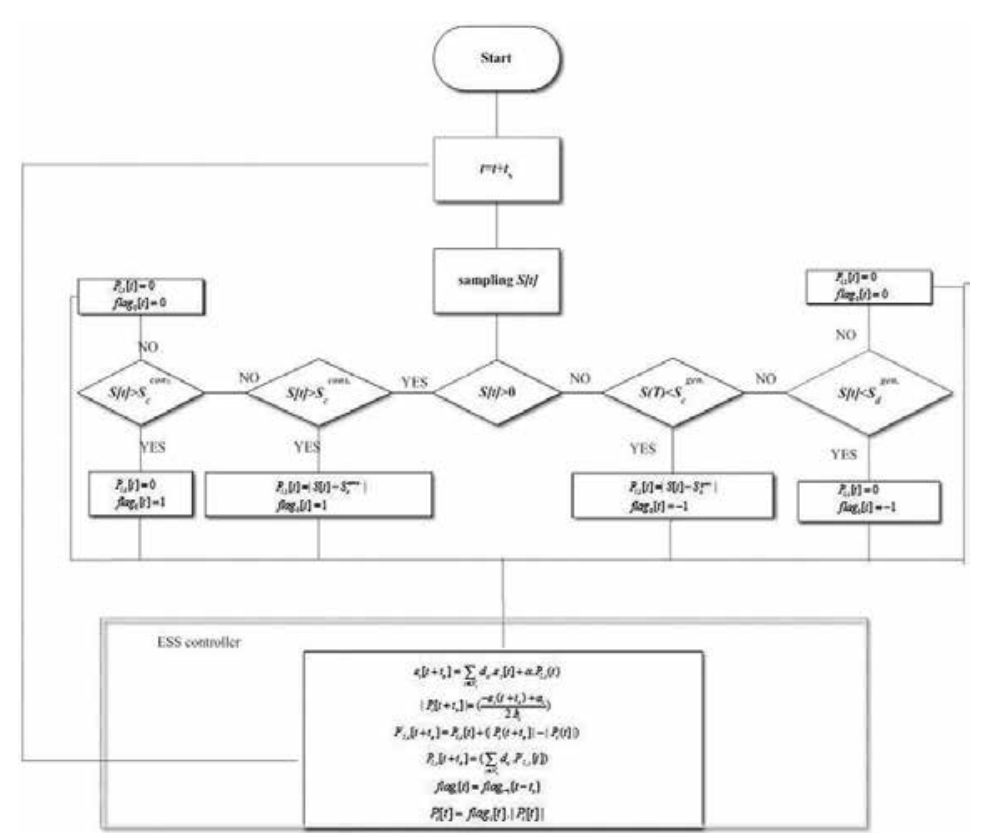

Figure 6. Distributed control structure for optimal utilisation of multiple ESSs.

\section{Case studies}

In this section, IEEE 33-bus distribution system is used to assess the performance of the distributed loading management approaches [23]. The details of ESSs are shown in Table 1. All the buses assumed to have their peak generation the same as their peak load. The aim of this section was to coordinate multiple ESSs to manage the loading of this network within -3000 to $3000 \mathrm{kVA}$ as given in Table 2. To assess the performance of this approach in different network modes, the loading and generation profiles for each bus given in Figure 7 are considered. The profiles are based on maximum loading and generation at each bus. MATLAB platform is used to implement the proposed approaches in the following case studies.

\begin{tabular}{lllllllll}
\hline ESS & $\mathbf{1}$ & $\mathbf{2}$ & $\mathbf{3}$ & $\mathbf{4}$ & $\mathbf{5}$ & $\mathbf{6}$ & $\mathbf{7}$ & $\mathbf{8}$ \\
\hline Location (bus) & 2 & 8 & 12 & 15 & 20 & 27 & 30 & 33 \\
Rating $(\mathrm{kW})$ & 150 & 160 & 120 & 180 & 100 & 140 & 150 & 140 \\
$a_{i}$ & .91 & .9 & .89 & .9 & .9 & .92 & .9 & .9 \\
$b_{i}$ & .09 & .05 & .12 & .12 & .13 & .09 & .08 & .1 \\
\hline
\end{tabular}

Table 1. ESSs parameters. 


\begin{tabular}{ll}
\hline Parameter & Power (kVA) \\
\hline$S_{p}^{\text {cons. }}$ & 3200 \\
$S_{c}^{\text {cons. }}$ & 3000 \\
$S_{d}^{\text {cons. }}$ & 2800 \\
$S_{d}^{\text {gen. }}$ & -2800 \\
$S_{c}^{\text {gen. }}$ & -3000 \\
$S_{p}^{\text {gen. }}$ & -3200 \\
\hline
\end{tabular}

Table 2. Loading limits in the proposed approach.

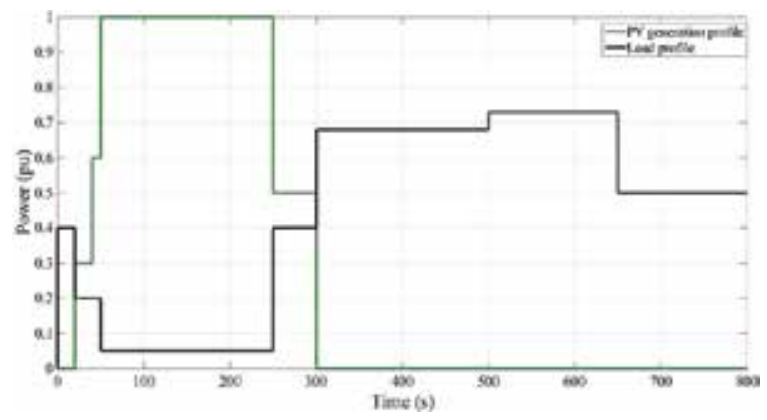

Figure 7. Load and generation profiles at each bus.

\subsection{Case 1}

The aim of this case study was to coordinate multiple ESSs in a fair way, while managing the network loading. The results for distributed loading management using this approach are shown in Figure 8. As it can be seen, the system goes to the high generation control mode at $t$ $=50 \mathrm{~s}$ and multiple ESSs reduce the power injected to the upper level grid $<S_{c}{ }_{c}{ }^{e n}$. The contribution of each ESS at steady state is listed in Table 3, which follows the fair sharing objective as follow:

$$
\frac{P_{1}}{P_{1}^{\max }}=\frac{P_{2}}{P_{2}^{\max .}}=\ldots=\frac{P_{8}}{P_{8}^{\max }}=-0.344
$$

The same scenario is happening at $t=500 \mathrm{~s}$ in which the ESSs goes to the high consumption control mode, with ESS contribution for loading management in a fair way as given in following:

$$
\frac{P_{1}}{P_{1}^{\max .}}=\frac{P_{2}}{P_{2}^{\max .}}=\ldots=\frac{P_{8}}{P_{8}^{\max .}}=0.158
$$



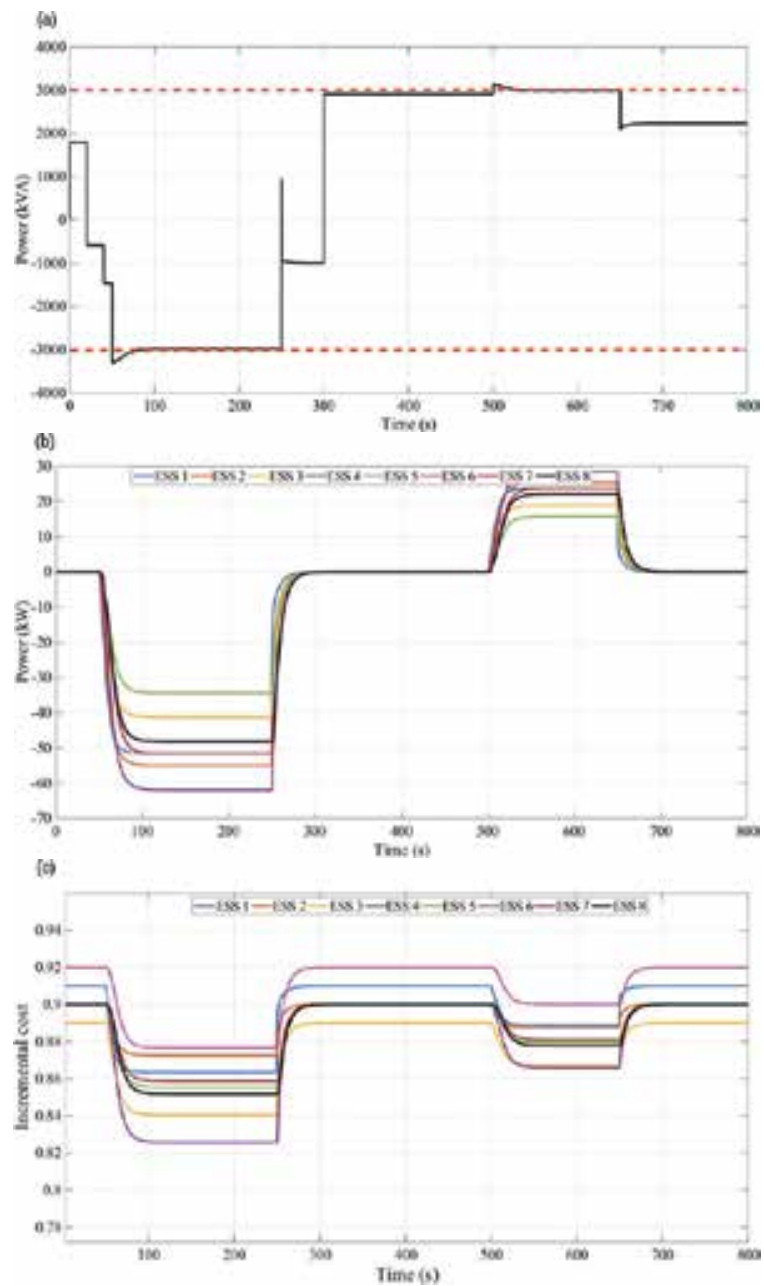

Figure 8. Results for fair sharing among ESSs (a) network loading, (b) ESS contribution, (c) incremental cost.

\begin{tabular}{llllllllll}
\hline ESS & $\mathbf{1}$ & $\mathbf{2}$ & $\mathbf{3}$ & $\mathbf{4}$ & $\mathbf{5}$ & $\mathbf{6}$ & $\mathbf{7}$ & $\mathbf{8}$ & Total \\
\hline $0-50 \mathrm{~s}$ & 0 & 0 & 0 & 0 & 0 & 0 & 0 & 0 & 0 \\
$50-250 \mathrm{~s}$ & 0 & 0 & 0 & 0 & 0 & 0 & 0 & 0 & 0 \\
$50-250 \mathrm{~s}$ & -51.631 & -55.073 & -41.304 & -61.957 & -34.420 & -48.188 & -51.631 & -48.188 & -392.36 \\
$250-500 \mathrm{~s}$ & 0 & 0 & 0 & 0 & 0 & 0 & 0 & 0 & 0 \\
$500-650 \mathrm{~s}$ & 23.698 & 25.278 & 18.958 & 28.437 & 15.798 & 22.118 & 23.698 & 22.118 & 157.93 \\
$650-800 \mathrm{~s}$ & 0 & 0 & 0 & 0 & 0 & 0 & 0 & 0 & 0
\end{tabular}

Table 3. ESSs power contributions for loading management. 


\subsection{Case 2}

In this case, the aim was to coordinate multiple ESSs, while optimising their utilisation rate and maximise the cost function in Eq. (21). The results for this case are shown in Figure 9. The ESSs' contributions for each time step are listed in Table 4. It can be seen that the ESSs with lower cost coefficients contribute more in loading management. Additionally, the total ESSs contribution in each time step is less than the previous case which shows the advantage in promising features of this optimal approach.
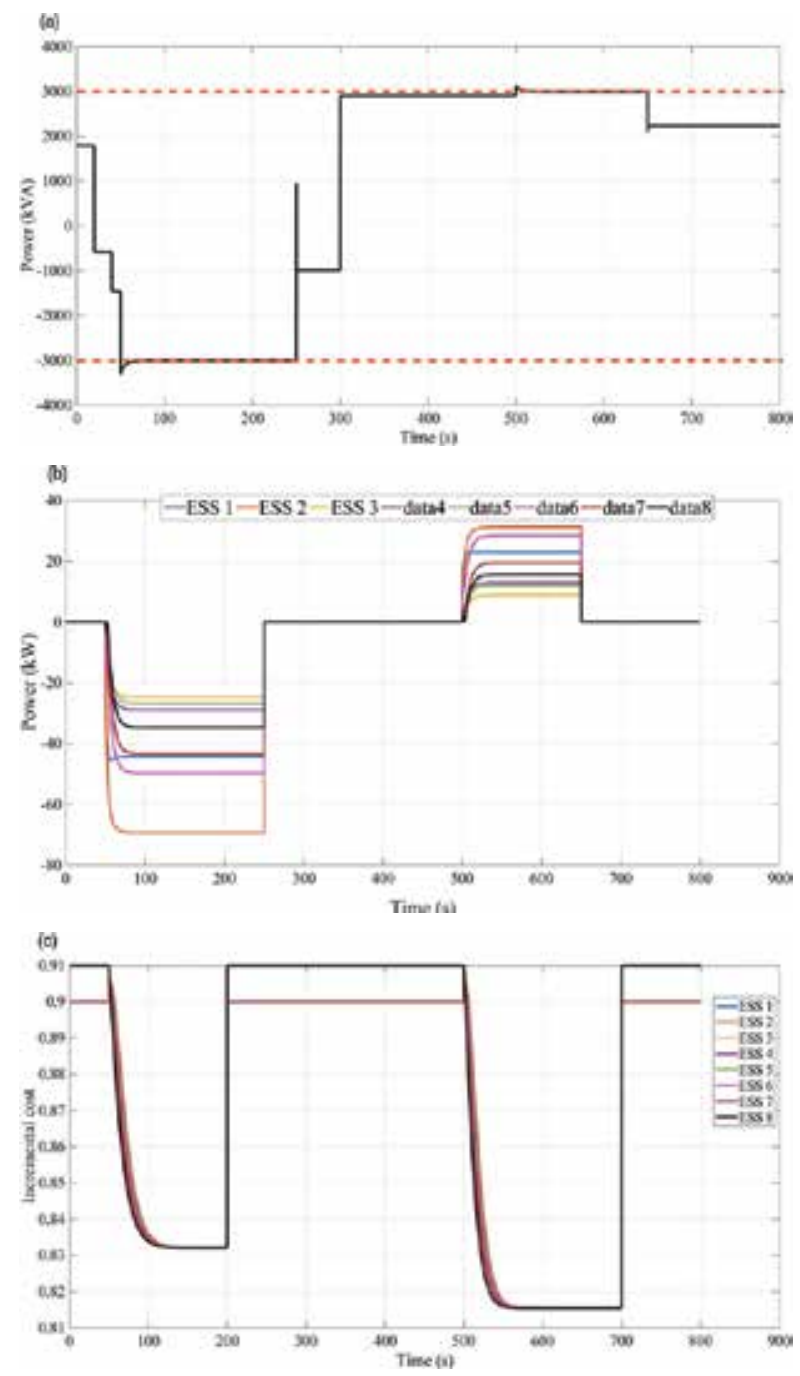

Figure 9. Results for optimal ESSs coordination, (a) network loading, (b) ESS contribution, (c) incremental cost. 


\begin{tabular}{llllllllll}
\hline ESS & $\mathbf{1}$ & $\mathbf{2}$ & $\mathbf{3}$ & $\mathbf{4}$ & $\mathbf{5}$ & $\mathbf{6}$ & $\mathbf{7}$ & $\mathbf{8}$ & Total \\
\hline $0-50 \mathrm{~s}$ & 0 & 0 & 0 & 0 & 0 & 0 & 0 & 0 & 0 \\
$50-250 \mathrm{~s}$ & 0 & 0 & 0 & 0 & 0 & 0 & 0 & 0 & 0 \\
$50-250 \mathrm{~s}$ & -44.12 & -69.41 & -24.75 & -28.92 & -26.70 & -49.67 & -43.38 & -34.70 & -321.65 \\
$250-500 \mathrm{~s}$ & 0 & 0 & 0 & 0 & 0 & 0 & 0 & 0 & 0 \\
$500-650 \mathrm{~s}$ & 22.96 & 31.33 & 8.89 & 13.05 & 12.05 & 28.52 & 19.58 & 15.67 & 152.05 \\
$650-800 \mathrm{~s}$ & 0 & 0 & 0 & 0 & 0 & 0 & 0 & 0 & 0 \\
\hline
\end{tabular}

Table 4. ESSs power contributions for loading management.

\subsection{Case 3}

Finally, in this case, the impact of communication drop is studied on the distributed loading management approaches. To model this impact, it is assumed that the communication links between ESS 4 and 5 are unavailable during $t=450-600 \mathrm{~s}$ as given in Eq. (34).

$$
c_{45}=c_{54}=\left\{\begin{array}{cc}
1 & t<450 \quad t>600 \\
0 & 450 \leq t \leq 600
\end{array}\right.
$$

The results for both scenarios are provided as follow. For the first case in which ESSs are coordinate in a fair way as shown in Figure 10, it can be seen that as soon as the loading passes the critical limit, ESSs 1, 2, 3 and 4 start to coordinate and reduce the loading to the allowable range. However, ESSs 5, 6, 7 and 8 cannot be coordinated due to the communication loss between ESSs 4 and 5. The ratio of contribution of each ESS is as follow:

$$
\begin{gathered}
\frac{P_{1}}{P_{1}^{\max .}}=\frac{P_{2}}{P_{2}^{\max .}}=\frac{P_{3}}{P_{3}^{\max .}}=\frac{P_{4}}{P_{4}^{\max .}}=0.251 \\
\frac{P_{5}}{P_{5}^{\text {max. }}}=\frac{P_{6}}{P_{6}^{\max .}}=\frac{P_{7}}{P_{7}^{\max .}}=\frac{P_{8}}{P_{8}^{\max .}}=0
\end{gathered}
$$

As soon as the communication link is available at $t=600 \mathrm{~s}$, ESSs 5, 6, 7 and 8 will start to contribute to loading management and their contribution converge to 0.251 . So, comparing with the case with no communication drop, it can be seen that the communication drop causes this approach to use more resources than required (contributing ratio of 0.251 instead of 0.158 ). However, the robustness of the approach to keep the loading within the allowable range has still been achieved. 

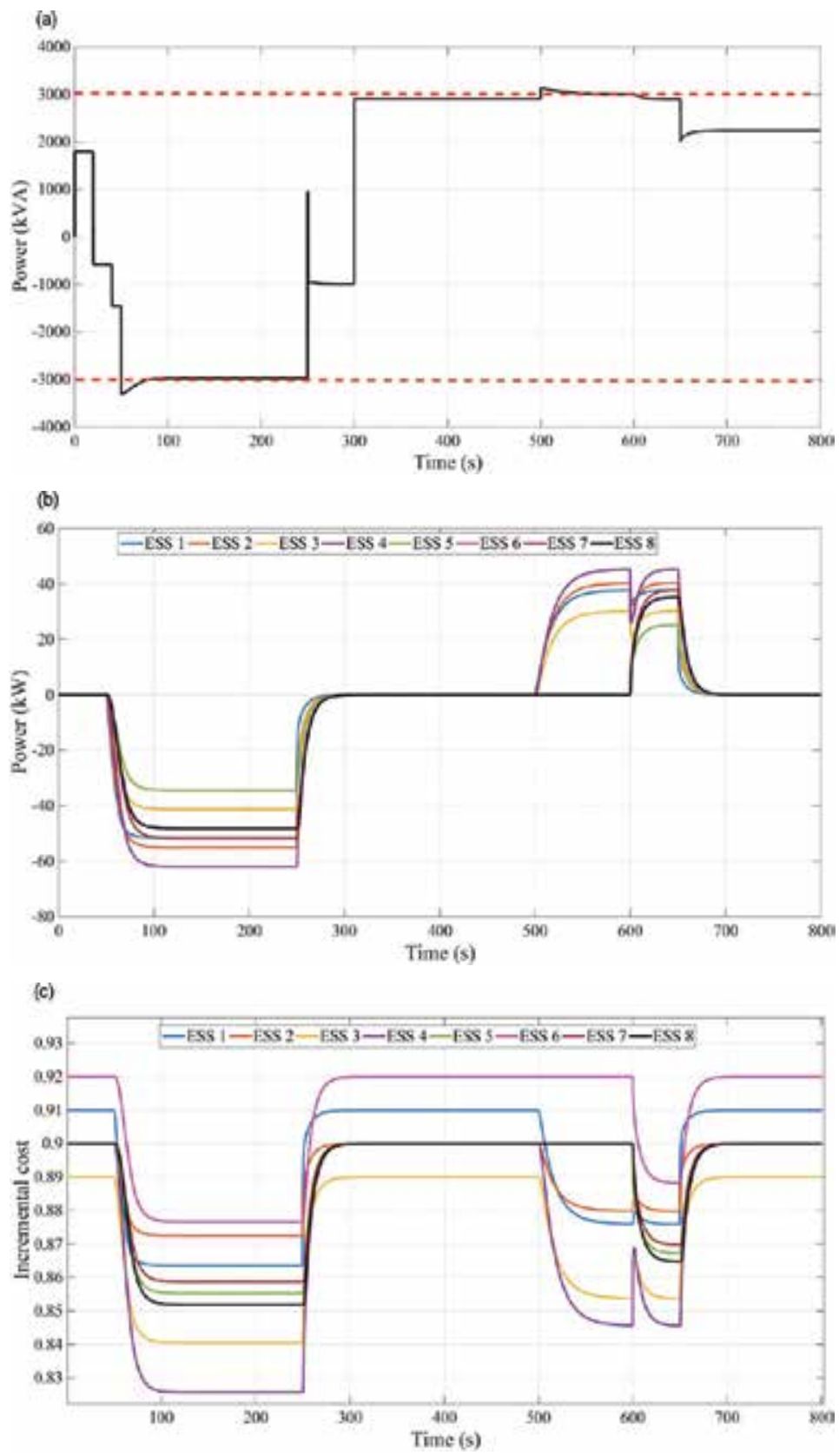

Figure 10. Impact of communication drop on fair sharing among ESSs (a) network loading, (b) ESS contribution, (c) incremental cost.

In the last case, the impact of communication drop was studied on the second approach. The same scenario is simulated for this case as well. The results are provided in Figure 11. Again, as there is no communication between ESS 4 and 5, only ESS 1, 2, 3 and 4 contributes in loading 
management. As soon as communication drop failure is repaired, ESSs 5, 6, 7 and 8 start to contribute in loading management. As the results show, all the ESSs have the same contribution compared with no communication drop case. Therefore, it can be said that communication drop may limit the resources while optimal operation is achieved all the time, in this case.
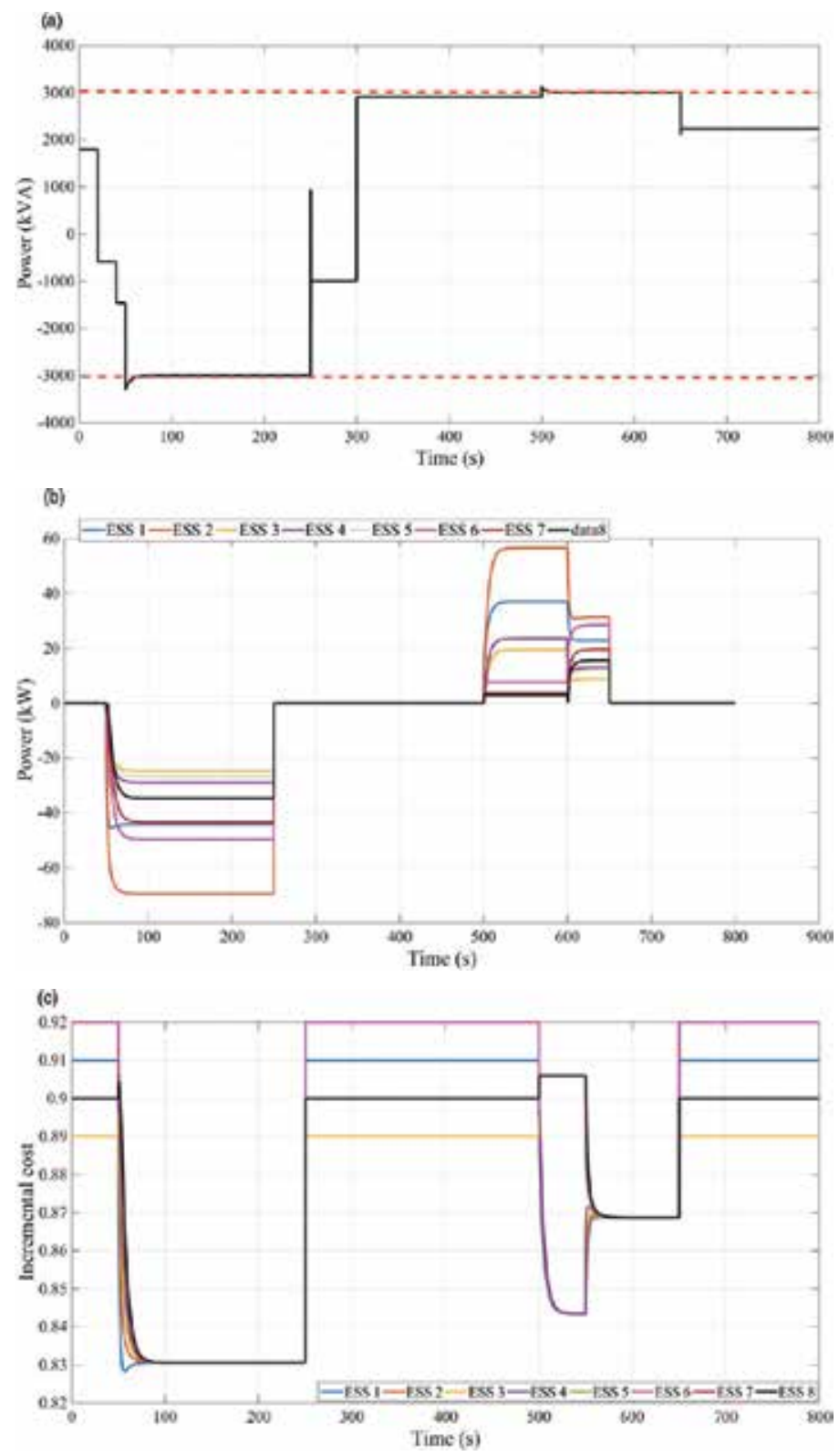

Figure 11. Impact of communication drop on optimal ESSs coordination, (a) network loading, (b) ESS contribution, (c) incremental cost. 


\section{Conclusions}

Future distribution networks with high utilisation of renewable energy resources can encounter network operating problems. Network voltage and loading issues are usually listed as the main network concerns which need to be addressed properly. As discussed and shown in this chapter, utilities and/or customers' storage resources can be used to resolve these issues. As part of this approach, distributed control method with consensus algorithm was presented in this book chapter. Application and results were also provided to support this technique over the existing methods, as a promising alternative to achieve accurate and efficient solution. Finally, the application of distributed control management strategy that coordinates multiple ESSs to deal with distribution network overloading was discussed in details. In this approach, both utility and customer interests were considered as part of a robust technique for loading management approach in future distribution network.

\section{Author details}

Ghassem Mokhtari ${ }^{1^{*}}$, Amjad Anvari-Moghaddam² and Ghavameddin Nourbakhsh ${ }^{3}$

*Address all correspondence to: ghassem.mokhtari@csiro.au

1 CSIRO, Brisbane, Australia

2 Department of Energy Technology, Aalborg University, Aalborg, Denmark

3 Queensland University of Technology, Brisbane, Australia

\section{References}

[1] A. Aquino-Lugo, R. Klump, and T. J. Overbye, "A control framework for the smart grid for voltage support using agent-based technologies," IEEE Trans. Smart Grid, vol. 2, no. 1, pp. 173-180, 2011.

[2] G. Mokhtari, G. Nourbakhsh, and A. Ghosh, "Smart coordination of energy storage units (ESUs) for voltage and loading management in distribution networks," IEEE Trans. Power Syst., vol. 28, no. 4, pp. 4812-4820, 2013.

[3] J. Ma and H. Yang, “Distributed parallel coordinated control strategy for provincialregional grid based on subarea division of the power system," in International Conference on Electricity Distribution (CICED), 2010 China. 2010, pp. 1-6.

[4] A. D. Dominguez-Garcia and C. N. Hadjicostis, "Coordination and control of distributed energy resources for provision of ancillary services," in IEEE International 
Conference on Smart Grid Communications (SmartGridComm), 2010 First. 2010, pp. 537542.

[5] K. Sun, L. Zhang, Y. Xing, and J. M. Guerrero, “A distributed control strategy based on DC bus signaling for modular photovoltaic generation systems with battery energy storage," IEEE Trans. Power Electron., vol. 26, no. 10, pp. 3032-3045, 2011.

[6] S. T. Cady, A. D. Dominguez-Garcfa, and C. N. Hadjicostis, "Robust implementation of distributed algorithms for control of distributed energy resources," in North American Power Symposium (NAPS). 2011, pp. 1-5.

[7] G. Mokhtari, A. Ghosh, G. Nourbakhsh, and G. Ledwich, "Smart robust resources control in lv network to deal with voltage rise issue," IEEE Trans. Sustain. Energy, vol. 4, no. 4, pp. 1043-1050, 2013.

[8] Y. Xu, W. Zhang, G. Hug, S. Kar, and Z. Li, “Cooperative control of distributed energy storage systems in a microgrid," IEEE Trans. Smart Grid, vol. 6, no. 1, pp. 238-248, 2015.

[9] R. Tonkoski, L. A. C. Lopes, and T. H. M. El-Fouly, "Coordinated active power curtailment of grid connected PV inverters for overvoltage prevention," IEEE Trans. Sustain. Energy, vol. 2, no. 2, pp. 139-147, 2011.

[10] P. Pillay and M. Manyage, "Definitions of voltage unbalance," IEEE Power Eng. Rev., vol. 21, no. 5, pp. 50-51, 2001.

[11] F. Shahnia, R. Majumder, A. Ghosh, G. Ledwich, and F. Zare, “Voltage imbalance analysis in residential low voltage distribution networks with rooftop PVs," Electr. Power Syst. Res., vol. 81, no. 9, pp. 1805-1814, 2011.

[12] R. Passey, T. Spooner, I. MacGill, M. Watt, and K. Syngellakis, “The potential impacts of grid-connected distributed generation and how to address them: a review of technical and non-technical factors," Energy Policy, vol. 39, no. 10, pp. 6280-6290, 2011.

[13] Y. Wang, P. Zhang, W. Li, W. Xiao, and A. Abdollahi, "Online overvoltage prevention control of photovoltaic generators in microgrids," IEEE Trans. Smart Grid, vol. 3, no. 4, pp. 2071-2078, 2012.

[14] G. Mokhtari, G. Nourbakhsh, F. Zare, and A. Ghosh, "Overvoltage prevention in LV smart grid using customer resources coordination," Energy Build., vol. 61, pp. 387-395, 2013.

[15] G. Mokhtari, G. Nourbakhsh, G. Ledwich, and A. Ghosh, “Overvoltage and overloading prevention using coordinated PV inverters in distribution network," in IECON 2014 -40th Annual Conference of the IEEE Industrial Electronics Society, 2014, pp. 5571-5574.

[16] E. Demirok, P. Casado González, K. H. B. Frederiksen, D. Sera, P. Rodriguez, and R. Teodorescu, "Local reactive power control methods for overvoltage prevention of distributed solar inverters in low-voltage grids," IEEE J. Photovolt., vol. 1, no. 2, pp. 174182, 2011. 
[17] Z. Akhtar, B. Chaudhuri, and S. Y. R. Hui, "Smart Loads for Voltage Control in Distribution Networks." IEEE Trans. Smart Grid, vol. 2, no.99, pp.1-10, 2016.

[18] B. P. Roberts and C. Sandberg, "The role of energy storage in development of smart grids," Proc. IEEE, vol. 99, no. 6, pp. 1139-1144, 2011.

[19] J. Hu, Y. Li, T. Yong, J. Cao, J. Yu, and W. Mao, “Distributed Cooperative Regulation for Multiagent Systems and Its Applications to Power Systems: A Survey," Sci. World J., vol. 2014, pp. 1-12, 2014.

[20] M. Yazdanian and A. Mehrizi-Sani, "Distributed control techniques in microgrids," IEEE Trans. Smart Grid, vol. 5, no. 6, pp. 2901-2909, 2014.

[21] W. Liu, W. Gu, Y. Xu, S. Xue, M. Chen, B. Zhao, and M. Fan, "Improved average consensus algorithm based distributed cost optimization for loading shedding of autonomous microgrids," Int. J. Electr. Power Energy Syst., vol. 73, pp. 89-96, 2015.

[22] Y. Xu and Z. Li, "Distributed optimal resource management based on the consensus algorithm in a microgrid," IEEE Trans. Ind. Electron., vol. 62, no. 4, pp. 2584-2592, 2015.

[23] P. Zhang, W. Li, and S. Wang, "Reliability-oriented distribution network reconfiguration considering uncertainties of data by interval analysis," Int. J. Electr. Power Energy Syst., vol. 34, no. 1, pp. 138-144, 2012. 

Chapter 2

\title{
Coordinated Demand Response and Distributed Generation Management in Residential Smart Microgrids
}

\author{
Amjad Anvari-Moghaddam, Ghassem Mokhtari and \\ Josep M. Guerrero
}

Additional information is available at the end of the chapter

http://dx.doi.org/10.5772/63379

\begin{abstract}
Nowadays with the emerging of small-scale integrated energy systems (IESs) in form of residential smart microgrids (SMGs), a large portion of energy can be saved through coordinated scheduling of smart household devices and management of distributed energy resources (DERs). There are significant potentials to increase the functionality of a typical demand-side management (DSM) strategy, and typical implementation of building-level DERs by integrating them into a cohesive, networked package that fully utilizes smart energy-efficient end-use devices, advanced building control/automation systems, and an integrated communications architecture to efficiently manage energy and comfort at the end-use location. By the aid of such technologies, residential consumers have also the capability to mitigate their energy costs and satisfy their own requirements paying less attention to the configuration of the energy supply system. Regarding these points, this chapter initially defines an efficient framework for coordinated DSM and DERs management in an integrated building and SMG system. Then a working energy management system (EMS) for applications in residential IESs is described and mathematically modeled. Finally, the effectiveness and applicability of the proposed model is tested and validated in different operating modes compared to the existing models. The findings of this chapter show that by the use of an expert EMS that coordinates supply and demand sides simultaneously, it is very possible not only to reduce energy costs of a residential IES, but also to provide comfortable lifestyle for occupants.
\end{abstract}

Keywords: demand response, distributed energy resources, energy management, smart microgrids, multi-objective optimization 


\section{Introduction}

The present intelligent energy networks together with the future smart electricity grids could highly affect the process of energy generation, transmission, distribution, and consumption at different levels (e.g., industrial, commercial, and residential sectors) in an efficient, reliable, and secure manner. By the use of these emerging technologies and advanced components in micro/ macro scales, not only the energy can be delivered to householders and business owners more cost-efficient, but also more renewable energy sources (RESs) can be exploited in a greener way. Through the joint operation of smart energy management systems (SEMSs) and advanced components within a smart microgrid (SMG) environment, it is possible to enable two-way digital communications between the utility and household devices and to provide the users with appropriate tools to improve their energy efficiency, consumption behavior and comfort levels [1]. On the other hand, optimal design, control and operation management of energyrelated production and consumption units have been attracting an increasing interest over the past decade and turning into a major part of energy management programs. Although the term "Energy Management" is widely used nowadays in literature and interpreted differently based on different operating scenarios, this chapter covers the subject of monitoring, controlling and conserving energy in building units and in residential SMGs, in broader perspective. In these environments, the users not only produce energy from several local and distributed generation (DG) units and play a "prosumer" role, but also participate in different incentive-based demand response (DR) programs to change their consumption behavior during different times of a day. However, integration of end-users as active components of future SMGs can inject unwanted risks and uncertainties in planning and operation phases [2,3]. To address these issues suitably, this chapter first outlines an efficient framework for coordinated DR and DG management in an integrated building and SMG system. Then, a fully-featured SEMS for managing loads at demand-side and domestic controllable generation units at the supply-side is described, mathematically modeled and validated.

\section{Smart microgrids (SMGs)}

In many parts of the world, the existing electricity networks at transmission and distribution levels use technologies, digital communication, control systems, and strategies that are many decades old. To update this aging infrastructure and to create an energy network that meets the ever-growing needs of today's power market, developed societies are aiming at creating intelligent facilities so as to use advanced sensing, communication, and control technologies to generate and distribute energy in a more efficient, economic, and secure fashion. Moreover, pursuing other objectives such as potential lower cost, higher service reliability, better power quality, increased energy efficiency and energy independence, is becoming the driving force to advocate the utilization of distributed energy resources (DERs) and to focus on what is called "Smart Microgrid", as the future of power systems. While details about the characteristics of a SMG vary greatly, key features include [4]: 


\section{- Reliable and resilient}

By the use of advance technologies such as smart metering, sensing, and state estimation, SMG improves fault detection and allows self-healing of the network without the intervention of technicians. This will ensure more reliable supply of electricity, and reduced vulnerability to natural disasters or attacks. SMG can also help utilities to speed outage restoration following major events, reduce the total number of affected customers, and improve overall service reliability to reduce customer losses from power disruptions [5].

\section{- Efficient and sustainable}

By the emergence and deployment of SMGs, efficiency improvement of energy infrastructures is expected. More than half of potential reductions in greenhouse gas (GHG) emissions would be achieved, transmission losses would be reduced, peak-load would be managed and transparency in electricity prices would be increased. By having a better understanding of equipment conditions through real-time equipment monitoring, utilities could also keep vital components operating at high efficiency. Moreover, through integration of digital technologies to the modernization of many sectors of the economy, higher efficiency gains, new opportunities, and greater productivity can be also guaranteed.

On the other hand, with the increasingly serious energy shortage and global warming, sustainable development can be obtained via integration of smart grid technologies, sustainable energy resources and low carbon emissions in power systems. The improved flexibility of the smart grid permits high penetration of green and sustainable RESs such as solar power and wind power, even without the addition of energy storage. However, the difficulties in dealing with intermittent power and the low utilization efficiency of power system appeared to be obstacles.

\section{- Flexible and bidirectional}

Figure 1 illustrates the way how the future smart electricity grids are different from the ones we know today. Our conventional energy networks have been designed and controlled in a way to support unidirectional flow of electricity and information from centralized large power generation units toward the end-use passive consumers, while the SMG relies more on bidirectional communication between consumers, suppliers and smart devices. The flow of energy across the network is also based on a mesh-grid structure rather than a unidirectional top-down system [6].

\section{- Demand-side management support}

SMG technology will allow customers to make more informed decisions about their energy consumption, adjusting both the timing and quantity of their electricity use. Such an ability which is called demand-side management (DSM) allows supply and demand sides to interact in an automated way in real-time, coordinating demand to flatten spikes. By doing this, the cost of adding reserve capacity is mitigated, wear and tear costs are reduced, and the life of equipment is increased. In a like manner, participating in DSM programs allows users to cut their energy bills by telling low priority devices to use energy only when it is cheapest. It should be noted that DSM programs comprise two different activities, demand 
response (DR) and energy efficiency/conservation (EE/C). A DR action transfers customer load during periods of high demand to off-peak periods and can reduce critical peak demand while $\mathrm{EE} / \mathrm{C}$ program encourages customers to give up some energy use in return for saving money and allows them to use less energy while receiving the same level of end service [7].

\section{- Market-enabling}

By the use of two-way communications between the suppliers/retailers and consumers it is possible to introduce more flexibility in operational strategies and enable effective market environments for suppliers who want to sell energy at higher prices and consumers who are willing to pay less. On the other hand, the development of market-driven operation procedures of the SMG will lead to a significant reduction of market power exerted by the established generation companies (GenCos). Widespread application of modular plug-andplay micro-sources may contribute to a reduction in energy price in the power market. Moreover, micro-sources may be used to provide ancillary services and further increase their market share in voltage support and stability services [8].

As a whole, SMG can be defined as an ingenious self-healing system that can be operated automatically by any source of fuel such as renewable energies and/or non-conventionals. It is an efficient way of RESs utilization and pollutant emission reduction. A SMG can realize existing overloads throughout the network and has the ability to reconfigure the network so as to impede potential outages. It is a base that enables active participation of und-users as informed consumers, accommodates all energy generation and storage options, advocates advanced products, markets or services, enables high penetration of intermittent sources, optimizes assets, resists attacks, and provides the energy quality for the range of needs in a digital economy $[6,9,10]$.

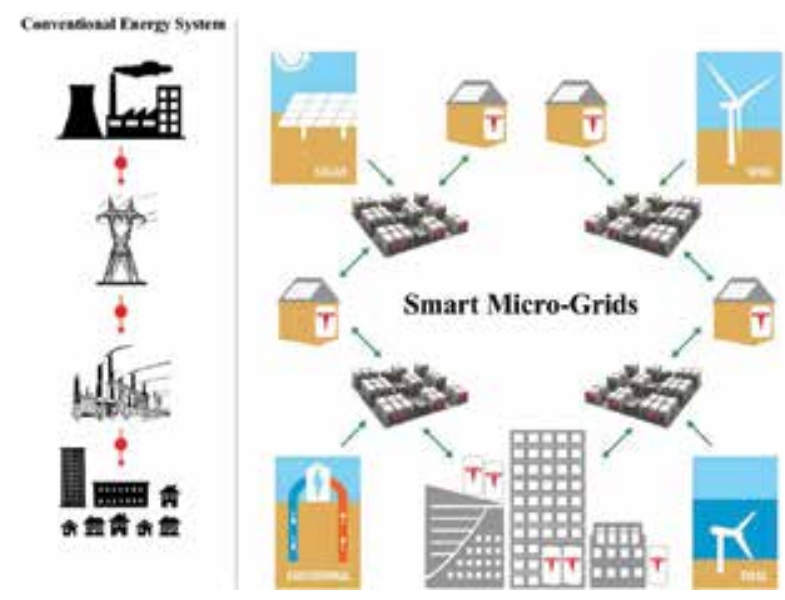

Figure 1. Conventional energy system vs. future smart micro-grids. 


\section{Integrated energy systems}

Integrated energy systems (IESs) provide the infrastructure to use SMGs to enable different mechanisms such as DR through SEMS. Smart energy management is an innovative approach to managing loads at the demand-side and domestic controllable units at the supply-side. It incorporates the conventional energy use management principles represented in DSM, DR, and DER programs and merges them in an integrated framework that simultaneously addresses permanent energy savings, demand reductions, and temporary peak load mitigations. In the context of residential SMGs, this is accomplished through an integrated dynamic system comprised of intelligent end-use devices (such as smart home appliances, lighting systems, and heating, ventilation, and air conditioning (HVAC)) and DERs with highly advanced sensing, controls and communications capabilities that enable real-time management of the system as a whole. A main residential SEMS consists of four components:

- Smart energy efficient end-use devices

- Smart DERs

- Advanced building control/automation systems

- Integrated communications architecture

The aforementioned components are built upon each other and interact with one another to provide an infrastructure that is intelligent, highly energy-efficient, automated, reliable and robust. The result is a system of systems that is capable of working in unison to optimize overall operation based on consumer requirements, utility constraints, available incentives and other variables such as weather and building occupancy. In the following section, the predominant characteristics of each of these four components are summarized.

\subsection{Smart energy efficient end-use devices}

The availability of system-wide electricity generation and transmission capacity can be increased for other uses through an investment in end-use energy efficiency. End-use energy efficiency, often referred to as a "negawatt", can be noted as a resource available to remove the mismatch between energy supply and demand, just as is done with other resources such as non-conventional or renewable power generations. Similar to other resources, saved energy from end-use efficiency is available in different amounts and levels of investment. When considering costs over the lifetime of an investment, end-use energy efficiency can be one of the lowest-cost means of meeting energy demand and of reducing GHG emissions [10]. On the other hand, to increase energy efficiency and reduce GHG emissions from the residential sector, there exist a number of solutions from which utilizing smart energy-efficient end-use devices is seemed to be a wise solution. Generally these devices includes but not limited to: efficient personal computers and peripherals (e.g., printers, scanners, and speakers), television and other audio-visual equipment, personal care appliances (e.g., hair dryers and electric toothbrushes), kitchen appliances (e.g., coffee makers, toasters, and microwaves), refrigeration and freezers, dishwashers, clothes washers and dryers, lighting, space conditioning, and 
integrated HVAC-water heating systems with the highest energy efficiencies. It is noteworthy that the smart end-use devices should be equipped with embedded features allowing for twoway communications and automated control.

\subsection{Smart DERs}

Over the past few years, the utility industry has made significant progress in defining common grid-supportive functions for distributed resources such as photovoltaics (PV), diesel engines, micro-turbines, and fuel cells (FCs), and also in defining the open standard communication protocols needed to make them smart and to connect these devices into SMGs. The functions include, for example [11,12]:

- Intelligent Volt-Var control

- Intelligent Volt-Watt control

- Reactive power/power factor

- Low-voltage ride through

- Load and generation following

- Storage systems charge/discharge management

- Connect/disconnect

- Dynamic reactive current injection (responding to changes in voltage $\mathrm{dV} / \mathrm{dt}$ )

- Maximum generation limiting

- Intelligent frequency-Watt control

- Peak limiting function for remote points of reference

\subsection{Advanced building control/automation systems}

Advanced building control/automation system is the key component of the future smart buildings that benefits from several communication domains, including the smart meter domain Advanced metering infrastructure (AMI), the internet domain and building area network (BAN). It is a system that receives information about task operating status, usage requests and network signals and sends control actions back to the smart devices, i.e., it optimizes the performance of end-use devices and DERs based on operational requirements, user preferences and external signals from the utility, end-user or other authorized entity. Such a system also provides the occupants with useful feedbacks about energy usage pattern, and helps making control decisions more autonomously. To identify solutions based on different objectives (e.g., energy saving and living comfortably), it also gathers information from the home's environment as well as the outside situation [13].

Other controllers that allow for two-way communications and those that have the ability to learn from past experience and apply that knowledge to future events are also playing crucial role in an advanced building control systems. 


\subsection{Integrated communications architecture}

A typical integrated communication architecture allows automated control of smart end-use devices and DERs in response to various signals such as pricing or load reduction signals from the utility, weather forecast in an hourly/daily basis, and bidirectional data transmission (such as external alerts as well as end-user signals) between multiple nodes. It also allows the enduse devices, DERs and/or control systems to send operational data to external parties (e.g., advanced meters that communicate directly with utilities).

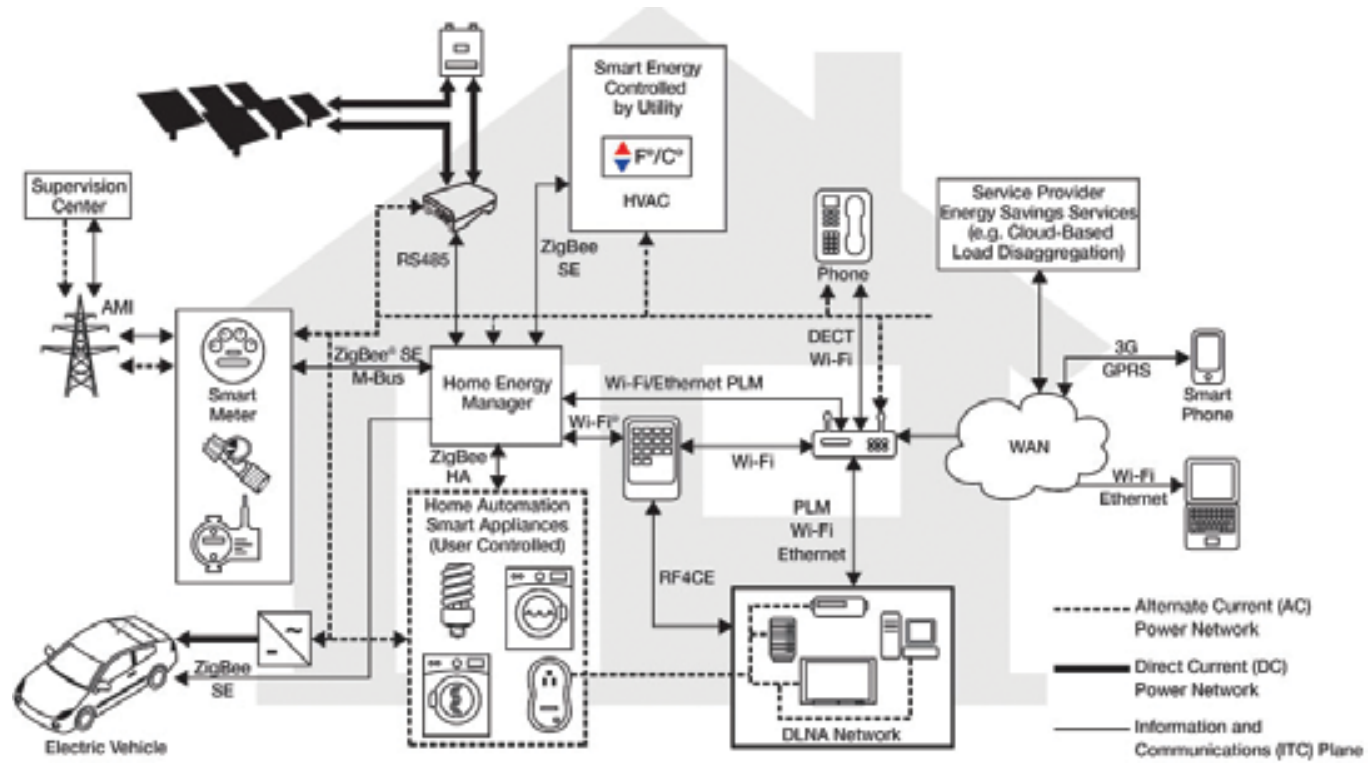

Figure 2. Energy management infrastructure for residential buildings.

Figure 2 shows an example of an energy management infrastructure applied to a generic residential building. As can be seen, building energy management systems (EMSs), and to a broader extent BAN, are not single-technology networks, but combine various specialized networking technologies. Interoperability and coexistence are key to guaranteeing the cooperation between all protocols in the same area, especially when building EMSs need to coexist with legacy home automation, home security systems or home A/V systems. In this example, there are two-way communications via the Internet, Ethernet PLM, as well as via the power line or ZigBee. The building is equipped with smart energy-efficient end-use devices, an energy manager, automated controls with data management capabilities, DERs such as rooftop solar PVs, and other on-site generation and storage systems such as electric vehicles (EVs). Thus, energy-efficient devices, controls and DR strategies are coupled with on-site energy sources to serve as an additional energy "resource" for the utility. Not only do all of these elements contribute to the utility's supply-side by reducing building demand, DERs can 
also feed excess power back to the grid. A SEMS is likely to have a much larger impact on a building's electricity consumption and demand than just implementing energy efficiency and/ or DR on their own.

\section{SEMS operation from an integrated perspective}

As described earlier, smart end-use devices which benefit from advanced highly efficient controls, sensing and communications capabilities are regarded as key components of a SEMS. These devices can dynamically communicate with other smart components and adjust their performance in response to external reference signals. This marks an emergence from static to dynamic end-use devices with advancements in distributed intelligence. In addition to electric end-use devices, a SEMS would also include DERs such as solar PV systems, wind turbines (WTs), micro-combined heat and power (micro-CHP) units, diesel generators, and FCs. The performances of these DERs are also programmed to operate in an integrated manner with end-use devices at the facility so as to be able to optimize overall system performance based on the predefined goals and objectives. Here, we use "smart device" as a common term referring either to an energy-efficient end-use device or to a controllable DER. Each programmable smart device has its own control strategy, which assures optimal performance of the device based on external reference signals coming from SEMS and a variety of external parameters such as weather conditions, energy price signals, consumer habits and user's preferences. For interoperability among the smart devices and other components within a SEMS's domain, advanced meters with two-way communications infrastructure are also required. This will enable the SEMS to connect the electric meter and smart devices in the building to the BAN, thereby giving the SEMS direct access and control of these devices. Depending on the hourly energy price or other external parameters and based on the predefined objectives and available constraints, the smart devices equipped with the responsive controls automatically respond to the external signals and optimize entire system performance, say within the user "comfort range" to minimize energy costs. For autonomous operation in response to environmental conditions and other influential parameters within the controlled space, SEMS must be capable of very abstract decision making, ranging from determining a meaningful balance between cost and comfort for current conditions, to the very physical, such as turning a smart device on or off. Within a building unit, the response strategy of each smart device is also networked and interacts with the response strategies of other devices in a way to optimize the entire system performance. The system should be also able to execute a fully automated control strategy with override provisions, i.e., although the system is able to control multiple devices automatically, user preference may be dominated to autonomous operation and direct control is adopted accordingly. Likewise, for devices that are controlled indirectly, signaling approaches with some means of indication (such as blinking lights, colored LEDs) can be applied for the occupants' awareness to assist them when is propitious to run these appliances. For a SEMS to be able to interact effectively with its environment and learn from prior experiences without being explicitly programmed, learning functionalities with learning logic and artificial intelligence can be also integrated into the system. In this way, the system 
searches through data to look for patterns. However, instead of extracting data for human comprehension, machine learning is used to process data, improve the program's own understanding and adjust program actions accordingly.

\section{SEMS modeling and design for IESs}

Energy management for an IES includes optimal scheduling and running of different energyrelated generation devices as well as consumption units considering predefined goals such as energy conservation, environment protection and cost savings. It is also connected tightly to the people's way of life and their comfort zones. In this regard, a residential energy management (REM) strategy can be seen as a multiple-criteria optimization and decision-making problem that should be handled in a way to meet the system's goals and constraints. For this problem, it is crucial to model the components of the IES carefully. In the following subsections different compartments of a REM problem are introduced and mathematically modeled.

\subsection{Heat transfer and thermal modeling of a residential building unit}

For diagnosis and control strategy analysis, there is a strong need to develop suitable thermal model for different components of a residential building unit. Based on a simplified lumped capacitance model, the thermal resistance across a layer of area $(A)$, thickness $(x)$, and thermal conductivity $(k)$ is as follows [2]:

$$
R_{\text {layer }}=\frac{x}{k \cdot A}=\frac{R_{\text {value }}}{A}
$$

where $R_{\text {value }}$ denotes the insulation level of the layer. As an example, for a multi-layer exterior wall such as one depicted in Figure 3, the total thermal resistance can be calculated as follows:

$$
\begin{gathered}
R_{T}=\frac{\sum_{i=1}^{3} R_{T i}}{2} \\
R_{T i}=\frac{100}{\frac{\text { \%areawith frame }}{R I_{F, i}}+\frac{\% \text { area without frame }}{R I_{I, i}}}
\end{gathered}
$$

where $R T_{1}$ and $R I_{F, i}$ are the thermal resistances in insulation and framed wall, respectively. $R I_{I, i}$ is the thermal resistance in insulated portion. $R T_{2}$ is the thermal resistance between the planes bounding the inner and outer faces of the metal framing members, and $R T_{3}$ is the resistance of remaining components. Likewise, $R_{T}$ is the total thermal resistance. 


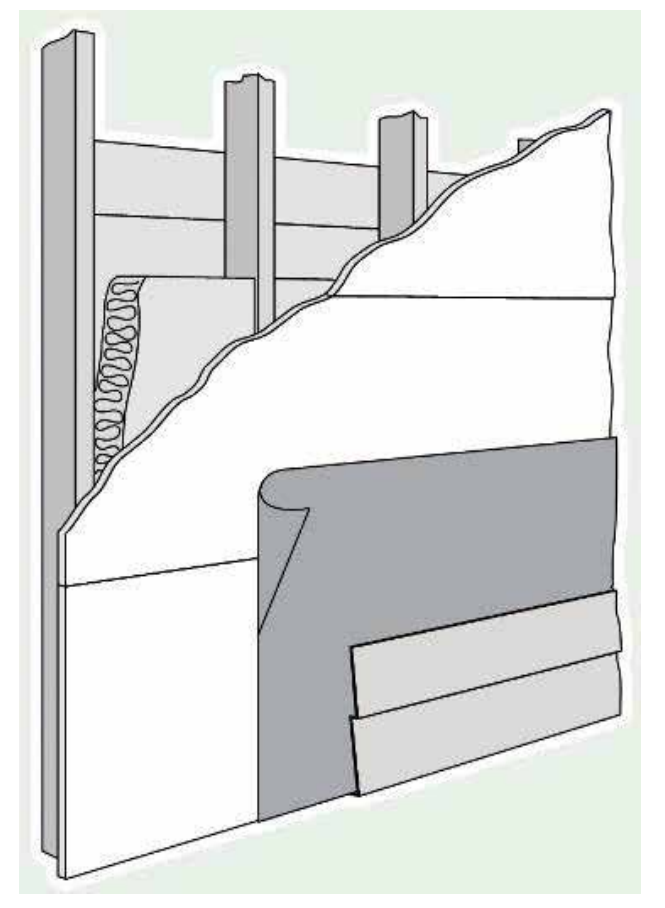

Figure 3. A typical steel stud framing wall insulating sheathing [2].

Having calculated thermal resistances for different materials and components of a house structure using the same procedure, one can easily evaluate the amount of heat flows between different nodes as follows:

$$
\begin{gathered}
\phi_{i o}(h)=\frac{T_{\text {in }}(h)-T_{\text {out }}(h)}{R_{i o}} \\
\phi_{f i}(h)=\frac{T_{f}(h)-T_{\text {in }}(h)}{R_{f i}} \\
\phi_{f g}(h)=\frac{T_{f}(h)-T_{g}(h)}{R_{f g}}
\end{gathered}
$$

in which, $\varphi_{i o}$, is the heat flow between the indoor air node and the outdoor environment through thermal resistance $R_{i o r} \varphi_{f i}$ is the heat flow between the floor and the indoor air through thermal resistance $R_{f i}$ and $\varphi_{f g}$ is the heat flow between the floor and the ground through thermal resistance $R_{f g}$ as shown in Figure 4 . In a similar manner, $T_{\text {in }}(h), T_{\text {out }}(h), T_{f}(h)$, and $T_{g}(h)$ are the temperatures of the indoor air, the outdoor environment, the floor, and the ground at hour $h$. 


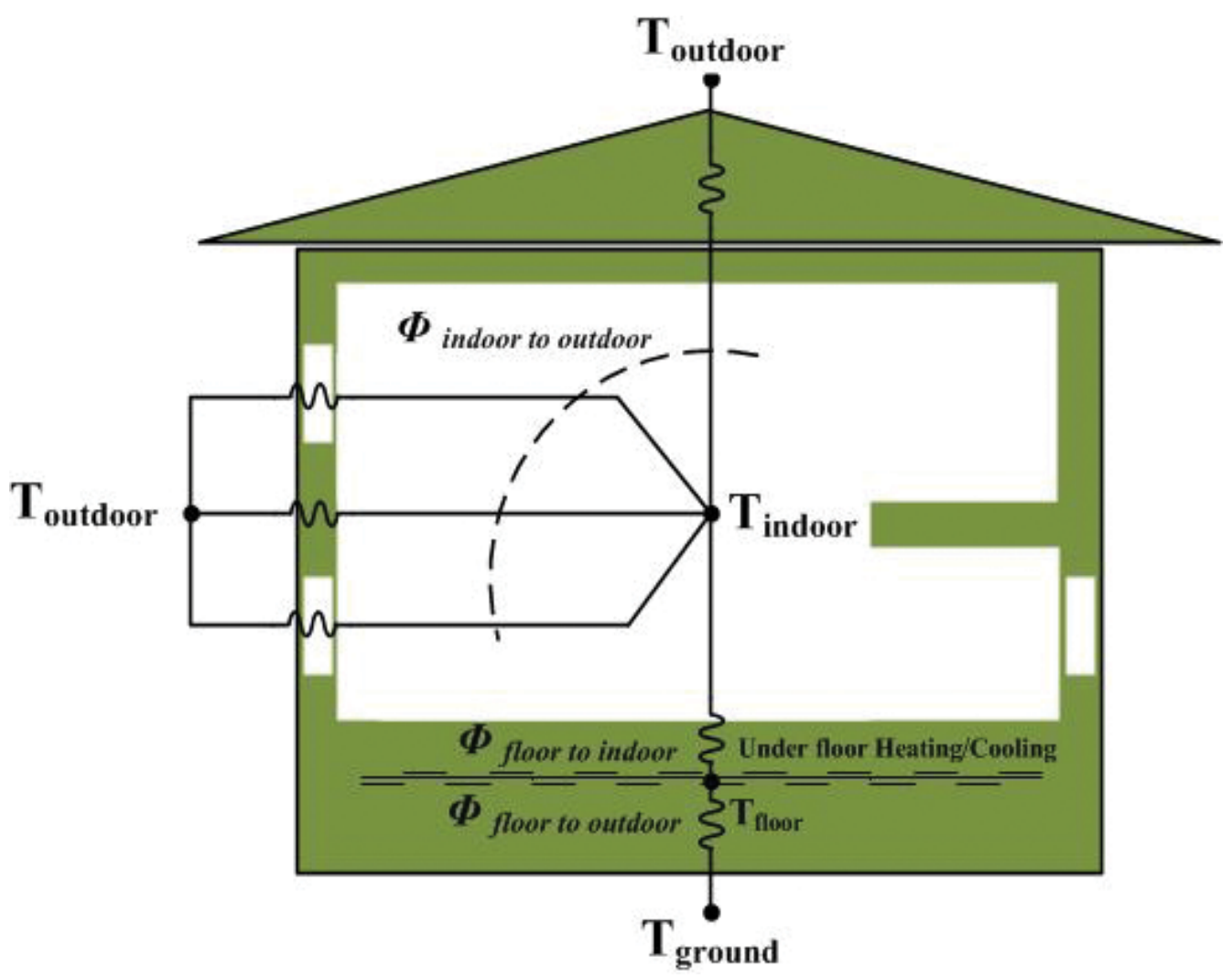

Figure 4. Thermal modeling of a building.

Regarding to an under-floor heating/cooling system (RFH/CS) as a heat node in the house, the amount of thermal energy that is supplied to the floor is determined as follows:

$$
\phi_{H P}(h)=\left(u_{H P}(h) \cdot \eta_{H}(h)-\left(1-u_{H P}(h)\right) \cdot \eta_{C}(h)\right) P_{H P}(h)
$$

where $u_{H P}$ is a binary variable stands for heating ("1") or cooling ("0") status, and $P_{H P}\left[0, P_{H P, \max }\right]$ is the power consumption of the heat pump at hour $h . \eta_{H}\left[\eta_{H, \min }, \eta_{H, \max }\right]$ and $\eta_{C}\left[\eta_{C, \min ,}, \eta_{C, \max }\right]$ are the heating and cooling coefficients of performance (COPs) which are roughly linear functions of outdoor temperature.

As another source of thermal energy, solar radiation has a great effect on the heating/cooling load of a building. During different months in a year, the Sun's path varies across the sky and affects the overall thermal behavior of the building by its direct and diffuse radiation [14]. As shown in Figure 5, the hourly heat flow into an exterior surface of a building due to solar radiation can be introduced as follows: 


$$
\begin{aligned}
& \phi_{\text {surf }}(h)=h_{o} A_{s}\left(T_{\text {out }}(h)-T_{\text {surf }}(h)\right)+\alpha_{s} A_{s} \varphi_{\text {solar }}(h)-\varepsilon A_{s} \sigma\left(T_{\text {out }}^{4}(h)-T_{\text {surr }}^{4}(h)\right) \\
& \quad=h_{o} A_{s}\left(T_{\text {eq_out }}(h)-T_{\text {surf }}(h)\right)
\end{aligned}
$$

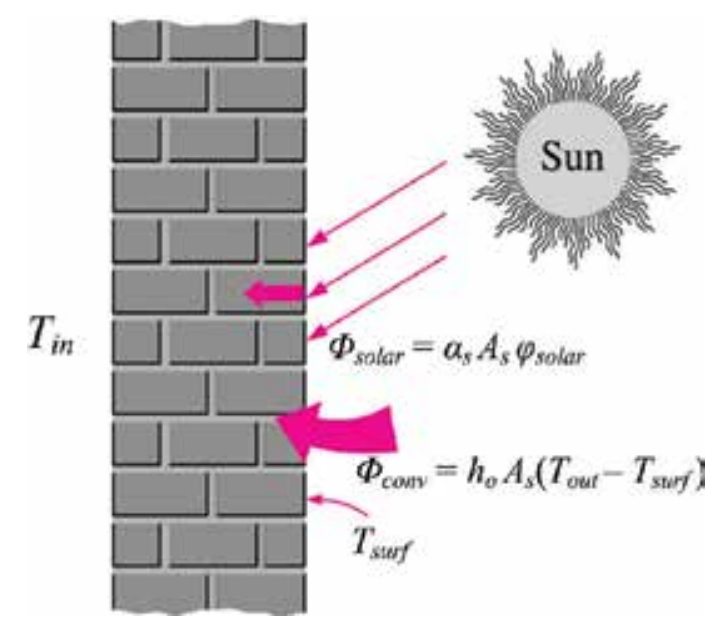

Figure 5. The solar radiation effect on heating/cooling load of a building.

where $h_{o}$ is the combined convection and radiation heat transfer coefficient, $\alpha_{s}$ is the solar absorptivity, $\varepsilon$ is the emissivity of the surface, $\phi_{\text {solar }}$ is the solar radiation incident on the surface and $\sigma$ is Stefan-Boltzmann constant. $T_{\text {surf }}$ and $T_{\text {surr }}$ are the average temperatures of the exposed surfaces and other surrounding surfaces, respectively. Likewise, $T_{\text {eq_out }}$ is the equivalent temperature of outdoor air considering the effect of solar radiation. The previous equation can be rewritten as:

$$
T_{\text {eq_out }}(h)=T_{\text {out }}(h)+\frac{\alpha_{s} \varphi_{\text {solar }}(h)}{h_{o}}-\frac{\varepsilon \sigma\left(T_{\text {out }}^{4}(h)-T_{\text {surr }}^{4}(h)\right)}{h_{o}}
$$

Once $T_{\text {eq } \_ \text {out }}$ is available, heat transfer through an exterior surface with the overall heat transfer coefficient of $U$, thermal resistivity of $R_{s i}$ and surface area of $A_{\mathrm{s}}$ into the indoor environment can be expressed as:

$$
\phi_{s i}(h)=U A_{s}\left(T_{\text {eq_out }}(h)-T_{\text {in }}(h)\right)=\frac{T_{\text {eq_out }}(h)-T_{\text {in }}(h)}{R_{\text {si }}}
$$

The internal heat gain of a building unit is also affected by a number of factors such as the heat generated by the occupants (i.e., occupant metabolisms), lights and appliances (e.g., stove, 
television, and radio). Although this heat gain cannot be determined exactly, its average amount can be estimated from the people's lifestyle. As an example, Table 1 shows the metabolic rates per unit body surface area for various activities [15]:

$$
A_{b o d y}=0.202 \times m^{0.425} \cdot L^{0.725}
$$

\begin{tabular}{|c|c|c|c|c|c|}
\hline$\overline{\text { Activity }}$ & & $\begin{array}{l}\text { Metabolic } \\
\text { rates } \\
\left(\mathrm{w} / \mathrm{m}^{2}\right)\end{array}$ & Activity & & $\begin{array}{l}\text { Metabolic } \\
\text { rates } \\
\left(\mathrm{w} / \mathrm{m}^{2}\right)\end{array}$ \\
\hline \multirow[t]{4}{*}{ Resting } & Sleeping & 40 & Driving/flying & Car & 60-115 \\
\hline & Reclining & 45 & & Aircraft, routine & 70 \\
\hline & $\begin{array}{l}\text { Seated, } \\
\text { quiet }\end{array}$ & 60 & & Heavy vehicle & 185 \\
\hline & $\begin{array}{l}\text { Standing, } \\
\text { relaxed }\end{array}$ & 70 & $\begin{array}{l}\text { Miscellaneous } \\
\text { occupational }\end{array}$ & Cooking & 95-115 \\
\hline \multirow[t]{3}{*}{$\begin{array}{l}\text { Walking (on } \\
\text { the level) }\end{array}$} & $\begin{array}{l}2 \mathrm{mph} \\
(0.89 \mathrm{~m} / \mathrm{s})\end{array}$ & 115 & activities & Cleaning house & $115-140$ \\
\hline & $\begin{array}{l}3 \mathrm{mph} \\
(1.34 \mathrm{~m} / \mathrm{s})\end{array}$ & 150 & Machine work & Light & $115-140$ \\
\hline & $\begin{array}{l}4 \mathrm{mph} \\
(1.79 \mathrm{~m} / \mathrm{s})\end{array}$ & 220 & & Heavy & 235 \\
\hline \multirow[t]{7}{*}{$\begin{array}{l}\text { Office } \\
\text { activities }\end{array}$} & $\begin{array}{l}\text { Reading, } \\
\text { seated }\end{array}$ & 55 & & $\begin{array}{l}\text { Handling } 50-\mathrm{kg} \\
\text { bags }\end{array}$ & 235 \\
\hline & Writing & 60 & & $\begin{array}{l}\text { Pick and shovel } \\
\text { work }\end{array}$ & $235-280$ \\
\hline & Typing & 60 & $\begin{array}{l}\text { Miscellaneous } \\
\text { leisure }\end{array}$ & $\begin{array}{l}\text { Dancing, } \\
\text { social }\end{array}$ & $140-255$ \\
\hline & $\begin{array}{l}\text { Filing, } \\
\text { seated }\end{array}$ & 70 & activities & $\begin{array}{l}\text { Calisthenics/ } \\
\text { exercise }\end{array}$ & $175-235$ \\
\hline & $\begin{array}{l}\text { Filing, } \\
\text { standing }\end{array}$ & 80 & & Tennis, singles & $210-270$ \\
\hline & $\begin{array}{l}\text { Walking } \\
\text { about }\end{array}$ & 100 & & Basketball & $290-440$ \\
\hline & Lifting/packing & 120 & & $\begin{array}{l}\text { Wrestling, } \\
\text { competitive }\end{array}$ & $41-505$ \\
\hline
\end{tabular}

Table 1. Metabolic rates during various activities. 
where $m$ is the mass of the body in kilogram, and $L$ is the height in meter.

Considering all the heat flows described earlier, the thermal behavior of a building in terms of temperature update functions can be determined as $[2,12]$ :

$$
\begin{gathered}
T_{i n}(h)=T_{i n}(h-1)+\frac{\Delta h_{\text {step }}}{m_{i} c_{p, i}}\left(\phi_{f i}(h)+\phi_{s i}(h)+\phi_{b p}(h)-\phi_{i o}(h)\right) \\
T_{f}(h)=T_{f}(h-1)+\frac{\Delta h_{\text {step }}}{m_{f} c_{p, f}}\left(\phi_{H P}(h)+\phi_{s f}(h)-\phi_{f g}(h)-\phi_{f i}(h)\right)
\end{gathered}
$$

where $m_{f}\left(m_{i}\right)$ and $c_{p, f}\left(c_{p, i}\right)$ are the floor (indoor air) mass and specific heat capacity coefficients, respectively, and $\Delta h_{\text {step }}$ is the time step. Likewise, $\varphi_{b p}(h)$ is the house background power calculated by the hourly internal heat gain of the building. $\varphi_{s f}(h)$ denotes the heat obtained from direct solar radiation:

$$
\phi_{s f}(h)=\alpha_{f} \cdot A_{s f} \cdot \varphi_{\text {solar }}(h)
$$

where $\alpha_{f}$ and $A_{s f}$ are the solar absorptivity and the area of the floor on the sunny side.

\subsection{Schedulable tasks and residential load model}

To derive the electrical load model of a residential building unit, it is very important to understand the behaviors of different household appliances and devices. From controllability prospective, in-home appliances are normally categorized into Class I (i.e., non-schedulable) and Class II (i.e., schedulable) appliances. Class I devices which is also labeled as "manually operated" or "non-schedulable" tasks have their own fixed power consumption rates $\left(P_{D f i x}\right)$ and must be operated upon the user's request. From the other side, Class II appliances which are further sub-classified as "temperature-shiftable" and "time-shiftable" tasks have the capability to be controlled either automatically or manually [2]. HVAC and refrigerators are examples of "temperature-shiftable" devices that are normally running hour after and can be stopped once in a while provided that an acceptable temperature interval is guaranteed. Differently, a number of appliances such as washing machine, dishwasher, and dryer which are regarded as "time-shiftable" tasks can be operated at planned or desired time-intervals. For optimal operation of such devices, there exist several parameters that need to be set by residents [13]:

- utilization time range $\left(U T R_{i}=\left[h_{s, i} h_{f, i}\right]\right)$ during which, task $i$ is valid for scheduling,

- preferred time range $\left(P T R_{i}=\left[h_{e, i} h_{l, i}\right]\right)$ during which, task $i$ is better to be scheduled according to the user's preferences, 
- length of operation time $\left(L O T_{i}\right)$ during which the task operation is completed, and

- estimated energy consumption $\left(E E C_{i}\right)$.

Through these definitions, the power consumption of shiftable task $i$ at hour $h$ would be:

$$
P_{D s c h d, i}(h)=\frac{E E C_{i}}{L O T_{i}} \cdot s_{i}(h) ; \forall\left(h \in U T R_{i}, i \in N\right)
$$

where $s_{i}(h)$ is a binary variable showing the $i^{\text {th }}$ device status as "scheduled: $1^{\text {" }}$ or "dropped: 0 ". There are also several constraints that must be met suitably for each task $i \in N$ :

First, task $i$ must be completed before the end of optimization time $h_{f, i}$ :

$$
\sum_{h=h_{s, i}}^{h_{f, i}} s_{i}(h)=L O T_{i}
$$

Second, some tasks need to run once within a time window and should not be turned off before the completion:

$$
\sum_{h=h_{s, i}}^{h_{f, i}}\left|s_{i}(h)-s_{i}(h-1)\right| \leq 2
$$

Third, one task (e.g., task $j$ ) may depend on the completion of another task (e.g., task $i$ ):

$$
\sum_{h=h_{s, j}}^{h_{f, j}} s_{j}(h) \cdot H\left(\lambda-L O T_{i}+\sum_{h=h_{s}}^{h} s_{i}(\widehat{h})\right)=L O T_{j}
$$

where $h_{s}=\min \left(h_{s, i} h_{s, j}\right), 0<\lambda<1$, and $H()$ is the Heaviside step function. The following constraint must be also considered if a definite time gap between the operations of two consecutive tasks is desired:

$$
\begin{aligned}
& \operatorname{Ord}(\widehat{h}) \cdot H\left(s_{j}(\widehat{h})-s_{j}(\widehat{h}-1)-\lambda\right) \leq(\operatorname{Ord}(h)-1) \cdot H\left(s_{i}(h-1)-s_{i}(h)-\lambda\right) \\
& +\Lambda_{i, j} ; \forall\left(h \in U T R_{i}, \widehat{h} \in U T R_{j}\right)
\end{aligned}
$$

in which, $\Lambda_{i, j}$ denotes the largest allowed time gap and $\operatorname{Ord}()$ shows the time order in the examined period. Maximum power consumption of a building unit $\left(P_{\text {House }}^{\max }\right)$ must be also included as a technical constraint: 


$$
P_{D}(h)=P_{D f f i x}(h)+\sum_{i=1}^{N} P_{D s c h d, i}(h) \leq P_{\text {House }}^{\max }
$$

\subsection{Modeling of distributed generation and storage units}

In a typical IES there exist some means of energy generation and storage both in forms of nonconventionals and renewables. This section presents the mathematical modeling of low voltage (LV) grid-connected distributed generation (DG) units.

\subsubsection{Wind-powered electrical generators (wind turbines)}

Today's utility-scale LV grid-connected WTs are extensively utilized for grid-support applications as well as empowering local loads. The amount of power generated at a WT site depends on the wind speed $(V)$, air density at the location $(\rho)$, the turbine power rating and its technical specifications such as performance coefficient $\left(C_{p}\right)$ and generator and gearbox efficiencies $\left(N_{g}\right.$ and $\left.N_{b}\right)$. In this regard, the electrical power output of a wind-powered electrical generator can be described as [2]:

$$
P_{W T}=\frac{V^{3}(\rho \cdot A)\left(C_{p}\right)\left(N_{g} \cdot N_{b}\right)}{2}
$$

\subsubsection{PV power system}

Similar to other RESs, photovoltaic power systems (PVs) can be used for electrification of domestic demands. PVs are ranged from small-scale systems with power capacities of kilowatts (such as rooftop-mounted or building-integrated) to large utility-scale power plants with several megawatts capacity. The amount of electric power generated by a PV module is also depended on multiple factors including but not limited to, the array rated capacity $\left(Y_{P V}\right)$, system derating factor $\left(f_{P V}\right)$, cell temperature in real operating and standard test conditions $\left(T_{c}, T_{c, \text { STC }}\right)$, temperature coefficient of power $\left(\alpha_{p}\right)$ and solar radiation incident in real and standard test conditions $\left(G_{T}, G_{T, S T C}\right)[13]$ :

$$
P_{P V}=\left(\frac{G_{T}}{G_{T, S T C}}\right) \cdot\left(Y_{P V} \cdot f_{P V}\right) \cdot\left[1+\alpha_{p}\left(T_{c}-T_{c, S T C}\right)\right]
$$

\subsubsection{Micro-combined heat and power system (micro-CHP)}

FC based micro-CHP is another highly-efficient, low-maintenance means of cogeneration at residential places where quiet operation is also intended. Similar to other combined heat and power facilities, a FC-based micro-CHP system encompasses three subsystems including a hot 
water storage tank (DHW), a FC unit and an auxiliary boiler. As shown in Figure 6, a typical micro-CHP consumes natural gas $g_{\mathrm{CHP}}$ and converts it into the heat $\left(P^{\text {th }}{ }_{\mathrm{CHP}}\right)$ and electricity $\left(P_{C H P}^{e}\right)$ with corresponding efficiencies $\left(\eta_{e}, \eta_{t h}\right)$ considering electrical/thermal power limits and ramp-rates as follow [2]:

$$
\begin{gathered}
P_{C H P, \min }^{e} \leq P_{C H P}^{e}(h)=g_{C H P}(h) \cdot \eta_{e} \leq P_{C H P, \max }^{e} \\
P_{C H P, \min }^{t h} \leq P_{C H P}^{t h}(h)=g_{C H P}(h) \cdot \eta_{t h} \leq P_{C H P, \max }^{t h} \\
\left|P_{C H P}^{e}(h)-P_{C H P}^{e}(h-1)\right| \leq P_{C H P, \text { ramp }}^{e} \\
\left|P_{C H P}^{t h}(h)-P_{C H P}^{t h}(h-1)\right| \leq\left(\eta_{t h} / \eta_{e}\right) \cdot P_{C H P, \text { ramp }}^{e}
\end{gathered}
$$

To mathematically model the behavior of a hot water storage tank, the energy equivalent of the stored water should be considered as follow:

$$
Q_{s t}(h+1)=Q_{s t}(h)+\left(P_{C H P}^{t h}(h)+P_{a u x}^{t h}(h)-P_{D}^{t h}(h)-P_{\text {loss }}^{t h}(h)\right) \cdot \Delta h_{\text {step }}
$$

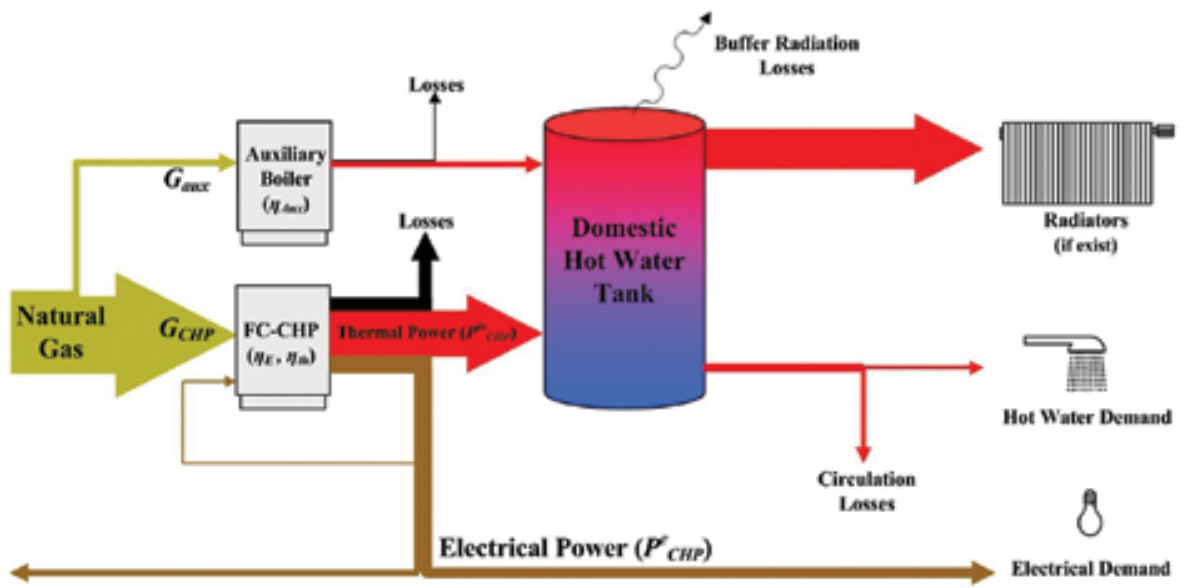

Figure 6. Energy flows in a FC-based co-generation system. 
in which $Q_{s t}(h)$ is the energy content of the storage at hour $h, P_{D}^{\text {th }}(\mathrm{h})$ and $P^{\text {th }}$ loss $(\mathrm{h})$ are the heat demand and heat losses at hour $h$, respectively. From the above equation, the temperature update function of the hot water at each time step can be derived as follow [2]:

$$
\begin{gathered}
T_{s t}(h+1)=\frac{V_{D}^{t h}(h) \cdot\left(T_{c w}-T_{s t}(h)\right)+V_{t o t} \cdot T_{s t}(h)}{V_{t o t}}+\frac{P_{C H P}^{t h}(h)+P_{a u x}^{t h}(h)}{V_{t o t} \cdot C_{w}} \\
-\left(\frac{A_{s t}}{V_{t o t} \cdot C_{w} \cdot R_{s t}}\right) \cdot\left(T_{s t}(h)-T_{b}(h)\right) \\
T_{s t \text {, } \min } \leq T_{s t}(h) \leq T_{s t, \max }
\end{gathered}
$$

where $T_{c w}$ and $T_{b}(h)$ are the entering cold water and environment temperatures at hour $h, V_{\text {tot }}$ and $V^{t h}$ are the total tank volume and hourly hot water demand (HWD) in liter, respectively. $A_{s t}$ denotes the area of the storage tank covered by a material with insulation level of $R_{s t}$.

\subsubsection{Energy storage system (ESS)}

ESSs are becoming an important part of today's smart grid applications where high penetration of renewable energies and reliable power generation is required. The behavior of an ESS which is labeled as battery in this chapter, can be presented based on an energy state update function as:

$$
\begin{gathered}
\operatorname{SOC}(h+1)=\operatorname{SOC}(h)+\frac{\left(P_{\text {Batt }}^{c h}(h)-P_{\text {Batt }}^{\text {dch }}(h)\right) \cdot \Delta h_{\text {setp }}}{E_{\text {Batt }}} \\
\operatorname{SOC}_{\text {min }} \leq \operatorname{SOC}(h) \leq S O C_{\text {max }}
\end{gathered}
$$

where $S O C(h)$ stands for the battery state of charge at hour $h, S O C_{\min }\left(S O C_{\max }\right)$ is the lower (upper) bound of battery's $S O C$, and $E_{\text {Batt }}$ is the battery capacity in $k W h$. Likewise, $P_{\text {Batt }}^{\text {and }}$ and $P_{\text {Batt }}^{d c h}$ are the charging and discharging power of the battery which are limited by the following constraints:

$$
\begin{gathered}
P_{\text {Batt }}^{c h}(h) \leq P_{\max }^{c h} \cdot \eta_{c h} \cdot u_{\text {Batt }}(h) \\
P_{\text {Batt }}^{d c h}(h) \leq\left(\frac{P_{\max }^{d c h}}{\eta_{\text {dch }}}\right) \cdot\left(1-u_{\text {Batt }}(h)\right)
\end{gathered}
$$


where $u_{\text {Batt }}$ is a binary variable denotes the operating status as charging " 1 " or discharging " 0 ".

\subsection{Objective functions}

As shown in Figure 7, flowchart diagram, a residential energy scheduling and management problem can be viewed as a multi-criteria decision-making model and an optimization problem with different objectives as follows:

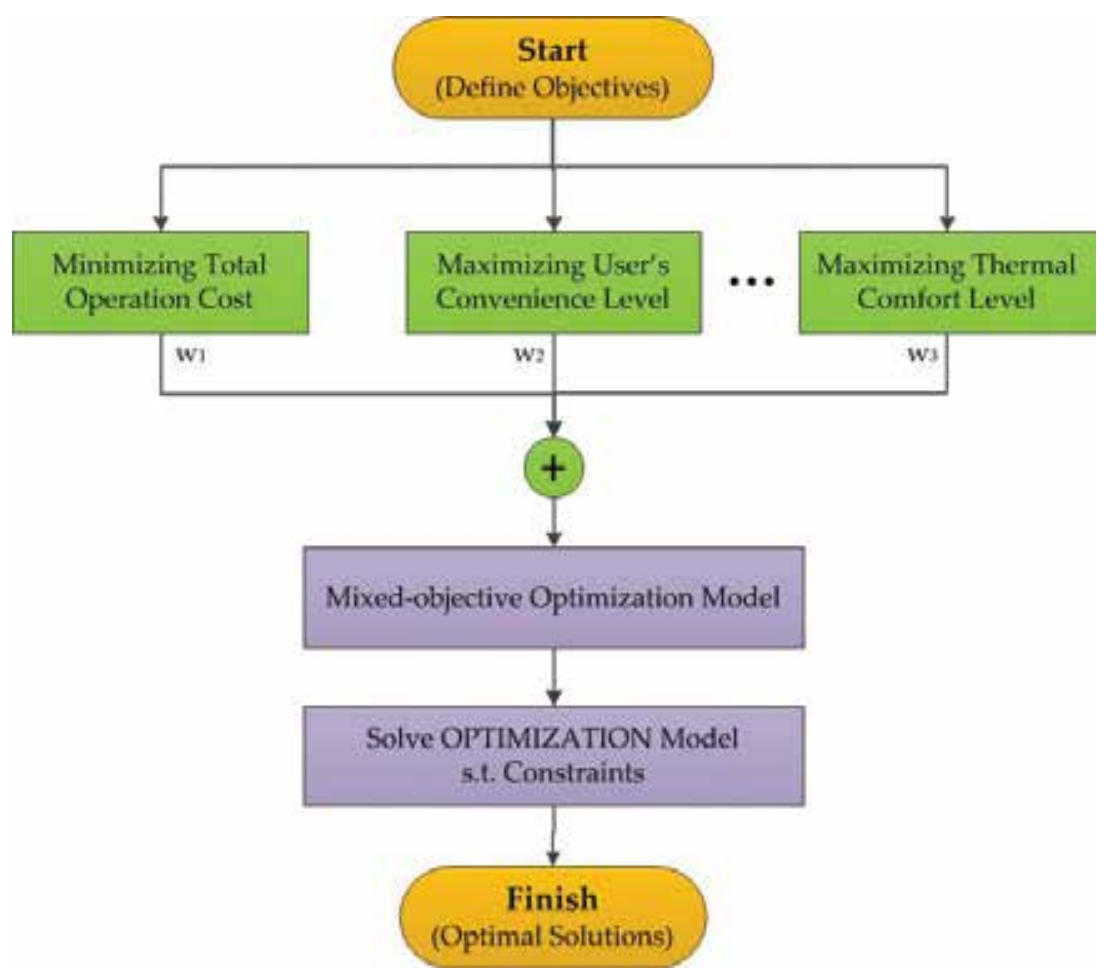

Figure 7. Multi-criteria optimization model.

- Objective 1: minimizing total operation cost

The total cost of operation in short-term for a typical building includes the energy consumption costs as follows:

$$
\min \left\{\operatorname{Cost}=\sum_{h=1}^{T}\left(\begin{array}{l}
\rho_{\text {grid }}(h) \cdot P_{\text {grid }}(h)+\rho_{\text {gas }} \cdot\left(G_{C H P}(h)+G_{\text {aux }}(h)\right) \\
+\delta \cdot\left(\rho_{W T} \cdot P_{W T}(h)+\rho_{P V} \cdot P_{P V}(h)\right)
\end{array}\right)\right\}
$$

where $\rho_{\text {grid }}(h)$ and $P_{\text {grid }}(h)$ are the utility bid and the amount of power exchanged with utility at hour $h$, respectively. $\rho_{\text {gas }}$ is the natural gas price and $G_{C H P}(h)$ and $G_{a u x}(h)$ are the total amount of 
gas consumed by the CHP and the auxiliary boiler at hour $h$, respectively. Likewise, $\rho_{W T}$ and $\rho_{P V}$ are the bids and the hourly output power of the internal RESs such as WT and PV, respectively. $\delta$ is the user's subscription rate denotes the energy share of each resident from the SMG according to ratios of investment.

- Objective 2: maximization of the user's convenience level (UCL)

As mentioned beforehand, all schedulable tasks in a home have their own utilization and preferred time ranges, which can be used as measurement tools for the UCL and satisfaction degree could be obtained when those tasks are executed at different times. To quantify the user's satisfaction level, the following formulation could be introduced:

$$
\max \left\{U C L=\sum_{i=1}^{N} w_{i} \cdot C V_{i}(h)\right\}
$$

where $w_{\mathrm{i}}$ is a significance factor showing the operating priority of task $i$, and $C V_{i}(\mathrm{~h})$ is the degree of convenience experienced by the user when task $i$ is executed at hour $h$ :

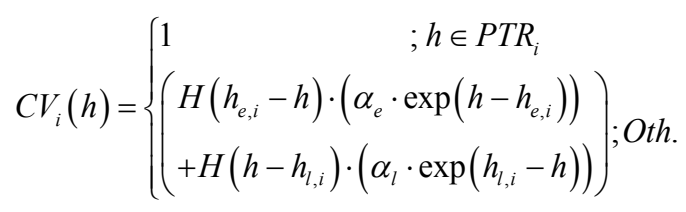

where $\alpha_{e}, \alpha_{l} \in \mathrm{R}^{+}$are the leading coefficients of the natural exponential functions used for controlling the penalty values over the optimization process.

- Objective 3: maximization of the thermal comfort level (TCL)

Thermal comfort for occupants of a building mainly depends on three factors including the indoor air temperature, relative humidity of the environment, and air motion from which the insider air temperature has the greatest effect on TCL. Based on the surveys done on thermal comfort zone of human, it has been found that majority of clothed people feel comfortable in the operative temperature range of $23-27^{\circ} \mathrm{C}$ [14]. Regarding this point, one can measure occupant TCL as follow:

$$
\max \left\{T C L=\sum_{h=1}^{T} C L_{t h}(h)\right\}
$$

where $C L_{t h}(h)$ could be quantified as: 


$$
C L_{\text {th }}(h)=\left\{\begin{array}{rr}
\beta_{c} \cdot \exp \left(T_{\text {indoor }}(h)-T_{\text {set }}+\Delta T_{\text {ther }}\right) & ; T_{\text {indoor }}(h)-T_{\text {set }}<-\Delta T_{\text {ther }} \\
1 & ;\left|T_{\text {indoor }}(h)-T_{\text {set }}\right| \leq \Delta T_{\text {ther }} \\
\beta_{h} \cdot \exp \left(T_{\text {set }}+\Delta T_{\text {ther }}-T_{\text {indoor }}(h)\right) & ; T_{\text {indoor }}(h)-T_{\text {set }}>+\Delta T_{\text {ther }}
\end{array}\right.
$$

where $T_{\text {set }}$ is the user-specified set-point for indoor temperature and $\Delta T_{\text {ther }}$ is the threshold temperature difference. $\beta_{c}, \beta_{h} \in \mathrm{R}^{+}$are also the leading coefficients of the natural exponential functions used for adjusting the penalty values assigned to the undesirable lower and higher temperature differences, respectively.

\subsection{Optimization model for energy and comfort management}

Since optimal energy management of a residential building inherently involves multiple, conflicting and incommensurate objectives as mentioned before, a mixed objective function can be introduced as the model of optimization for coordinated energy and comfort management [13]:

$$
\operatorname{Min}\left\{J=\frac{\text { Cost }}{\xi_{1} \cdot U C L+\xi_{2} \cdot T C L}\right\}
$$

where the weighting coefficients $\zeta_{1}$ and $\zeta_{2}$ denoting the relative significance of TCL and UCL from the user's prospective. The above mentioned optimization problem must be solved considering the following power balance equation together with all other existing constraints for a REM problem:

$$
P_{\text {grid }}(h)+P_{C H P}^{e}(h)+\delta \cdot\left(P_{W T}(h)+P_{P V}(h)\right)-P_{B a t t}(h)=P_{D}^{e}(h)
$$

\section{Coordinated DR and DG management in a sample IES}

In this section of the chapter, a number of computer simulations are presented to show the performance of a typical SEMS for coordinated DR and DG management in an integrated building and SMG system as shown in Figure 8. It is also worthy of note that the algorithm coding and computer simulations are carried out in MATAB/Simulink and General Algebraic Modeling System (GAMS) with Cplex/Dicopt solvers on a core i5 computer with $2430 \mathrm{M}$ processor@ $2.4 \mathrm{GHz}$. 


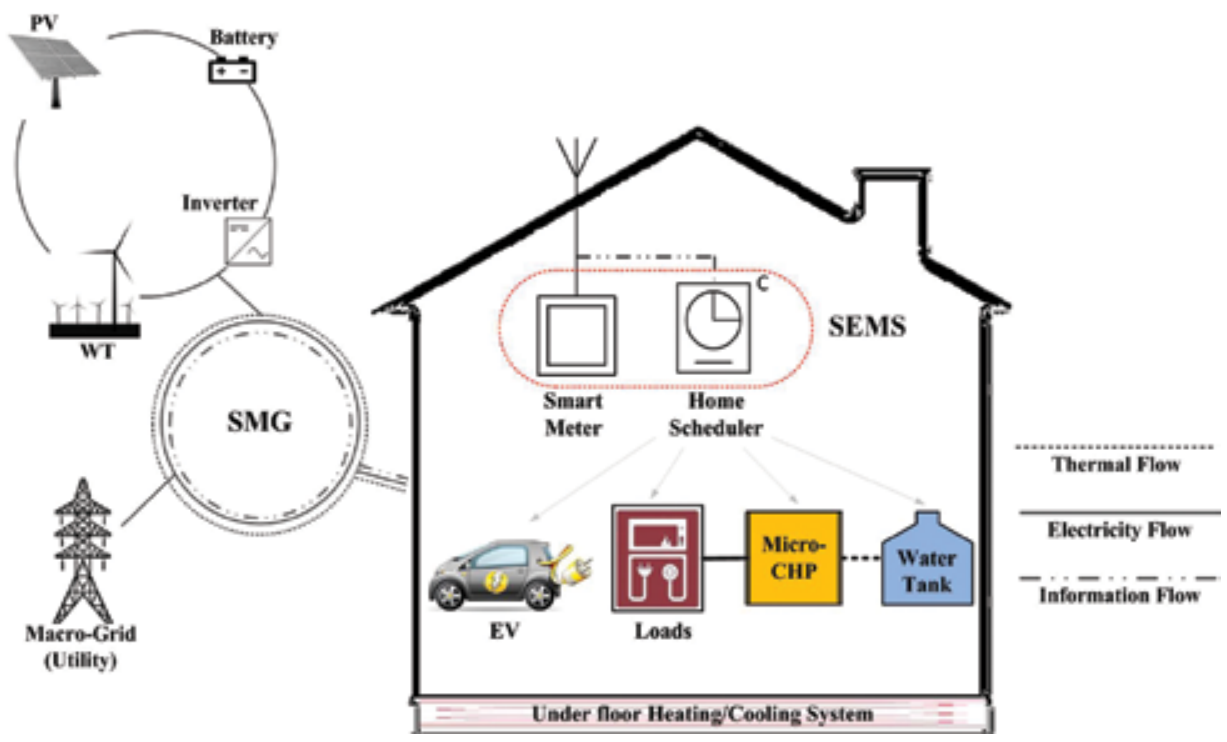

Figure 8. Architecture of a sample IES including building and SMG systems.

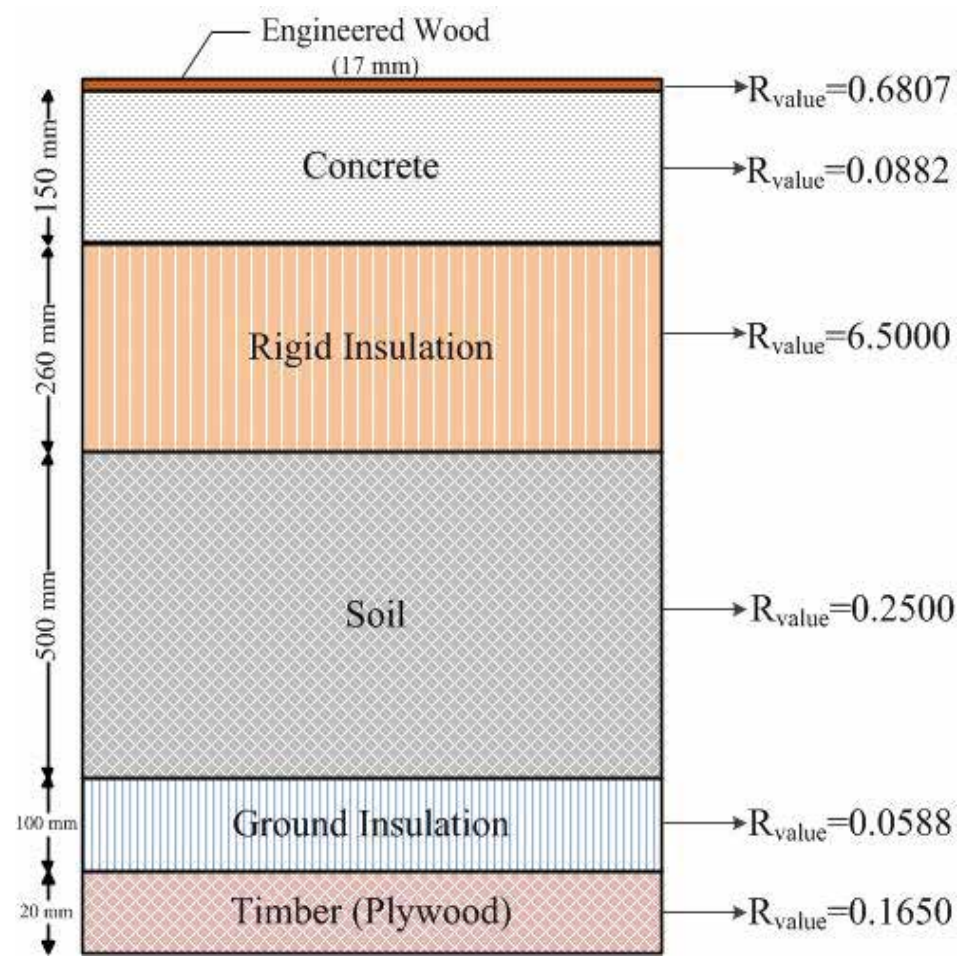

Figure 9. Structural layers of the floor. 
The analyses are conducted for one of the variations of a real single-zone, low-energy building in Sydney (latitude $33.86^{\circ} \mathrm{S}$ and longitude $151.21^{\circ} \mathrm{E}$ ) [16]. The building unit is oriented north, fully exposed to solar insolation and has a floor area of $201.2 \mathrm{~m}^{2}$. The North/South and the East/ West facing walls are also 56 and $28.2 \mathrm{~m}^{2}$, respectively. Double-glazed windows are used on each side of the house with different surface areas. The windows on the North (South) side are $15 \mathrm{~m}^{2}\left(7 \mathrm{~m}^{2}\right)$ while the ones on the East/West sides are $4 \mathrm{~m}^{2}$ with no blinds or shading equipment. The house roof and the walls have similar insulation level $\left(R_{\text {value }}=6.25\right)$. The floor structure for this building unit is also shown in Figure 9. All the smart controllable devices and schedulable loads described in the previous section are also included in the examined IES using the parameters tabulated in the following tables. For the mentioned house, the total internal heat gains is calculated from a load profile of typical household electrical loads and occupancy to be $3.5 \mathrm{~W} / \mathrm{m}^{2}$ of floor area averaged over a 24 -hour period.

To simulate DGs in the proposed IES different realistic models have been used. The windpowered generator is implemented based on a direct-driven, variable-speed, pitch-controlled Morphic SWT20 turbine with a nominal power of $20 \mathrm{~kW}$ at a wind speed of $9 \mathrm{~m} / \mathrm{s}$. Table 2 shows the specification of such system. The PV system is also simulated based on a $0.25 \mathrm{~kW}$ Hyundai mono-crystalline module whose technical data is tabulated in Table 3.

\begin{tabular}{lll}
\hline Parameters & Value & Unit \\
\hline Cut-in wind & 2 & $\mathrm{~m} / \mathrm{s}$ \\
Survival wind & 60 & $\mathrm{~m} / \mathrm{s}$ \\
Swept area & 120 & $\mathrm{~m}^{2}$ \\
Rotor diameter & 12.35 & $\mathrm{~m}$ \\
Nacelle (length $\times$ width $\times$ height) & $2.45 \times 0.7 \times 0.7$ & $\mathrm{~m}$ \\
Weight (complete nacelle, with rotor and blades) & 960 & $\mathrm{~kg}$ \\
\hline
\end{tabular}

Table 2. Wind turbine performance and mechanical data.

\begin{tabular}{lll}
\hline Parameters & Value & Unit \\
\hline Voltage at maximum power & 30.5 & $\mathrm{~V}$ \\
Current at maximum power & 8.2 & $\mathrm{~A}$ \\
Open circuit voltage & 37.5 & $\mathrm{~V}$ \\
Short circuit current & 8.7 & $\mathrm{~A}$ \\
Module efficiency & 15.5 & $\%$ \\
Nominal operating cell temperature & $46 \pm 2$ & ${ }^{\circ} \mathrm{C}$ \\
\hline
\end{tabular}

Table 3. Electrical characteristics of mono-crystalline type solar module. 
The FC co-generation system is also a fusion of Viessmann's highly efficient, boiler-based heating technology and the FC technology of Panasonic whose main specifications are introduced in Table 4. Likewise, the features of the proposed RFH/CS are shown in Table 5.

In our simulation analysis, the ESS is modeled based on a lithium-ion battery pack mounted on a Nissan Leaf electrical vehicle considering the features mentioned in Table 6. Other information such as schedulable tasks specifications and user's preferences during different times is expressed in Table 7.

\begin{tabular}{|c|c|c|c|}
\hline Element & Parameter & Value & Unit \\
\hline \multirow[t]{5}{*}{ Fuel Cell unit } & Electric capacity range & $0.3-1.5$ & $\mathrm{~kW}$ \\
\hline & Ramp capacity & 0.9 & $\mathrm{kWh}$ \\
\hline & Natural gas consumption rate for producing $1 \mathrm{kWh}$ energy & \multicolumn{2}{|c|}{$92.4 \times 10^{-3} \mathrm{~m}^{3} / \mathrm{h}$} \\
\hline & Electrical, thermal efficiency & 30,70 & $\%$ \\
\hline & Weight & 125 & $\mathrm{~kg}$ \\
\hline \multirow[t]{3}{*}{ Aux. boiler } & Thermal capacity range & $4-19$ & $\mathrm{~kW}$ \\
\hline & Efficiency & 86 & $\%$ \\
\hline & Weight (boiler and tank unit) & 170 & $\mathrm{~kg}$ \\
\hline \multirow[t]{5}{*}{ Domestic hot water (DHW) tank } & Total capacity & 200 & liter \\
\hline & Surface area & 1.99 & $\mathrm{~m}^{2}$ \\
\hline & Insulation $R_{\text {value }}(0.04 \mathrm{~m}$ thickness $)$ & 2.818 & $\mathrm{~m}^{2}{ }^{\circ} \mathrm{C} / \mathrm{W}$ \\
\hline & Hot water temperature range & $60-80$ & ${ }^{\circ} \mathrm{C}$ \\
\hline & Inlet water temperature & 10 & ${ }^{\circ} \mathrm{C}$ \\
\hline
\end{tabular}

Table 4. FC-based cogeneration system parameters.

\begin{tabular}{|c|c|c|c|}
\hline Parameters & & Value & Unit \\
\hline Maximum heating (cooling) power & & 2 & $\mathrm{~kW}$ \\
\hline Range of heating COP & & $100-400$ & $\%$ \\
\hline Range of cooling COP & & $100-300$ & $\%$ \\
\hline Temperature range of under floor fluid & & 10,40 & ${ }^{\circ} \mathrm{C}$ \\
\hline Set point temperature & & 25 & ${ }^{\circ} \mathrm{C}$ \\
\hline \multirow[t]{2}{*}{ Comfortable temperature ranges } & Hot weather & $22-28$ & ${ }^{\circ} \mathrm{C}$ \\
\hline & Cold weather & $23-27$ & \\
\hline
\end{tabular}

Table 5. Under floor heating and cooling system specifications. 


\begin{tabular}{lll}
\hline Parameters & Value & Unit \\
\hline Capacity & 24 & $\mathrm{kWh}$ \\
Charge, discharge range & $3.3,3.3$ & $\mathrm{~kW}$ \\
SOC range & $20-80$ & $\%$ \\
Charge, discharge efficiency & 87,90 & $\%$ \\
Dimensions & $1.570 \times 1.188 \times 0.265$ & $\mathrm{~m}$ \\
Weight & 294 & $\mathrm{~kg}$ \\
\hline
\end{tabular}

Table 6. Energy storage device specifications.

\begin{tabular}{llllllc}
\hline Appliance & UTR & PTR (weekday) & PTR (weekend) & LOT & EEC (kWh) & $w_{i}$ \\
\hline Washing machine & $7: 00-21: 00$ & $8: 00-14: 00$ & $13: 00-19: 00$ & 2 & 1 & 1 \\
Dishwasher & $9: 00-22: 00$ & $14: 00-18: 00$ & $17: 00-21: 00$ & 2 & 1.4 & 2 \\
Dryer & $9: 00-21: 00$ & $11: 00-17: 00$ & $16: 00-21: 00$ & 1 & 1.8 & 1 \\
Iron & $1: 00-13: 00$ & $5: 00-7: 00$ & $8: 00-11: 00$ & 1 & 1.1 & 2 \\
Vacuum cleaner & $8: 00-20: 00$ & $9: 00-12: 00$ & $15: 00-19: 00$ & 1 & 0.65 & 2 \\
Microwave & $8: 00-19: 00$ & $11: 00-14: 00$ & $13: 00-16: 00$ & 1 & 0.9 & 3 \\
Rice cooker & $10: 00-20: 00$ & $14: 00-17: 00$ & $16: 00-19: 00$ & 2 & 0.6 & 3 \\
Electric Kettle & $4: 00-12: 00$ & $6: 00-7: 00$ & $8: 00-10: 00$ & 1 & 1 & 3 \\
Toaster & $1: 00-10: 00$ & $6: 00-8: 00$ & $7: 00-9: 00$ & 1 & 0.8 \\
\hline
\end{tabular}

Table 7. Schedulable tasks data and user's preference.

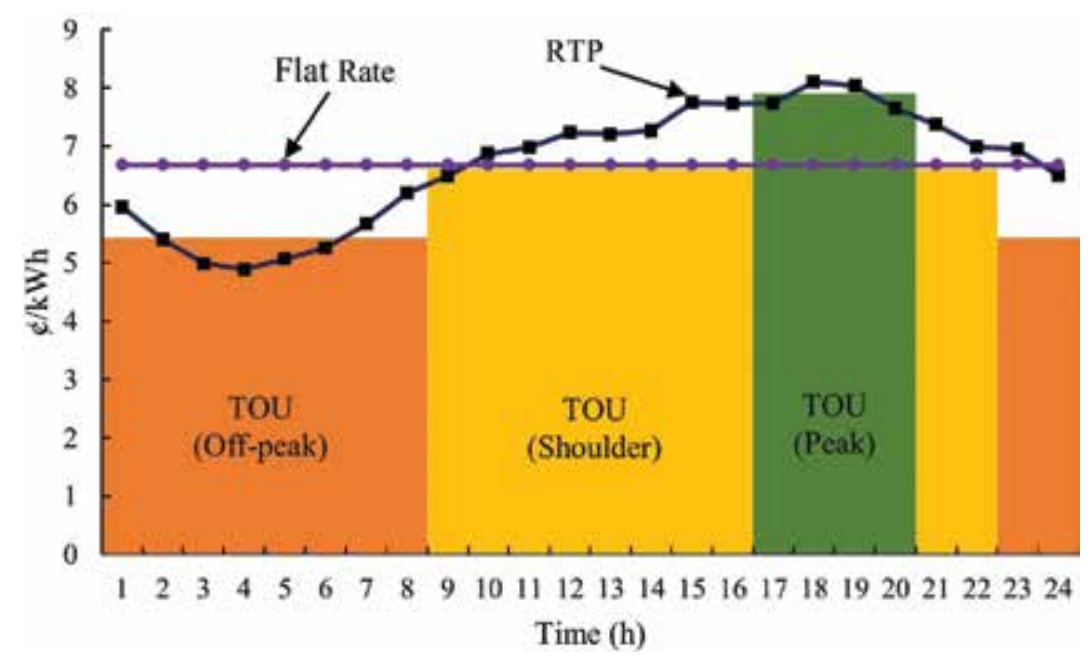

Figure 10. Energy trading schemes. 


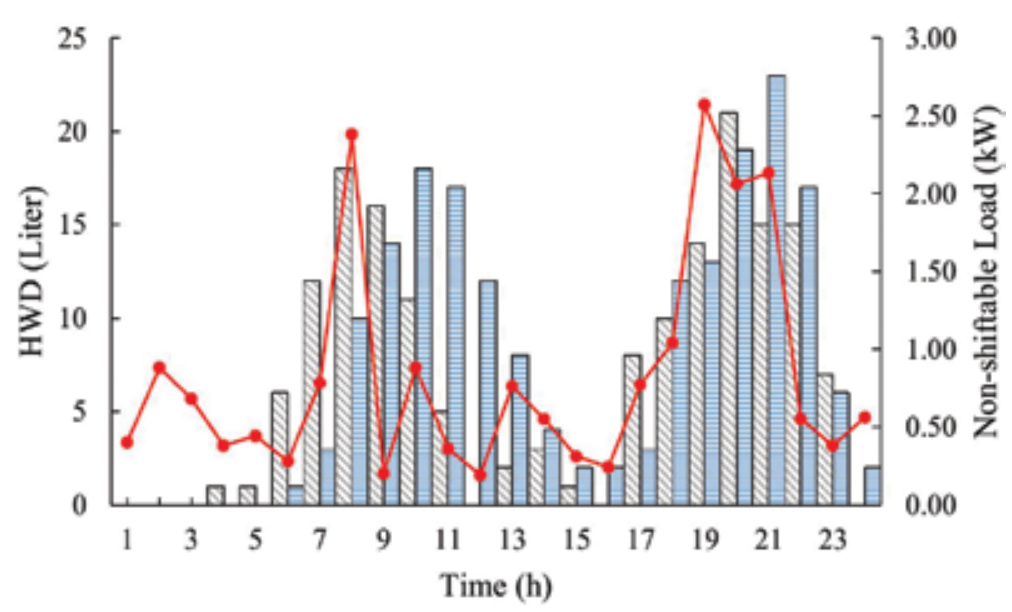

Weckday HWD Weckend HWD $\longrightarrow$ Non-shiftable loads

Figure 11. Non-schedulable load profile and HWD of the examined house.

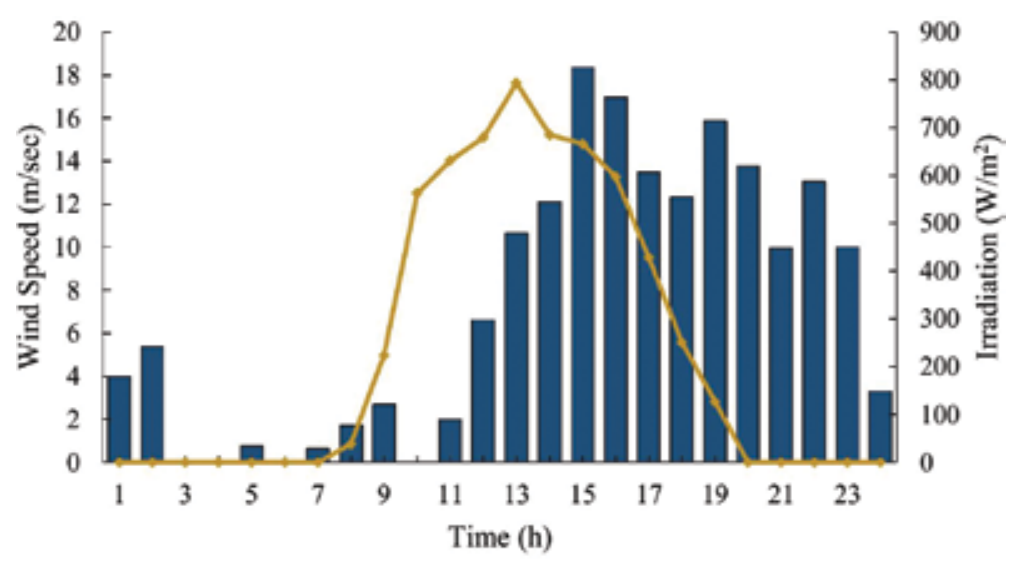

Wind speed —Imadiation

Figure 12. Outdoor wind speed and solar radiation.

In the performed simulations, total power consumption of the building is limited to $5.5 \mathrm{~kW}$ at each time slot. As shown in Figure 10, different energy pricing mechanisms such as flat rate pricing (FLR), time of use tariffs (TOU) and RTP are also studied within the examined IES. The natural gas is also priced as $33 \mathrm{\$} / \mathrm{m}^{3}$. To study the effect of heating and cooling cases, we consider different outdoor air temperatures and conduct a number of simulations in the presence of different EMSs and conditions as shown in Figures 11 and 12.

Table 8, shows a detailed comparison of the performances between the proposed SEMS and a naïve one (NEMS) under different operating conditions. 


\begin{tabular}{|c|c|c|c|c|c|c|c|c|c|c|c|c|c|}
\hline \multirow[t]{3}{*}{ Objective function } & \multirow[t]{3}{*}{ EMS type } & \multicolumn{6}{|c|}{ Hot weather conditions } & \multicolumn{6}{|c|}{ Cold weather conditions } \\
\hline & & \multicolumn{3}{|c|}{ Weekend } & \multicolumn{3}{|c|}{ Weekday } & \multicolumn{3}{|c|}{ Weekend } & \multicolumn{3}{|c|}{ Weekday } \\
\hline & & $\overline{\text { RTP }}$ & TOU & FLR & RTP & TOU & FLR & RTP & TOU & FLR & RTP & TOU & FLR \\
\hline \multirow[t]{2}{*}{ Cost $(\$)$} & SEMS & 267.94 & 268.10 & 286.10 & 257.06 & 263.20 & 279.41 & 232.78 & 265.65 & 265.80 & 227.42 & 238.87 & 7261.29 \\
\hline & NEMS & 240.10 & 243.07 & 255.75 & 231.98 & 238.27 & 261.52 & 189.32 & 214.18 & 216.82 & 182.83 & 187.75 & 5206.11 \\
\hline \multirow[t]{2}{*}{ UCL } & SEMS & 100.00 & 100.00 & 100.00 & 100.00 & 100.00 & 100.00 & 100.00 & 100.00 & 100.00 & 100.00 & 100.00 & 100.00 \\
\hline & NEMS & 39.13 & 52.63 & 26.32 & 30.89 & 39.59 & 38.90 & 24.08 & 32.39 & 20.30 & 18.20 & 24.08 & 24.37 \\
\hline \multirow[t]{2}{*}{ TCL } & SEMS & 83.69 & 83.83 & 84.10 & 85.25 & 87.67 & 86.30 & 88.43 & 88.49 & 89.56 & 89.66 & 92.40 & 87.46 \\
\hline & NEMS & 85.30 & 85.67 & 86.14 & 86.55 & 89.41 & 88.07 & 89.64 & 90.75 & 91.05 & 91.16 & 94.59 & 89.22 \\
\hline \multirow[t]{2}{*}{$\mathrm{J}$} & SEMS & 1.46 & 1.46 & 1.55 & 1.39 & 1.40 & 1.50 & 1.24 & 1.41 & 1.40 & 1.20 & 1.24 & 1.39 \\
\hline & NEMS & 1.93 & 1.76 & 2.27 & 1.98 & 1.85 & 2.06 & 1.66 & 1.74 & 1.95 & 1.67 & 1.58 & 1.81 \\
\hline \multirow[t]{2}{*}{ Calc. time $(\mathrm{s})^{1}$} & SEMS & 4.15 & 4.03 & 3.83 & 4.05 & 3.74 & 3.63 & 3.42 & 3.34 & 3.52 & 3.86 & 3.71 & 3.50 \\
\hline & NEMS & 2.85 & 2.66 & 2.74 & 2.75 & 2.71 & 2.39 & 2.18 & 2.39 & 2.04 & 2.23 & 2.20 & 2.14 \\
\hline
\end{tabular}

${ }^{1}$ Based on a PC with an Intel i5-2430M chip running Windows 7(64 bit) with GAMS and Cplex/Dicopt solvers.

Table 8. Performance comparison between smart and naïve energy management systems under different operating conditions.

As observed in Table 8, economic task scheduling is achieved by using a NEMS, however user's preferences are not considered as another key point. On the contrary, cost-effective operation of in-home devices as well as comfort-aware task scheduling is perfectly done by the SEMS which use advanced controlling features. It is clearly understood from the numerical results that the mixed objective function $(J)$ is improved about $26 \%$ by the use of RTP-based SEMS in different time frames of a hot weather condition and about $21 \%$ in similar condition but with a TOU-based SEMS. The performance of the proposed EMS is also getting better compared to the NEMS in a cold weather condition mainly due to the Sun's effect on the cooling load of the building. SEMS not only reduces energy consumption cost of the residential unit, but also satisfies optimal task scheduling and provides a thermal comfort zone for inhabitants. It can be also observed from the simulation results that the optimal performances of the mentioned EMSs are highly affected by several parameters such as pricing mechanism and time frames. As an example the running cost of the IES decreases once RTP is utilized and it increases when FLR pricing is applied. In a like manner, the operating cost of the IES is higher in a hot season compared to the one in a cold season. The same trend can be observed for weekends and weekdays. Due to the presence of occupants and their pattern of consumption, the energy cost of a building unit is slightly higher in weekends compared to weekdays. As illustrative examples, a number of related computer simulations are also presented to get more insight into the performance of SEMS. Figure 13 illustrates the performance of the proposed residential SEMS as applied to the heating/cooling scenarios for the given building in different weather conditions [17]. Optimal coordinated DR and DG management for the studied IES by using SEMS is also indicated in Figure 14. This figure shows the optimal operation of smart 
household devices, FC-based micro-CHP unit and battery along with the amount of power exchange between the building and the utility for the given demand profiles in a typical hot weather condition [18].
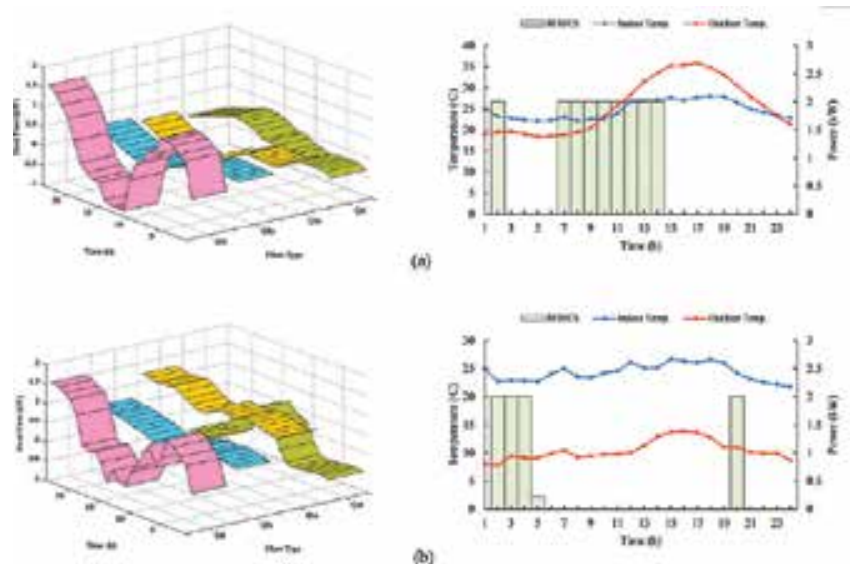

Figure 13. Different heat flows and optimal operations of RFH/CS based on the thermal demand and user's comfort level: (a) hot weather conditions and (b) cold weather conditions.

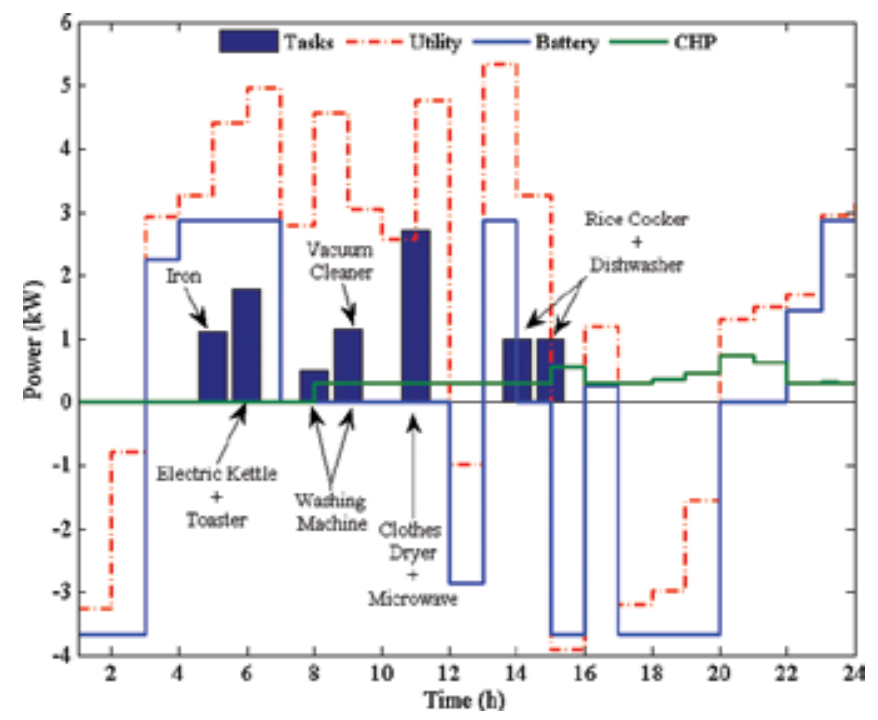

Figure 14. Coordinated DR and DG management using SEMS.

Based on the temperature differences between different nodes in indoor air and outdoor environment, heat can be easily transferred via the building structures and affects the thermal behavior of the residential unit. As can be seen in Figure 13, the building captures the heat from both direct and indirect solar radiation on the exposed surfaces (such as walls and the 
roof on the sunny side) during the hot summer days and releases that heat later in the day. For this reason, $\mathrm{RFH} / \mathrm{CS}$ operates more in the cooling mode to satisfy the desired body comfort. In a cold winter day the RFH/CS must be operated in the heating mode to keep the indoor temperature within the comfort range, although the internal and external heat gains of the building assist the heating process. Moreover, as observed from the temperature profiles in heating and cooling cases, the thermal constraints are almost respected and the comfort zone chosen by the customer is satisfied in terms of valid temperature ranges.

It is also observed from Figure $\mathbf{1 4}$ that through optimal coordinated management of DR programs and DG units, not only the cost of energy consumption is reduced, but also comfortable thermal and electrical zones are guaranteed. It should be also noted that most of the electrical demand is supplied by the utility during hours when the RTP is relatively low (e.g., 3:00-7:00 and 13:00-15:00). In the same time frames, the batteries are also charged with lower cost. During other times of the day when the energy demand is growing and the electricity prices are higher, distributed generators (such as energy storage devices and co-generation unit) produce more electricity so as to meet the load economically, and make more profits by selling the surplus of energy to the utility. DR programs for optimal task scheduling are also activated in a way to satisfy user's preferences.

\section{Conclusion}

In this chapter, a framework was outlined for coordinated DR and DG management in an integrated building and smart micro-grid system. A SEMS for scheduling loads at the demandside and domestic controllable units at the supply-side was also described, mathematically modeled and validated. The proposed SEMS incorporated the conventional energy use management principles represented in DSM and DERs programs and merged them in an integrated framework that simultaneously addressed permanent energy savings, demand reductions, and temporary peak load mitigations. It also captured different key modeling aspects including heat transfer and thermal dynamics of a residential building unit, schedulable tasks attributes, and DG units' specifications. Moreover, it integrated different RESs, storage systems, and domestic thermo-electrical systems to provide a given cost reduction and comfort levels according to the customer needs.

\section{Author details}

Amjad Anvari-Moghaddam ${ }^{1 *}$, Ghassem Mokhtari' ${ }^{2}$ and Josep M. Guerrero ${ }^{1}$

*Address all correspondence to: aam@et.aau.dk

1 Department of Energy Technology, Aalborg University, Aalborg East, Denmark

2 CSIRO, Brisbane, Australia 


\section{References}

[1] Tushar M. H. K., Assi C., Maier M., Uddin M. F. Smart microgrids: optimal joint scheduling for electric vehicles and home appliances. IEEE Trans. Smart Grid. 2014; 5: 239-250.

[2] Anvari-Moghaddam A., Monsef H., Rahimi-Kian A. Cost-effective and comfort-aware residential energy management under different pricing schemes and weather conditions. Energy Build. 2015; 86: 782-793. DOI: 10.1016/j.enbuild.2014.10.017.

[3] Kailas A., Cecchi V., Mukherjee A. A survey of communications and networking technologies for energy management in buildings and home automation. J. Comput. Netw. Commun. 2012; 2012: 1-12. DOI: 10.1155/2012/932181.

[4] Anvari-Moghaddam A., Seifi A. R. A comprehensive study on future smart grids: definitions, strategies and recommendations. J. N. C. Acad. Sci.. 2011; 127: 28-34. DOI: 10.7572/2167-5880-127.1.28.

[5] Wang Z., Wang J. Self-healing resilient distribution systems based on sectionalization into microgrids. IEEE Trans. Power Systems. 2015; 30: 3139-3149.

[6] Anvari-Moghaddam A. Global Warming Mitigation Using Smart Micro-Grids. Global Warming-Impacts and Future Perspective, Prof. Dr. Bharat Raj Singh (Ed.), ISBN: 978-953-51-0755-2, InTech, Rijeka, Croatia, 2012. DOI: 10.5772/48204.

[7] Bian D., Pipattanasomporn M., Rahman S. A human expert-based approach to electrical peak demand management. IEEE Trans. Power Delivery. 2015; 30: 1119-1127.

[8] Chowdhury S., Chowdhury S. P., Crossley P. Microgrids and Active Distribution Networks. The Institution of Engineering and Technology, Michael Faraday House, Six Hills Way, Stevenage, Herts., SG1 2AY, UK. 2009. 297p. DOI: 10.1049/PBRN006E.

[9] Anvari-Moghaddam A, Seifi A. R., Niknam T., Alizadeh Pahlavani M. R.. Multiobjective operation management of a renewable micro grid with back-up micro turbine/ fuel cell/battery hybrid power source. Energy. 2011; 36(11): 6490-6507.

[10] Ekanayake J., Jenkins N., Liyanage K., Wu J., Yokoyama A. Smart grid: technology and applications. Wiley; 2012. 320p. ISBN: 978-1-119-96909-9.

[11] Guerrero J. M., Vasquez J. C., Matas J., de Vicuña L. G., Castilla M. Hierarchical control of droop-controlled AC and DC microgrids - a general approach toward standardization. IEEE Trans. Ind. Electron. 2011; 58(1): 158-172. DOI: 10.1109/TIE.2010.2066534.

[12] Electric Power Research Institute (EPRI). Integrating smart distributed energy resources with distribution management systems. EPRI-Power Delivery \& Utilization; 2012. 14 p. ID: 1024360. 
[13] Anvari-Moghaddam A., Rahimi-Kian A., Monsef H. Optimal smart home energy management considering energy saving and a comfortable lifestyle. IEEE Trans. Smart Grid. 2015; 6: 324-332. DOI: 10.1109/TSG.2014.2349352.

[14] ASHRAE. Handbook-Fundamentals, American Society of Heating, Refrigerating and Air-Conditioning Engineers Publishers, Inc., Atlanta, GA, USA. 2001.

[15] Du Bois D., Du Bois E. F. A formula to estimate the approximate surface area if height and weight be known. Arch. Int. Med. 1916; 17: 863-871.

[16] Bambrook S. M., Sproul A. B., Jacob D. Design optimisation for a low energy home in Sydney. Energy Build. 2011; 43: 1702-1711. DOI: 10.1016/j.enbuild.2011.03.013.

[17] Anvari-Moghaddam A., Rahimi-Kian A., Monsef H., Vasquez J. C., Guerrero J. M. Optimized energy management of a single-house residential micro-grid with automated demand response. IEEE PES PowerTech Conference, June 29-July 2, Eindhoven, 2015.

[18] Anvari-Moghaddam A., Vasquez J. C., Guerrero J. M. Load shifting control and management of domestic microgeneration systems for improved energy efficiency and comfort. 41st Annual Conference of the IEEE Industrial Electronics Society, November 9-12, Yokohama, Japan, 2015. 

Chapter 3

\title{
Hierarchical Control for DC Microgrids
}

\author{
Ahmed Mohamed \\ Additional information is available at the end of the chapter \\ http://dx.doi.org/10.5772/63986
}

\begin{abstract}
In this chapter, the design and control of DC microgrids will be discussed. Depending on the time and bandwidth requirements, microgrid controllers can be categorized to primary local controllers (LC) and secondary microgrid central controllers (MGCC). The functions of the two categories of controllers will be presented and explained, using simulations and hardware experiments. In addition, the design of the power electronic converters linking the various resources and loads within the DC microgrid to the common DC bus, as well as the converters used to connect the microgrid to the main grid, will be presented. An example of the interaction of the MGCC with the controller of the main grid will be investigated. This chapter is intended to give a practical overview of the design and control of DC microgrids.
\end{abstract}

Keywords: control, DC microgrids, inverter, primary microgrid control, secondary microgrid control

\section{Introduction}

Electric power systems are undergoing profound and radical changes triggered by the imperative to fulfill two objectives: (1) increase the power system resilience and (2) combat global warming. Resilience, as articulated by the Presidential Policy Directive 21 [1], refers to "the ability to withstand and recover from deliberate attacks, accidents, or naturally occurring threats or incidents." In order to approach the first objective, consumers must be equipped with generators and storage elements in order to supply their loads locally during blackouts. In other words, the bulk power system will be divided into many local energy networks, namely microgrids, which are interconnected through the main power network during 
normal operating conditions, but can island themselves and operate independently when a fault occurs. Microgrids increase the overall efficiency of the system by satisfying part of their load locally, reducing the amount of power imported over long transmission lines. The effort to achieve the second objective is aided by increasing renewable energy generation. There is a wide consensus that if we are to realize the full environmental, societal, and economic benefits of microgrid deployment, while also improving resilience, microgrids must be based on renewable energy.

According to the U.S. Department of Energy, a microgrid is "a group of interconnected loads and distributed energy resources (DERs) with clearly defined electrical boundaries that acts as a single controllable entity with respect to the electric utility grid." A microgrid can be connected to the grid, in a grid-connected mode, or independent from the grid, in an islanded mode. Operation and control of microgrids have been given genuine attention in the literature. Each of the individual resources and controllable loads needs a controller. These controllers are referred to as local or resource controllers. In addition, a microgrid central controller (MGCC) is needed to act as a coordinator/supervisor to the local controllers (LCs).

The speed and bandwidth requirements significantly vary between LCs and MGCCs. LCs need to be faster since they have to deal with current and voltage commands and measurements, whereas MGCCs take a supervisory role in managing the power flow of the assets and loads within the microgrid and between the microgrid and the main grid. In addition, MGCCs fix any errors, for example, frequency deviation that may result from primary control $[2,3]$. Tertiary control of microgrids refers the layer of control that extends beyond the boundaries of a single microgrid. It coordinates the interaction between various microgrids in close vicinity and the main grid. A virtual power plant whether owned by the utility company or a third party, aggregating some microgrids in a given geographic area, can be considered an example of tertiary control. This layer of control is typically considered as a part of the main grid's control $[2,4]$ and will be out of the scope of this chapter. However, by the end of the chapter, the interaction of the MGCC with the main grid will be highlighted.

DC microgrids offer several advantages over AC microgrids [5, 6]. Electronic devices, such as computers, routers, and electronic lights (either fluorescent or LED), represent a high percent of the electric load in many buildings today. Moreover, variable speed drives (VFD) are increasingly used for electric motors. A DC environment is found to be a more convenient way to deliver power to these loads to assure reliability and redundancy. DC networks do not need AC to DC conversion for every electronic device, which has a significant impact on the efficiency. DC can reduce the losses associated with switch-mode supplies and uninterruptible power supplies. Furthermore, incorporating DC microgrids has the benefit of superior compatibility of the DC power with renewable energy generators, for example, photovoltaics (PV), electric vehicles, and energy storage systems (ESSs) [7-10].

In this chapter, we will focus on the design of primary and secondary control techniques for DC microgrids. Computer simulations and hardware testing will be used to verify the presented techniques. The simulation results were obtained using MATLAB/Simulink and the SimPowerSystems sublibrary. 


\section{Microgrid structure}

The DC microgrid under study is assumed to be dependent mainly on the sustainable energy sources, as shown in Figure 1. The microgrid is connected to the main grid, so that it can operate in a grid-connected mode. Moreover, it includes an ESS, so that it can operate in an islanded mode during blackout/brownout conditions. During the grid-connected mode, power can be drawn either from the main grid or from the ESS in case the locally generated renewable energy is not enough to satisfy the load demand.

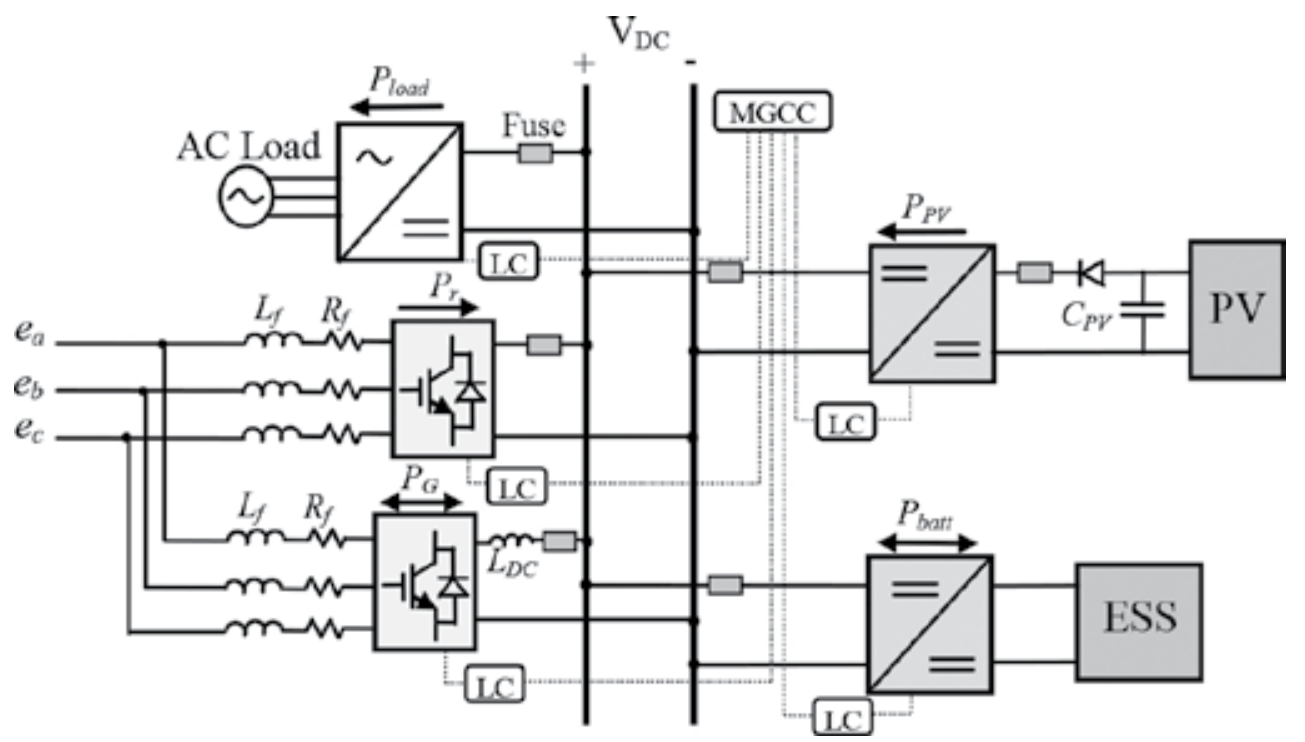

Figure 1. DC microgrid architecture and control hierarchy.

Since the microgrid is based on renewable energy, certain features had to be maintained to assure efficient integration of the renewable resources, such as efficient and reliable loadfeeding capability and full controllability of voltage and power flow among the various buses in the system. The connectivity of the DC microgrid to the main grid should allow voltage regulation on the DC side. Furthermore, it should allow bidirectional power flow between AC and DC sides, depending on the desired mode of operation.

Specifically, a fully controlled rectifier was used to tie the DC network to the AC grid while working at unity power factor. This rectifier is dedicated to regulating the voltage on the DC bus in the grid-connected mode. Therefore, it enables unidirectional power flow from the main grid to the DC microgrid. Alternatively, one of the other resource converters, for example, the bidirectional battery charger, must be responsible for regulating the DC bus voltage. A fully controlled bidirectional AC-DC converter was used to control the active/reactive power exchange with the main grid. It employs a vector decoupling control technique, which enables independent control of the active and reactive power in both directions. Each converter is controlled via a LC. A MGCC communicates with the LCs and coordinates their operation. 


\section{Energy link integration}

\subsection{Converters and control design}

The ESS and PV will be linked to the common DC bus of the microgrid through DC-DC converters. Boost converter is commonly used to interface renewable energy sources yielding DC voltage to the DC microgrid. In case of PV systems, a controlled boost converter shall serve two functionalities: (1) it steps up the output voltage of the PV system to be compatible with the DC bus voltage and (2) it regulates the output voltage or power, for example, corresponding to a predefined maximum power point tracking algorithm. The boost converter topology may be slightly modified when used as an interface for PV systems in a DC microgrid. For instance, since the DC bus may already possess high capacitance (i.e., owing to the other converters connected to the same bus), the boost converter capacitor can be omitted, resulting in a discontinuous instantaneous output current. A solution to maintain continuous output current is to synchronize multiple DC-DC converters (i.e., interleaved converters) [11, 12].

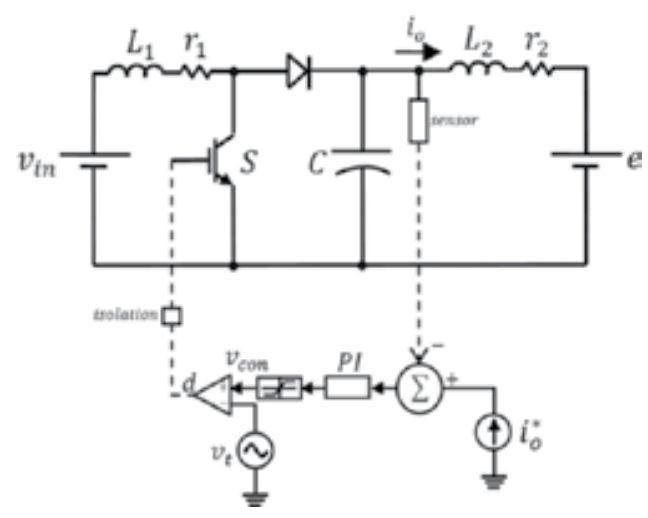

Figure 2. The proposed inductively coupled boost converter topology for fuel cells integration into a DC ZEDS.

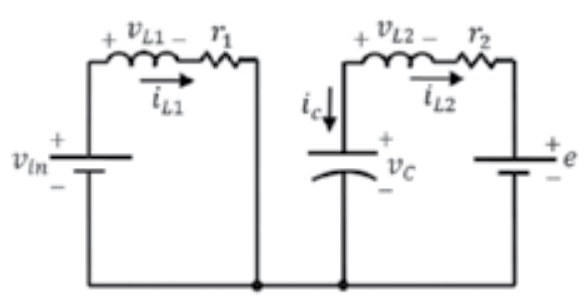

(a)

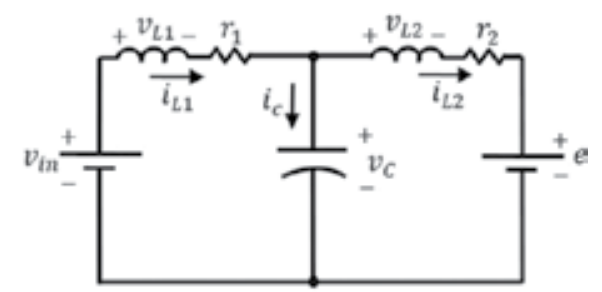

(b)

Figure 3. The ON and OFF states of the DC-DC converter with output L-filter: (a) $(0<t \leq D T s)$ and (b) $(D T s<t \leq T)$.

Another approach to solve the problem of discontinuity in the output current is by adding an L-filter to the output side of the converter, as shown in Figure 2. The added inductance assures 
continuous conduction of the output current. The configurations of the circuit during the $\mathrm{ON}$ and OFF states of the switches are shown in Figure 3, where the parameter $D$ in the caption refers to the duty cycle and $T_{s}$ refers to the switching time.

The small-signal mathematical model of this converter can be obtained using the state-space averaging technique. The state-space model during the interval $\left(0<t \leq D T_{s}\right)$ is [11]

$$
\left[\begin{array}{c}
i \cdot{ }_{L 1} \\
i_{L 2} \\
v_{c}^{\cdot}
\end{array}\right]=\left[\begin{array}{ccc}
\frac{-r_{1}}{L_{1}} & 0 & \frac{-1}{L_{1}} \\
0 & \frac{-r_{2}}{L_{2}} & \frac{1}{L_{2}} \\
0 & \frac{-1}{C} & 0
\end{array}\right]\left[\begin{array}{l}
i_{L 1} \\
i_{L 2} \\
v_{C}
\end{array}\right]+\left[\begin{array}{cc}
\frac{1}{L_{1}} & 0 \\
0 & \frac{-1}{L_{2}} \\
0 & 0
\end{array}\right]\left[\begin{array}{l}
v_{i n} \\
e
\end{array}\right]
$$

whereas the state-space model during the interval $\left(D T_{s}<t \leq T\right)$ is

$$
\begin{gathered}
{\left[\begin{array}{c}
i_{L 1} \\
i_{L 2} \\
v_{C}
\end{array}\right]=\left[\begin{array}{ccc}
\frac{-r_{1}}{L_{1}} & 0 & 0 \\
0 & \frac{-r_{2}}{L_{2}} & \frac{1}{L_{2}} \\
\frac{1}{C} & \frac{-1}{C} & 0
\end{array}\right]\left[\begin{array}{l}
i_{L 1} \\
i_{L 2} \\
v_{C}
\end{array}\right]+\left[\begin{array}{cc}
\frac{1}{L_{1}} & 0 \\
0 & \frac{-1}{L_{2}} \\
0 & 0
\end{array}\right]\left[\begin{array}{l}
v_{i n} \\
e
\end{array}\right]} \\
i_{0}=i_{L 2}
\end{gathered}
$$

Using the state-space averaging technique,

$$
\left[\begin{array}{c}
\left\langle i_{L 1}\right\rangle \\
\left\langle i_{L 2}\right\rangle \\
\left\langle v_{c}\right\rangle
\end{array}\right]=\left[\begin{array}{ccc}
\frac{-r_{1}}{L_{1}} & 0 & \frac{-d}{L_{1}} \\
0 & \frac{-r_{2}}{L_{2}} & \frac{1}{L_{2}} \\
\frac{(1-d)}{C} & \frac{-1}{C} & 0
\end{array}\right]\left[\begin{array}{c}
\left\langle i_{L 1}\right\rangle \\
\left\langle i_{L 2}\right\rangle \\
\left\langle v_{c}\right\rangle
\end{array}\right]+\left[\begin{array}{cc}
\frac{1}{L_{1}} & 0 \\
0 & \frac{-1}{L_{2}} \\
0 & 0
\end{array}\right]\left[\begin{array}{c}
\left\langle v_{i n}\right\rangle \\
\langle e\rangle
\end{array}\right]
$$

If we consider a small-signal perturbation, the large-signal state-space equations will be 


$$
\left[\begin{array}{l}
0 \\
0 \\
0
\end{array}\right]=\left[\begin{array}{ccc}
\frac{-r_{1}}{L_{1}} & 0 & \frac{-d}{L_{1}} \\
0 & \frac{-r_{2}}{L_{2}} & \frac{1}{L_{2}} \\
\frac{(1-d)}{C} & \frac{-1}{C} & 0
\end{array}\right]\left[\begin{array}{c}
I_{L 1} \\
I_{L 2} \\
V_{c}
\end{array}\right]+\left[\begin{array}{cc}
\frac{1}{L_{1}} & 0 \\
0 & \frac{-1}{L_{2}} \\
0 & 0
\end{array}\right]\left[\begin{array}{c}
V_{\text {in }} \\
E
\end{array}\right]
$$

whereas the small-signal state-space set of equations will be

$$
\left[\begin{array}{c}
\hat{i}_{\dot{L} 1} \\
\hat{i}_{L 2} \\
\hat{v}_{c}^{\cdot}
\end{array}\right]=\left[\begin{array}{ccc}
\frac{-r_{1}}{L_{1}} & 0 & \frac{-D}{L_{1}} \\
0 & \frac{-r_{2}}{L_{2}} & \frac{1}{L_{2}} \\
\frac{(1-D)}{C} & \frac{-1}{C} & 0
\end{array}\right]\left[\begin{array}{l}
\hat{i}_{L 1} \\
\hat{i}_{L 2} \\
\hat{v}_{c}
\end{array}\right]+\left[\begin{array}{cc}
\frac{1}{L_{1}} & \frac{-V_{c}}{L_{1}} \\
0 & 0 \\
0 & \frac{-I_{L 1}}{C}
\end{array}\right]\left[\begin{array}{c}
\hat{v}_{i n} \\
\hat{d}
\end{array}\right]
$$

where

$$
i_{L 1}=I_{L 1}+\hat{i}_{L 1}, i_{L 2}=I_{L 2}+\hat{i}_{L 2}, v_{c}=V_{c}+\hat{v}_{c}, v_{i n}=V_{i n}+\hat{v}_{i n}, e=E+\hat{e}, d=D+\hat{d}
$$

\subsection{Energy link integration results and discussion}

A prototype system was designed and implemented in hardware to examine the performance of the inductively coupled boost converter. The set of equations described in Section 3.1 was

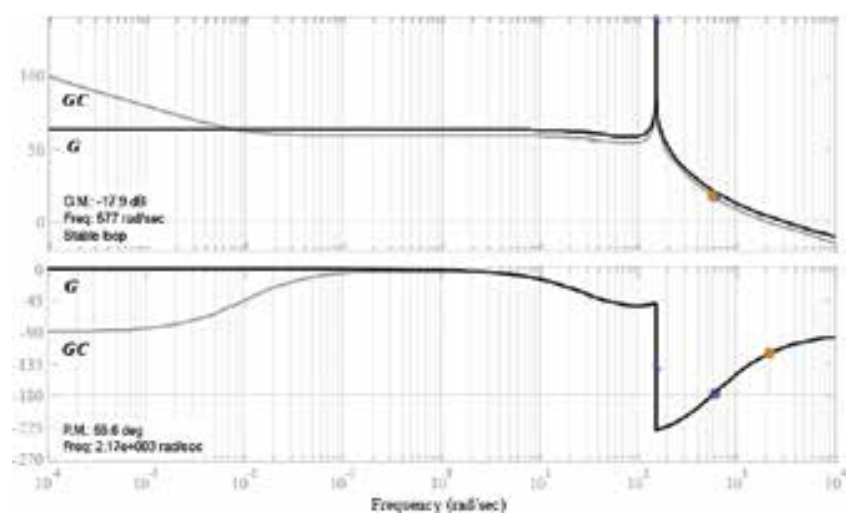

Figure 4. Bode plots of the developed controller. 
used to design a closed-loop proportional-Integral (PI) controller. The digital signal processing board dSPACE 1104 was used to control the converter in real time. The switching frequency for the converter was $5 \mathrm{kHz}$. The parameters of the implemented converter prototype were as follows: $L_{1}$ is $2.2 \mathrm{mH}, L_{2}$ is $24 \mathrm{mH}, r_{1}$ is $0.06 \mathrm{ohm}, r_{2}$ is $0.84 \mathrm{ohm}$, and $C$ is $4800 \mu \mathrm{f}$. The Bode plots of the open-loop and closed-loop responses for the developed controller are given in Figure 4 .

Results for the inductively coupled boost converter are shown in Figure 5. The output current reference changed from 1 to $3 \mathrm{~A}$ after $5 \mathrm{~s}$. It can be seen that the output current is continuous, and the ripple is as small as $2 \%$, which means a high power quality injected to the DC microgrid.

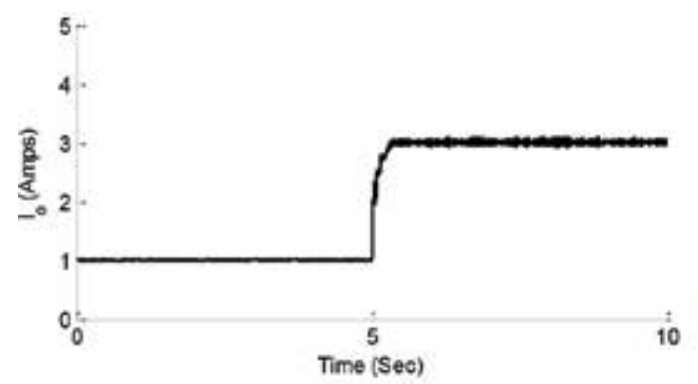

(a)

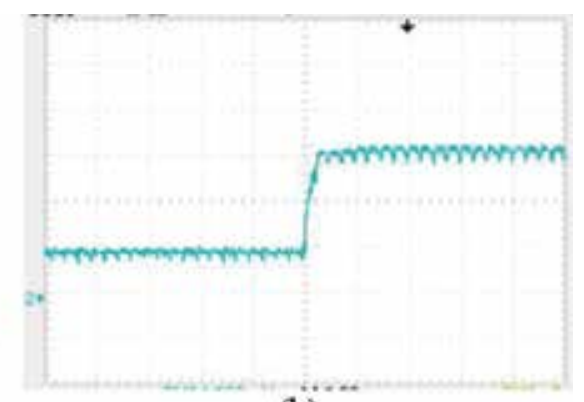

(b)

Figure 5. Results for (inductively coupled boost converter): (a) simulation results and (b) experimental results (the same scale: $1 \mathrm{~A}$ /division).

\section{Microgrid to main grid connectivity}

\subsection{DC bus voltage regulation}

\subsubsection{Converter description and mathematical modeling}

A fully controlled three-phase rectifier will be used for coupling the DC network to the AC grid. In our case study, we designed and implemented the rectifier such that it regulates the voltage of the DC bus, while being able to operate at unity power factor. This was achieved through a vector decoupling control technique and sinusoidal pulse width modulation (SPWM).

Vector decoupling SPWM control is based on converting the voltages and currents from the three-phase abc frame of references to the $\mathrm{d}-\mathrm{q}$ frame of references. Even though mathematical models for the system have been derived, PI controllers were implemented to control the rectifier rather than model-based control due to its relative simplicity and effectiveness. However, the mathematical models play an important role in decoupling the vectors, which is essential to achieve independent $P / Q$ control. The three-phase SPWM rectifier circuit and its single-phase equivalent are shown in Figure 6 [13]. 


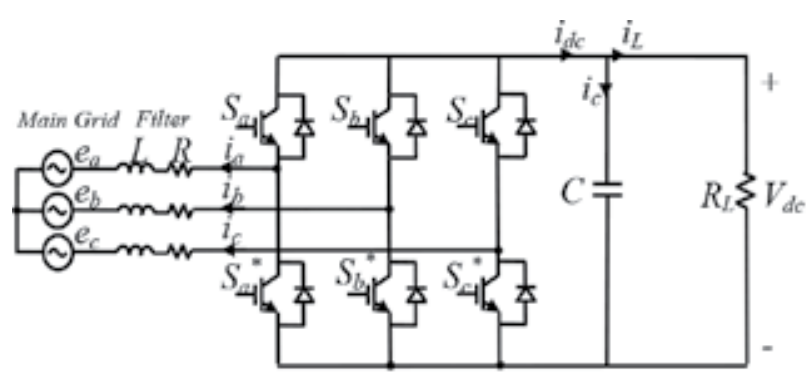

(a)

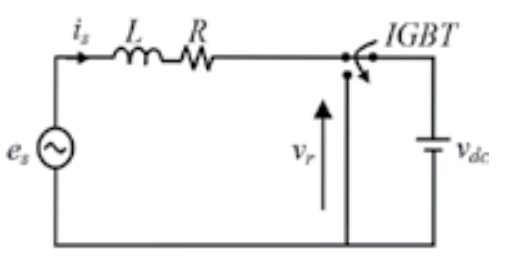

(b)

Figure 6. The implemented three-phase SPWM rectifier: (a) circuit diagram and (b) single-phase equivalent.

The voltage equation is

$$
e_{s}=R i_{s}+L \frac{d i_{s}}{d t}+v_{r}
$$

where

$e_{s}$ is the source voltage

$i_{s}$ is the source current

$v_{r}$ is the converter input voltage

$R, L$ Resistance and inductance of the boosting inductor, respectively.

$$
\begin{gathered}
L \frac{d i_{d e}}{d t}-w L i_{q e}+R i_{d e}=e_{d e}-v_{d e} \\
L \frac{d i_{q e}}{d t}-w L i_{d e}+R i_{q e}=e_{q e}-v_{q e}
\end{gathered}
$$

where $w$ is the angular frequency of the voltage source in radians.

The rectifier should instantaneously draw enough input power to satisfy the sum of the load demand and the charging rate of the capacitor energy, to maintain fast voltage control. Neglecting the thermal and switching device losses, the power balance between the AC input and the DC output is as follows:

$$
P=\frac{3}{2}\left(e_{d e} i_{d e}+e_{q e} i_{q e}\right)=v_{d c} i_{d c}
$$


where $v_{d c}$ and $i_{d c}$ are the DC output voltage and current, respectively.

On the DC output side,

$$
i_{d c}=-C \frac{d v_{d c}}{d t}-i_{L}
$$

where $i_{L}$ is the load current. From Eqs. (10) and (11),

$$
\frac{3}{2}\left(e_{d e} i_{d e}+e_{q e} i_{q e}\right)=-C v_{d c} \frac{d v_{d c}}{d t}-v_{d c} i_{L}
$$

Inspecting Eq. (12), we can see that the system is nonlinear with regard to $v_{d c}$. From Eqs. (8), (9), and (11), a complete state-space modeling of the system is given by

$$
\left[\begin{array}{c}
\dot{i}_{d e} \\
\dot{i}_{q e} \\
\dot{v}_{d c}
\end{array}\right]=\left[\begin{array}{c}
-\frac{R}{L} i_{d e}-w i_{q e} \\
-\frac{R}{L} i_{q e}+w i_{d e} \\
-\frac{3}{2 C v_{D C}}\left(e_{d e} i_{d e}+e_{q e} i_{q e}\right)+\frac{i_{L}}{C}
\end{array}\right]+\left[\begin{array}{cc}
\frac{1}{L} & 0 \\
0 & \frac{1}{L} \\
0 & 0
\end{array}\right]\left[\begin{array}{l}
e_{d e}-v_{d e} \\
e_{q e}-v_{q e}
\end{array}\right]
$$

\subsubsection{Vector decoupling technique}

Two nested loops including three PI controllers were implemented to achieve DC voltage as well as input current control. The outer loop is for controlling the DC bus voltage, while the inner loop is for current control. Due to the vector transformation from abc to $\mathrm{d}-\mathrm{q}$ frame of references, the controller deals with three DC signals, which help eliminate steady-state errors in the developed PI controllers.

In order to completely decouple the $\mathrm{d}$ and q components and achieve independent $P$ and $Q$ control, the decoupling terms $\left(w L i_{d e}\right)$ and $\left(w L i_{q e}\right)$ were included while calculating the rectifier's input voltages for $V_{r q}^{c o n t}$ and, $V_{r q}^{\text {cont }}$ respectively. These voltages are the modulation signals for the SPWM technique. The equations used in building the controller are given by Eq. (14):

$$
\begin{aligned}
& v_{r q}{ }^{c o n t}=w L i_{d e}+e_{q e}-R i_{q e}-K_{p} \cdot\left[i_{d e}{ }^{r e f}-i_{d e}\right]-K_{i} \cdot \int\left[i_{d e}{ }^{r e f}-i_{d e}\right] d t \\
& v_{r d}{ }^{\text {cont }}=-w L i_{q e}-R i_{q e}-K_{p} \cdot\left[i_{d e}{ }^{r e f}-i_{d e}\right]-K_{i} \cdot \int\left[i_{d e}{ }^{r e f}-i_{d e}\right] d t
\end{aligned}
$$

In Figure 7, a layout for the developed controller is shown. 


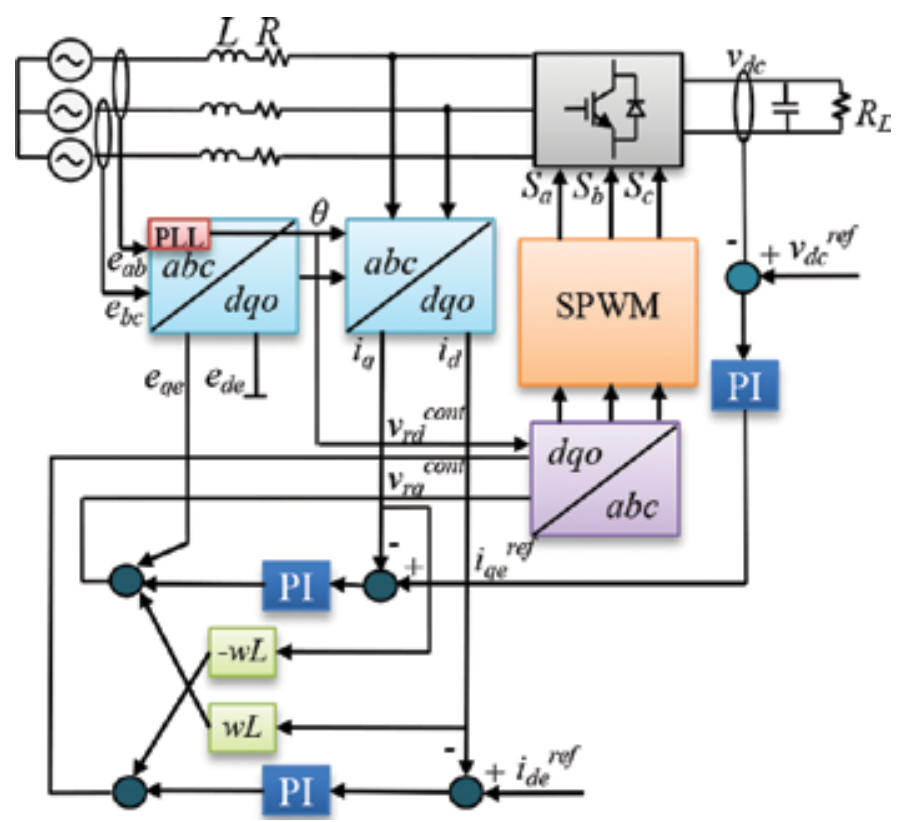

Figure 7. A block diagram of the vector decoupling control implemented on the controlled rectifier.

The controller has the capability to control the active and reactive power independently, and hence, it can be easily set up to operate at unity power factor by adjusting the desired $i_{d e}$ to zero. In mathematical terms, this is represented in Eqs. (15) and (16) as follows [13]:

$$
\begin{aligned}
& P(t)=\frac{3}{2}\left[e_{q e}(t) \cdot i_{q e}(t)+e_{d e}(t) \cdot i_{d e}(t)\right] \\
& Q(t)=\frac{3}{2}\left[e_{d e}(t) \cdot i_{q e}(t)-e_{q e}(t) i_{d e}(t)\right]
\end{aligned}
$$

\subsection{Bidirectional energy transfer}

DC microgrids may draw or inject power to the grid, depending on the local generation/ demand ratio. Therefore, a bidirectional converter must be put in place to enable such energy transfer. For instance, during times of surplus energy, that is, when power from the PV system is greater than the local load, the power can be injected to the grid if the price for electricity is high and/or the battery is fully charged. On the other hand, power may be drawn by the DC microgrid to cover load deficiencies. The vector decoupling control technique discussed earlier in this chapter was utilized here to enable independent active and reactive power control. For the bidirectional converter, the topology is modified by adding an L-filter $(L)$ on the DC side, as shown in Figure 8. Moreover, the DC voltage controller in Figure 7 is replaced by a current controller, as shown in Figure 9 [14]. 


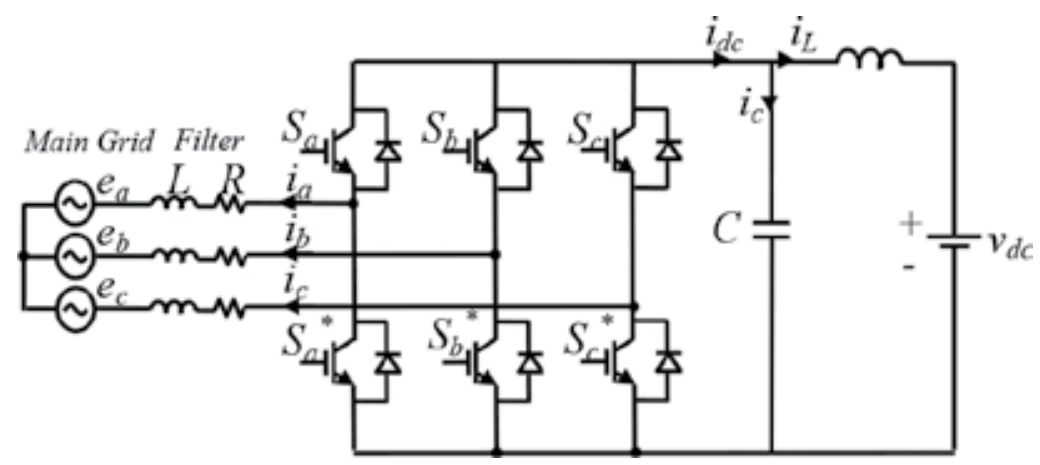

Figure 8. Circuit diagram of the implemented three-phase bidirectional AC-DC/DC-AC converter.

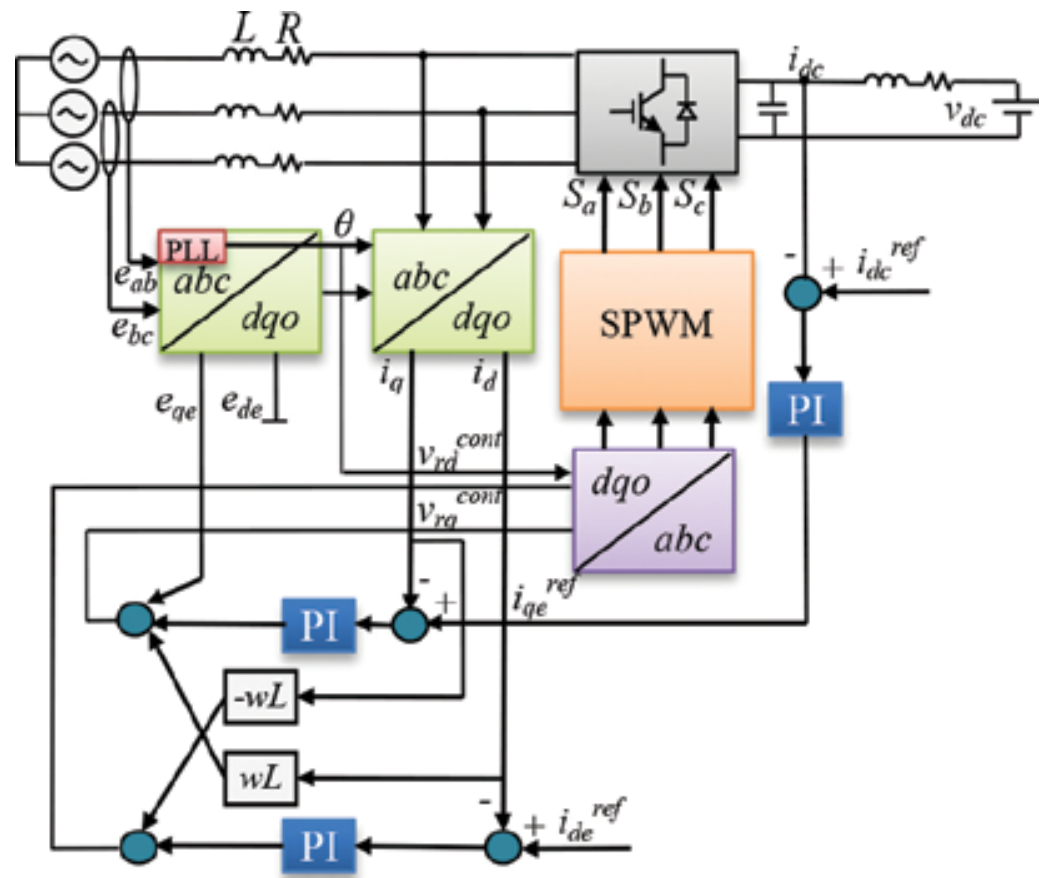

Figure 9. A block diagram of the vector decoupling control implemented on the bidirectional converter.

This controller may be looked at as a means to control the voltage across the L-filter inductor. Corresponding to any given $i_{D C}^{r e f}$ value, the controller adjusts the phase of the modulating SPWM signals for the switching devices, until the desired amount of transferred power is achieved. The DC current is positive when the power flows from the main grid to the microgrid, and vice versa. Therefore, the sign of $i_{q e}^{\text {ref }}$ determines the mode of operation for the bidirectional converter such that it is in the rectifier mode when the sign is positive, and the inverter mode when it is negative. In both modes of operation, the controller allows independent $Q$ control, 
including unity power factor operation by setting $i_{d e}^{r e f}$, which is responsible for the reactive power, to zero.

\subsection{Grid connectivity results and discussion}

Both the unidirectional converter (i.e., the controlled rectifier used for DC bus voltage regulation) and the bidirectional converter were implemented in hardware and simulated in MATLAB/Simulink. The switching frequency for both the converters was $8.04 \mathrm{kHz}$, and the sampling time was $0.3 \mathrm{~ms}$. Voltage and current sensors were deployed to receive feedback from the various nodes of the system [14]. Several experiments were conducted to test the response of the converters under steady state as well as transient operating conditions. The hardware and simulation results will be presented for the various case studies.

\subsubsection{Steady-state performance}

The first experiment aimed at testing the steady-state response of the rectifier while operating at unity power factor. Results of this experiment are shown in Figure 10. As can be seen in the figure, the current and voltage waveforms are in phase, and the DC bus voltage is regulated at $300 \mathrm{~V}$. The DC current is positive, which according to our notation, means that the power is flowing from the main grid to the DC microgrid.

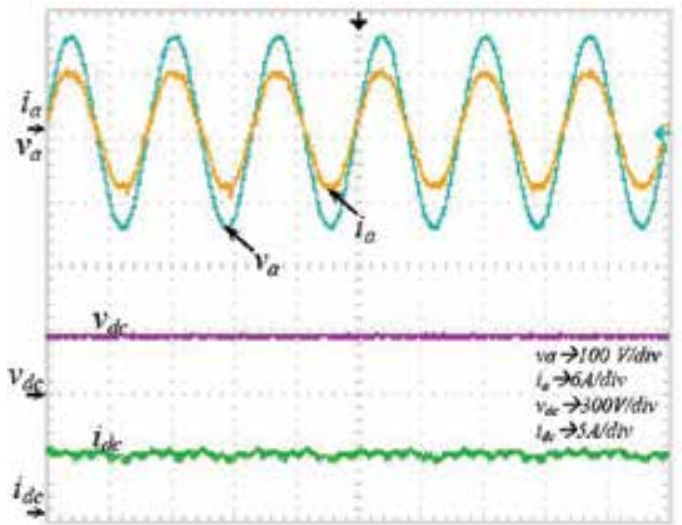

(a)
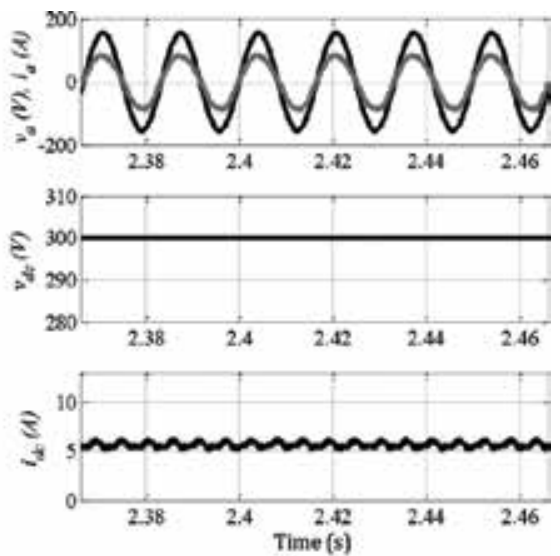

(b)

Figure 10. Unity power factor operation of the controlled rectifier: (a) experimental results and (b) simulation results (AC current factorized by 10).

\subsubsection{Transient performance}

Two experiments were conducted to examine the performance of the developed rectifier under transient operating conditions, namely (1) a step change in the load demand and (2) a step change in the DC microgrid voltage. Figures 11 and 12 depict the results for these two experiments, respectively. 


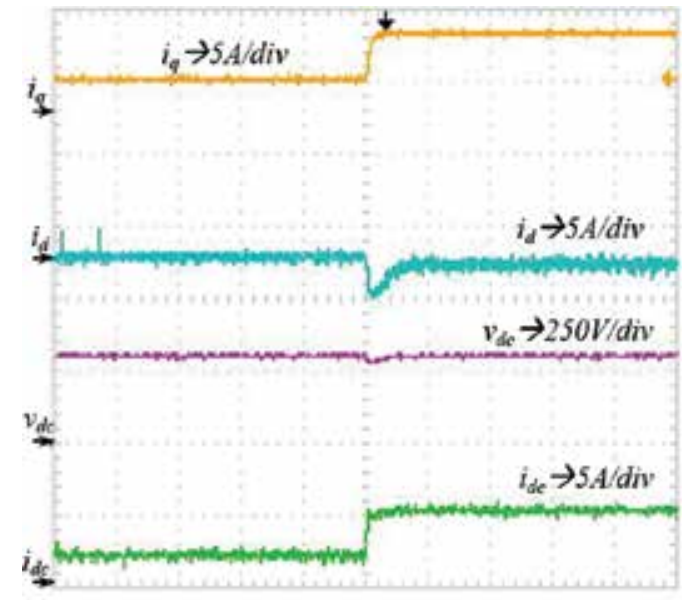

(a)
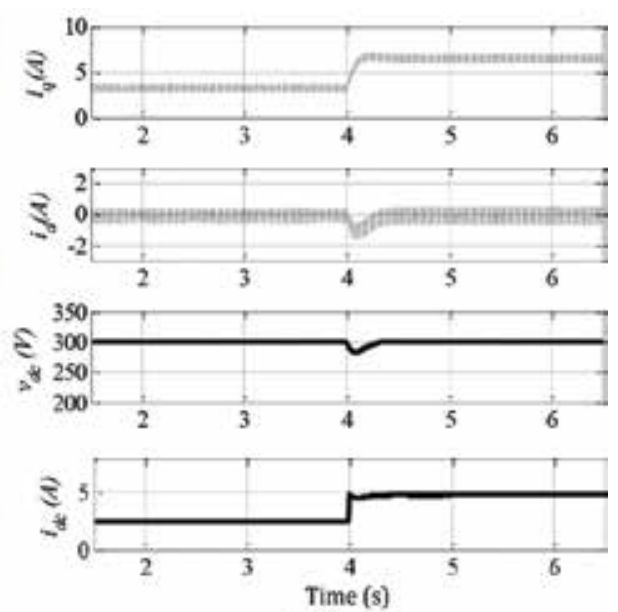

(b)

Figure 11. Controlled rectifier's response to a load step change: (a) experimental results and (b) simulation results.

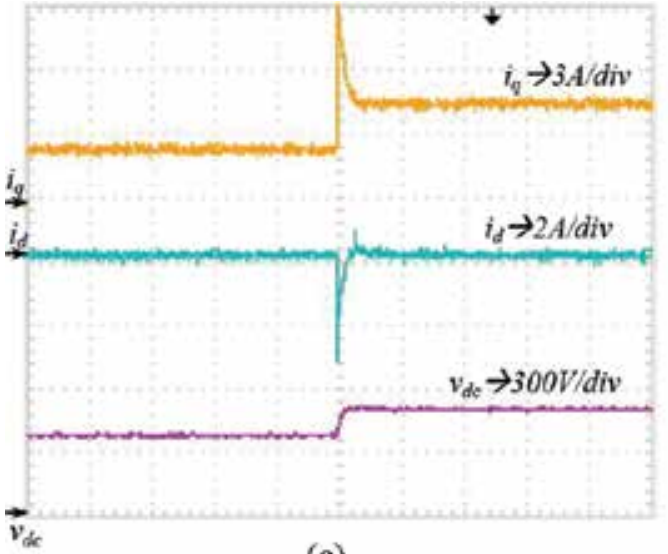

(a)
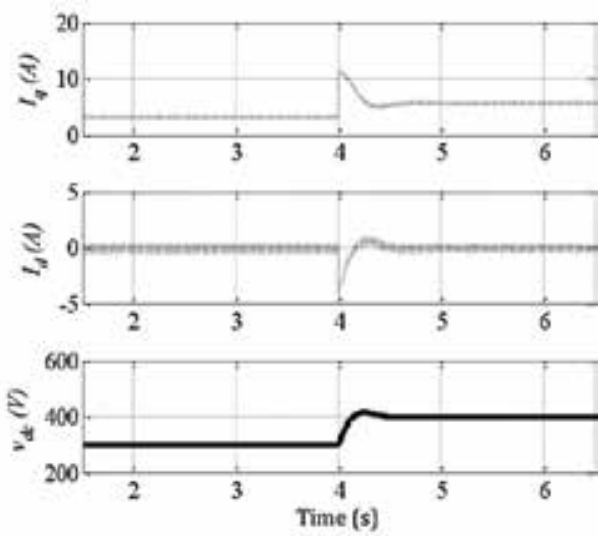

(b)

Figure 12. Controlled rectifier's response to a change in the output voltage: (a) experimental results and (b) simulation results.

For the first experiment, whose results are shown in Figure 11, the DC load was suddenly changed from 0.72 to $1.5 \mathrm{~kW}$. The results show that the converter is capable of responding to the change in the load by increasing the value of $i_{q}$. Moreover, it maintains unity power factor operation, since the value of $i_{d}$, which is responsible for reactive power, decays back to zero shortly after the load step change. The DC voltage encounters a small undershoot because of the load change, while the DC current increases to satisfy the load.

In the second experiment, $v_{D C}^{r e f}$ was increased to represent a sudden change in the DC bus voltage. The rectifier reaches the new voltage set point in $\sim 200 \mathrm{~ms}$. The value of active power 
drawn from the grid increases to satisfy the increase in active power demand on the DC microgrid, which is caused by the increased DC voltage. The converter achieves the required voltage by automatically increasing $i_{q}$, as shown in Figure 12. Meanwhile, $i_{d}$ encounters some transient conditions but returns to zero to maintain unity power factor operation.

\subsubsection{Bidirectional power flow results}

The bidirectional converter was implemented using an L-filter value of $24 \mathrm{mH}$. This filter, which encompasses $0.9 \mathrm{ohm}$ internal resistance, was used to improve the overall total harmonic distortion of the converter and achieve smooth current control. Several experiments were conducted to test the response of the converter under several step changes in the desired power and its direction.

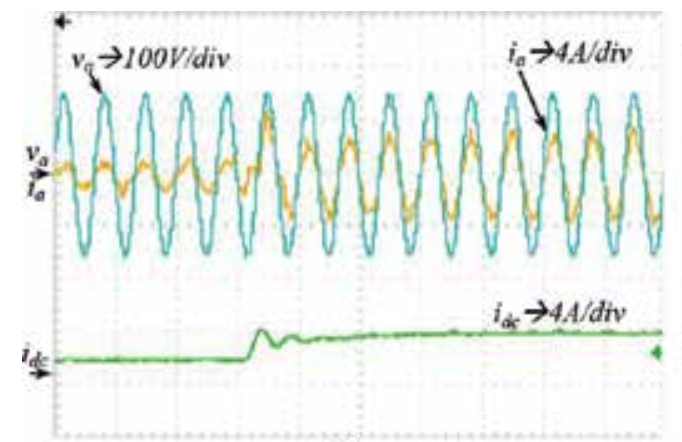

(a)
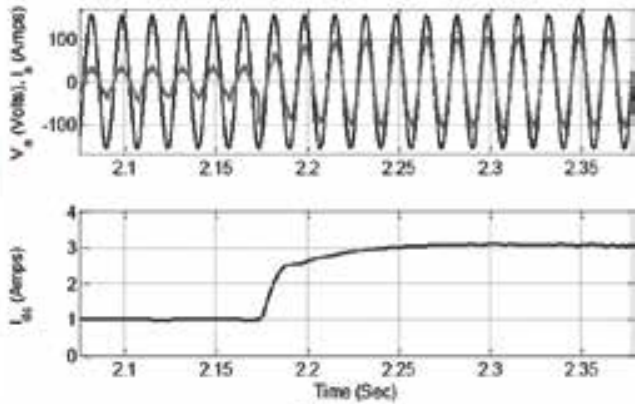

(b)

Figure 13. Controlled bidirectional response to DC current reference change from 1 to $3 \mathrm{~A}$ : (a) experimental results and (b) simulation results (AC current factorized by 10).

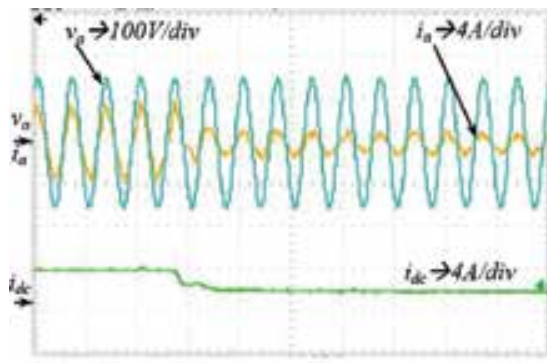

(a)
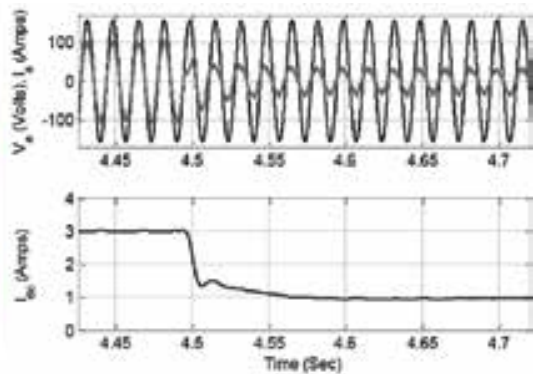

(b)

Figure 14. Controlled bidirectional converter response to DC current reference change from 3 to 1 A: (a) experimental results and (b) simulation results (AC current factorized by 10 ).

The first experiment (see Figure 13) involved a step change in the reference current from 1 to $3 \mathrm{~A}$, while operating in the rectifier mode (i.e., the power flows from the main grid to the DC 
microgrid). Inspecting Figure 13, it can be seen that the converter succeeds in corresponding to the step change in the current reference within a few cycles. The results of a reverse experiment, in which the current reference was decreased from 3 to $1 \mathrm{~A}$, are shown in Figure 14. Both experiments verify the applicability of the developed converter. It is worth mentioning that the experimental results match the simulation results. This assures the credibility of the simulation model, which can be used for analyses that may not be easily performed experimentally, for example, fault analysis.

To examine the converter in the inverter mode, the current reference was changed from -3 to -1 A. The negative sign refers to the inverter mode of operation. Figure 15 shows the results for this experiment. It can be seen that the converter achieves the desired output current, and that the AC current and voltage waveforms are $180^{\circ}$ out-of-phase corresponding to the negative active power direction and zero reactive power.

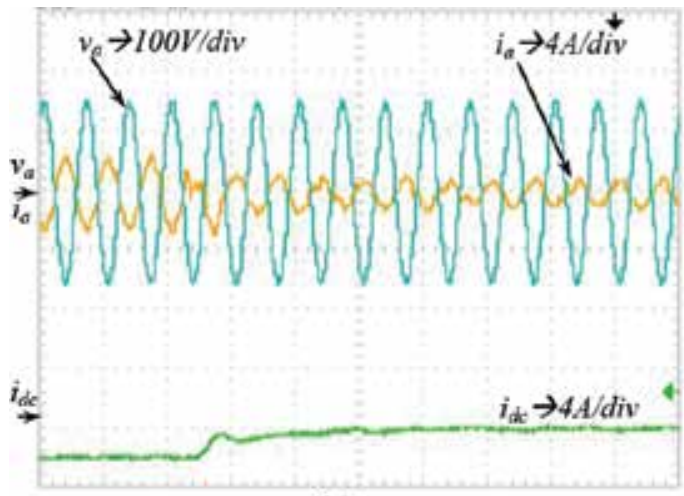

(a)
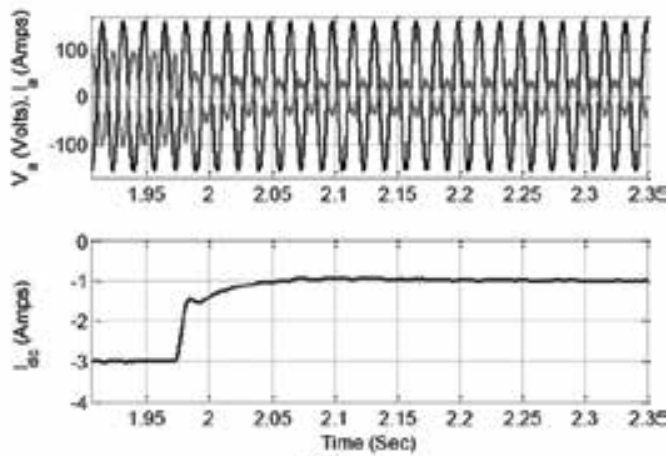

(b)

Figure 15. Controlled bidirectional converter response to DC current reference change ( -3 to $-1 \mathrm{~A})$ : (a) experimental results and (b) simulation results (AC current factorized by 10).

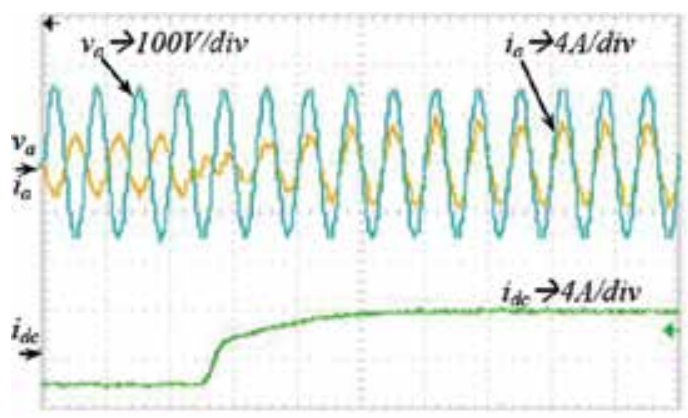

(a)
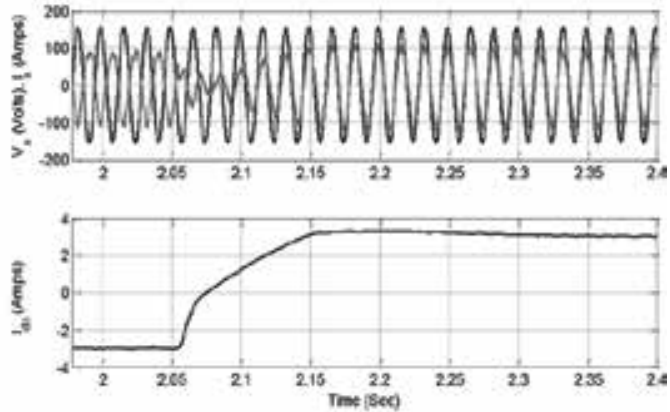

(b)

Figure 16. Controlled bidirectional response to DC current reference change ( -3 to $3 \mathrm{~A}$ ): (a) experimental results and (b) simulation results (AC current factorized by 10). 
In the developed bidirectional converter, the power can flow in both directions, that is, from $\mathrm{AC}$ to DC or from DC to AC. Two experiments were conducted to test the ability of the converter to change the direction of the power instantaneously, while maintaining unity power factor operation. Figure $\mathbf{1 6}$ shows the results of an experiment in which the current reference was changed from -3 to $3 \mathrm{~A}$. This means that the current was flowing from the DC microgrid to the main grid and suddenly reversed its direction. The simulation and experimental results show that the converter was able to control the current and achieve the required step change. Figure 17 shows the case when the current reference was changed from 3 to $-3 \mathrm{~A}$. The results of both experiments show that the converter can smoothly change its mode of operation, from the rectifier to the inverter mode, and vice versa.

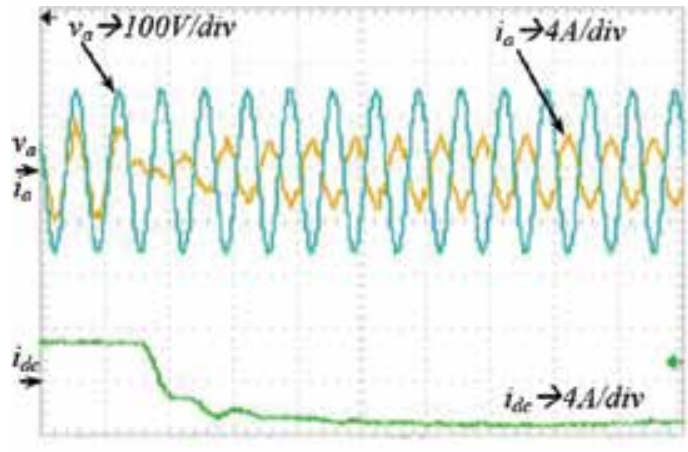

(a)
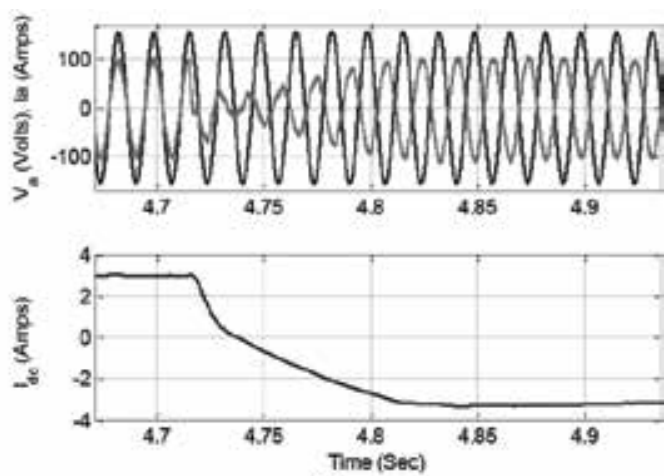

(b)

Figure 17. Controlled bidirectional converter response to DC current reference change ( 3 to $-3 \mathrm{~A})$ : (a) experimental results and (b) simulation results (AC current factorized by 10).

\section{Microgrid central control}

The MGCC communicates not only with the LCs to coordinate their operation but also with the controller of the main grid. The main function of the LC is local voltage and current control of the converter that they are associated with. The functions of the secondary controller are optimal microgrid control, for example, energy cost minimization, broadcasting active and reactive power set points, and islanding detection and operation $[2,15,16]$.

In order to examine the operation of MGCC, an experiment was conducted. Four AC generators were used to form an AC network. The generators were interconnected through transmission line models. The DC microgrid described earlier within this chapter was connected to the main AC grid at the AC point of common coupling (PCC), as shown in Figure 18. The DC microgrid has the ability to draw or inject $P$ and $Q$ to and from the grid. In this experiment, the DC microgrid was regulating the voltage at the PCC. A unity power factor load of $0.7 \mathrm{~kW}$ was initially connected to the PCC, as shown in Figure 19. The DC microgrid was commanded to receive $100 \mathrm{~W}$ and 0 Vars. The AC grid satisfied both the AC load and DC microgrid 
demands. The steady-state voltage at PCC was $0.94 \mathrm{pu}$, while the voltage on the DC bus was $1 \mathrm{pu}$. After $20 \mathrm{~s}$, following this initial state, the DC microgrid MGCC received a request from the main grid to inject the total amount of demanded power on the AC side [17]. Consequently, the voltage improved to $1.02 \mathrm{pu}$. The controlled rectifier regulating the voltage on the DC bus maintains a voltage of 1 pu after a transient period of around $6 \mathrm{~s}$ with an overshoot of $0.02 \mathrm{pu}$.

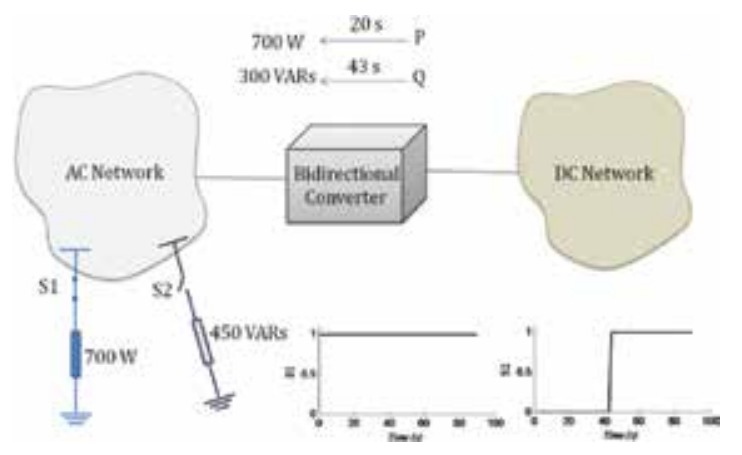

Figure 18. MGCC interaction with the main grid's controller.
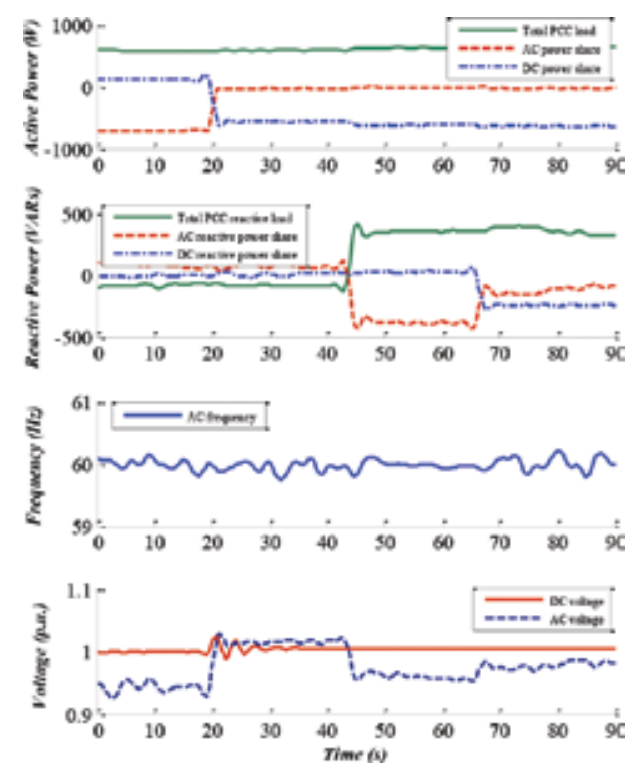

Figure 19. Response of the DC microgrid in an integrated hybrid AC/DC system corresponding to step changes in the load demand reference: (a) the load, DC, and AC active power share; (b) the load, DC, and AC reactive power share; (c) the frequency of the AC bus; and (d) the voltage of the AC and DC buses.

When a reactive load of $0.45 \mathrm{kVARs}$ with lagging power factor is added to the PCC after $43 \mathrm{~s}$, the voltage drops to around $0.95 \mathrm{pu}$. The DC microgrid is then requested by the main grid to 
inject $0.3 \mathrm{kVARs}$. Therefore, the voltage at the PCC increases to $0.98 \mathrm{pu}$. The DC bus voltage is hardly affected by this change in its reactive power reference. A maximum of $0.2 \mathrm{~Hz}$ frequency deviation was reported, as shown in Figure 19. This experiment highlights the coordination that can be achieved between the main grid's controller and the MGCC to enhance the overall performance of the whole system [18].

\section{Author details}

Ahmed Mohamed

Address all correspondence to: amohamed@ccny.cuny.edu

Smart Grid Laboratory, Department of Electrical Engineering, City College of the City University of New York, USA

\section{References}

[1] The White House, Washington. Presidential Policy Directive 21: Critical Infrastructure Security and Resilience (PPD-21) [Internet]. Feb. 2013. Available from: https:// www.whitehouse.gov/the-press-office/2013/02/12/presidential-policy-directivecritical-infrastructure-security-and-resil [Accessed: Feb. 2016].

[2] Olivares, D.E.; Mehrizi-Sani, A.; Etemadi, A.H.; Canizares, C.A.; Iravani, R.; Kazerani, M.; Hajimiragha, A.H.; Gomis-Bellmunt, O.; Saeedifard, M.; Palma-Behnke, R.; Jimenez-Estevez, G.A.; Hatziargyriou, N.D. Trends in microgrid control. IEEE Transactions on Smart Grid. 2014;5(4):1905-1919.

[3] Guerrero, J.M.; Vasquez, J.C.; Matas, J.; de Vicuña, L.G.; Castilla, M. Hierarchical control of droop- controlled AC and DC microgrids-A general approach toward synchronisation. IEEE Transactions on Industrial Electronics. 2011;58(1):158-172.

[4] Bidram, A.; Davoudi, A. Hierarchical structure of microgrids control system. IEEE Transactions on Smart Grid. 2012;3(4):1963-1976.

[5] Dragicevic, T.; Lu, X.; Vasquez, J.C.; Guerrero, J.M. DC microgrids-Part II: A review of power architectures, applications, and standardization issues. IEEE Transactions on Power Electronics. 2016;31(5):3528-3549.

[6] Elsayed, A.; Mohamed, A.; Mohammed, O. DC microgrids and distribution systems: An overview. Electric Power Systems Research. 2015;119:407-417.

[7] Balog, R.S.; Krein, P.T. Bus selection in multibus DC microgrids. IEEE Transactions on Power Electronics. 2011;26(3):860-867. 
[8] Majumder, R. A hybrid microgrid with DC connection at back to back converters. IEEE Transactions on Smart Grid. 2014;5(1):251-259.

[9] Nejabatkhah, F.; Li, Y.W. Overview of power management strategies of hybrid AC/DC microgrid. IEEE Transactions on Power Electronics. 2015;30(12):7072-7089.

[10] Yu, X.; She, X.; Zhou, X.; Huang, A.Q. Power management for DC microgrid enabled by solid-state transformer. IEEE Transactions on Smart Grid. 2014;5(2):954-965.

[11] Mohamed, A.; de Cossio, F.C.; Ma, T.; Farhadi, M.; Mohammed, O. Operation and protection of photovoltaic systems in hybrid AC/DC smart grids. IECON 2012-38th Annual Conference on IEEE Industrial Electronics Society. 2012, Montreal; 1104-1109.

[12] Li, W.; He, X. A family of isolated interleaved boost and buck converters with windingcross-coupled inductors. IEEE Transactions on Power Electronics. 2008;23(6):31643173.

[13] Elshaer, M.; Mohamed, A.; Mohammed, O. Grid connected DC distribution system for efficient integration of sustainable energy sources. IEEE/PES Power Systems Conference and Exposition (PSCE). 2011, Phoenix, AZ; 1-9.

[14] Mohamed, A.; Elshaer, M.; Mohammed, O. Bi-directional AC-DC/DC-AC converter for power sharing of hybrid AC/DC systems. 2011 IEEE Power and Energy Society General Meeting. 2011, San Diego, CA; 1-8.

[15] Dragicevic, T.; Guerrero, J.M.; Vasquez, J.C.; Skrlec, D. Supervisory control of an adaptive-droop regulated dc microgrid with battery management capability. IEEE Transactions on Power Electronics. 2014;29(2):695-706.

[16] Diaz, N.L.; Dragicevic, T.; Vasquez, J.C.; Guerrero, J.M. Intelligent distributed generation and storage units for dc microgrids - a new concept on cooperative control without communications beyond droop control. IEEE Transactions on Smart Grid. 2014;5(5): 2476-2485.

[17] Mohamed, A.; Salehi, V.; Mohammed, O. Reactive power compensation in hybrid AC/ DC networks for smart grid applications. 2012 3rd IEEE PES Innovative Smart Grid Technologies Europe (ISGT Europe). 2012, Berlin; 1-6.

[18] Salehi, V.; Mohamed, A.; Mazloomzadeh, A.; Mohammed, O. Laboratory-based smart power system, part II: Control, monitoring, and protection. IEEE Transactions on Smart Grid. 2012;3(3):1405-1417. 



\section{Section 2}

Development of Control Algorithms, Software Architecture and Simulation Tools for Energy Management Systems 



\title{
Development of an Energy Management System Control Algorithm for a Remote Community Microgrid System
}

\author{
Arno Vosloo and Atanda K. Raji \\ Additional information is available at the end of the chapter \\ http://dx.doi.org/10.5772/64034
}

\begin{abstract}
Rural communities are often unable to access electrical energy as they are located away from the national grid. Renewable energy sources (RESs) make it possible to provide electrical energy to these isolated areas. Sustainable generation is possible at a local level and is not dependent on connection to a national power grid.

The size of microgrid systems is dependent on the amount of energy that needs to be drawn and the amount of energy that has to be stored. Mechanical and electrical system component sizes become bigger due to increased operational energy requirements. Increases in component sizes are required on growing power networks when higher current levels are drawn.
\end{abstract}

Energy management of microgrids must thus be introduced to prevent overloading the power grid network and to extend the operational life of the storage batteries. Energy management systems (EMSs) consist of different components which are seen as operational units. Operational units are responsible for measurement, communication, decision-making and power supply switching control to manipulate the power output to meet the energy demands. Due to the increasing popularity of DC home appliances, it is important to explore the possibility of keeping these microgrids on a DC voltage basis. Electrical generation equipment such as photovoltaic panels can be used to generate DC at designed voltage levels. The energy management system connects the user loads and generation units together to form the microgrid.

Photovoltaic (PV) sources, energy management system (EMS) and user load parameters differ in the simulation software in order to observe how the control algorithm executes load shedding. A Stokvel-type charge share concept is dealt with where the state-ofcharge (SOC) of batteries and user consumption will determine how grid loads are managed. Load shedding within the grid is executed by monitoring energy flow and calculating how much energy is allowed to be used by each consumer. The energy management system is programmed to always provide the largest amount of energy to the consumer with the lowest energy consumption for each day. The batteries store surplus electrical energy during the day. Load shedding starts at 18:00 h each day. Users 
will be disconnected from the grid whenever their allocated energy capacity gets depleted.

Keywords: energy management system, remote microgrid, renewable, photovoltaic system, state of charge, logic control, Simulink, MATLAB

\section{Introduction}

Over 1.2 billion people around the world do not have access to electricity, including over 550 million people in Africa and 300 million people in India [1]. The traditional approach to serve these communities has been to extend the national grid to wherever possible.

This approach is technically and financially inefficient for remote communities due to a combination of capital scarcity, availability of insufficient energy, reduced grid reliability, extended building times and construction challenges to connect remote areas [1].

As the national grid is not accessible to all rural communities, power harnessing equipment must be brought to locations having the available electricity generation resources [2]. Currently, rural communities without access to electricity are dependent on natural resources such as wood and coal. Other processed resources such as liquefied petroleum gas, paraffin and candles are used for producing light and heat for their basic energy requirements.

Communities in rural Africa, though widespread, are quite isolated. Consequently, the energy suppliers find it difficult to supply electricity to these regions, as both capital and running costs for long-distance power transmission can be quite high. In addition, suppliers must provide suitably skilled personnel and deploy equipment from other areas, as rural villages do not have the required infrastructure or the skills required to install and maintain such infrastructure linking them to the national power grid. Hence, much of rural sub-Saharan Africa has limited or no supply of electrical energy from a national grid [3].

Basic electricity required for cooking and lighting, which are taken for granted in urban areas, is not readily available in remote rural areas. To resolve this problem, a constant supply of low-voltage power is required. The studies conducted so far have focussed only on the methods to supply electrical energy to these remote dwellings and also to control and manage the electricity being generated and consumed from microgrids [4]. Microgrids can be a better solution for these rural communities as they can provide the villages with their own electricity supply.

Microgrids are islanded systems that harvest, manage, distribute and consume electrical energy. A limited amount of energy can be stored by batteries. Various concept designs of microgrid technologies are being studied and practically implemented all over the world [5].

State of charge (SOC) is a measure of the full range of available energy that can be delivered by a battery. This range is measureable between when a battery is fully charged and when it is carries no or minimum residual charge according to the battery's electrochemical charac- 
teristics. Batteries are designed to store only a certain amount of energy. The design capacity of the battery determines the limited amount of energy that can be drawn from it. The available SOC of the batteries is distributed among the loads by the application of an energy management system (EMS).

Schnitzer et al. [1] recorded that technologically and operationally, microgrid distributed systems have the capacity to provide communities with electricity services, particularly in rural and peri-urban areas of less developed countries. Less developed countries do not have the national grid to feed power to rural settlements. Electrical energy can be generated with the latest microgrid technology at rural settlement locations.

By installing direct current (DC) microgrids, electrical energy can be harnessed from natural sources such as wind and solar radiation. Currently, in most countries, the national grids provide $110 \mathrm{~V}$ or $220 \mathrm{~V}$ alternating current (AC) for low-voltage applications required in households [6].

An AC must either be generated directly in its AC form, or alternatively it can also be converted to DC using an inverter system. The electronic components of inverters and rectifiers are expensive when considered for use in manipulating voltages in EMSs. When a DC grid is considered, DC generation is directly connected to the grid. Grid loads can be fed from this $\mathrm{DC}$ source and the need for converting AC to DC diminishes.

This study deals with the generation of DC, maintenance and management of a DC microgrid for the different agents and eradication of the expensive process involved in the conversion of AC to DC. Energy management of the grid is one of the key points to be observed in these microgrids for managing the loads and the storage units within the system.

\subsection{Objectives of the research}

This study aims at exploring an energy charge sharing strategy for an islanded DC microgrid. The system will be charged by solar energy. During the day, the grid will supply electrical energy to grid users. The software must be designed to simulate changes in the irradiance level of the sun. The irradiance adjustment can be used to simulate clouds and natural events that will diminish irradiance levels as the weather changes and also to simulate night-time, when there is no sun.

To record and store all the load user consumption figures and allow the system respond to the selected prescribed load shedding algorithms, function block logic is designed on the Matlab platform. It is envisaged that power measurement points will be installed on the bus system in order to provide load management functionality.

To determine the occurrence of load switching, sensing and control units act on command signals sent to and received from the energy management system (EMS). The system will add all the charge measurements received by the generation units. As load is applied to the respective load-points, the system will detect this load value, subtract it from the total charge load value and decide when each user will be disconnected from the system. Photovoltaic panels are used to generate electrical energy. The battery bank stores surplus electrical energy. 
The energy management system consists of measurement components and control components that act and execute functions as agents. The control philosophy will revolve around the agents to operate the charge share control objectives. Agents form a part of the system and work in coordination combining the overall system components. Environmental inputs, system control and execution of the software model environment will determine the microgrid load shedding procedure.

The physical location of the control agents should be determined by the system design. The power sizing per house unit per day needs to be estimated in order to obtain the total system power demand and the required system capacity. The system capacity needs to be calculated to determine the size of the batteries that will be included in the software model.

The software model should be accessible to change any parameter within the microgrid. It is vital to prepare a working platform of the microgrid on Simulink in order to support future research developments. The software model is designed to be flexible to allow user inputs to change the dynamics and control within the microgrid and to allow the grid to react to these adjusted parameters.

\section{Rural microgrid system layout}

Figure 1 shows a $13.5 \mathrm{~kW}$ photovoltaic system in Makueni County in Kenya [7]. This generation unit was designed and build to provide water and electricity to the local community. It must be noted that the DC-generating PV array shown in Figure 1 is located centrally in the village. Solar panels are used for conversion of solar energy into electrical energy. Microgrids seldom have long transmission lines to supply power to other towns and areas far from the point of generation.

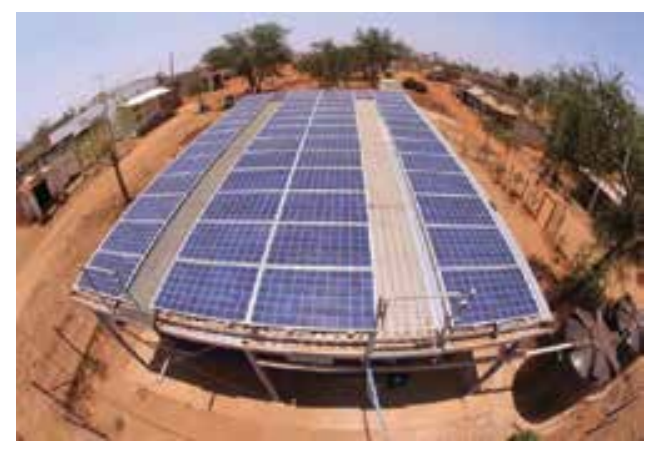

Figure 1. Makueni County 13.5 kW PV system, Kenya, Kitonyoni village market. (Phys. Org, 2013).

\subsection{Energy management systems and methods}

Testing of energy management systems in established microgrids is very limited because of the low number of such installations; however, Raji et al. has found that testing can be done 
with software-simulated models, using direct measurements collected from a few devices. Configurations in different geographical locations are not the same due to the huge differences in culture, climate and wealth of different regions. These differences determine the setting up of software simulators.

The EMSs of microgrids contain hardware and software protocols to form the operational platform of these control systems. Control and monitoring is carried out using an automated agent technology with a multi-agent system (MAS) [8]. Multi-agent systems take intelligent decisions on behalf of the user, instead of the more conventional supervisory control and data acquisition (SCADA), which is not flexible and requires regular monitoring and human intervention [8].

Load scheduling of MASs was presented by Logenthiran et al. [9]. Load scheduling is a means of optimizing microgrid operation efficiency by observing load recordings and paying particular attention to reducing the peak sections. Reducing peak load consumption during the day can result in an extended energy use during the night, as the surplus energy available when peak usage is reduced, And stored for use at night.

Different ways of integrating renewable resources with the community are available for microgrid layouts. Smart meters and smart stations form two essential parts whereby communication techniques share data in control architecture. Smart meters capture user power consumption and these figures are communicated to smart stations. Smart stations are linked to the aforementioned control architectures [10].

Jian et al. [11] presented a multi-agent-based control framework, stating that new types of power supplying modes have a promising future in the design of such frameworks. Their study demonstrated a software model segregated into three layers of operation; these layers include main grid, microgrid and component level agents. Coordination control represents a more efficient load control and charging due to the effective communication database among the three layers of operation.

Li et al. [12] state that these grids are attracting considerable attention owing to the increasing prevalence of DC home appliances and distributed energy resources. This study thus prompts us to consider a $48 \mathrm{~V}$ DC bus as the grid bus voltage level of choice.

Chaouachi et al. [13] explained that artificial intelligence techniques in cooperation with linear programming-based multi-objective optimization systems are being tested. Energy management in these systems aims to minimize the operational cost and the environmental impact of a microgrid. In order to manage future loads, these systems also take into account the load demands of operational variables.

In a different study by [14], Chaouachi et al. claimed that the results obtained from their control methods are also useful to extend battery charge and discharge cycles on a scheduled basis. These methods were obtained using software models and weather forecasting data. The results were used for comparing actual and predicted weather profiles several days ahead. These systems can hereby determine how load shedding can be controlled in future. Efficiency of these microgrid operations is strongly dependent on the battery scheduling process, which in 
turn is influenced by software systems programmed to anticipate weather conditions. Stluka et al. [15] stated that forecasting will mostly be based on a short-term forecasting concept. Stable cloud patterns lead to better predictions of future weather patterns, whereas unstable cloud formations make such predictions more difficult. Taking account of the predicted weather conditions, the system will consider the current state-of-charge of the storage units and determine the corresponding amount of energy to be allocated for consumption. The energy management system algorithm will reduce the distribution allowance to the loads and conserve energy if less charging is necessitated in future; this facilitates in extending the energy storage buffer of the system.

A different load-scheduled control strategy has been studied by Michaelson et al. [16]. Predicted generation is combined with predicted load. Past load patterns are available from load recordings made at the specific site. Automatic load shedding can be scheduled when the system can calculate during which future period the grid will be under severe strain. This would occur due to overloading or the unavailability of energy if the SOC of the batteries is too low. Future prediction of the SOC trajectory is achieved by this power management strategy.

Polycarpou [17] supports the idea that smart grids enable consumers to decide when they would use power from the grid. Microgrid philosophy is perhaps not a new concept, as it has been in use since the establishment of electricity grids. However, the control strategy is new and hence has been highlighted in this study. Modern control systems have resulted in smart microgrids to the extent wherein these grids can easily shift loads based on the differing needs and the desired outcomes.

As described by Ramesh et al. [18], Arduino-controller-based systems have been incorporated in actual microgrid installations; this type of system is described as an MAS. Arduino is a microcontroller that is programmed by an open-source software to fit the requirements of any programmer. Furthermore, many other types of microcontrollers are used to perform monitoring and control of microgrid systems. To build a central control system, external power modules are inserted into the I/O slots. The unit acts as a programmable logic controller (PLC) and can be expanded to control and manage systems accordingly.

Palma-Behnke et al. [19] explained that smart grids are characterized by a two-way flow of electricity and information. These smart grids are capable of monitoring every aspect from power plants to customer preferences as well as individual appliances. Real-time deliverable information is acquired and enables the near-instantaneous balance of supply and demand at the device level. Real-time information can operate at different intervals as long as it is located near the source of energy and the areas of supply delivery.

Meiqin et al. [20] have explained the three different levels of the EMS of a microgrid. These areas form a pyramid structure with linking layers between them. The top layer forms the local control level, including the communication control, and status agents. The SCADA platform is located at the central level. All the server agents and recording facilities are connected to the SCADA system. Level three is the system control level, wherein the system operator agents 
control the system. Grid status and switching between islanded mode and grid-connected mode is set here.

Boynuegri et al. [21] defined a load-shifting algorithm for home energy management (HEM) whereby the centre of control is dependent on the SOC of the batteries. By introducing these peak-shaving and load-shifting techniques, a $25 \%$ saving was evident from these simulation results. A study of battery capacity was conducted to compare battery sizing when batteries are put under charge and discharge conditions. Energy was also supplied to the main grid from this battery system. Microgrid battery systems should not be over- or undersized. Oversized battery systems do not get sufficient consumption drainage, and undersized systems deplete rapidly depending on the amount of energy drawn from the battery system. From an evaluation done by Yen-Haw et al. [22], it was shown that systems should be sized by battery efficiency and power supply generators.

Intelligent load management functions were studied by Kennedy et al. [23]. They focussed on approaching load management through a load-shedding scheme. They proposed an intelligent load shedder (ILS) principle that works together with a static transfer switch (STS). The STS operates as the PCC between the grid supply and the user loads. Loads are systematically removed from the grid to allow the grid sources to carry the remainder of loads.

According to Hajimohamadi et al. [24], proper system controllers in the primary, secondary and emergency levels of microgrids ensure stable operation during load shedding. These controllers prevent the system from breaking down to the level of a complete blackout; they assist in shedding load in a controlled manner. Similar to the conventional systems, isolated microgrids are also affected by different events such as tripping generators, imbalance between generation and consumption and power quality issues. All loads are not simultaneously disconnected; non-critical loads are disconnected first and then the software program will prepare for load shedding of power consumers.

From the above-mentioned studies and taking account of all the cited references, it is evident that the focus is on load control techniques and battery charge methods. These systems are linked together through various communication protocol methods that act as the nerve structure of the system. Due to the limited charge capacity that is available from the batteries at sunset, focus can be directed towards load-shedding control techniques around the SOC values of the batteries.

The conclusions from the above-mentioned studies serve as a motivation to incorporate Stokvel philosophy in the load control so as to develop a reserve share concept proposed by [25].

\section{Microgrid technology}

\subsection{Agent-based technology}

Agents are responsible for information flow within the system. The term 'agent' is characterized by the meaning of autonomy. [34] identified agent technology consisting of networks 
within a microgrid system of multi-agent modules or components which together form MASs. These networks of agents are used in the control of wind turbines and solar arrays. In addition, they are responsible for directing communication in the decision-making processes and executing actions.

Artificial intelligence (AI) is a research topic; MAS forms a subsection of distributed AI. The goal of an MAS is to disassemble a large complicated system that consists of software or hardware into multiple interactions and subsystems which can be easily managed. Microgrids are gradually providing a new method for developed countries to solve problems relating to the national grid. With the intensification of the global energy crisis, the microgrid will become an important development in this sector.

The United States first proposed the concept of a microgrid. In 2002, the Consortium for Electric Reliability Technology Solutions (CERTS) stepped forward to define the concept and structure of such a system [26].

The flow chart for the EMS considered in the paper is shown below.

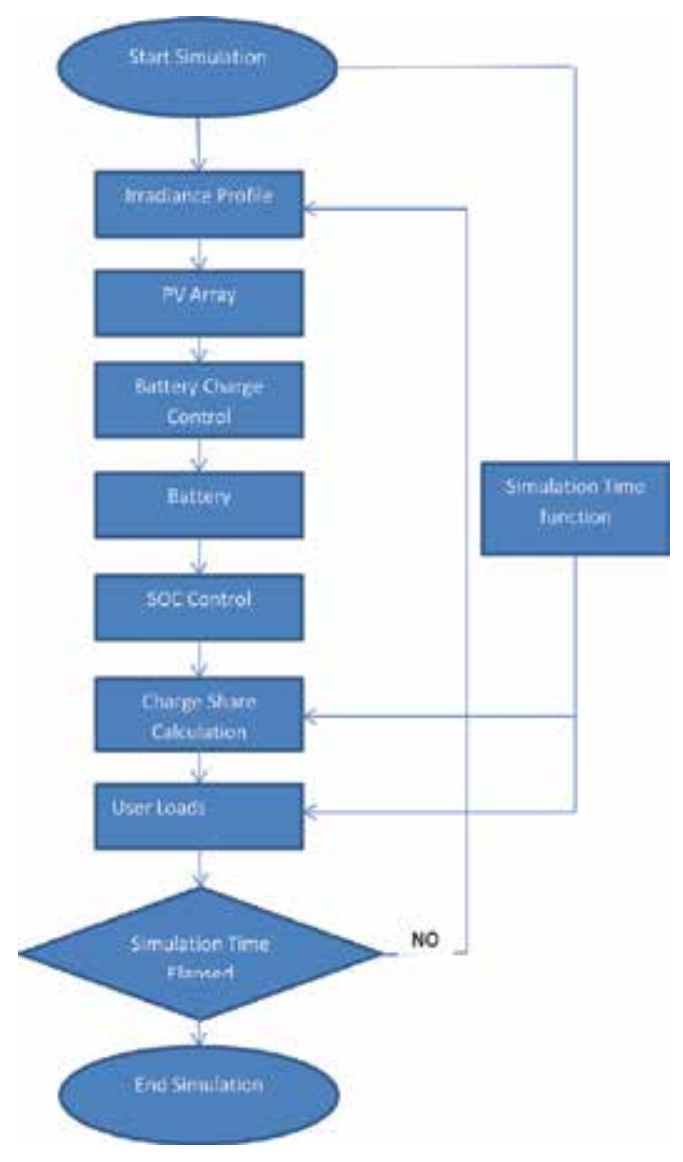




\subsection{Microgrid communications}

As microgrids expand, measurement points become more decentralized. Due to increased difficulty in capturing data over a larger distance, communication protocols are being heavily relied upon for assisting with this problem. Advanced communication technologies such as optical internet protocol (IP) networks, band-pass limiter (BPL) carrier and broadband wireless network technology are used [27]. It is very important to have as many measurement points as possible in order for the microgrid to provide flexibility in the supply of high quality. The uploading speed of data from monitoring should be high, in order to react to issues and to allow real-time control between the microgrid and its energy sources. Hernandez et al. [28] indicated that the smart distribution network requires periodic fast measurements and estimations of network security as well as real-time information from network components.

Xinhua et al. [27] presented three categories of communication namely knowledge interchange format, knowledge query and manipulation language and FIPA agent communication language. FIPA is a body for developing and setting computer software standards. In recent years, the extensible mark-up language (XML) has become an acceptable language for agent communication [29]. XML is a language used to describe data.

Multi-agent architectures are divided into two coordination categories: centralized multiagent coordination and de-centralized coordination.

\subsection{Centralized multi-agent coordination}

Figure 2 illustrates that coordinators are situated at the head of the architecture. They are responsible for communication between other agents. Within the architecture, the main representatives of agent coordinators are the mediator and the facilitator. The mediator agent makes decisions on lower-level situations. It is involved with message interpretation and task decomposition. A program that handles coordination between communication agents is called the facilitator. Based on their content, routing of messages is controlled and control of multiagent activities is regulated as well [30].

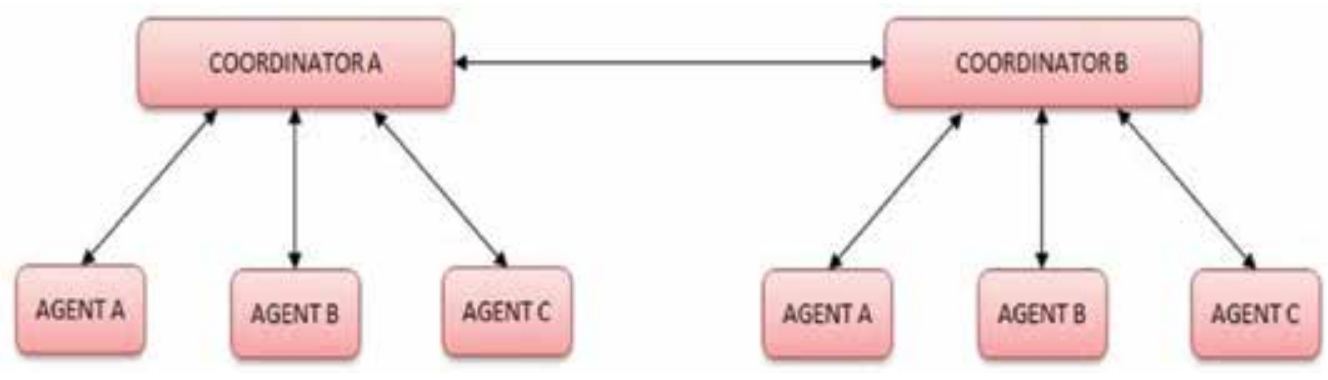

Figure 2. Centralized multi-agent coordination [29]. 


\subsection{De-centralized multi-agent coordination}

Figure 3 shows the de-decentralized coordination as an agent working in conjunction with linked agents; this type of coordination can be defined as autonomous. An agent operating in a de-centralized coordination setup is not controlled by software or a person; it communicates directly with other agents in the system by regularly scanning the status and tasks performed by other agents and executing its own tasks by associated motives. Communication is present between all the agents as shown in Figure 3. Communications to other networks are also possible by these agents.

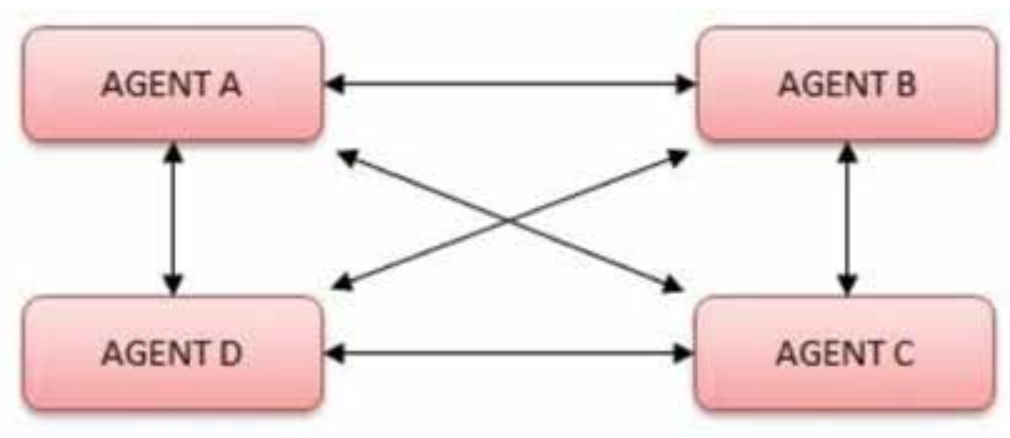

Figure 3. De-centralized multi-agent coordination [29].

\subsection{Control methods}

Considering continuity of supply to islanded microgrid loads and national grid loads, it is important that the power to loads is not interrupted during transitioning of the microgrid onto, or off the national grid. Developing a new interface technology is constantly undertaken to handle transitions between these modes. Currently, there are three typical control methods as explained by [27], namely plug-and-play technologies, power electronic devices and controlbased multi-agent technology. All three methods have progressed technologically to differing degrees, and it is understood that they will mature and grow with the application of sustained development in this field [27].

\subsection{Multi-agent modelling and implementation}

In order to build a complex agent system in a simplified manner, open-source platforms are used by developers. Hernandez et al. used the JADE platform for their study.

JADE facilitates the development of multi-agent peer-to-peer applications. Parameters of this system aim at the optimal use of locally distributed resources, feeding of local loads and operational simplicity. Direct support of plug-and-play can be used by the JADE-based platform.

Jimeno et al. [31] referred to a demand profile of the grid. This is the map and baseline prediction load graph that is used for the load reference. The profile indicates the power 
demand (in $\mathrm{kW}$ ) versus time of the day. In coupled microgrids, it is vital to use this baseline as a reference when energy transfer and power flow are to be controlled. The demand profile was used as a reference to control how and when the grid sources should be adjusted to the load. Costs to run the individual source units were also monitored as load demands changed. The system makes decisions around cost and executes accordingly in order to use the most affordable option to generate energy; the system makes adjustments where needed.

\subsection{Agents in the energy management systems}

Schedule trackers are used to allocate set points to distributed energy resource (DER) gateways. Schedules are calculated by observing the recorded data. The microgrid operator agent is used to optimize the operation of the microgrid by taking into account the information provided by the agents in the local controllers. DER operator agents are used to provide information to the microgrid level operator and also to act accordingly while receiving signals from it.

\section{System design for rural settlement}

The design of a three-house islanded microgrid is used in this study to serve as an example of the charge share concept. When a small microgrid system has been successfully designed, a multiplication factor can be applied to allow for an increase in the number of houses and the corresponding increase in total load that would be required for such a macroconsumer design. Solar irradiance profiles for the Western Cape, South Africa, have been dealt by [30].

Software development packages such as Matlab are available into which the grid design parameters can be inserted. These design packages then calculate the number of solar panels to be used and the size of each unit and also determine the battery size. The designer can also enter weather and day-time parameters to monitor the microgrid as a whole.

\subsection{User load demand}

The case study example given below provides the times and load averages used to determine the system generation units. First, the size $(\mathrm{kW})$ and duration $(\mathrm{h})$ of load consumption need to be established before the system generation units can be designed.

Example: A rural house is assumed to have an average load of $1 \mathrm{~kW}$. The duration of load consumption is set to $3 \mathrm{~h}$ (between 05:00 and 08:00) in the morning, $2 \mathrm{~h}$ (between 12:00 and 14:00) during lunchtime and $5 \mathrm{~h}$ (between 18:00 and 23:00) in the night.

\subsection{Battery sizing}

To determine the battery size for a house unit, a few design specifications need to be confirmed before calculations are done. Daily energy demand needs to be considered. The duration of load supply per day and the number of days the system would be capable to provide energy 
to the load must be determined; this is known as the autonomy of the system. Based on the example mentioned above, an average power consumption of $1 \mathrm{~kW}$ sustained for $10 \mathrm{~h}$, yields an average daily energy requirement of $10 \mathrm{~kW} \mathrm{~h}$.

The average daily energy requirement must be multiplied by a factor of 1.2-1.5 to allow for power losses due to increases in temperature and spike [32]. This factor also allows for decreasing performance when the temperature increases. Multiplying the required $10 \mathrm{~kW} \mathrm{~h}$ by the 1.5 loss factor will give a required total battery energy rating of $15 \mathrm{~kW} \mathrm{~h}$ for 1 day.

\subsection{Autonomy of the system}

It must also be decided how many days' worth of energy must be stored in the battery bank. Generally, system designs allow for an autonomy range of 2-5 days. In the case of the example, an autonomy capacity of 2 days will be taken into account. The total battery energy of $15 \mathrm{~kW}$ $\mathrm{h}$ is multiplied by two and results in $30 \mathrm{~kW} \mathrm{~h}$, which then gives system autonomy of 2 days.

\subsection{Battery bank capacity}

Lastly, the minimum battery capacity (in ampere-hours) must be calculated. To prevent an SOC below $50 \%$ for the batteries, the total battery energy should be double the required energy rating inclusive of the loss factor and the system autonomy. By multiplying the $30 \mathrm{~kW}$ h by two to give $60 \mathrm{~kW} \mathrm{~h}$, the total battery energy capacity requirements were determined.

The capacity rating of a battery was determined by dividing its energy output by the battery voltage. A voltage of $48 \mathrm{~V} \mathrm{DC}$ was chosen for the microgrid; hence, $48 \mathrm{~V}$ batteries are required. Therefore, a total battery energy rating of $60 \mathrm{~kW}$ h will have a required capacity of $1250 \mathrm{~A} \mathrm{~h}$. This battery capacity will be sufficient to enable a fully loaded battery to run expected loads for 2 days, at $10 \mathrm{~h}$ a day, without recharging. For a 1-day system autonomy, battery capacity of $625 \mathrm{~A} h$ is required.

Eq. (1) is an expression used to formulate the battery capacity.

$$
\text { Battery } \operatorname{Capacity}(\mathrm{Ah})=\frac{(\text { Average daily energy requirement }) \times(\text { Days of autonomy }) \times 2 \times 1.5}{(\text { Nominal battery voltage })}
$$

\subsection{Sizing PV panels to meet system battery requirements}

This section describes how PV panels are chosen for a system to meet the battery requirements. System designs can vary with panel voltage ratings. Common voltages to choose from are 12 $\mathrm{V}, 24 \mathrm{~V}$ and $48 \mathrm{~V}$. These panels can be configured such that the optimum design is achieved.

A solar PV panel with specifications as shown in Table 1 was chosen for this investigation. Maximum power voltage indicates that this panel will provide $47.5 \mathrm{~V}$, which is suitable for a $48 \mathrm{~V}$ microgrid bus system. 


\begin{tabular}{ll}
\hline Module specification & Description \\
\hline Model number & SM 350(40) P 1946 × 1315 \\
Module type & Poly \\
Maximum power $\left(\mathrm{P}_{\max }\right)$ & $350 \mathrm{~W}$ \\
Open circuit voltage $\left(\mathrm{V}_{\mathrm{oc}}\right)$ & $59.37 \mathrm{~V}$ \\
Short circuit current $(\mathrm{Isc})$ & $7.96 \mathrm{~A}$ \\
Maximum voltage $\left(\mathrm{V}_{\mathrm{m}}\right)$ & $47.5 \mathrm{~V}$ \\
Maximum current $\left(\mathrm{I}_{\mathrm{m}}\right)$ & $7.37 \mathrm{~A}$ \\
Maximum system voltage & $1000 \mathrm{~V}$ DC \\
Operating temperature & $-40{ }^{\circ} \mathrm{C}$ to $85^{\circ} \mathrm{C}$ \\
Module size (mm) & $1946 \times 1315 \times 50 \mathrm{~mm}$ \\
Mass & $35 \mathrm{~kg}$ \\
Material & Polycrystalline silicon \\
Number of cells & 96 \\
\hline
\end{tabular}

All technical data at standard test condition: Air mass unit $=1.5$, Irradiation $=1000 \mathrm{~W} / \mathrm{m}^{2}$, Cell temperature $=25^{\circ} \mathrm{C}$.

Table 1. Solar panels, $48 \mathrm{~V}, 350 \mathrm{~W}$ - specification (adapted from Solare 2015).

Figure 4 shows the average daily sunlight and daylight hours in Cape Town for each month of the year.

\section{Sunshine \& Daylight Hours in Cape Town, South Africa}

- Mean sunshine hours in Cape Town range from 5:42 each day in July to 11:05 each day in December

- The longest day of the year is $14: 15$ long and the shortest day is $9: 44$ long.

- The longest day is $4: 31$ longer than the shortest day.

- There are an average of 2993 hours of sunlight per year (of a possible 4383) with an average of $8: 11$ of sunllight per day.

- It is sunny $68.3 \%$ of daylight hours. The remaining $31.7 \%$ of daylight hours are likely cloudy or with shade, haze or low sun intensity.

Average Sunlight Hours/

Day

Jul Aug Sep Oct Nov Dec lan Feb Mar Apr May Jun Annual

Average Daylight Hours \&

Minutes/ Day

Percentage of Sunny

(Cloudy) Daylight Hours

05:42 06:25 07:12 08:54 09:54 11:05 10:54 10:24 09:05 06:54 05:54 06:00 08:11

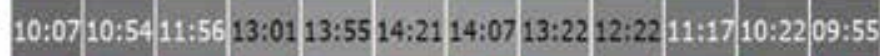

Figure 4. Sunshine \& daylight hours in Cape Town [33]. 
To estimate the size of the solar array, the system power requirements must first be determined, followed by the number of hours per day the system will be required to supply and meet the load demand. Many guidelines in the industry enable the PV designer to plan and build such a system.

The following example explains the method used for sizing the PV panels of a system for 1day autonomy on 1 house unit.

In December, during the summer months, the average daily sunlight is $11.1 \mathrm{~h}$, while the shortest daily average of $5.7 \mathrm{~h}$ occurs in July. First, the daily battery capacity requirements are taken from Section 4.5. In this case, the $625 \mathrm{~A} \mathrm{~h}$ unit is considered. Then the sun hours that are available for a day must be determined. Note that the month with the shortest average daily sunshine hours be considered; this will ensure that the system will receive an adequate charge in winter.

To obtain the total current rating required from the PV array, the 1-day autonomy $625 \mathrm{~A} \mathrm{~h}$ value is divided by the shortest average daily sunlight which gives $110 \mathrm{~A}$.

Next the number of solar panels required needs to be calculated. Take the current rating required from the array and divide it by the current rating of the panel. The result is 14.7 units and is rounded up to 15 panels.

The 15 PV units will be connected in parallel so that the total current will be 110, 6 A under peak conditions. The 15 panels as calculated meet the requirements of one house; for three houses and array of 45 panels all in parallel would therefore be needed.

\section{MAS algorithm development}

To describe the different aspects within the system as well as functionality, the Matlab software model was developed in subcomponents. The different function blocks fit together in order to merge the different area types. The model is divided into physical power circuits and signal circuits.

Power circuits form part of the generation and delivery of electric power to the storage batteries and grid loads. These types of power circuits also include the switching devices, load components as well as the photovoltaic generation blocks.

The second programming block consists of lines identified as part of the signals and does not represent physical lines. These circuits function in response to the result or output of the calculations. Programming blocks include calculation blocks that consist of analog and digital signals. The logic is combined to simulate and execute the algorithms as shown in Figure 5.

\subsection{Scenario A}

The first model, Scenario A, represents a basic user profile. The irradiance boost constant was selected as 45 to simulate the incorporation of $45 \mathrm{PV}$ panels for grid generation. An SOC of 


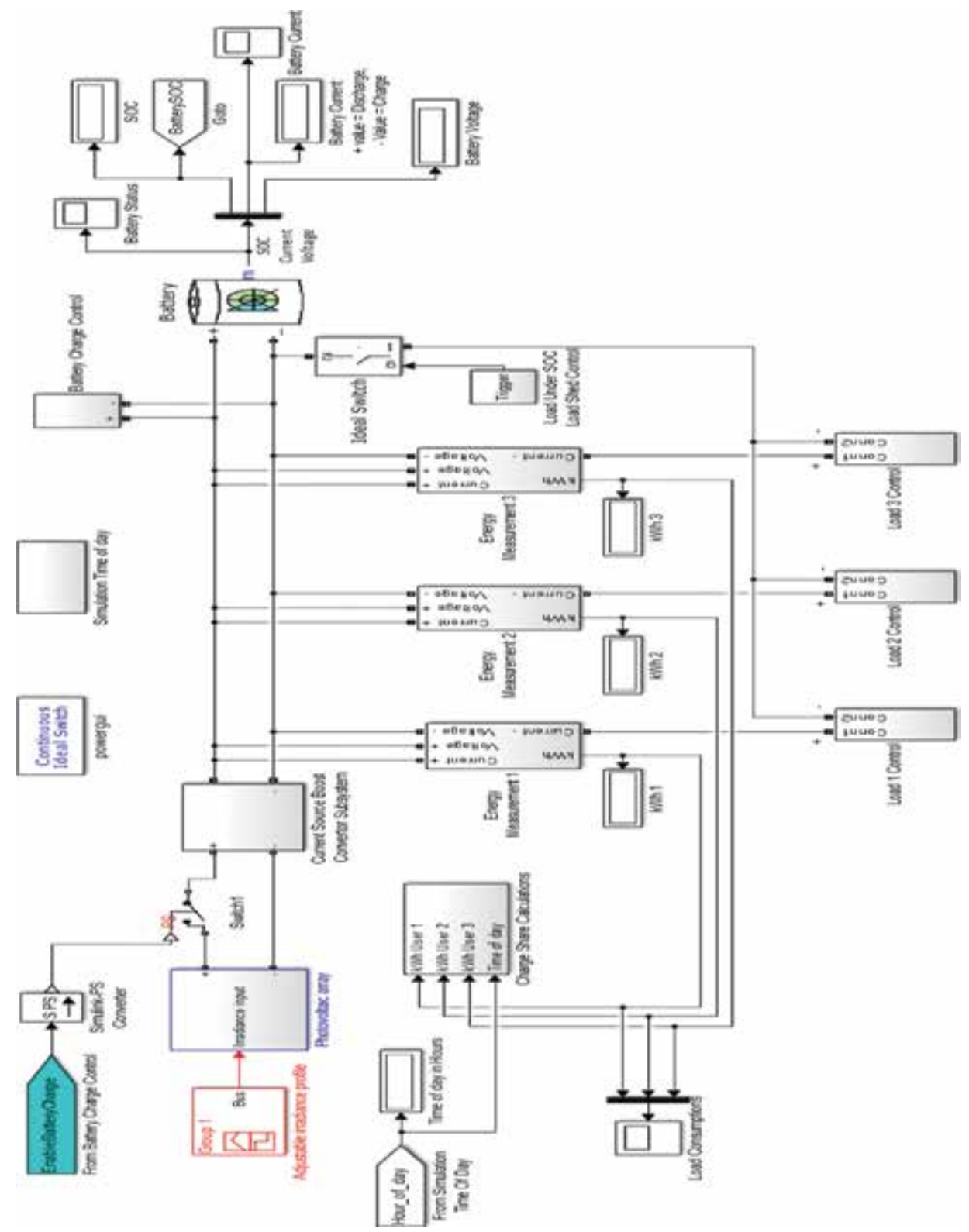

Figure 5. Matlab model overview.

$100 \%$, representing a fully charged battery, was selected, while a battery capacity rating of 2000 $\mathrm{A} \mathrm{h}$ was chosen. To validate that the system mathematics are functional, the loads and durations were made the same for all three users. Each user used $1000 \mathrm{~W}$ continuous power for a full day. The expected result should reflect equal energy division between all users. Table 2 indicates the initial parameters for setup to Scenario A. 


\begin{tabular}{ll}
\hline Irradiance profile & See Figure 6 \\
\hline Irradiance boost constant & 45 \\
Battery size and capacity & Voltage: $48 \mathrm{~V} \mathrm{DC}$ \\
& Capacity: $2000 \mathrm{~A} \mathrm{~h}$ \\
& Initial SOC: $100 \%$ \\
User 1 load-time reference & Load size: $1000 \mathrm{~W}$ \\
& Consumer demand times: $24 \mathrm{~h}$ \\
User 2 load-time reference & Load size: $1000 \mathrm{~W}$ \\
& Consumer demand times: $24 \mathrm{~h}$ \\
User 3 load-time reference & Load size: $1000 \mathrm{~W}$ \\
& Consumer demand times: $24 \mathrm{~h}$ \\
Load under SOC load shed control & SOC limit to reset and open disconnect all loads from the grid: $20 \%$ \\
& SOC limit to set and connect loads back to the grid: $60 \%$ \\
Battery charge control limits & Charge on: $\mathrm{V}_{\text {battery }}<8 \mathrm{~V}$ and $\mathrm{SOC}<0 \%$ \\
& Charge off: $\mathrm{V}_{\text {battery }}>5 \mathrm{~V}$ \\
\hline
\end{tabular}

Table 2. Scenario A: model setup parameters.

\subsection{Scenario B}

In Table 3, the setup for Scenario B is shown. The irradiance constant was set at 30 units to have a smaller charge effect on the grid compared to the case for Scenario A. The initial SOC was set at $65 \%$ to monitor the discharge characteristics of the DC bus. Variable loads were introduced for User 1, while the load User 2 and User 3 was, respectively, set to a constant 1200 $\mathrm{W}$ and $1000 \mathrm{~W}$. The aim was to inspect the control properties of the charge share calculations when the algorithm was introduced to variable load changes.

\begin{tabular}{ll}
\hline Irradiance profile & See Figure 6 \\
\hline Irradiance boost constant & 30 \\
Battery size and capacity & Voltage: $48 \mathrm{~V} \mathrm{DC}$ \\
& Capacity: $2000 \mathrm{~A} \mathrm{~h}$ \\
& Initial SOC: $65 \%$ \\
& Load size: $500 \mathrm{~W}+800 \mathrm{~W}$ \\
User 1 load-time reference & Consumer demand times for $800 \mathrm{~W}: 04: 00-07: 00$ \\
& $12: 00-13: 00$ \\
& $17: 00-23: 00$ \\
& Constant $500 \mathrm{~W}$ load for $24 \mathrm{~h}$ \\
& Load size: $1200 \mathrm{~W}$ \\
User 2 load-time reference & Consumer demand times: Morning: None \\
& Afternoon: None \\
& $18: 00-24: 00$
\end{tabular}




\begin{tabular}{ll}
\hline Irradiance profile & See Figure 6 \\
\hline User 3 load-time reference & Load size: $1000 \mathrm{~W}$ \\
& Consumer demand times: 04:00-07:00 \\
& 17:00-23:00 \\
Load under SOC load shed control & SOC limit to reset and open disconnect all loads from the grid: $20 \%$ \\
& SOC limit to set and connect loads back to the grid: $60 \%$ \\
Battery charge control limits & Charge on: $\mathrm{V}_{\text {battery }}<8 \mathrm{~V}$ and SOC $<0 \%$ \\
& Charge off: $\mathrm{V}_{\text {battery }}>5 \mathrm{~V}$ \\
\hline
\end{tabular}

Table 3. Scenario B: model setup parameters.

\subsection{Results and discussion of the graphs}

This section contains all the graphs and data recordings taken from the Matlab model scope measuring blocks.

\subsubsection{Scenario A: results and discussion}

The input setup prior to the simulation run for Scenario A was given earlier. Figure 6 shows that the battery SOC was $100 \%$ at the start of the simulation. The user loads were set up to be the same for this test and drew a combined total current of $60 \mathrm{~A}$. The battery voltage started at $56 \mathrm{~V}$ and reduced by $2 \mathrm{~V}$ when the load started to consume battery energy. For this scenario, the user loads were set up to draw power continuously throughout the 24 - $\mathrm{h}$ period of the

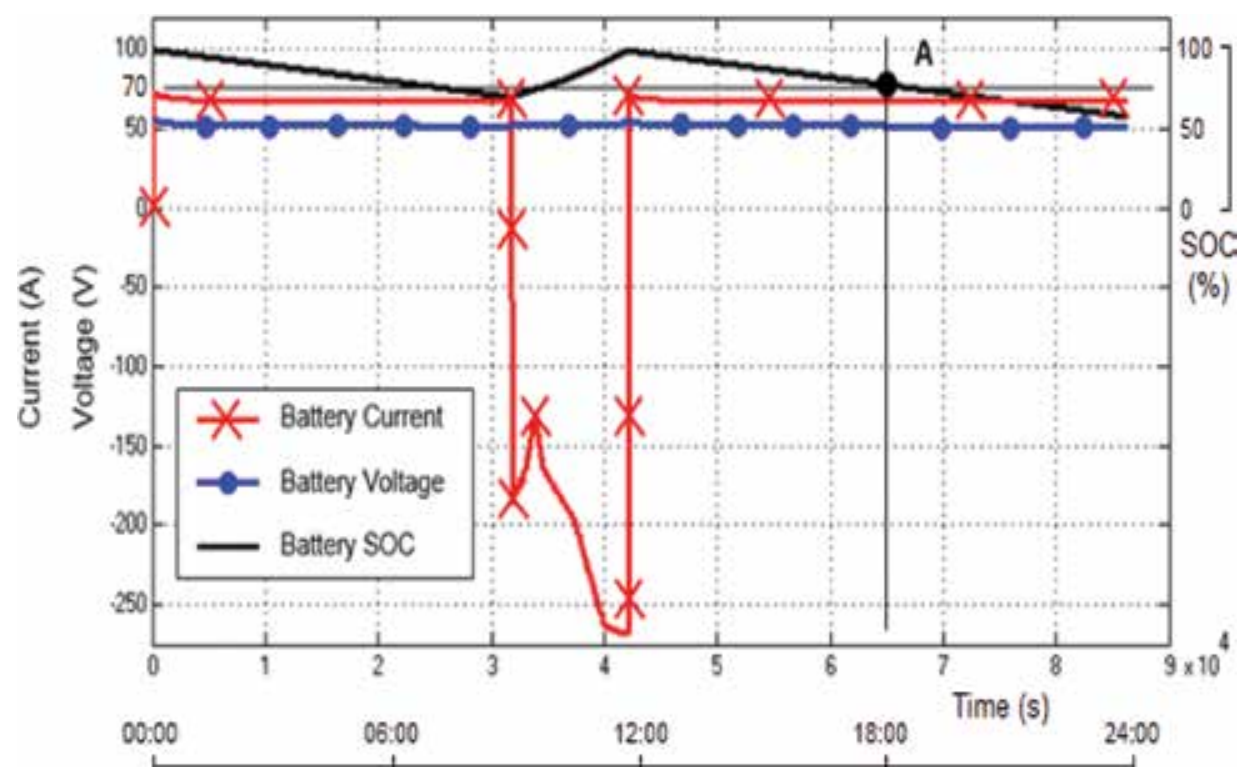

Figure 6. Scenario A: battery status. 
simulation. A gradual decline in the SOC was noted from 00:00 h until 08:48 h when SOC reached the $70 \%$ limit for charging. The charging parameters were set up to start charging at $70 \%$. Battery current dropped to negative $180 \mathrm{~A}$ when battery charging was initiated. The SOC increased to $100 \%$ from $08: 48$ to $11: 54$ due to charging. The battery voltage increased back to $55 \mathrm{~V}$ when the battery had been charged to $100 \%$.

Battery charging stopped at the 100\% SOC level at 12:00 h. Figure 6 shows that the user load consumption increased while charging stopped, due to the rise in the bus voltage when the battery SOC reached 100\%. As shown in Figure 6, the battery SOC decreased gradually after 12:00 h; a 2-3 V drop is noted when the SOC is decreased due to user energy consumption. Point A represents the point when the reserve share logic is activated in the simulation model. Without solar radiation, charging is not possible after 18:00 h; load control is activated beyond this time. Point B in Figure 6 shows all the load consumptions for the day at 18:00 h. All user loads were confirmed to have consumed the same power with all three consumption graphs tracking on top of each other. Point B also indicates that all users had consumed $21 \mathrm{~kW} \mathrm{~h}$ from 00:00 h until 18:00 h. Charge reserve share energy was continuously calculated and reflected as shown in Figure 7.

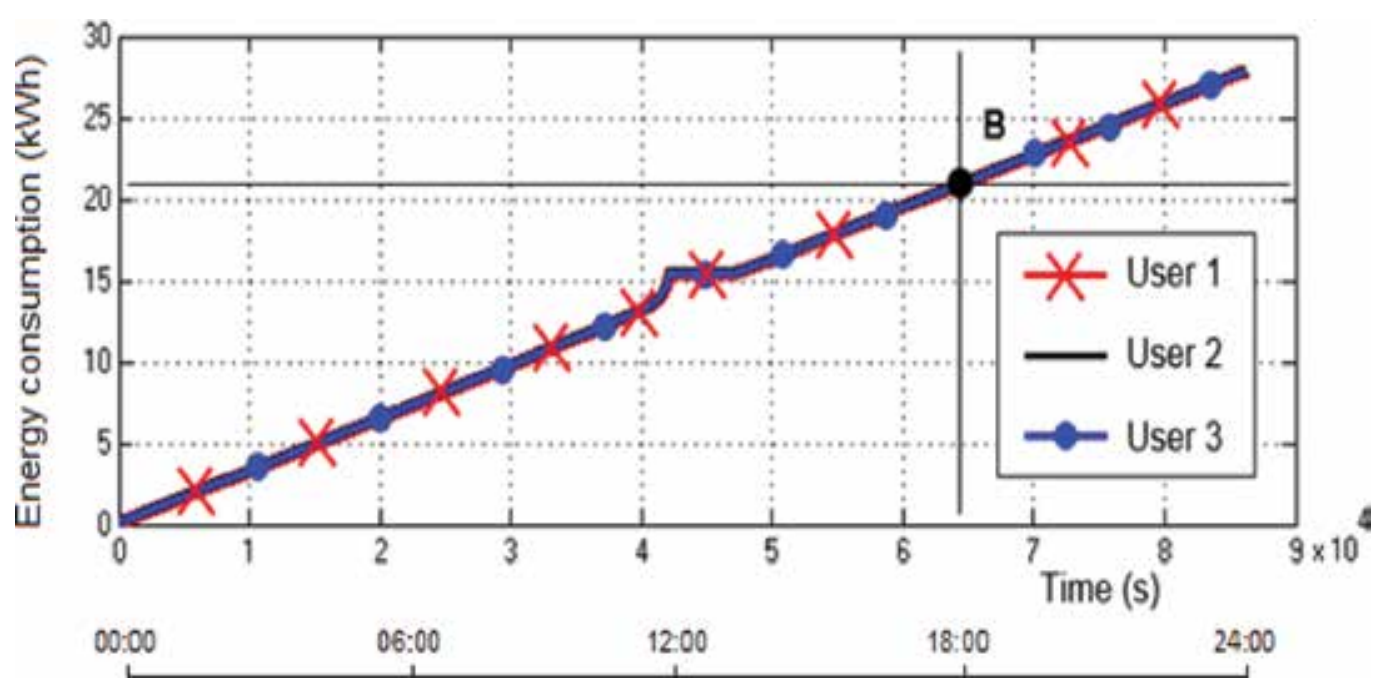

Figure 7. Scenario A: energy consumption.

The procedure described above was used to calculate the percentages of electrical energy that were allowed at the given battery SOC. The full battery at start-up time, 00:00, held $76 \mathrm{~kW} \mathrm{~h}$ of usable energy which represented $80 \%$ of its SOC. Each user was allocated a third of this starting total, which was $25.3 \mathrm{~kW}$ h. The energy values that were allocated to each user were the same because the energy consumption of each user during the day from 00:00 $\mathrm{h}$ to 18:00 $\mathrm{h}$ was identical; hence the trend lines as shown in Figure 7 run on top of each other.

If the change in energy allocation in Figure 7 is compared to the battery SOC in Figure 6, it will be observed that the change in allocation of energy to each of the three users was directly 
related to the change in battery SOC. Point $\mathrm{C}$ at 18:00 h in Figure 8 shows the point at which the reserve share logic was activated.

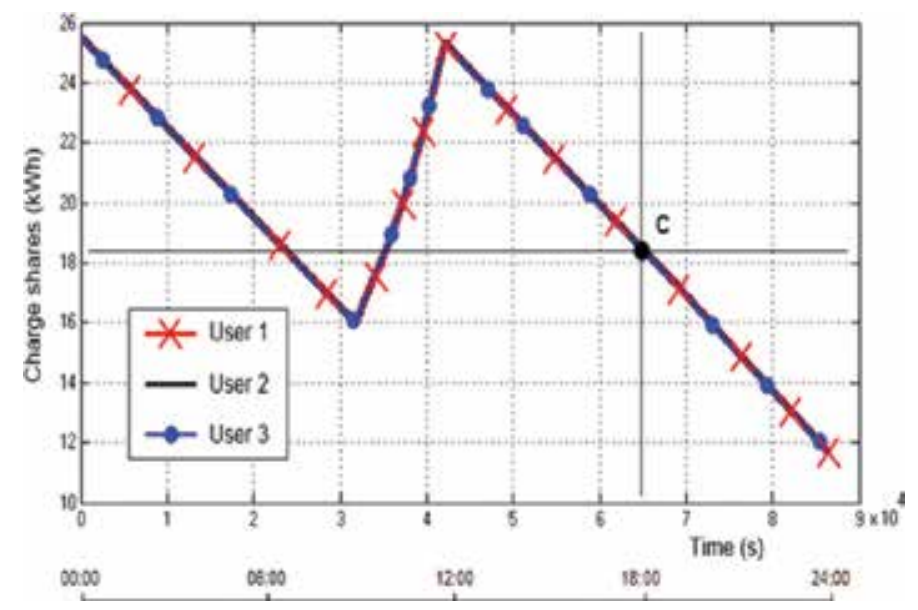

Figure 8. Scenario A: energy allocation.

As shown in Figure 8, each user unit was allowed a $19.3 \mathrm{~kW} \mathrm{~h}$ energy portion from the remaining battery SOC at 18:00 $\mathrm{h}$.

Usable battery energy of $76 \mathrm{~kW}$ h is calculated where $20 \%$ SOC was subtracted from the full battery range of $96 \mathrm{~kW} \mathrm{~h}$. The algorithm divided the reserve share energy, equally between the three users, each receiving a 33.3\% energy share. This test confirmed that the reserve share logic and formula is functional with equal results of charge share when equal load consumption values were presented. Table 4 summarises the results of scenario A.

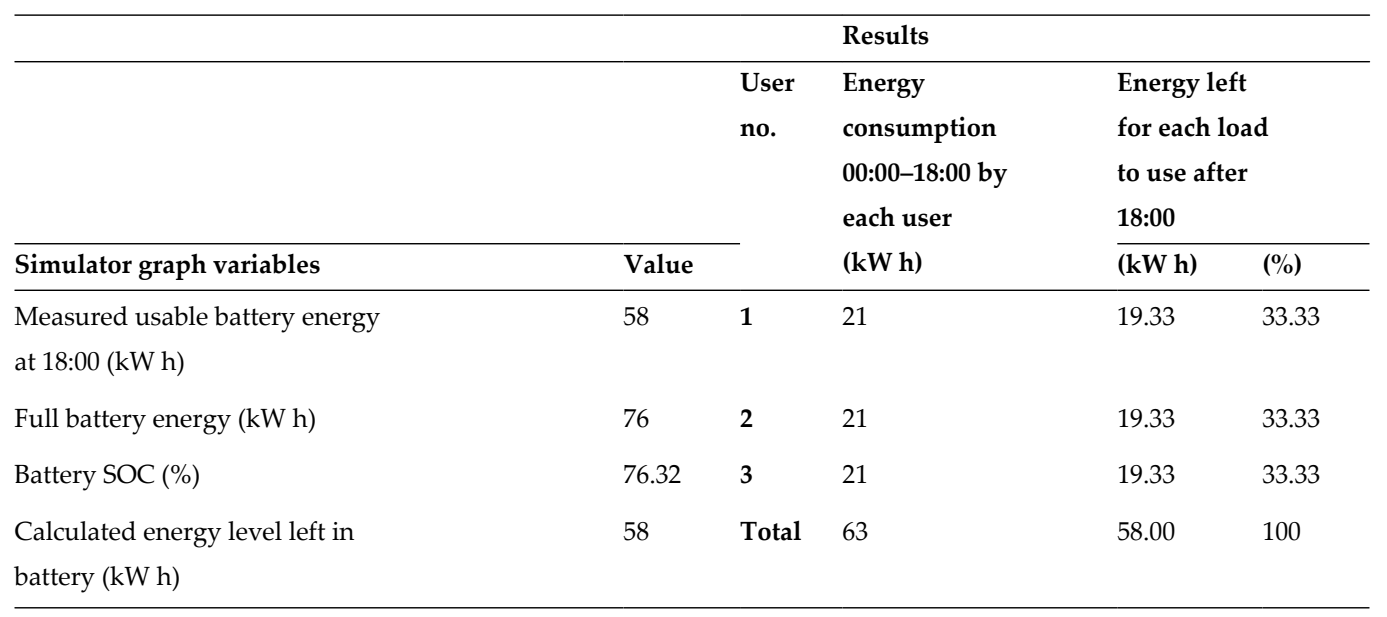

Table 4. Scenario A: result summary. 


\subsubsection{Scenario B: results and discussion}

In Scenario B, variable load changes were introduced to the system and the energy consumption changed to accommodate as described below. The following parameters have been applied to this set up:

- The SOC limit protection was set to disconnect user loads on $20 \%$ and enable load control on $60 \%$.

- The battery charge control limits were set at $48 \mathrm{~V}$ and $55 \mathrm{~V}$.

- Charging of the battery was set to trigger when the SOC dropped to $70 \%$.

As shown in Figure 9, the battery SOC starts to perform on the preset value of $65 \%$ and reaches $100 \%$ at $12: 00 \mathrm{H}$. When charging starts at $04: 15 \mathrm{~h}$, the current declines from a value of $+50 \mathrm{~A}$ to a value of $-220 \mathrm{~A}$ at 12:00 $\mathrm{h}$ when charging stops. Positive battery current spikes (in Figure 9) at 04:15 h, 12:00 h, 17:00 h and 18:00 h shows where user loads were applied to the system. Negative battery current shows that the system was charging from 04:15 $\mathrm{h}$ to 12:00 $\mathrm{h}$. The negative spikes at 13:00 $\mathrm{h}$ and 23:00 $\mathrm{h}$ indicate where user loads have been disconnected from the system. The negative spike at 21:30 h indicates where User 1 was disconnected when the user's remaining energy portion was depleted before the end of the day.

At Point A in Figure 9, the battery SOC is $90 \%$ at 18:00 h. Points B, D and C in Figure 10 show the respective cumulative energy consumption for each user at this time. User 1 has two constant loads, $500 \mathrm{~W}$ and $800 \mathrm{~W}$, respectively. The $800 \mathrm{~W}$ load is demanded intermittently and the $500 \mathrm{~W}$ load is required for the full 24-h period. User 2 starts to consume energy at 18:00 $\mathrm{h}$. User 3 consumed energy once in the morning at 04:00 $\mathrm{h}$ and then again at 18:00 $\mathrm{h}$.

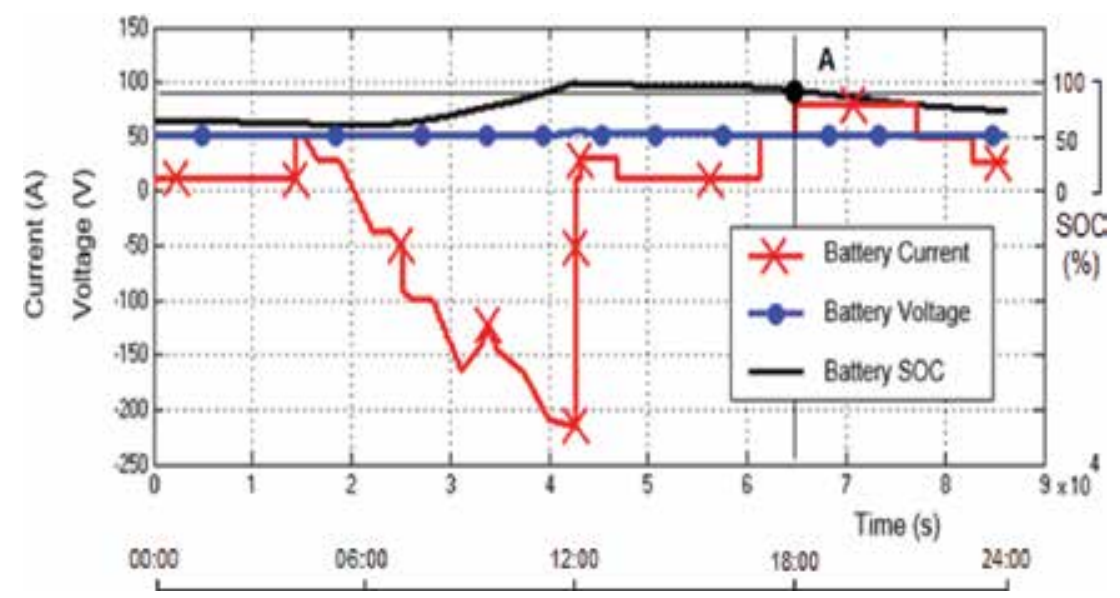

Figure 9. Scenario B: battery status. 


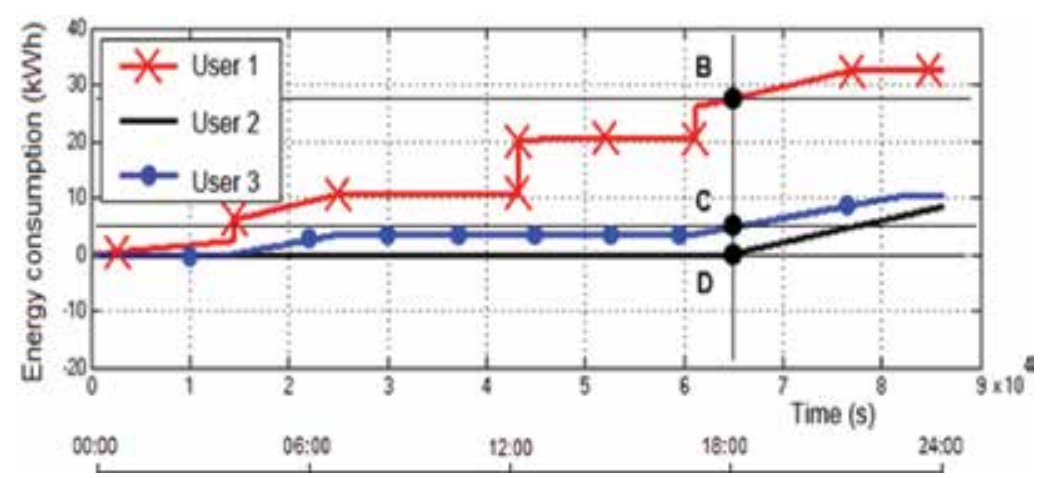

Figure 10. Scenario B: energy consumption.

Figure 11 indicates the charge values calculated throughout the day. As shown in Figure 10, User 1 at Point B was the biggest energy user. If we look at the red line that indicates User 1 in Figure 11, then it is evident that the charge share algorithm allowed the least amount of stored energy to this user. In the case of Users 2 and 3, the same amount of charge was allowed up to $04: 15 \mathrm{~h}$.

Points G, E and F show the respective charge share values for Users 1, 2 and 3 at 18:00 h. At 18:00 $\mathrm{h}$, the charge share algorithm stopped calculating the charge energy portions and only allowed the remaining portion energies to be deducted. This may explain why all the trend lines show a declining slope from 18:00 h onwards.

The lines show decreasing charge stress values when the loads are still consuming energy after 18:00 $\mathrm{h}$ and will change to horizontal lines when the loads are switched off inside the houses.

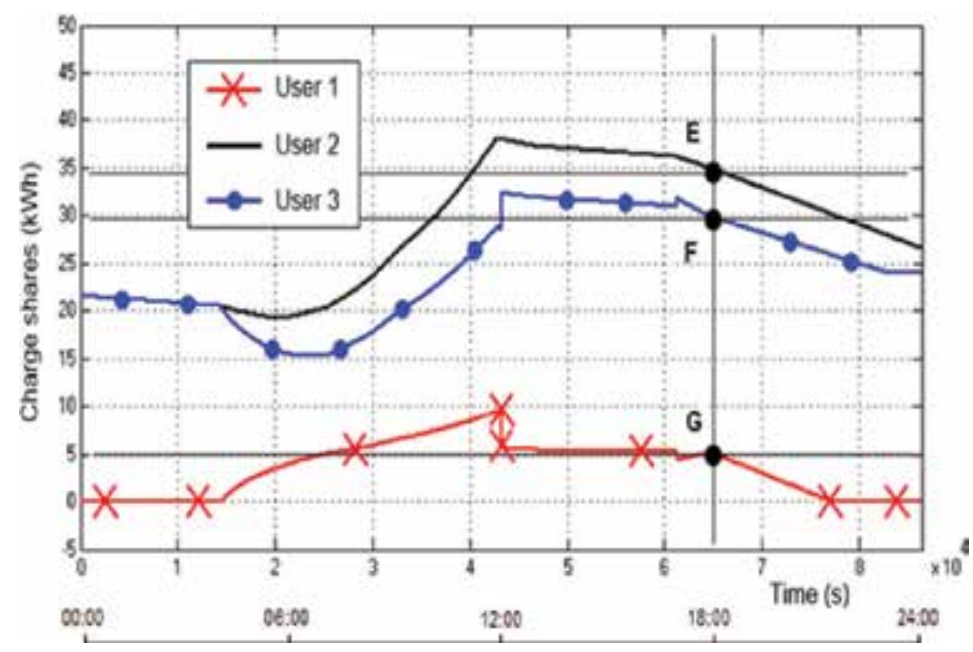

Figure 11. Scenario B: charge share. 
It is positively identified that User 1 used the most energy during the day and was allowed the smallest portion of battery SOC at 18:00 h. User 2 was allowed $50 \%$ of the remaining charge because no power was consumed during the day. User 3 received $42.42 \%$ of the remaining SOC and was recorded as the medium user for the day with a consumption of $5 \mathrm{~kW}$ h before 18:00 h.

This test proves the functionality of the charge share algorithm with variable load profiles operating on the microgrid. The biggest user of the day was allowed the smallest portion of remaining SOC energy at night, whereas the smallest power user was allowed the biggest share of available energy at night. Table 5 summarises the results of scenarion B.

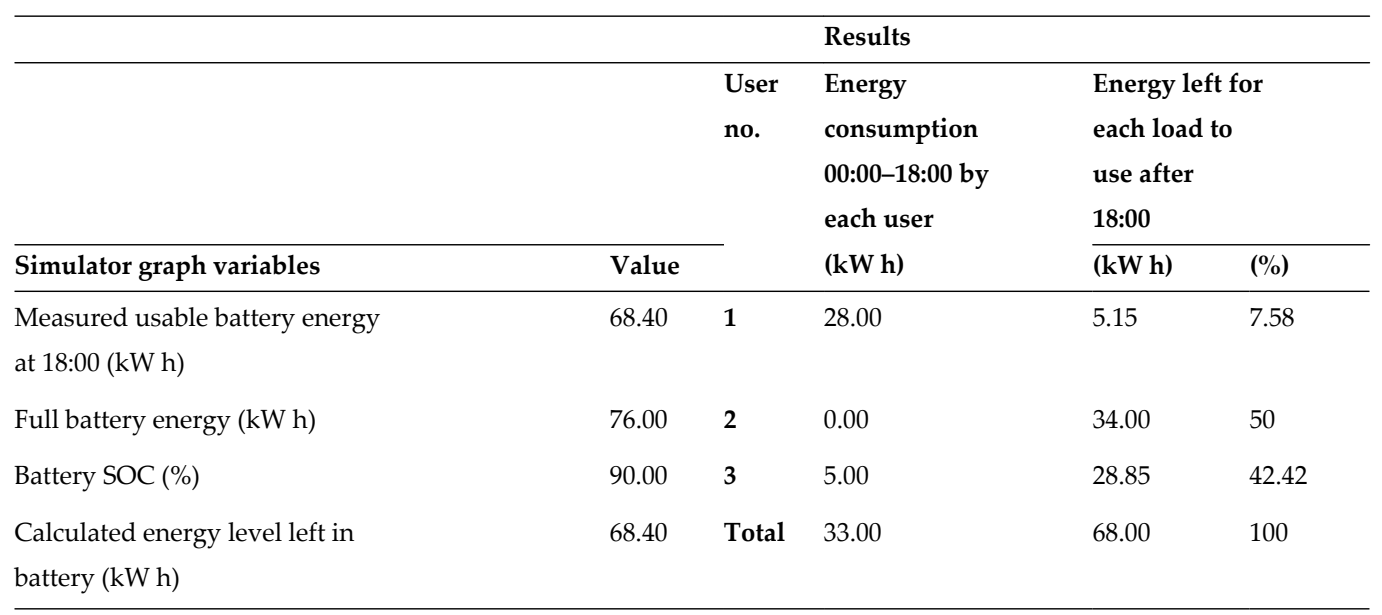

Table 5. Scenario A: result summary.

\section{Conclusions}

An energy reserve share concept was introduced by the development of a control algorithm on the Matlab software platform. The model has been developed in such a way that the Matlab simulation user can interact with the model via the model parameters. The slower real-time speed of model simulation also allows the user to see how parameters change while control processing is performed.

Two scenarios were set up to test if the developed algorithm is functional and to verify that mathematically the charge share formula executes grid control correctly. The purpose of the algorithm is to facilitate the allocation of electric energy among the grid users at 18:00 $\mathrm{h}$, to allow the use of energy up to 24:00 h. Battery SOC and user energy consumption during the day are the determining factors for apportioning the available stored energy among the users.

Scenario A showed a result where equal energy from the remaining SOC was shared between the grid users. Scenario B was setup with variable load changes over a 24-h period. In Scenario $\mathrm{B}$, the allocation of stored energy was done in accordance with the users' energy consumption 
between 00:00 $\mathrm{h}$ and 18:00 $\mathrm{h}$. Users with a high amount of energy consumption before 18:00 $\mathrm{h}$ were allocated a smaller portion of energy from the available stored energy, while users of a low amount of energy (small power users) were allocated a larger portion of energy from the remaining available energy stored.

Islanded MAS grids are affected by a limited energy storage capacity. By using this concept for allocating stored energy for rural-electric applications, electric energy can be divided among multiple users in accordance with their daily use and the remaining amount of available energy stored in the battery.

\section{Author details}

Arno Vosloo and Atanda K. Raji*

*Address all correspondence to: rajia@cput.ac.za

Cape Peninsula University of Technology, Cape Town, South Africa

\section{References}

[1] Schnitzer, D., Lounsbury, D., Carvallo, J., Deshmukh, R., Apt, J., Kammen, D. (2014). Microgrids for rural electrification. IEEE Smart Grid. [Online] Available from http:// smartgrid.ieee.org/april-2014/1071-microgrids-for-rural-electrification [Accessed: 15 May 2014].

[2] Moussavou, A.A.A., Adonis, M., Raji, A. (2015). Microgrid energy management system and its control strategy. International Conference on Industrial and Commercial Use of Energy (ICUE 2015), Belmont Square Conference Centre, Rondebosch, 17th-19th August, 2015, Cape Town, South Africa, pp. 147-154.

[3] Business Times. (2014). It doesn't pay to connect Africa's poor to the grid. [Online] Available: http://www.bdlive.co.za/businesstimes/2014/05/04/the-chatter-it-doesn-tpay-to-connect-africa-s-poor-to-the-grid.

[4] Mcgroarthy, P. (2012). Power to more people. The Journal Report: Innovations in Energy. Available: http://online.wsj.com/articles/.

[5] Mingambo, B.G., Raji, A.K., Kahn, M.T.E. (2014). Design of a dc microgrid system for rural electrification in South Africa. Journal of Energy of Southern Africa, 25(4), pp. 9-14.

[6] Raji, A.K., Kahn, M.T.E. (2011). Distributed energy resources for domestic electricity users. Domestic Use of Energy Conference, Cape Town, South Africa, 11-12 April, 2011, pp. 138-144. 
[7] Phys.Org, Oct 04 (2013). Bringing sustainable electricity to rural African communities. [Online] http://phys.org/news/2013-10-sustainable-electricity-rural-african.html [Accessed: 15 May 2014].

[8] Manickavasagam, K., Nithya, M., Priya, K., Shruthi, J., Krishnan, S., Misra, S., Manikandan, S. (2011). Control of distributed generator and smart grid multi-agent system. In: Electrical Energy Systems (ICEES), 1st International Conference, pp. 212-217.

[9] Logenthiran, T., Srinivasan, D., Khambadkone, A. M., Aung, H. N. (2010). Multi-agent system (MAS) for short-term generation scheduling of a microgrid. IEEE ICSET Sri Lanka. [Online], pp. 1-6. Available from: http://ieeexplore.ieee.org.ezproxy.cput.ac.za/ stamp/stamp.jsp?tp=\&arnumber=5684943 [Accessed: 03 May 2014].

[10] Potty, K.A., Keny, P., Nagarajan, C. (2013). An intelligent microgrid with distributed generation. IEEE Innovative Smart Grid Technologies - ISGT Asia. [Online]. pp. 1-5. Available from: http://ieeexplore.ieee.org.ezproxy.cput.ac.za/stamp/stamp.jsp? tp=\&arnumber=6698755. [Accessed: 03 May 2014].

[11] Jian, Z., Qian, A., Chuanwen, J., Xingang, W., Zhanghua, Z., Chenghong, G. (2009). The application of Multi Agent System in Microgrid coordination control. Sustainable Power Generation and Supply, Supergen. pp. 1-6.

[12] Li, W., Mou, X., Zhou, Y., Marnay, C. (2012). On voltage standards for DC home microgrids energized by distributed sources. In: 7th International Power Electronics and Motion Control Conference - ECCE Asia. June 2-5, 2012, Harbin, China. IEEE. pp. 22822286.

[13] Chaouachi, A., Kamel, R.M., Andoulsi, R., Nagasaka, K. (2013). Multiobjective intelligent energy management for a microgrid. Industrial Electronics, IEEE Transactions [Online], 60(4). pp. 1688-1699. Available from: http://ieeexplore.ieee.org.ezproxy.cput.ac.za/stamp/stamp.jsp?tp=\&arnumber=6157610 [Accessed: 06 May 2014].

[14] Chaouachi, A., Kamel, R.M., Andoulsi, R., Nagasaka, K. (2013). Multiobjective intelligent energy management for a microgrid. Industrial Electronics, IEEE Transactions [Online], 60(4), pp. 1688-1699.

[15] Stluka, P., Godbole, D., Samad, T. (2011). Energy management for buildings and microgrids. In: 50th IEEE Conference on Decision and Control and European Control Conference (CDC-ECC), Orlando, FL, USA, December 12-15, 2011.

[16] Michaelson, D., Mahmood, H., Jiang, J. (2013). A predictive energy management strategy with pre-emptive load shedding for an islanded PV-battery microgrid. In: Industrial Electronics Society, IECON 2013 - 39th Annual Conference of the IEEE, Vienna. 10-13 November 2013. pp. 1501-1506. 
[17] Polycarpou, L. (2013). The microgrid solution [Online] 15 May 2013. Available from http://blogs.ei.columbia.edu/2013/05/15/the-microgrid-solution/ [Accessed: 4 June 2014].

[18] Ramesh, R., Karan, K., Vineeth, V., Dhiwaakar, P., (2013). Implementation of Arduinobased multi-agent system for rural Indian microgrids. In: IEEE PES Innovative Smart Grid Technologies (ISGT). Chennai, India.

[19] Palma-Behnke, R., Reyes, L., Jiménez-Estévez, G. (2012). Smart grid solutions for rural areas. Power and Energy Society General Meeting, 2012 IEEE. 22-26 July 2012. pp. 1-6.

[20] Meiqin, M., Wei, D., Chang, L. (2011). Multi-agent based simulation for Microgrid energy management. In: 8th International conference on Power Electronics and ECCE. Asia. May 30 2011-June 3 2011. IEEE Conference Publications. pp. 1219-1223.

[21] Boynuegri, A. R., Yagcitekin, B., Baysal, M., Karakas, A., Uzunoglu, M. (2013). Energy management algorithm for smart home with renewable energy sources. In: Power Engineering, Energy and Electrical Drives (POWERENG), 2013 Fourth International Conference. 13-17 May 2013. pp. 1753-1758.

[22] Yen-Haw, C., Yen-Hong, C., Ming-Che, H. (2011). Optimal energy management of microgrid systems in Taiwan. In: IEEE PES Innovative Smart Grid Technologies (ISGT). [Online]. pp. 1-9.

[23] Kennedy, J., Ciufo, A., Agalgaonkar, A. (2012) Intelligent load management in microgrids. In: Power and Energy Society General Meeting, IEEE San Diego. July 22-26 2012. pp. $1-8$.

[24] Hajimohamadi, N., Bevrani, H. (2013). Load shedding in microgrids. In: Electrical Engineering (ICEE), 2013 21st Iranian Conference, Mashhad. 14-16 May 2013. pp. 1-6.

[25] Vosloo, A., Raji, A. Intelligent central energy management system for remote community microgrid. In: International Conference on Domestic Use of Energy (DUE), Cape Town, South Africa, 31st March-1st April, 2015, pp. 137-140.

[26] Qin, Q., Chen, Z., Wang, Z. (2012). Overview of microgrid energy management system research status. In: Power Engineering and Automation Conference (PEAM), IEEE. Wuhan. 18-20 September. pp. 1-4.

[27] Xinhua, L., Xutang, Z., Wenjian, L. (2007). Integration of CAPP and CAFD based agent technology. In: International Conference on Mechatronics, Kumamoto, Japan, 8-10 May, 2007.

[28] Hernandez, F., Canesin, C.A., Zamora, R., Martina, F., Srivastava, A.K. (2013). Energy management and control for islanded microgrid using multi-agents. In: North American Power Symposium (NAPS), Manhattan, KS, 22-24 September, pp. 1-6. 
[29] Andreadis, G., Bouzakis, K.D., Klazoglou, P., Niwtaki, K. (2014). Review of agent-based systems in the manufacturing section. Universal Journal of Mechanical Engineering, 2(2). pp. 55-59.

[30] GeoSun Africa. (2014). Updated satellite maps of South Africa's solar resource now available. [Online] Available from - http://geosun.co.za/ [Accessed: 17 January 2015].

[31] Jimeno, J., Anduaga, J., Oyarzabal, J., Gil de Muro, A., (2010). Architecture of a microgrid energy managements system. In: European Transaction on Electrical Power, Spain. 26 April. pp. 1142-1158.

[32] Solar Direct (2015). Solar electric system sizing. [Online] Available from http://solardirect.com/pv/systems/gts/gts-sizing-array.html [Accessed: 20 January 2015].

[33] Back to the source. (2015). Cape Solar Power. [Online] Available from: http:// www.backtothesource.co.za/index.php/cape-solar-power/ [Accessed: 20 January 2015].

[34] Chang, J. Jai, S (2009) Modeling and application of wind-solar energy hybrid power generation system based on Multi-Agent Technology. In Proceedings of the eighth International Conference on Machine Learning and Cybernetics. Baoding, 12-15 July 2009. pp. 1754-1758. 
Chapter 5

\title{
Allocation of Distributed Generation for Maximum Reduction of Energy Losses in Distribution Systems
}

\author{
Juan A. Martinez-Velasco and Gerardo Guerra \\ Additional information is available at the end of the chapter \\ http://dx.doi.org/10.5772/62842
}

\begin{abstract}
The analysis of actual distribution systems with penetration of distributed generation requires powerful tools with capabilities that until very recently were not available in distribution software tools; for instance, probabilistic and time mode simulations. This chapter presents the application of parallel computing to the allocation of distributed generation for maximum reduction of energy losses in distribution system when the system is evaluated during a given period (e.g., the target is to minimize energy losses for periodsequalorlonger than1year).Thesimulationshavebeencarried outusingOpenDSS, a freely available software tool for distribution system studies, when it is driven as a COM DLL from MATLAB using a multicore installation. The chapter details a MATLABOpenDSS procedure for allocation of photovoltaic $(\mathrm{PV})$ generation in distribution systems using a parallel Monte Carlo approach and assuming that loads are voltage-dependent. The main goals are to check the viability of a Monte Carlo method in some studies for which parallel computing can beadvantageously applied and propose a simple procedure for minimization of energy losses in distribution systems.
\end{abstract}

Keywords: distribution system, distributed generation, long-term evaluation, loss minimization, Monte Carlo method, parallel computing

\section{Introduction}

Distributed generation (DG) can provide backup power, support voltage, reduce losses, improve local power quality and reliability, provide ancillary services, and defer distribution system upgrade [1-3]. Modeling of renewable generation raises several challenges, since capabilities for representing intermittent generators, voltage-control equipment, or multiphase unbalanced systems are required. In addition, studies of systems with intermittent nondispatchable resources must be based on a probabilistic approach and calculations 
performed over time periods that may range from minutes to years. Voltage-dependent load models with random variation can also be needed. These issues complicate the study of power distribution systems since software tools have to combine new analysis capabilities with a high number of models for representing various generation technologies, besides the conventional distribution system components, and include capabilities for time mode calculations [4].

One potential application of a power flow simulator is the optimum allocation of distributed generation. This study can be seen from two different perspectives:

- From the independent producer's point of view, the goal is to optimize the benefit. Although the utility will usually impose some constraints to the generation units to be connected to its system (e.g., a maximum rated power), it can be assumed by default that the units can be connected to any node of the system. Therefore, the optimization approach will be in general a feasibility study whose main goal is to check the viability of the installation, select the most economical size (irrespective of the location), and in case of dispatchable units, the control strategy that will maximize the benefit; see for instance $[5,6]$.

- From the utility's point of view, the goal is to maximize the positive impact of distributed generation (e.g., voltage support, energy losses, investment deferring) and minimize or avoid those aspects that can negatively affect the system performance (e.g., miscoordination of protective devices, overvoltages during low load periods); see for instance [7].

This chapter presents a procedure for estimating the size and location of photovoltaic (PV) generators whose connection to the distribution system will minimize system losses.

The following aspects have to be accounted for:

- Given the intermittent and random nature of PV generation, the variable to be minimized is the energy loss, not the power loss. Since power demand and generation are not coincident, the optimum allocation of distributed generators with the goal of minimizing power losses is meaningless.

- The optimum allocation of distributed generators must be carried out taking into account some constraints, namely, thermal limits of distribution system components and the maximum voltage that can be accepted in the system under study.

- In a deregulated market in which independent producers connect their generation units to the distribution system without considering their impact on energy losses, the connection of generation units will usually be random and not be made with the target assumed in this chapter. In other words, assuming that either utilities or independent producers will select sizes and locations of generators with the maximum reduction of energy losses in mind is not realistic. Therefore, the goal of this study is the theoretical estimation of the maximum energy loss reduction that can be achieved with the connection of PV generators.

- Since the goal is to maximize energy loss reduction, two other aspects, in addition to sizes and locations of generators, have to be considered: the period during which energy losses must be minimized and the time at which every PV generator must be connected to the distribution system. 
Several strategies have been proposed to optimally allocate DG; for instance, loss minimization [8], system update minimization [9], risk minimization [10], or DG capability minimization [11]. A significant activity has been dedicated to this purpose during the last decade; for a summary of the works related to optimum allocation of DG, see references [12] and [13].

Although some works have been made in this field using probabilistic methods [14, 15], not much has been done with a full model of the distribution system (e.g., a multiphase model including load and generation). A Monte Carlo method is a natural approach when uncertainties are involved and some variables are random/intermittent. However, the application of a pure Monte Carlo method can be time consuming if many DG units have to be allocated in large distribution systems. Multicore computers and software that take advantage of their capabilities can be used to significantly reduce the computation time.

This chapter presents a procedure based on a parallel Monte Carlo method for estimating the size and location of PV generators that can minimize distribution energy losses during a period of time, assuming voltage-dependent loads.

Two evaluations are considered in this chapter:

1. Short-term evaluation: The size and location of generators are estimated in order to minimize distribution energy losses during 1 year. If more than one generator is to be connected, then all generators are simultaneously connected at the beginning of the year.

2. Long-term evaluation: The size and location of generators are estimated in order to minimize distribution system losses during a period longer than 1 year. In this chapter, the evaluation period will be 10 years; up to six generators will be connected, and their connection will be sequential (i.e., one generator will be connected at the beginning of consecutive years during the first 6 years).

Section 2 presents the test system studied in this chapter. The procedure developed for shortterm evaluation (i.e., minimization of energy losses during 1 year) is detailed in Section 3 . The section includes a description of the Monte Carlo approach, its implementation for a multicore environment, and the results obtained when up to four PV generators are allocated. The section also proposes a refinement of the Monte Carlo method aimed at reducing the number of runs and therefore the computing time. Section 4 details the procedure developed for long-term evaluation (i.e., minimization of energy losses during 10 years) when up to six PV generators are to be sequentially connected. A simplified method for energy loss minimization will be presented in Section 5. Finally, a summary of the main conclusions derived from results presented in this chapter and other works by the authors is presented in Section 6.

\section{Test system}

Figure 1 shows the diagram of the test system. It is a three-phase $60-\mathrm{Hz}$ single-feeder distribution system with a distributed load. The model includes the substation transformer and a simplified representation of the high-voltage system. The system tested is a 500-node radial configuration feeder with the following characteristics: 
- Total feeder length: $30,000 \mathrm{ft}$.

- Number of nodes: 500 (i.e., section length $=60 \mathrm{ft}$ ).

- Node load $=9 \mathrm{~kW}, \mathrm{pf}=0.9(\mathrm{lg})$ (i.e., total load $=4500 \mathrm{~kW})$.

The phase conductors of the line are in a flat configuration, and the normal thermal limit is 400 A, with an emergency limit of $600 \mathrm{~A}$.

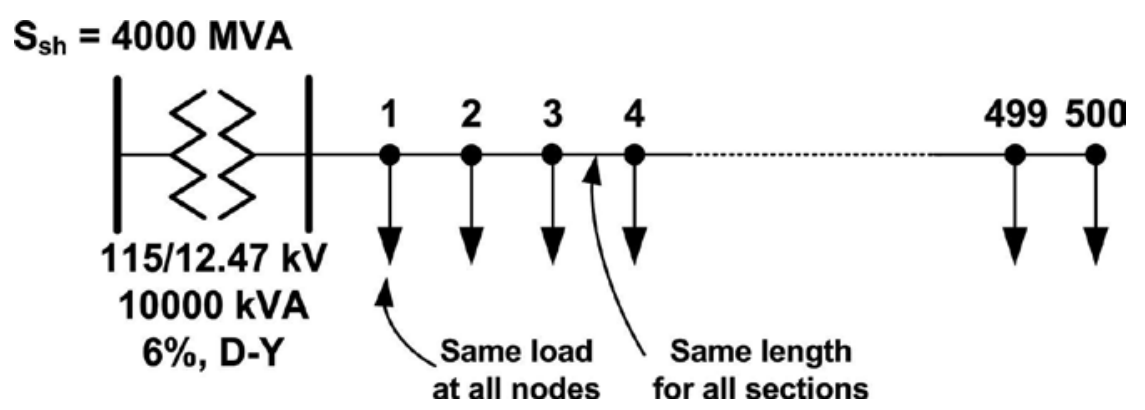

Figure 1. Test system configuration and data.

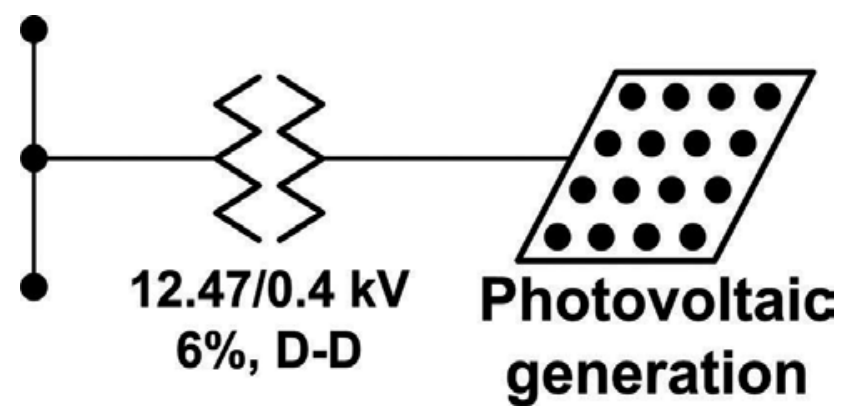

Figure 2. Configuration of a PV generator.

The studies have been carried out with the PV generator model presented in [16]. For the current study, a PV generator is connected to the system through a step-up interconnection transformer (see Figure 2). The rated power of the interconnection transformer is chosen once the rated power of the PV generator/plant has been selected; it is rounded in steps of 50 $\mathrm{kVA}$, and, by default, the short-circuit impedance is $6 \%$. The $X / R$ ratio is 6 .

Table 1 summarizes the information used to obtain PV generation curves. The implemented procedure will be used for obtaining the allocation of PV generators when considering that the shape of each node load is different (although the patterns will exhibit some similarities during the day hours and the week days), the generators only inject active power, and their generation patterns are the same. All node load profiles and PV generation curves have been created using the algorithms presented in [17]. 
Average monthly clearness index

Panel's slope angle

Normal operating cell temperature

Average monthly daily minimum temperatures $\left({ }^{\circ} \mathrm{C}\right)$

Average monthly daily maximum temperatures $\left({ }^{\circ} \mathrm{C}\right)$
$0.41,0.43,0.44,0.47,0.45,0.5,0.51,0.5,0.51,0.5,0.42,0.4$

$35^{\circ}$

$45^{\circ} \mathrm{C}$

$-5.92,-3.9,-0.21,5.09,9.89,14.9,17.1,16.6,12.6,6.95,1.97,-3.76$

$2.58,5.5,11.1,18.9,25.1,29.9,31.6,30.7,26.7,19.8,11.2,4.13$

Table 1. Summary of solar resources.

Figure 3 provides an example of curve shapes for representing load and PV generation derived from the procedures developed by the authors for a given period of the year. The two curves are depicted with a different scale for active power values.

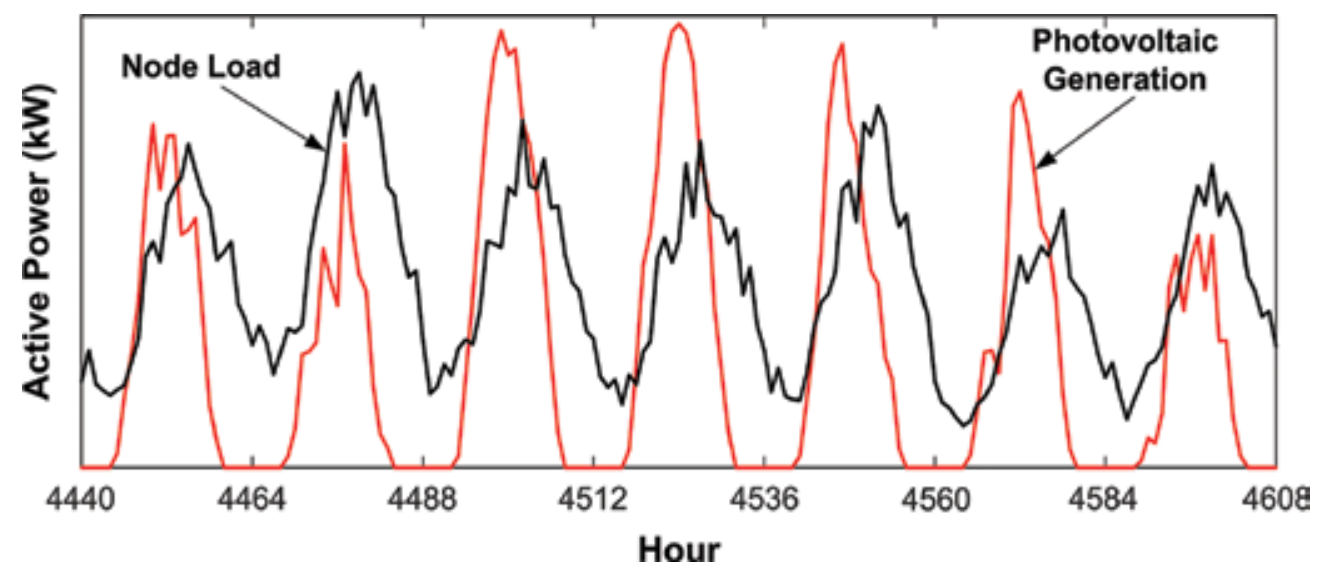

Figure 3. Curve shapes for node loads and PV generation.

The radial feeder configuration has been chosen because the solution to this problem is well known when the load is constant, voltage independent, and uniformly distributed. The optimum allocation of capacitor banks in a distribution feeder with uniformly distributed load has been thoroughly analyzed [18]. The analysis is similar when the goal is to minimize losses by installing generation units that only inject active power [8]. This system configuration can be useful for validating test cases whose results are obtained by means of a Monte Carlo method [19].

\section{Maximum reduction of energy losses--short-term evaluation}

\subsection{Introduction}

This section presents the application of a Monte Carlo method to the estimation of the size and location of the PV generators that will minimize energy losses in distribution systems during 
1 year without including substation losses. The section details the method, its implementation in a MATLAB-OpenDSS environment, and its application to the test system presented above using single-core and multicore computations. Later, a refinement of the Monte Carlo for multicore computation aimed at decreasing the computing time is proposed.

\subsection{Application of the Monte Carlo method}

A 1-year evaluation period has been considered for short-term evaluation. The study is carried out assuming that generation units will be simultaneously connected at the beginning of the evaluation period, taking into account some operational constraints (e.g., there are a thermal limit for each system line section and a maximum voltage that should not be exceeded).

Input data for the present study includes system parameters and time variation of loads and generations. Random variables to be generated during the application of the Monte Carlo method are locations and sizes of the generation units. This latter aspect can be rigorously made by considering that the generation pattern depends on the area/node where the generator is located. In this chapter, generation units are PV arrays that only inject active power into the system. It is also assumed that the solar radiation is the same for each generator.

The procedure has been implemented taking into account certain rules when choosing locations and sizes for the generators. The rules as well as the general procedure used in this work for short-term (1 year) evaluations are detailed below [20]:

1. PV generators can be connected to any system node. The locations to which generators will be connected are determined by generating as many uniformly distributed random values as units to be allocated.

2. Once the locations are known, the rated power values are determined. Beforehand, the user has to fix the maximum generation power that can be connected to system nodes taking into account some constraints (e.g., the maximum thermal limit of feeder sections). Remember that all line sections are equal in the test system. This step is carried out as follows:

- When only one generator is to be allocated, the maximum rated power is determined according to the following steps: (i) calculate the maximum noncoincident active power (i.e., the active power value that results from adding the active rated power of all load nodes); (ii) check the maximum power that can be carried by the feeder sections; (iii) compare the previous power values and choose the minimum one; this will be the maximum rated power a generation unit can be assigned during the Monte Carlo execution. Then generate a random number uniformly distributed between 0 and 1 , and multiple it by the above value.

- When two or more units are to be installed, the following changes are introduced in the procedure:

- Generate an independent uniformly distributed random value for the initial rated power of each generation unit using the maximum rated power fixed for every node as the upper end point of each uniform distribution. 
- Compare the maximum rated powers for all the chosen locations and choose the maximum value, $P_{D G \_M A X}$.

- Generate the penetration factor as a uniformly distributed random number between 0 and 1 . Multiply the maximum value found in the previous step by this penetration factor; the result will be the overall rated power of distributed generators.

$$
\sum_{i=1}^{N G} P_{D G_{-} i}=p f \cdot P_{D G_{-} M A X}
$$

where $N G$ is the number of generation units under evaluation, $P_{D G_{-}} i$ is the rated power of unit $i, p f$ is the penetration factor, and $P_{D G_{-} M A X}$ is the maximum DG-rated power found in the previous step.

- Calculate the scale factor, $s f$, from the initial rated powers as follows:

$$
s f=\frac{p f \cdot P_{D G_{-} M A X}}{\sum_{i=1}^{N G} P_{D G i n i t_{-} i}}
$$

where $P_{\text {DGinit } i}$ is the initial rated power for unit $i$.

- Obtain generator-rated powers by scaling initial rated powers:

$$
P_{D G_{-} i}=s f \cdot P_{D G I_{-} i}
$$

Note that the order in which random values for locations and rated powers are generated matters: first, the location nodes; afterwards, the rated powers.

3. Perform the load flow calculation. Neglect a case if one of the following conditions is satisfied: (i) the voltage at one node exceeds the fixed maximum value; (ii) the current through one or more system sections is above the thermal limit.

4. Stop the procedure when the specified number of runs or samples (according to the terminology of the Monte Carlo method) is reached.

The combination of rated powers and locations that produces the minimum energy losses and meets the technical constraints will be selected as the solution. The number of executed runs must be large enough to ensure that the estimated solution is near enough to the global minimum. In this work, it is assumed the method has converged when the variations of the estimated minimum of system energy losses are within a margin of $1 \%$.

\subsection{Implementation of the procedure}

The procedure has been implemented in OpenDSS, a simulation tool for electric utility distribution systems, which can be used as both a stand-alone executable program and a COM 
DLL that can be driven from some software platforms [21]. In this study, the program is driven from MATLAB, which is used to calculate the random variables and control the execution of the procedure. The implementation of the procedure, when using parallel computing, is schematized in Figure 4, and is valid for any number of cores. MATLAB capabilities are used to distribute Monte Carlo runs between cores.

\section{Data Input}

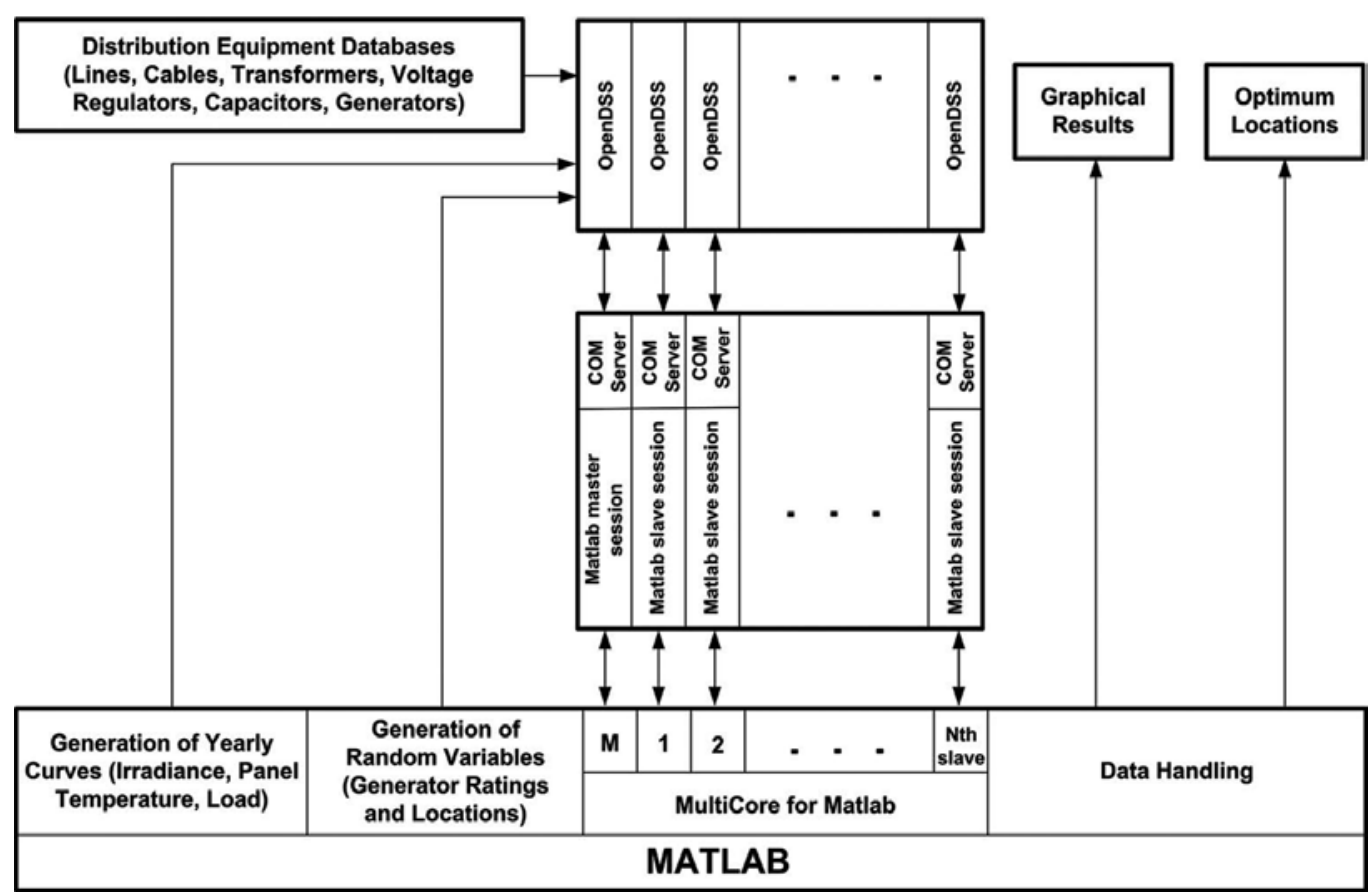

Figure 4. Block diagram of the implemented procedure.

The implementation of the procedure for any number of cores is based on the library of MATLAB modules developed by Buehren [22]. Load and intermittent solar generation curves were generated by means of the algorithms detailed in [17].

\subsection{Simulation results using single-core computing}

Table 2 summarizes the operating conditions in the system before any generation unit is connected. Three different models are used to represent loads (constant power model, constant impedance model, and ZIP model). The ZIP load model is defined as a combination of constant power, constant current, and constant impedance load models [23]. When this model is used, weighting factors are assigned to specify active and reactive powers for each of these three components, being the sum of the weighting factors equal to unity for both active and reactive powers. For the present study, each load component has been assigned a weighting factor equal to $1 / 3$ for both active and reactive powers; this means that $1 / 3$ of the load behaves 
respectively as a constant power, constant current, and constant impedance load. For more details about component fractions and power factors of common actual loads, see [23]. The minimum voltage value in this system during the year of study is $0.9561 \mathrm{p} . \mathrm{u}$. and occurs with the constant power load model.

\begin{tabular}{llll}
\hline & Constant power load model & Constant impedance load model & ZIP load model \\
\hline Energy from substation $(\mathrm{kWh})$ & $16,914,396.5$ & $16,503,855.3$ & $16,702,520.9$ \\
Energy losses $(\mathrm{kWh})$ & $161,732.4$ & $151,198.0$ & $156,245.2$ \\
Energy losses $(\%)$ & 0.9561 & 0.9161 & 0.9354 \\
\hline
\end{tabular}

Table 2. Short-term evaluation (1 year)—operating conditions without PV generation.

Table 3 summarizes the results obtained after applying the procedure with different number of runs when allocating one, two, and four PV generators. The results presented in Table 3 were obtained by assuming a constant power load model. The maximum accepted voltage in the feeder for this and any other study presented here is $1.05 \mathrm{p} . \mathrm{u}$. These results clearly prove that the number of runs has to be increased with the number of PV generators to be allocated. The results presented in Table 3 show clear tendencies for one and two generation units; they indicate that the number of executed runs is enough for these two cases. However, the values obtained for four units present important variations from one execution to another; this is an indication that more runs are necessary to identify the correct values or tendencies for locations and rated powers, although energy losses are within the desired margin (i.e., 1\%). Figure 5 depicts the results corresponding to the allocation of one generation unit after 5040 runs. This figure illustrates the way in which minimum energy losses can be estimated.

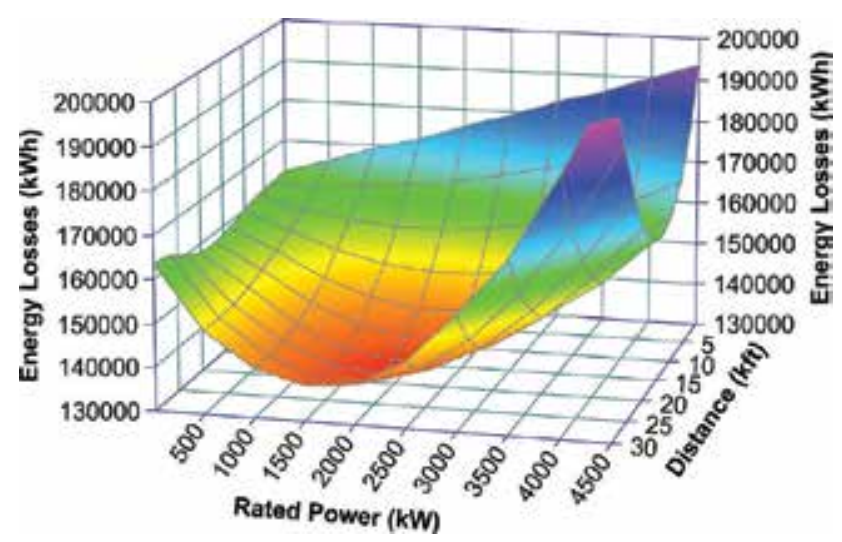

Figure 5. Optimum allocation of a single PV generator-5040 runs.

The complete results with the three load models are shown in Table 4. A behavior similar to that obtained with the previous study can be identified: the results for one and two generation units show clear tendencies, but when allocating four units, the results are not conclusive and 
might require a higher number of runs. These results prove again that the number of runs has to be increased with the number of generation units to be allocated. The differences between energy loss values obtained with different numbers of runs are very small in all studies (always within the $1 \%$ margin); therefore, it can be expected that the global minimum will not present a significant variation with respect to the values found here. It has been discussed that more runs are required to establish a well-defined tendency for more than two generation units; however, one must consider if the increment in computational time is justified when assessing the improvement in energy losses obtained with a larger number of runs.

\begin{tabular}{|c|c|c|c|c|}
\hline \multicolumn{5}{|c|}{ One PV generator } \\
\hline Runs & & 2520 & 5040 & 10,080 \\
\hline \multirow[t]{2}{*}{ Unit 1} & Node & 381 & 376 & 383 \\
\hline & Power $(\mathrm{kW})$ & 1954.7 & 2005.2 & 1956.4 \\
\hline \multicolumn{2}{|c|}{ Energy losses (kWh) } & 134948.4 & 134995.5 & 134964.8 \\
\hline \multicolumn{2}{|c|}{ Energy losses (\%) } & 0.7991 & 0.7994 & 0.7992 \\
\hline \multicolumn{5}{|c|}{ Two PV generators } \\
\hline Runs & & 20,040 & 30,000 & 40,080 \\
\hline \multirow[t]{2}{*}{ Unit 1} & Node & 279 & 284 & 290 \\
\hline & Power $(\mathrm{kW})$ & 1097.4 & 1192.8 & 970.7 \\
\hline \multirow[t]{2}{*}{ Unit 2} & Node & 437 & 426 & 426 \\
\hline & Power $(\mathrm{kW})$ & 1093.3 & 1003.1 & 1186.7 \\
\hline \multicolumn{2}{|c|}{ Energy losses (kWh) } & 133532.0 & 133607.0 & 133571.8 \\
\hline \multicolumn{2}{|c|}{ Energy losses (\%) } & 0.7908 & 0.7912 & 0.7910 \\
\hline \multicolumn{5}{|c|}{ Four PV generators } \\
\hline \multicolumn{2}{|l|}{ Runs } & 40,080 & 60,000 & 80,040 \\
\hline \multirow[t]{2}{*}{ Unit 1} & Node & 328 & 229 & 220 \\
\hline & Power $(\mathrm{kW})$ & 869.0 & 518.1 & 455.4 \\
\hline \multirow[t]{2}{*}{ Unit 2} & Node & 329 & 353 & 307 \\
\hline & Power $(\mathrm{kW})$ & 186.1 & 548.7 & 796.8 \\
\hline \multirow[t]{2}{*}{ Unit 3} & Node & 336 & 387 & 428 \\
\hline & Power $(\mathrm{kW})$ & 230.0 & 701.2 & 683.7 \\
\hline \multirow[t]{2}{*}{ Unit 4} & Node (kW) & 449 & 490 & 448 \\
\hline & Power $(\mathrm{kW})$ & 823.3 & 414.0 & 411.7 \\
\hline \multicolumn{2}{|c|}{ Energy losses (kWh) } & 132991.7 & 132927.7 & 132873.3 \\
\hline \multicolumn{2}{|c|}{ Energy losses (\%) } & 0.7876 & 0.7872 & 0.7869 \\
\hline
\end{tabular}

Table 3. Short-term evaluation (1 year)—allocation of PV generators (constant power load model). 


\begin{tabular}{|c|c|c|c|c|}
\hline \multicolumn{2}{|l|}{ Scenario } & \multirow{2}{*}{$\begin{array}{l}\text { Constant power } \\
\text { load model }\end{array}$} & \multirow{2}{*}{$\begin{array}{l}\text { Constant impedance } \\
\text { load model } \\
376\end{array}$} & \multirow{2}{*}{$\begin{array}{l}\text { ZIP load model } \\
385\end{array}$} \\
\hline One PV generator (5040 runs) & Node & & & \\
\hline & Rated power $(\mathrm{kW})$ & 2005.2 & 1868.8 & 1913.3 \\
\hline & Energy losses (kWh) & 134995.5 & 127772.1 & 131247.9 \\
\hline \multirow[t]{3}{*}{ Two PV generators (40080 runs) } & Nodes & $290-426$ & $309-420$ & $269-425$ \\
\hline & Rated powers $(\mathrm{kW})$ & $970.7-1186.7$ & $1230.7-828.5$ & $915.0-1189.7$ \\
\hline & Energy losses (kWh) & 133571.8 & 126547.9 & 129919.6 \\
\hline \multirow[t]{5}{*}{ Four PV generators (80040 runs) } & Nodes & $220-307$ & $277-338$ & $308-379$ \\
\hline & & $428-448$ & $435-471$ & $432-448$ \\
\hline & Rated powers $(\mathrm{kW})$ & $455.4-796.8$ & $422.6-957.3$ & $645.6-548.8$ \\
\hline & & $683.7-411.7$ & $339.2-418.5$ & $376.0-369.9$ \\
\hline & Energy losses (kWh) & 132873.3 & 125827.0 & 129231.6 \\
\hline
\end{tabular}

Table 4. Short-term evaluation (1 year)—comparison of simulation results.

Some interesting conclusions are derived from the comparison of these results with those obtained in Ref. [19]. Although the test system and load are the same in both studies, there are some differences in sizes and locations of the PV generators to be allocated; for instance, the difference between the sizes of the generators obtained in both studies when only one unit has to be allocated is about $25 \%$.

There are several reasons for these differences:

- The target in [19] included the energy losses in the substation transformer, while the target in this chapter is to minimize energy losses in the feeder, without including substation losses. This is obviously an important reason to obtain smaller size values in the current study.

- The model used to represent PV generation is different in both studies. Reference [19] was based on a basic generator model represented only by the active and reactive powers it injects into the grid, while the current study is based on a more detailed custom-made PV generator model that is an expanded version on that available in OpenDSS [16]. Take into account that the new PV generator model includes the interconnection transformer, in which some losses are always caused.

- The results obtained in [19] were derived by means of curve shapes obtained from HOMER [5], while the results presented here are based on algorithms developed for OpenDSS application [17]. Although PV generation curves obtained with HOMER and with the MATLAB application developed by the authors are very similar, the solar resources (i.e., information like that in Table 2) are different in both studies. In addition, discrepancies are significant in the load curves. The load curve shapes obtained with the algorithm presented in [17] were derived from actual measurements that could be considered as representative of some types of loads (e.g., residential, commercial). 
Somehow, this discussion supports important conclusions: the results are very much dependent on the component models, and the allocation of generation units must be based on the minimization of energy losses, not on power losses. The results can be different even when load ratings are equal and if the load shapes are different (e.g., same ratings but different time variation).

\subsection{Simulation results using multi-core computing}

A very important aspect to consider is the reduction of time that can be achieved when using a multicore installation. Table 5 provides the computing times that are required for simulating the test system when only one unit has to be allocated, as well as the corresponding energy losses, using single-core and multicore computations. As expected, the achieved reduction of simulation time is significant and almost proportional to the number of cores.

\begin{tabular}{llll}
\hline Scenario & Load model & Single core & $\mathbf{6 0}$ cores \\
\hline One PV generator (5040 runs) & Constant power load model & $134978.0(\mathrm{kWh})$ & $134995.5(\mathrm{kWh})$ \\
& & $36.46(\mathrm{~h})$ & $0.92(\mathrm{~h})$ \\
& Constant impedance load model & $127806.0(\mathrm{kWh})$ & $127772.1(\mathrm{kWh})$ \\
& & $35.46(\mathrm{~h})$ & $0.86(\mathrm{~h})$ \\
& ZIP load model & $131270.8(\mathrm{kWh})$ & $131247.9(\mathrm{kWh})$ \\
& & $43.66(\mathrm{~h})$ & $1.00(\mathrm{~h})$ \\
\hline
\end{tabular}

Table 5. Simulation results using single-core and multicore computing.

\subsection{Refinement of the Monte Carlo method}

Figure 6 illustrates the way in which the Monte Carlo method can be applied to this particular study when only one generation unit has to be connected [19]. Every cross within the square is a combination of the two random numbers that are generated for each run: the distance with respect to the origin of the feeder at which the generation unit is to be connected, and the corresponding rated power. Remember that the result of concern for the present study is the energy loss obtained with each combination of values.

According to the results presented in Figure 5 and Table 4, not much difference between energy loss values should be expected when the combination of the two random values gives a point that is closely located to a previously simulated point that is not far from the minimum. Therefore, during the generation of random values, these cases do not need to be simulated; so, the number of runs can be significantly reduced. The goal now is to check how much accuracy is lost and how much reduction of time is achieved by neglecting some runs. The approach is similar when more than one generation unit has to be connected. In such a case, all combinations located within a Euclidean distance equal or shorter than $R$ are discarded.

Once some runs have been already computed and simulated, the combination of random values corresponding to run $k$ is not simulated when the following condition is fulfilled: 


$$
\sqrt{x_{i j-i k}^{2}+p_{i j-i k}^{2}} \leq R
$$

where $x_{i j-i k}$ is the distance in percent between the location of the unit $i$ corresponding to $j$ runs (previously simulated) and unit $k$, and $p_{i-i k i}$ is the distance in percent between the rated powers of the unit $i$ corresponding to $j$ runs and unit $k$. $R$ in this work will be $5 \%$.

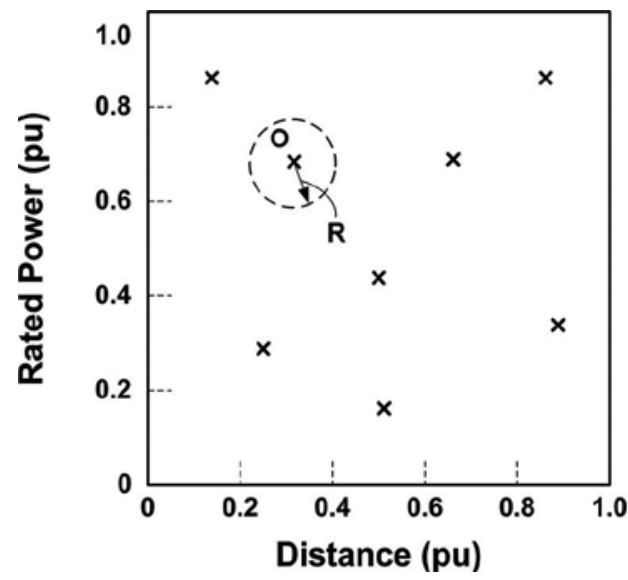

x Combinations already used in calculations

O Combination discarded for calculations $\mathbf{R}=\mathbf{5} \%$ of the feeder length/total power

Figure 6. Generation of random values for energy loss minimization—one generation unit.

When more than one unit is being allocated, the calculation of the minimum Euclidean distance between the combinations of random values corresponding to two different runs is not obvious, since the order in which generation units are numbered does not have to be same when comparing two different runs. In addition, expression (4) has to be checked with respect to any previous combination, which can be time-consuming. A solution to this problem was proposed in [19].

Table 6 shows a summary of results obtained for the test system after applying the new approach. All the results shown in the table were obtained with a 60-core installation. The table compares results obtained by means of the conventional Monte Carlo method and the new approach. According to these results, a significant reduction in computing time can be achieved; as expected, the accuracy decreases as the number of generators increases.

Although some differences between location and power values between the two approaches are very significant, the energy losses obtained when applying the new method are basically the same as that were obtained with the original method. These differences can be justified by looking at the energy losses obtained when a single generation unit is to be allocated (see Figure 5). The surface shown in the figure is very smooth around the minimum, so the difference between energy losses even for nonsmall deviations with respect to the minimum will not be very significant. Note also that the ratio between simulation times with both methods is about 15 when one unit has to be allocated and about 2 when the number of units is four. 


\begin{tabular}{|c|c|c|c|c|}
\hline Number of generators & Scenario & & Conventional & Refined \\
\hline One PV generator (5040 runs) & Constant power & Node & 376 & 375 \\
\hline & load model & Rated powers $(\mathrm{kW})$ & 2005.2 & 1894.5 \\
\hline & & Energy losses (kWh) & 134995.5 & 135221.4 \\
\hline & & Simulation time (h) & 0.92 & 0.06 \\
\hline & Constant impedance & Node & 376 & 376 \\
\hline & load model & Rated powers $(\mathrm{kW})$ & 1868.8 & 1757.4 \\
\hline & & Energy losses (kWh) & 127772.1 & 128051.3 \\
\hline & & Simulation time $(\mathrm{h})$ & 0.86 & 0.06 \\
\hline & ZIP load model & Node & 385 & 385 \\
\hline & & Rated powers $(\mathrm{kW})$ & 1913.3 & 1836.5 \\
\hline & & Energy losses (kWh) & 131247.9 & 131377.2 \\
\hline & & Simulation time $(\mathrm{h})$ & 1.00 & 0.07 \\
\hline Two PV generators (40080 runs) & Constant power & Nodes & $290 / 426$ & $308 / 427$ \\
\hline & load model & Rated powers $(\mathrm{kW})$ & $970.7 / 1186.7$ & 1187.2/959.1 \\
\hline & & Energy losses (kWh) & 133571.8 & 133515.0 \\
\hline & & Simulation time $(\mathrm{h})$ & 7.21 & 1.80 \\
\hline & Constant impedance & Nodes & $309 / 420$ & $285 / 427$ \\
\hline & load model & Rated powers $(\mathrm{kW})$ & $1230.7 / 828.5$ & $1110.2 / 1055.7$ \\
\hline & & Energy losses (kWh) & 126547.9 & 126612.8 \\
\hline & & Simulation time $(\mathrm{h})$ & 7.11 & 1.73 \\
\hline & ZIP load model & Nodes & $269 / 425$ & $302 / 447$ \\
\hline & & Rated powers $(\mathrm{kW})$ & $915.0 / 1189.7$ & $961.6 / 956.5$ \\
\hline & & Energy losses (kWh) & 129919.6 & 129958.8 \\
\hline & & Simulation time $(\mathrm{h})$ & 8.09 & 2.00 \\
\hline Four PV generators (80040 runs) & Constant power & Nodes & $220 / 307$ & $279 / 353$ \\
\hline & load model & & $428 / 448$ & $390 / 466$ \\
\hline & & Rated powers $(\mathrm{kW})$ & $455.4 / 796.8$ & $546.6 / 412.5$ \\
\hline & & & $683.7 / 411.7$ & $642.1 / 487.7$ \\
\hline & & Energy losses (kWh) & 132873.3 & 132813.0 \\
\hline & & Simulation time $(\mathrm{h})$ & 14.50 & 7.07 \\
\hline & Constant impedance & Nodes & $277 / 338$ & $271 / 337$ \\
\hline & load model & & $435 / 471$ & $369 / 474$ \\
\hline & & Rated powers $(\mathrm{kW})$ & $422.6 / 957.3$ & $863.9 / 227.9$ \\
\hline & & & $339.2 / 418.5$ & $658.6 / 456.2$ \\
\hline
\end{tabular}




\begin{tabular}{|c|c|c|c|c|}
\hline \multirow[t]{2}{*}{ Number of generators } & \multicolumn{2}{|l|}{ Scenario } & \multirow{2}{*}{$\begin{array}{l}\begin{array}{l}\text { Conventional } \\
\text { method }\end{array} \\
125827.0\end{array}$} & \multirow{2}{*}{$\begin{array}{c}\begin{array}{l}\text { Refined } \\
\text { method }\end{array} \\
125773.2 \\
\end{array}$} \\
\hline & \multirow{8}{*}{ ZIP load model } & Energy losses (kWh) & & \\
\hline & & Simulation time (h) & 14.03 & 6.84 \\
\hline & & Nodes & $308 / 379$ & $255 / 300$ \\
\hline & & & $432 / 448$ & $354 / 445$ \\
\hline & & Rated powers $(\mathrm{kW})$ & $645.6 / 548.8$ & $426.4 / 819.4$ \\
\hline & & & $376.0 / 369.9$ & $91.7 / 835.5$ \\
\hline & & Energy losses (kWh) & 129231.6 & 129339.1 \\
\hline & & Simulation time (h) & 16.15 & 7.91 \\
\hline
\end{tabular}

Table 6. Simulation results using a refined Monte Carlo method.

\section{Maximum reduction of energy losses--long-term evaluation}

This section presents the results derived from a long-term study aimed at estimating the size and location of PV generators when the target is to minimize the energy losses and the generators are sequentially connected.

Figure 7 shows the variation assumed for the total load during the evaluation period. A different variation for each node load and even negative variations have been assumed. Table 7 shows the year of connection for each PV generator.

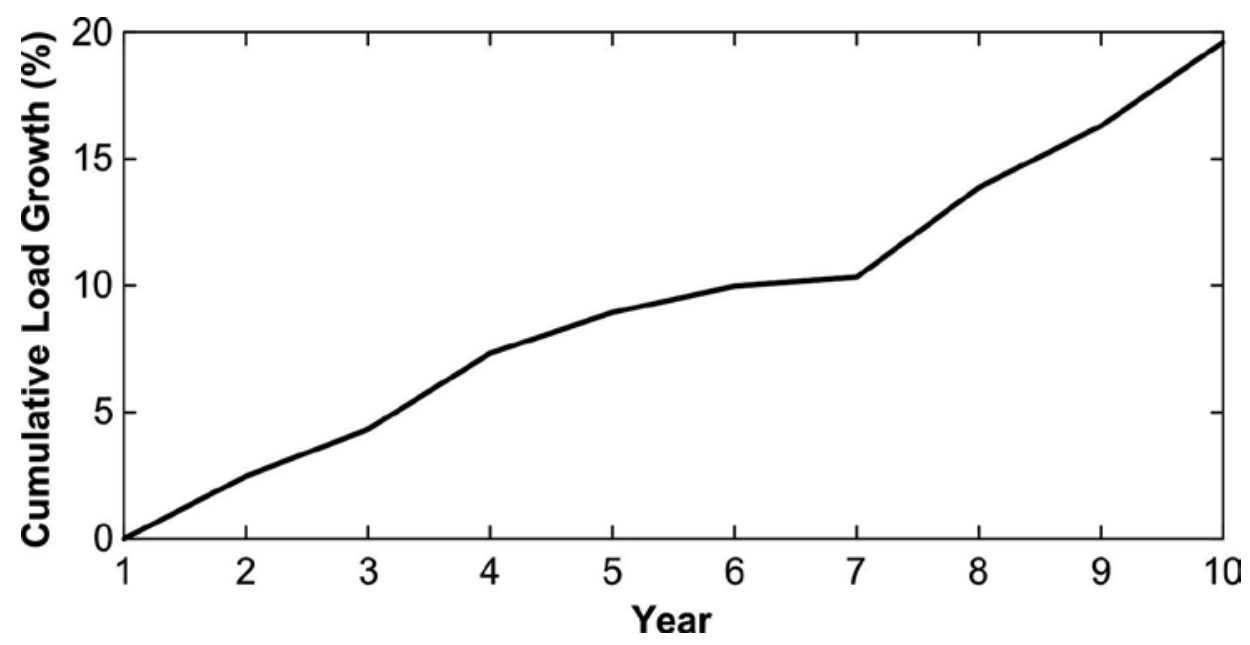

Figure 7. Long-term evaluation-load growth. 


\begin{tabular}{|c|c|c|c|c|c|c|c|c|c|c|}
\hline \multirow[t]{2}{*}{ PV generator } & \multicolumn{10}{|c|}{ Year } \\
\hline & $\overline{1}$ & 2 & 3 & 4 & 5 & 6 & 7 & 8 & 9 & 10 \\
\hline Unit 1 & $\bullet$ & $X$ & $X$ & $X$ & $X$ & - & - & - & - & - \\
\hline Unit 2 & & $\bullet$ & $x$ & $x$ & $x$ & $x$ & - & - & - & - \\
\hline Unit 3 & & & $\bullet$ & $x$ & $x$ & $x$ & $x$ & - & - & - \\
\hline Unit 4 & & & & $\bullet$ & $x$ & $x$ & $x$ & $x$ & - & - \\
\hline Unit 5 & & & & & $\bullet$ & $x$ & $x$ & $x$ & $x$ & - \\
\hline Unit 6 & & & & & & $\bullet$ & $x$ & $x$ & $x$ & $x$ \\
\hline
\end{tabular}

-, Year of connection; X, considered for optimization; -, not considered for optimization.

Table 7. Scenario for long-term evaluation.

The main aspects of the study are summarized below:

- Up to six PV generators will be connected to the test system.

- The optimization period every time a PV unit is added to the system is 5 years. That is, the target upon which the locations and rated powers of the units are selected is the minimization of energy losses during 10 years, taking into account that every unit is connected at the beginning of a year, and its size and location are selected to minimize energy losses during 5 years. According to this, if a unit is connected at the beginning of year 3 , the energy losses to be accounted for are those caused from the beginning of year 3 till the end of year 7 (see Table 7).

The energy losses that correspond to the entire period of evaluation (i.e., 10 years) without PV generation and considering the three types of load models are as follows:

- Constant power load model: 1,950,262.8 kWh.

- Constant impedance load model: 1,803,067.9 kWh.

- ZIP load model: 1,873,210.8 kWh.

The minimum voltage value during this period is 0.9423 p.u., and occurs again when loads are represented by the constant power model.

Table 8 shows the results obtained upon the application of both the conventional Monte Carlo and the refined methods during the period of evaluation (e.g., 10 years). The number of runs to be used every year a PV generator is connected is based on the experience obtained with the previous study. As shown in Table 6, when one generator is to be connected, 5040 runs will usually suffice. Table 9 summarizes the main results corresponding to each load model. The value of $R$ when the refined method is applied is again 5\%. Remember that substation losses are not included. 


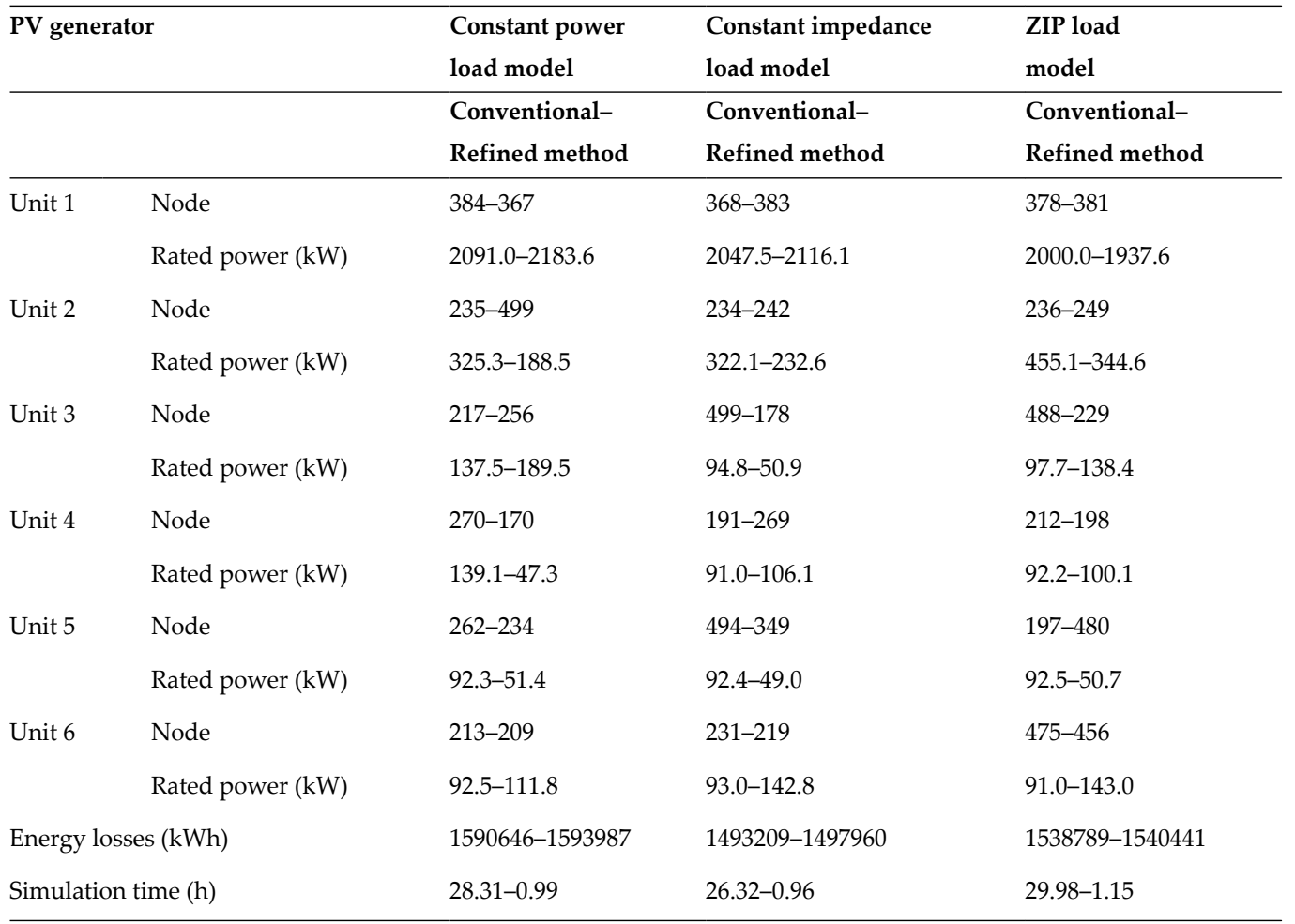

Table 8. Long-term evaluation-comparison of simulation results.

\begin{tabular}{lll}
\hline Load model & $\begin{array}{l}\text { Total generation }(\mathbf{k W}) \\
\text { (Conventional-Refined) }\end{array}$ & $\begin{array}{l}\text { Cumulative energy loss reduction (\%) } \\
\text { (Conventional-Refined) }\end{array}$ \\
\hline Constant power & $2878.0-2772.4$ & $18.439-18.268$ \\
Constant impedance & $2741.0-2697.8$ & $17.185-16.921$ \\
ZIP & $2828.8-2714.6$ & $17.852-17.764$ \\
\hline
\end{tabular}

Table 9. Long-term evaluation—-total generation and energy loss reduction.

The maximum coincident active power measured at the substation terminals for each load model during the period of study is as follows: (1) constant power model $=4021.5 \mathrm{~kW}$, (2) constant impedance model $=3753.5 \mathrm{~kW}$; (3) ZIP model $=3879.7 \mathrm{~kW}$. After connecting six generation units, the total rated power of all PV generators is less than $75 \%$ of the maximum coincident power of the system at the end of the period.

From the results presented in Table 8, one can observe that the maximum rated power of a generation unit is below $2500 \mathrm{~kW}$ with all load models. This maximum can be accepted according to the interconnection policies adopted by many utilities. However, it can exceed the limit adopted by other utilities (see for instance [24]); in such a case, the procedure should be modified to include that limit in calculations. 
To understand these results, it is important to keep in mind that once one unit has been allocated, the location and the rated power of the subsequent units are selected by discarding the nodes to which units previously allocated were connected and taking into account the maximum rated power that results after subtracting the rated power of the allocated generators from the total feeder power.

Figure 8 shows the rated power of the generation units to be connected every year and the cumulative reduction of energy losses, not the yearly reduction, derived from the conventional Monte Carlo method. As expected, the larger values of the rated powers correspond to the first units to be allocated, that is, the rated powers of the unit to be connected at the beginning of year 1 are larger than the rated powers of any unit to be connected in subsequent years, regardless of the load model. However, due to the yearly variation of loads, the rated power of the new PV generator will not be always smaller than any other PV generator in operation, because the energy losses to be compensated for a certain 5-year term could be larger than for a previous term.

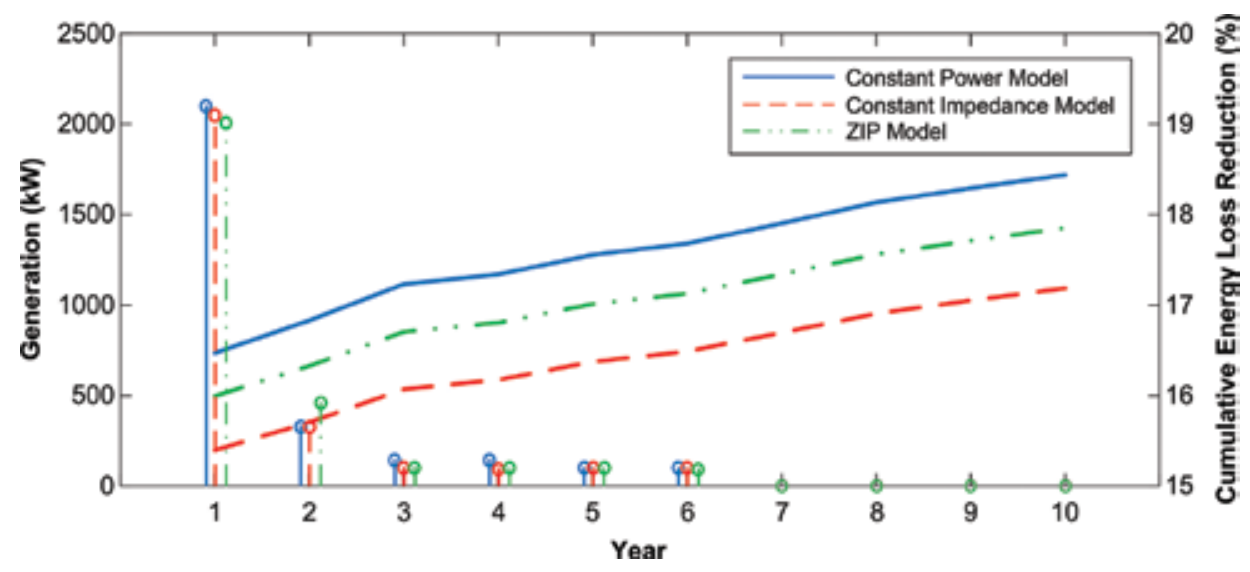

Figure 8. Sequential connection of optimum rated PV generators—conventional Monte Carlo method.

The total power to be allocated is similar for a given model when using either the conventional or the refined method: differences are below $4 \%$. However, differences are larger when comparing the individual rated power values derived from the conventional and the refined methods. A reason for this is discussed in the next section.

As with the short-term evaluation, the highest reduction of energy losses corresponds again to the constant power load model, while the lowest generation is required when the load is represented as constant impedance. As for the reduction of energy losses, the resulting values are different for each load model, and although differences are not too large they cannot be neglected. In addition, the resulting energy losses are basically the same with the two approaches. This supports the conclusion that a quasi-optimum solution can be reached by considering different combinations of locations and rated powers, because the minimum of energy losses is not very sensitive with respect to rated powers and locations of generators. 
From the simulation times presented in Table 8, it is evident that a significant reduction in the CPU (central processing unit) time can be achieved by using the refined approach.

The behavior of the energy loss reduction factor deserves some special attention. Figure 8 shows the energy loss reductions that are obtained at the end of a given year, considering the reduction caused from the beginning of the period. One can note that the total reduction at the end of the studied period is not too large-less than $20 \%$. There are several causes that justify this quantity. Since PV generators inject only active power, energy losses caused by the reactive component of load currents are not compensated. On the other hand, the nature of the solar resource causes that the connected PV generators will not inject power during many hours of the day and the injected power during other hours will be below or far below the maximum value the PV generators can inject. Finally, since the minimization period is 5 years and the load will be higher at the end of this period, the selected rated power will be such that the unit will overcompensate active load during the first years and undercompensate during the last years.

One can also observe that the reduction of energy losses continues after the last PV generator has been connected. Remember that the last unit is connected at the beginning of the sixth year, and its rated power is selected to minimize losses until the end of the tenth year. However, one can also observe that the cumulative energy loss reduction factor continues increasing after the optimization period (i.e., after the year 10).

As proved in [20], the cumulative energy loss reduction factor one year after the optimization period will exceed the cumulative energy loss reduction factor at the end of the optimization period, if the energy loss reduction factor of that year exceeds the cumulative energy loss reduction factor at the end of the period. That is, the cumulative factor will continue increasing while the energy loss reduction factor corresponding to 1 year is larger than the cumulative factor at the end of the previous year. For the test system, the cumulative energy loss reduction factor with a constant power load model at the end of the optimization period is about $18.4 \%$ (see Table 9 and Figure 8), while the energy loss reduction factor during the subsequent year (i.e., year 11 ) is above $18.4 \%$; therefore, if no more generation units are connected, the trend will continue until the year at which the cumulative energy loss reduction factor exceeds the energy loss reduction factor corresponding to that year.

It can be assumed that the reduction of energy losses could be larger if the number of units to be connected was higher; however, the present study is not aimed at estimating the number of units that could achieve the maximum reduction of energy losses. In any case, it is worth mentioning that the reduction of energy losses that can be achieved with more than four units is very small with respect to that achieved with four units [20].

\section{Maximum reduction of energy losses--a systematic approach}

The application of the refined Monte Carlo method has significantly reduced the simulation time, and it has provided energy loss values that are very close to those obtained with the conventional Monte Carlo method. The values were obtained by using $R=5 \%$; see Section 3.6. 
Consider the case when only one PV generation unit has to be allocated. If $R=5 \%$, then the calculations could be carried out by following a systematic method instead of a statistical method, that is, only 20 values should be considered for both the rated power of the generator and the location node. In other words, 400 runs in which values are systematically calculated and combined would be enough to obtain results equal or very similar to those derived from the conventional and the refined methods (see Subsection 3.6).

Note, however, that if the systematic method is applied when two units have to be allocated, then 160,000 (i.e., $20 \times 20 \times 20 \times 20$ ) runs would be respectively required, since four variables must be estimated for each case. Obviously, the systematic method would not be a solution when two or more units are to be allocated. This is in accordance to what was concluded from the results shown in Tables 3 and 4: many more runs than those applied when four units had to be allocated were needed.

The systematic method can be a potential solution for long-term evaluation when PV generation units are sequentially allocated (see Section 4). Tables 10 and $\mathbf{1 1}$ show the results obtained for an evaluation of 10 years when up to six units are sequentially connected.

\begin{tabular}{lllll}
\hline PV generator & $\begin{array}{l}\text { Constant power load } \\
\text { model }\end{array}$ & $\begin{array}{l}\text { Constant impedance load } \\
\text { model }\end{array}$ & $\begin{array}{l}\text { ZIP load } \\
\text { model }\end{array}$ \\
\hline Unit 1 & Node & 375 & 375 & 375 \\
& Rated power $(\mathrm{kW})$ & 2025.0 & 2025.0 & 2025.0 \\
Unit 2 & Node & 225 & 225 & 250 \\
& Rated power $(\mathrm{kW})$ & 517.3 & 388.0 & 388.0 \\
Unit 3 & Node & 500 & 250 & 200 \\
& Rated power $(\mathrm{kW})$ & 107.6 & 114.1 & 228.2 \\
Unit 4 & Node & 250 & 500 & 500 \\
& Rated power $(\mathrm{kW})$ & 108.9 & 115.1 & 109.4 \\
Unit 5 & Node & 200 & 200 & 225 \\
& Rated power $(\mathrm{kW})$ & 107.1 & 112.9 & 107.5 \\
Unit 6 & Node & 475 & 175 & 175 \\
& Rated power $(\mathrm{kW})$ & 104.1 & 109.6 & 104.5 \\
Energy losses $(\mathrm{kWh})$ & 1594536.3 & 1499115.9 & 1544531.8 \\
Simulation & time $(\mathrm{h})$ & 2.14 & & 2.46 \\
\hline
\end{tabular}

Table 10. Long-term evaluation-simplified method.

Some interesting conclusions can be derived from these results:

- Although a systematic procedure (i.e., without generation of random quantities) is applied, the simulation times shown in Table $\mathbf{1 0}$ are longer than those shown in Table $\mathbf{8}$ with the 
refined method. This is due to a simple reason: when using the refined method, together with a value or $R=5 \%$, the total number of runs is less than 400 . For instance, when the load is modeled as a constant power, the number of runs needed to obtain the values shown in Table 8 was respectively 257, 145, 137, 132, 135, and 128.

- The rated powers presented in Table $\mathbf{1 1}$ are larger than those obtained with the refined method (see Table 9); the largest difference between values presented in both tables is more than $9 \%$. However, the reduction in energy losses that is achieved after the 10-year period is basically the same with the refined method and the simplified systematic approach; the largest difference between the energy loss reduction values corresponding to each load is less than $1.5 \%$.

- Although size and location values for the first unit are the same with the three load models, the results derived for the subsequent units exhibit some important discrepancies.

- As with any previous study, the highest reduction of energy losses corresponds to the constant power load model, while the lowest generation is required when the load is represented as constant impedance.

\begin{tabular}{lll}
\hline Load model & Total generation $\mathbf{( k W )}$ & Cumulative energy loss reduction (\%) \\
\hline Constant power & 2970.2 & 18.239 \\
Constant impedance & 2864.9 & 16.857 \\
ZIP & 2962.7 & 17.546 \\
\hline
\end{tabular}

Table 11. Long-term evaluation-total generation and energy loss reduction—simplified method.

A simple conclusion can be derived from this and the previous studies: a refined Monte Carlo method can be accurate enough if the goal is to estimate the minimum energy losses. If the goal includes also an accurate estimation of sizes and locations, then a conventional Monte Carlo method with a good convergence criterion should be applied. Obviously, a simple way to increase the accuracy with size and location values is to decrease the value of $R$; however, as discussed above this can be a too demanding approach if more than one unit is to be allocated.

Although the systematic method and the refined method have both been applied here by setting the Euclidean distance at 5\% without any further justification, the fact is that some simple rules can be used to select a reasonable value and then decide whether any of these approaches is worth applying. Consider the operating conditions of the system studied in this chapter. The maximum power to be allocated cannot exceed the total load (i.e., $4500 \mathrm{~kW}$ ). A $5 \%$ of 4500 is $225 \mathrm{~kW}$, and a $5 \%$ of the power to be allocated after the first unit has been selected is about $125 \mathrm{~kW}$ (i.e., $5 \%$ of about $2475 \mathrm{~kW}$ ). If the rated power of units to be selected is expected to vary in steps of $50 \mathrm{~kW}$, then the $5 \%$ rule is not the adequate one; it should be reduced and the number of runs increased, at least for the first units. A similar reasoning could be followed with the location nodes. If line/cable lengths of the test system are too long, then it would be advisable to reduce the value of $R$. This basically means that the accuracy of the refined and 
the systematic methods can be predicted, and their application is limited by the topology and operating conditions of the test system.

\section{Conclusions}

This chapter has presented a procedure based on a Monte Carlo method for maximum reduction of energy losses using multicore computation. The procedure has been developed to cope with short-term and long-term evaluation periods (i.e., greater than 1 year). The method can be applied to any system regardless of its topology (see [20]). The present procedure is single-objective, but it can be expanded to include other objectives (e.g., minimization of the cost of energy or the cost of interruptions, and system upgrading) when optimizing the allocation of generation units.

Advantages and disadvantages of procedures based on the Monte Carlo method are well known: they are rather simple and can be usually based on advanced models; in turn, they usually require a high number of runs and consequently long CPU times. The application of parallel computing in this work has achieved a significant reduction of the simulation time. The application of multicore environments is becoming a usual practice, and it is foreseen that large multicore systems will be soon available at an affordable cost. In addition, a similar procedure could be implemented in a larger computer cluster (i.e., using grid computing); so, a not-too long simulation time could be achieved without significantly increasing the cost. However, it is evident from the obtained results that the application of a conventional Monte Carlo method to very large distribution systems (i.e., with several thousands of nodes) to which several dozens of DG units are to be connected might not be carried out in an affordable time even if a large multicore installation (i.e., with several hundreds of cores) was used. This can be very obvious when a long-term evaluation is carried out.

The results derived from short-term evaluation show that the energy loss surface is very smooth near the global optimum. The most interesting conclusion is that a quasi-optimum value can be obtained by means of several combinations (i.e., different combinations of node location and rated power of PV generators). That is, values very close to the global minimum (with differences below 1\%) can be reached by considering several results; these findings are the foundation of the refined Monte Carlo approach. Through the application of the 5\% criterion, the number of runs needed with the conventional Monte Carlo method can be significantly reduced without loss of accuracy. The differences between the values derived from different load models are not negligible. This can be seen as a proof of the importance that accurate load models have for this and other studies.

The systematic approach presented in Section 5 can also provide accurate-enough solutions if only minimum energy losses have to be estimated. If the size and locations of the PV generators must be estimated with some accuracy, then the conventional Monte Carlo method should be considered. 
The application of the approaches based on the Monte Carlo method can also be applied to multifeeder distribution systems. Reference [20] proposes a divide-and-conquer approach that can speed up the calculations in multifeeder systems by using also parallel computing.

Some important conclusions are obtained from the comparison between results obtained in this chapter and those presented in [19]. Although the test system was the same in both studies, the results are different. As discussed above, there are several reasons: the energy losses to be minimized in the current study did not include substation losses; the model used for representing PV generation was different in both studies; and there were significant differences between the yearly curves used to represent the time variation of node loads.

It is important to keep in mind that the allocation of distributed generators in actual deregulated systems is not made following a sequential connection of generation units whose sizes are selected to minimize energy losses; in addition, the time at which each unit is connected to the system does not follow any specific pattern (i.e., it can be assumed random). In fact, the procedure implemented for this work can cope with any pattern of DG connections. On the other hand, the long-term evaluation has shown that the maximum reduction of energy losses is achieved by connecting the largest generation unit at the beginning of the period with little room for more energy loss reduction in subsequent years. Remember that for a radial feeder with a uniformly distributed load and an active power demand of $9 \mathrm{~kW}$ at every node, the optimum size of the first unit to be allocated should be more than $2000 \mathrm{~kW}$. The usefulness of this study is in the insight it provides about the impact that the connection of PV generation can have on the system energy losses, that is, utilities can obtain from this or similar studies important information about the maximum loss reduction they should expect.

\section{Author details}

Juan A. Martinez-Velasco* and Gerardo Guerra

*Address all correspondence to: martinez@ee.upc.edu

Polytechnic University of Catalonia, Spain

\section{References}

[1] F.A. Farret and M. Godoy Simões, Integration of Alternative Sources of Energy, John Wiley, Hoboken, NJ. 2006.

[2] T. Ackerman, G. Andersson, and L. Söder, "Distributed generation: A definition," Electr. Power Syst. Res., vol. 57, no. 3, pp. 195-204. 2001.

[3] H. Lee Willis and W.G. Scott, Distributed Power Generation. Planning and Evaluation. Marcel Dekker, New York, NY. 2000. 
[4] R.C. Dugan, R.F. Arritt, T.E. McDermott, S.M. Brahma, and K. Schneider, “Distribution system analysis to support the smart grid," IEEE PES General Meeting, Minneapolis, USA, July 2010.

[5] T. Lambert, P. Gilman, and P. Lilienthal, "Micropower System Modeling with HOMER," Chapter 15 of Integration of Alternative Sources of Energy, F.A. Farret and M. Godoy Simões (Eds.), John Wiley, Hoboken, NJ. 2006.

[6] J.A. Martinez and J. Martin-Arnedo, "Tools for analysis and design of distributed resources - Part I: Tools for feasibility studies," IEEE Trans. Power Deliv., vol. 26, no. 3, pp. 1643-1652, 2011.

[7] R.A. Walling, R. Saint, R.C. Dugan, J. Burke, and L.A. Kojovic, "Summary of distributed resources impact on power delivery systems," IEEE Trans. Power Deliv., vol. 23, no. 3, pp. 1636-1644, 2008.

[8] C. Wang and M.H. Nehrir, "Analytical approaches for optimal placement of distributed generation resources in power systems," IEEE Trans. Power Syst., vol. 19, no. 4, pp. 2068-2076, 2004.

[9] G. Celli, E. Ghaiani, S. Mocci, and F. Pilo, “A multiobjective evolutionary algorithm for the sizing and sitting of distributed generation," IEEE Trans. Power Syst., vol. 20, no. 2, pp. 47-55, 2005.

[10] G. Carpinelli, G. Celli, S. Mocci, F. Pilo, and A. Russo, "Optimization of embedded generation sizing and sitting by using a double trade-off method," IEE Proc. Gener. Trans. Distrib., vol. 152, no. 4, pp. 503-513, 2005.

[11] A. Keane and M. O'Malley, "Optimal allocation of embedded generation on distribution networks," IEEE Trans. Power Syst., vol. 20, no. 3, pp. 1640-1646, 2005.

[12] P.S. Georgilakis and N.D. Hatziargyriou, “Optimal distributed generation placement in power distribution networks: Models, methods, and future research," IEEE Trans. Power Syst., vol. 28, no. 3, pp. 3420-3428, 2013.

[13] P. Prakash and D.K. Khatod, "Optimal sizing and sitting techniques for distributed generation in distribution systems: A review," Renew. Sust. Energy Rev., vol. 57, pp. 111-130, 2016.

[14] Y.G. Hegazy, M.M.A. Salama, and A.Y.Chikhani, "Adequacy assessment of distributed generation systems using Monte Carlo simulation," IEEE Trans. Power Syst., vol. 18, no. 1, pp. 48-52, 2003.

[15] Y.M. Atwa and E.F. El-Saadany, "Probabilistic approach for optimal allocation of windbased distributed generation in distribution systems," IET Renew. Power Gener., vol. 5, no. 1, pp. 79-88, 2011. 
[16] G. Guerra and J.A. Martinez, "A Monte Carlo method for optimum placement of photovoltaic generation using a multicore computing environment," IEEE PES General Meeting, National Harbor, USA, July 2014.

[17] J.A. Martínez-Velasco and G. Guerra, "Analysis of large distribution networks with distributed energy resources," Ingeniare, vol. 23, no. 4, pp. 594-608, 2015.

[18] T. Gönen, Electric Power Distribution System Engineering, 2nd Edition, CRC Press, Boca Raton, FL, 2008.

[19] J.A. Martinez and G. Guerra, "A Parallel Monte Carlo method for optimum allocation of distributed generation," IEEE Trans. Power Syst., vol. 29, no. 6, pp. 2926-2933, 2014.

[20] G. Guerra and J.A. Martinez-Velasco, “Optimum allocation of distributed generation in multi-feeder systems using long term evaluation and assuming voltage-dependent loads," Sust. Energy Grids Netw., vol. 5, pp. 13-26, 2016.

[21] R. Dugan, Reference Guide. The Open Distribution Simulator, EPRI. July 2010.

[22] M. Buehren, MATLAB Library for Parallel Processing on Multiple Cores. Available from http://www.mathworks.com

[23] D.P. Chassin, "Electrical Load Modeling and Simulation," Chapter 10 of High Performance Computing in Power and Energy Systems, S. Kumar Khaitan and A. Gupta (Eds.), Springer, Berlin, Germany. 2013.

[24] The Regulatory Assistance Project, "Interconnection of Distributed Generation to Utility Systems," RAP Report (Main author: P. Sheaffer), September 2011. Available from www.raponline.org 

Chapter 6

\title{
The Role of Middleware in Distributed Energy Systems Integrated in the Smart Grid
}

\author{
Jesús Rodríguez-Molina \\ Additional information is available at the end of the chapter
}

http://dx.doi.org/10.5772/62870

\begin{abstract}
Middleware architectures have proven to be of major importance when dealing with distributed systems, as they are able to abstract the inevitable heterogeneity of the hardware devices present in a deployment with the aim of offering a collection of interfaces and resources of homogeneous appearance to the upper, applicationoriented layers. In an energy-based distributed system as the Smart Grid, this role is replicated, as the hardware devices that are found, while essentially related to the power grid and the functionalities that can be extracted from it (advanced metering infrastructure, remote terminal units, renewable energy resources, etc.), still present the same challenges that other distributed systems are expected to deal with, such as heterogeneous features, different information formats, diversity of their performance procedures, or integration and interconnectivity issues. Therefore, a middleware architecture is still of major usability in the power grid. This chapter offers information about the common features that are present in a middleware architecture that works under the requirements and use cases typical of the Smart Grid, as well as offers examples on how middleware integrates legacy, proprietary, and newly developed pieces of equipment within the same distributed energy grid.
\end{abstract}

Keywords: middleware, distributed systems, software architecture, data management, service integration

\section{Introduction}

Interconnecting hardware devices of different features has been a challenge faced repeated times in different areas of knowledge. It started as a task to be doneat the network layer, where different proprietary systems struggled to communicate with each other when equipment from differ- 
ent manufacturers had to cooperate in retrieving and exchanging information. Some of the most prominent organizations regarding standardization (ISO, CCITT) started working back in the late 1970s [1] in a layered solution that would allow interoperability among different pieces of equipment while (open system interconnection (OSI) model), around the same time, another layered architecture would impose its own criteria in order to solve interconnectivity challenges (transmission control protocol/Internet protocol (TCP/IP) model). While those solutionsespecially the latter one, which became widely adopted in the following years-offered reasonably accurate patterns on what could be obtained from each of the layers (implementation details wereleft toeach of themanufacturers, which only had to guarantee that their products would be compliant with the specifications described in each of the layers), few holistic implementations were offered upper than the network layer. This fact implied that despite the network and lower layers had standardized protocols that would accomplish the expected functionalities for connection-oriented and connectionless communications, solving the challenges associated with the different formats for data representation in upper levels (as session or presentation in the OSI model, and most of the application layer in TCP/IP) would stillbea major challenge, asincreasingly differing devices werebeing used in distributed systems. As far as the Smart Grid is concerned, each of the microgrids that are involved in the larger deployment might count with appliances that have little to do with the ones that are found in another one. For example, advanced metering infrastructure may provide different services and be developed by a different company in one microgrid if compared to another one; even the same one is likely to have pieces of equipment from different vendors. Advanced end users or developers with enough know-how are willing to create their own smart meters by integrating hardware and software solutions reliant on open source technologies to provide services, thus adding a degree on unpredictability in the information formats that are used throughout the Smart Grid. If some other devices and/or resources are taken into account (RTUs, DERs, RESs, etc.), the differences among the infrastructures and hardware components grow exponentially. Clearly, a solution that goes beyond purely network-based communications, which are oblivious to the meaning of the information that is interchanged (only network datagrams are considered, thusleaving the data unprocessed and without knowledgeinference), is required for upper layers.

Fortunately, middleware can be used to solve these issues. Middleware can be defined as a software-based layer located between the lower, network- and hardware-based layers and the higher, application-based ones, which has as its main functionality the abstraction of the underlying heterogeneity associated with the different hardware devices participating in a distributed deployment, in a way that a collection of homogeneous-looking interfaces (more specifically, a set of application programming interfaces or APIs) will be offered to the end users of the application layer. The main functionality of middleware becomes evident in Figure 1: despite the different data formats used by the devices located in different levels, the middleware layer still homogenizes the collected data from lower ones, withholding the underlying heterogeneity of the whole system.

When all is said and done, middleware architectures are used in heterogeneous, noncentralized systems where data collection is scattered throughout a collection of different pieces of 


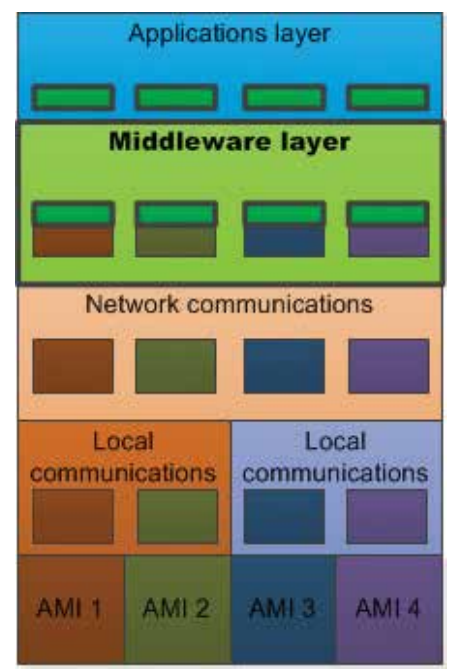

Figure 1. Generic middleware architecture for the Smart Grid.

equipment responsible for harvesting data, transferring them and either taking decisions or executing commands made by human beings. When considering how energy production, transport, and consumption can be managed, it becomes clear that if it is done in a distributed manner and using the infrastructure of a smart grid, middleware can be very convenient for a plethora of tasks:

1. A middleware architecture in a distributed system effectively acts as the "glue" that puts together many different hardware and software entities. It processes the data in a way that it will be offered to users and applications (human operators, mobile apps, etc.) oblivious to the underlying complexity and different appliances used in the distributed energy generation systems.

2. A middleware architecture can be used to enhance the available services developed for the system. If, for example, load balance, context awareness, real-time pricing, security, semantic capabilities or data aggregation functionalities are desired, they can be added as part of the middleware architecture in case they cannot be installed either as part of the hardware or the applications. This is a situation that happens more often than not, as applications may be too lightweight to be able to perform calculations expected from different features (statistic information, big data management) and hardware devices might be either not powerful enough to perform those calculations, or may be proprietary and/or legacy devices that cannot be reprogrammed, or else they would be regarded as "hacked" by their vendors.

3. A middleware architecture allows the creation or enhancement of business models, as it can be used under different kinds of service paradigms, such as Software-as-a-Service or Platform-as-a-Service. In addition to that, application developers can have a very easy time connecting to the middleware architecture their own pieces of work, as they only need to care about the upper level interfaces that the middleware architecture offers them. 
4. Prosumers can manage the amount of energy in a more flexible manner, as they are able to use the services that are in the middleware to decide how much or when to pour their produced electricity into the power grid, they can coordinate with other users to offer a better price for the energy, etc.

There are several parameters that must be considered when a middleware architecture is to be developed for a system:

a. How transferred information is going to be homogenized: the data transferred by different devices located at the last mile of the distributed system may be made by different manufacturers that use their own proprietary data format. Due to that, it becomes even more evident that middleware is useful to solve this kind of challenges. However, it has to be figured out the way that data formats will be the same for all the existing devices. One of the ways that can be used to solve that issue is by using semantic resources as common information model or CIM [2]. CIM is a standard somewhat resembling unified modeling language that can be used as a way to design software in a smart grid-based system. In addition to that, CIM can be extended by additions done in a microgrid, so they will be added to the overall available libraries. An architecture based on CIM relies heavily on semantics, which can also be used as a way to create or extend existing ontologies that will not only expand the collection of terms and associations among those terms, but also add more capabilities to the middleware using them, as the capacity of inferring knowledge from information or even raw data will be of major importance for taking decisions.

b. Functional and nonfunctional requirements that are to be born in mind when designing the middleware architecture: functional requirements will be turned into use cases that represent the functionalities expected from a distributed, energy-based system. These requirements are conceived to improve the existing state of the art at micro- and macroservices level: on the one hand, already present services must be improved in terms of efficiency (doing the same task faster) and complexity (more complicated tasks can be performed as well). On the other hand, new services can be included in the middleware architecture that can be based on purely software capabilities (context awareness, semantic capabilities, publish/subscribe paradigm, security) or more involved in distributed energy generation (load balance, demand side management, tariff calculation).

c. Present and already functioning models must be studied as well. One of the most widely known is the smart grid architecture model or SGAM [3], where different layers (component, communication, information, function, business) are responsible for different attributes that model a whole Smart Grid system with a holistic point of view. While there is a considerable room for improvement and innovation, this and more models and proposals can be used as a starting point for further studies and developments.

Overall, research activities done to develop the middleware architecture must consider the current state of the art to have a good grasp of the status of the existing solutions (especially regarding the open issues and challenges that remain to be dealt with), improving it with functional and nonfunctional requirements, either being inferred from the state of the art or motivated by a research project, and obtaining use cases from those requirements, which will 
result in software modules that satisfy them. Special care has also to be taken to how information will become standardized in the system, as well as the possible constrains that are introduced by nonfunctional requirements. The resulting output will be a middleware architecture that will be compliant with previously formulated objectives: it will be distributed, enhanced with several software modules and capable to manage energy generation and distribution. Typically, it will result in a layered architecture that will handle hardware and application information at the lower and higher borders, whereas there will be a core area with all the services required for data management and distributed energy functionalities.

This chapter is devoted to the definition of middleware architectures for distributed systems and how they can be used in an environment related to distributed generation of energy, such as the smart grid. The main features of middleware will be displayed, as well as a methodology to do research on this topic, and a collection of services design that can be used as a templated for future implementation works.

\section{Methodology for middleware design in the Smart Grid}

When considering the requirements to build a middleware architecture focused on the functionalities to be made use of in the Smart Grid, there are some facts that must be observed. Since research activities should be used here as a tool to build a commercial, usable solution that will effectively improve the already existing power grid, three lines of work will be followed, that is to say, the state of the art regarding existing developments, functional and nonfunctional requirements and the homogenization procedures that will be carried out for the main functionalities of the middleware. Following each of these, three lines will have different impacts on the methodology, as each of them deals with a different side of the

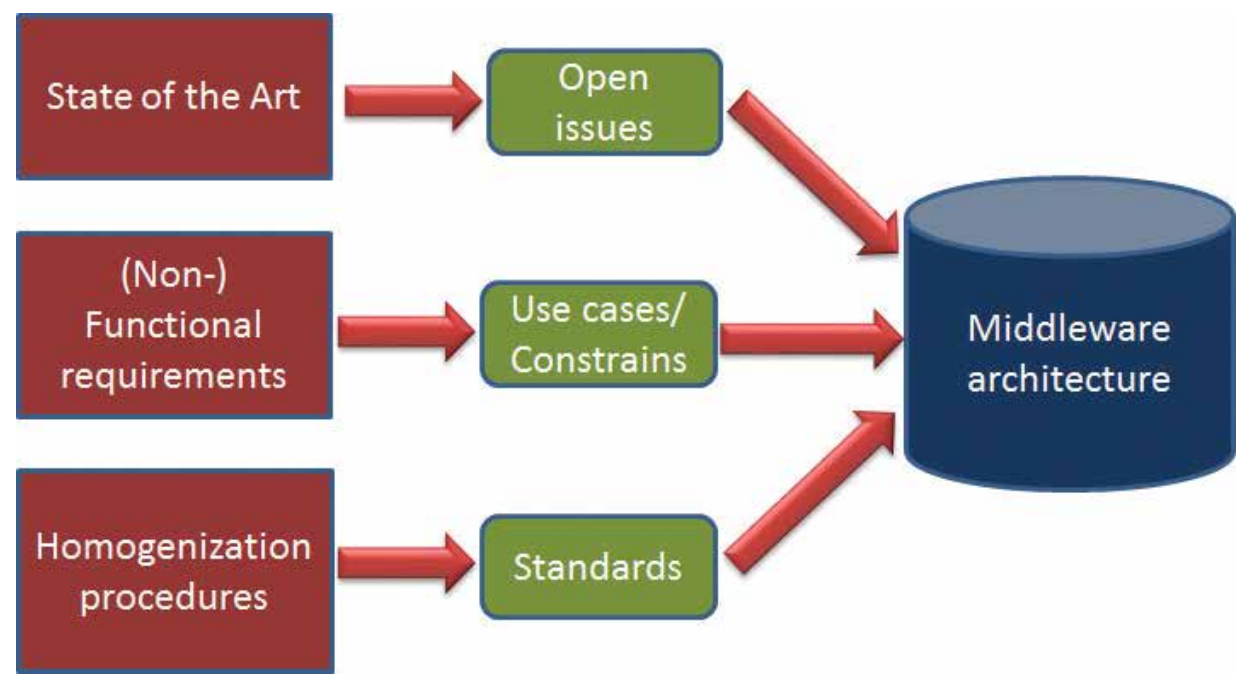

Figure 2. Middleware location and main functionality. 
challenges for designing the architecture. For example, the study on the state of the art will result in acquiring knowledge not only regarding the existing solutions, but also their most usual strengths and weaknesses. Similarly, functional and nonfunctional requirements will imply the elaboration of a plethora of use cases that will result in a sequence of actions that will have their usability established by the nonfunctional requirements defining the system (more specifically, nonfunctional requirements will describe how the middleware architecture present in the Smart Grid will be and functional requirements will depict what that middleware architecture is capable of doing). Finally, homogenization solutions define what kind of data models are going to be used for information representation once the processed data are handled to the upper layers of the deployment. The synergy of these three lines of research will result in a middleware architecture suitable for the Smart Grid, as depicted in Figure 2.

The different software components and features will be a matter to be described in the next chapter, once the procedures to design a middleware architecture for the Smart Grid are fully understood.

\subsection{Study of the state of the art in middleware architectures}

The study of the already available solutions regarding middleware architectures for the Smart Grid should come as the first step to be done when developing a new one. The logic behind this reasoning is getting a grasp of the features and possibilities of the already developed works, as it is more cost-efficient in terms of monetary and time resources counting with some previous point to start the actual development, rather than having to "reinvent the wheel" every time a new architecture has to be designed and implemented. Depending on the needs of one deployment, the capabilities of one solution might be enough for the requirements of the project, albeit it should be considered as a symptom of having not so original work or research objectives. Besides, it has to be taken into account that some of the solutions might have not been disseminated enough, and in fact they could be only partial implementations of theoretical proposals, with little to no code available to be reused or at least be employed as a template for a development done from scratch. The tools that can be used to get a grasp on the available solutions are as follows:

- Scientific papers: these are one of the most common know-how resources that can be used to have an idea of the work carried out in a particular area of knowledge. Typically, it is best to use a scientific-themed search engine in order to look for scientific papers, so that a collection of them will be composed and they can be consulted in search for relevant information. Relevant search engines with scientific papers to show are Google Scholar [4] or the IEEE Xplorer Digital Library [5]. It is not rare that some of these search engines have their full load of content only accessible under a subscription, where a monthly fee has to be paid to access to all the data. Nevertheless, some institutions (research centers, universities, etc.) pay for that service and offer it in a transparent manner to their own members (research staff, students, etc.). Several academic publishing companies may also have a scientific search engine in their websites. As far as information collection from other purposes is concerned, there are two kind of scientific papers that can be taken into account, which can be categorized as survey-themed and innovation-themed papers. Usually, 
survey-themed papers have already collected the state of the art of a particular topic that could match with a varying degree of accuracy the one that the researchers are aiming their efforts to. They can be expected to contain a list of solutions already published and tested depending on the location of their area of interest (e.g., theoretical proposals may have been tested in a laboratory or in a research institution, whereas project-related ones might have to prove the exploitability of their concepts from a more business-oriented point of view, or have to be deployed during a long period of time). Innovation-themed papers are more focused on describing a novel idea that implies a step forward in the boundaries of knowledge on a certain topic. These latter ones often contain a Related Works section where available solutions (at the time of having the innovation-themed paper published) are reviewed to an extent. Experienced readers of scientific papers are usually capable of distinguishing which content or kind of paper is more interesting for their goals.

- Online and offline literature: One of the most obvious ways to obtain information from a topic is consulting books on it. These resources might be found both as online (eBooks, online journals) and offline resources (hardcopy books and journals). Academic publishers and journals are one of the longest existing sources of relevant information in research for different areas of knowledge. It is not uncommon to find out that some of them started publishing scientific works during the nineteenth century [6]. As far as middleware is concerned, The Complete Book of Middleware [7] or Middleware and Enterprise Application Integration [8] could be regarded as some of the most popular books due to the accuracy and detail of their content. The Smart Grid also has its own share of advisable literature, with journals as IEEE [9] offering profuse data about how the power grid can be made smart. Special issues from scientific journals can be used as a source of knowledge as well, especially if they match the topic of interest that is being researched. It must be taken into account, though, that while there are journals devoted to very specific areas of knowledge there is no one, to the best of the author's knowledge, which is fully focused on middleware architectures. Furthermore, white papers (that can be defined as reports of short length that attempt to define clear features about a specific issue regarding Information and Communication Technologies) can also be used as a source of knowledge. Both the Smart Grid [10] and some commercial distributions of middleware [11] have several white papers providing knowledge, which provide a very immediate source of information that can be used by technicians or staff with a lower degree of interest in research.

- Research Projects: Research projects funded all around the world by public national or supranational authorities [12,13], semiprivate or private research institutions or universities usually offer some public resources, as part of the tasks that have to be done regarding dissemination and documentation of the very project that is being funded. The easiest way to obtain material from research projects is checking their own websites [14, 15], although members of the consortium made by all the partners participating in the project can be contacted as well, provided that they offer contact details. The available online materials that can be downloaded from the websites of those projects can be very diverse; the most usual ones include public deliverables (documents with features of different parts or tasks from the project that describe the development tasks that have been carried out), an index 
of the publications associated with the project (showing the name of the publication, its authors and the journal where it was accepted) and the partners that are included in the consortium responsible for the execution of the project.

- Repositories and hosting services: as far as implementation works are concerned, a possible starting point to create a new software solution can be obtaining feedback from the online available code in order to get an idea on how to create a software development, design software functionalities or even debug some already developed code. The most widely known repository of these characteristics is GitHub [16], where code that is willingly uploaded by a large number of developers can be obtained, improved and shared again, as long as the person interested in obtaining and sharing data creates an account in the site. Although repositories can be a useful place to get some immediate knowledge on a specific task, the code that is shown is usually provided "as is," without any guarantee of it working flawlessly (in the end, it makes sense that is done like that, as it is obtained cost-free) or completely fulfilling the tasks it was expected to perform.

- Social networks: There are some scientific research social networks that can also be used as a way to collect information from the current state of the art in one area of knowledge, as the members of those networks can be scientists with a high level of expertise [17] able to provide some background information. As it happens with repositories and hosting services, scientific social networks must be approached with a degree of caution, since reliability of social networks as a way to obtain feedback is usually way lower than the one obtained from more orthodox sources, as the quantity and quality of the answers relies on quantity and quality of the peers willing to provide their know-how in one topic. It is not uncommon to obtain misleading or plainly wrong answers to a question that will hinder the undergoing research. Social networks should be used in close cooperation-and subordination-to other more scientifically sound sources rather than being taken as the only source of research material.

\subsection{Election of functional and nonfunctional requirements}

From the resource usage point of view, the requirements for a Smart Grid middleware architecture may vary from one deployment to another, but in the end, they result similar in most of the cases. Functional requirements are strongly related to the functionalities that are expected from a system of these features. They involve a certain number of capabilities that the system (by system, it is meant the microgrid that is deployed as part of a wider Smart Grid) offers to some actors, who may or may not be human beings (machinery located outside the Smart Grid, e.g., may make use of services offered by it, such as tariff policy elaboration and modification in real time).

On the other hand, nonfunctional requirements are very specific features that are imposed on the system, which will have an impact in system performance (by setting a minimum level of certain parameters or limiting the capabilities of the system). Rather than describing or being related to system functionalities, they set the boundaries for them, so that the functional requirements will be fulfilled with success within the borders set by the nonfunctional ones. The reasons for having strict nonfunctional requirements are varied and are usually due to 
technical (hardware capabilities of the devices used in the microgrid, open or proprietary hardware, location of the required appliances in a deployment) or economical (budget limits, Service Level Agreements, reutilization of already existing premises and/or equipment) motivations.

\subsubsection{Functional requirements}

When actors become engaged to the functional requirements the latter start being referred to as use cases. According to the principles of software engineering, use cases can be defined as a group of events and actions performed within the boundaries of a system for the benefit of some actors out of it, but using the system. In this context, functional requirements define those events and actions to be made by the power grid once it has been enhanced with a middleware architecture, so the latter is pivotal to obtain the desired performance from the former. A few of the most common use cases are as follows:

- Device registration: When one new device is added to the microgrid, its existence must be acknowledged by the whole system in order to obtain and provide data used to perform the functionalities described by the functional requirements. The most usual new equipment to be added are smart meters, as the last mile infrastructure of a microgrid integrated in the Smart Grid is more likely to change than the core of equipment and technologies (due to client rotation, more frequent smart meter substitution due to low prices, etc.).

- Data request: As in any distributed system, data will be offered as a service for the end users at the peripheral zone of the system. For them, data will be of critical importance in order to make decisions regarding their energy consumption or the services they would like to obtain from Smart Grid stakeholders such as the transmission system operator (TSO) or even the distribution system operator (DSO), in case they count with their own supply of renewable energy systems (RESs). Data can be requested with end user intervention (information for end-user mobile apps or web applications in a more general) or without it (bidirectional data transfers between advanced metering infrastructure and the core of the Smart Grid).

- Alarm triggering: Alarms might be triggered in the microgrid if an unforeseen event takes place; they can be related to some kind of economic feature (tariff limits, overspending, waste of energy) or a physical one (damage in one piece of the installed equipment, software failures). As it can be guessed, alarms are usually triggered by either the software installed in the distributed pieces of equipment or by the equipment themselves, rather than by any end user (unless they have been given the option to do so by means of an application).

These use cases can be expressed in a more formalized manner by means of unified modeling language (UML). As it is implied by its name, UML is a software modeling language that is profusely used for software design as a previous step to implementation works [18]. Since one of its main goals is describing in a graphical and accurate manner the features of one system, as well as the relationships among its different components, it comes in handy for the representation of the uses cases that will be studied at the middleware architecture level. UML makes use of many different diagrams to explain the different functionalities and viewpoints 
of one system; for example, computational analysis can be used to have a reference of the different subsystems that are used in the overall middleware architecture, along with the relationships that are held among them. Thus, subsystem diagrams are used in order to depict those relations and subsystems. Additionally, functional analysis is used to describe some other features of the system related with the functional requirements: it is here where the use case diagrams that show the defined use cases, along with their links to the actor taking part of the system as external stakeholders, are represented. Use case diagrams are employed to describe elements of the Smart Grid (as it is shown in the Figure 3), display the actors involved in the system, the use cases that have been contemplated, the boundaries of the system containing those use cases and the relations between use cases and actors. While the content of the use cases might differ greatly from one are of knowledge from other (a microgrid is very different from a wireless sensor network or a group of cooperating robots), their representation varies little among them.

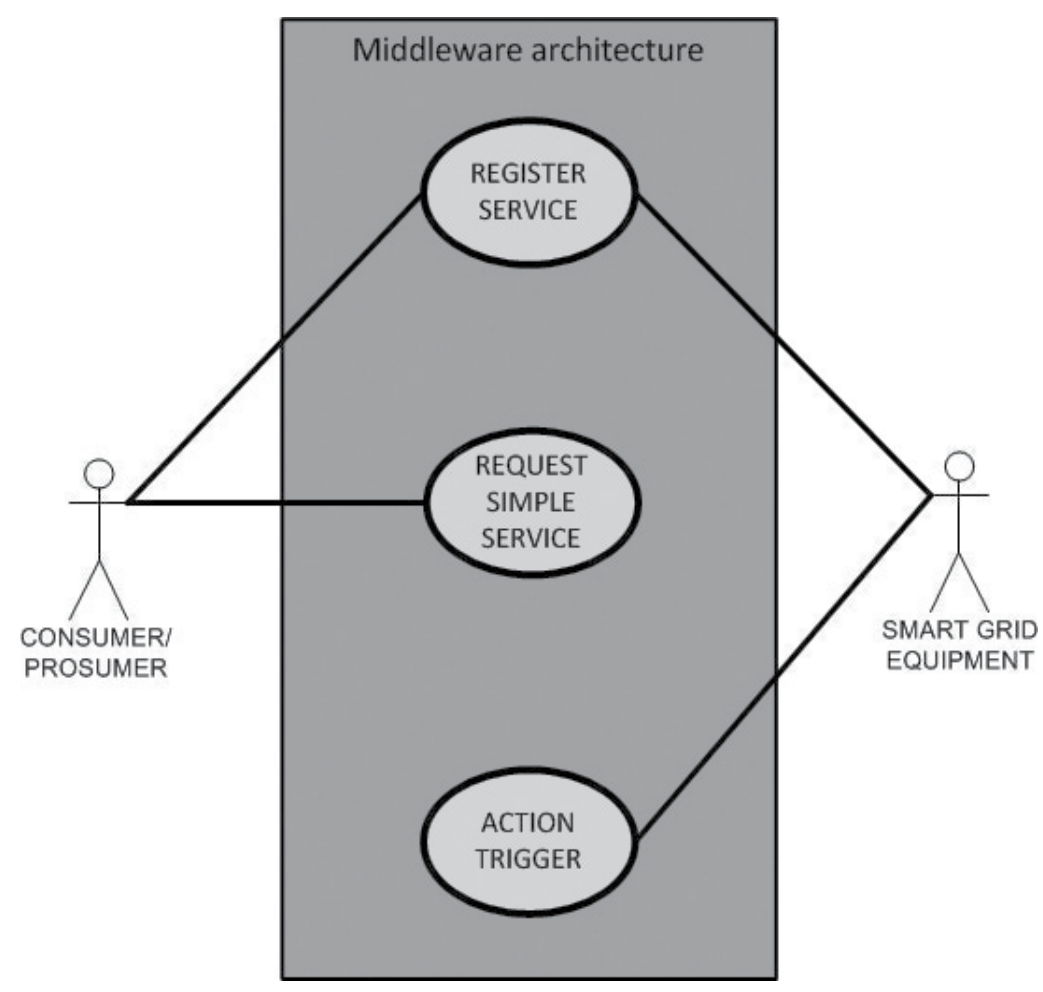

Figure 3. Use case diagram for common use cases.

At the same time, component diagrams depict the different software elements that make a subsystem and how they obtain resources one from the other by means of component interfaces that transfer the information among the existing components. Some other diagrams are advised to be used for functional analysis; for example, sequence diagrams show the set of 
steps that are made for a use case to be completed. Each of those steps is a software instance that is needed to get through to complete the flow of actions that will end up providing the service, as each of them provides a significant inner action for the fulfillment of the requirement defined by the use case. Sequence diagrams must be interpreted in a bi-dimensional fashion: the horizontal axis represents the direction of the information exchange (which uses the software instances and the name given to the actions that communicate one instance with the other in both directions), whereas the vertical one represents the time that is used by each of the software entities in the flow of the represented use case. Last but not least, those software entities and action names are better represented by the usage of class diagrams, which gather the different actions to be taken as methods or functions within the software instances that were used in the sequence diagram as instances. Class diagrams are usually very close to the implementation stage, which is typically carried out after the classes and methods defined by this diagram become definitive. An example of sequence diagrams for the already studied use cases is shown in Figure 4.

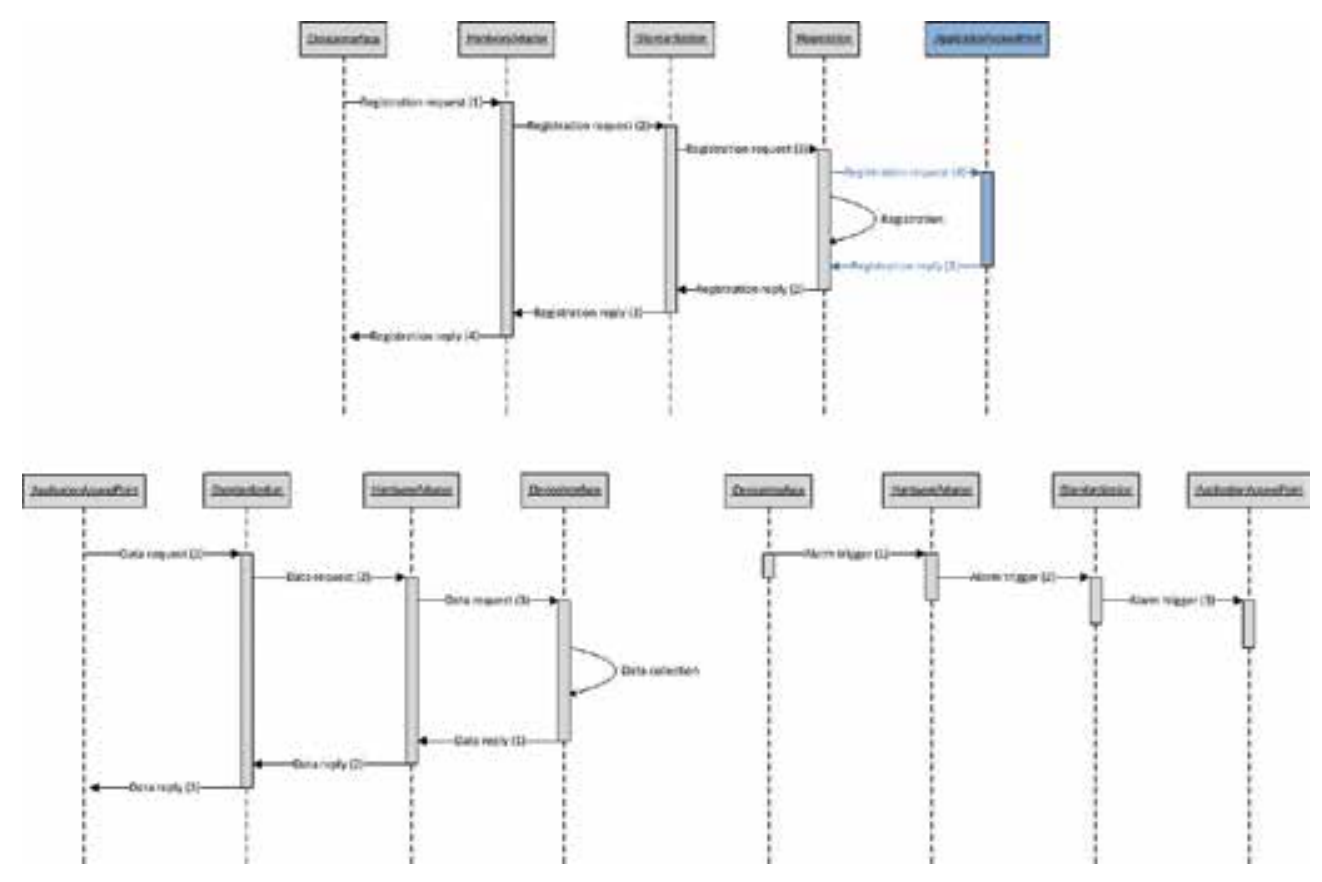

Figure 4. Sequence diagrams for the previously shown use cases.

Here, it can be seen in the first sequence diagram how a typical device registration procedure would take place: the interface of the device that is going to be registered keeps the hardware in touch with the communication services enabled in the Smart Grid. By means of this interface, a first request message is sent. This message is transferred throughout the communications system (which will typically involve IP communications at the network level and TCP/UDP at the transport one) until it reaches the middleware component responsible for receiving 
request messages from devices (hardware abstractor). This component will send the received message to the software module in charge of formatting the messages delivered from levels located lower than the middleware layer (standardization). This is the message, formatted according to what has been defined for the middleware (and containing all the information regarding the services that can be offered by the device that is going to be registered) that will be used for registration. Commonly, a registration acknowledgement message will be sent as a reply to the device that requested the registration and data transfer will be ready to be done, although depending on the degree of control that is given to the end user, the registration request may be sent to the application access point to be evaluated by a human operator. The other two sequence diagrams deal with data interchanges and alarm triggering. Data are requested from an application connected to the middleware via the application access point and is sent though the standardization module in order to have it sent according to the format that is understandable by the hardware device that is being requested. Hardware abstractor sends the request through the network layer until it is received by the interface at the very device capable of providing the service. Once the data are collected, they can be sent back to the middleware architecture by means of the communications network until they are received again at the application access point, which will interface with the application to deliver the data. Alarm triggering is a process used to inform the end user about an abnormal situation that has happened in the grid. Depending on the nature of the alarm, it might be sent by either the hardware component that has detected the issue or other system entities, such as a middleware module. When the issue is detected by a hardware device (as depicted in Figure 4), a message containing information about it will be sent through the communication system to the middleware components previously depicted (hardware abstractor, standardization) until it reaches the application access point, which will send it the application as an alarm that has been push from the underlying system.

As far as the Smart Grid is concerned, the boundaries of those use cases are frequently defined by the most hardware-based elements of the deployment (power grid appliances, smart meters, etc.) on one side, and the closest software entity possible to an end user (mobile app, website, etc.). Hence, the sequence diagrams are representing the relations among those border elements by means of action interchanges among some other, more internal software instances.

Another very accurate representation of the different stages used to design a middleware architecture can be found in [19]. Here, it is explained how the different functionalities and software modules are conceived, related and located in the middleware system.

\subsubsection{Nonfunctional requirements}

The other kind of requirements that was presented is the nonfunctional ones. As it happened with the former, they are present in almost any imaginable distributed system, although they can appear in any other system that is not distributed, as long as some boundaries are imposed to have a system working under realistic circumstances. Nonfunctional requirements are a group of features that strictly define the main features that establish what a system is. This is something that must be born in mind, as sometimes they can be loosely interpreted as the 
boundaries of one system. Nonfunctional requirements have been used for quite a while in the design of both hardware and software products. For example, nonfunctional requirements established during the design stages of the popular Citroën $2 \mathrm{CV}$ where used to devise "a design brief for a low-priced, rugged "umbrella on four wheels" that would enable four small farmers / peasants to drive $50 \mathrm{~kg}(110 \mathrm{lb})$ of farm goods to market at $50 \mathrm{~km} / \mathrm{h}(31 \mathrm{mph})$ " [20]. By defining what that car would be, the boundaries of that design were implicitly mentioned as well. It is easy to regard nonfunctional requirements as constrains; however, they usually describe features that are necessary to understand the system that is being design, rather than imposing mutilating conditions to the system.

As for the middleware architectures for the Smart Grid, nonfunctional requirements are often focused on the properties that both distributed systems and the power grid have: on the one hand, features as scalability, resilience or data security impact the system from a distributed software development point of view. On the other hand, electricity distribution characteristics as power consumption or load peak limits have their own impact in the design of the overall system too.

\subsection{Semantics and middleware architectures}

As previously stated, the main reason for middleware architecture to exist is its capacity to homogenize the different information representation formats that are received from lower layer elements. Among the several software tools that can be used to carry out this functionality, semantics is one of the most effective. In information and communication technologies, semantics can be defined as the capability of a system to infer knowledge form gathered data so that the meaning of the transmitted data will be apprehended by it and the system will be able to use it in the future to optimize its overall performance. The core idea around semantics is that raw data are not only meant to be transmitted from one remote location to another but also processed in a way that will make the system able to infer the actual meaning of the information that is being transmitted throughout the distributed system. For example, should raw data be transmitted about temperature in an equipment, a semantically enhanced system will be able to both transfer the data and assess them, so that an educated decision can be taken with regards of the information transmitted. In a nutshell, a middleware architecture using semantics will have learnt, or inferred, that a very higher than usual temperature means that there is some kind of trouble with the appliance or the geographical area the temperature reading was retrieved from.

Semantics become implemented in a more tangible way by means of ontologies. An ontology is a collection of terms and definitions of wide use in a software system (which may be distributed or not, but it is assumed here that it will be so, as the rules of ontologies also apply to them), along with the relationships between those terms, to the point that it should be considered more as a graph with interrelated elements rather than a dictionary [21]. In addition to that, an ontology can be updated to incorporate new elements or even drop deprecated ones, 
so the knowledge that is acquired by the system can be refreshed as it works during its usual lifespan, thus becoming "wiser" as time goes on.

As described in [19], a semantic module usually consists of several parts:

- An action collector that will retrieve the actions that are being carried out by the system. The pace for collecting actions data can be adapted to the needs of the system, ranging from several times during the day to an almost real-time pace. The smaller of that time span the work strain is put to the whole module (and by proxy, the middleware architecture), though.

- An inference managing entity that infers knowledge for the system, based on the actions that have been collected, the rules fixed for the system and another repository for stored facts.

- A rules repository that stores the norms that have been fixed for the system. They are of major importance to decide whether an action should be triggered or not.

- A facts repository where the past actions that were gathered by the action collector are kept.

- An action trigger component that will be used to send commands whenever the semantic module rules it is necessary. This component comes especially in handy for managing alarms and events.

The most popular semantics element used in the Smart Grid is the CIM [2], which defines its own ontology for most of the pieces of equipment that can be found in a power grid. The representation of CIM is identical to what can be expected from a UML one; this can be deemed as a token of the increasing involvement of software engineering in the power grid.

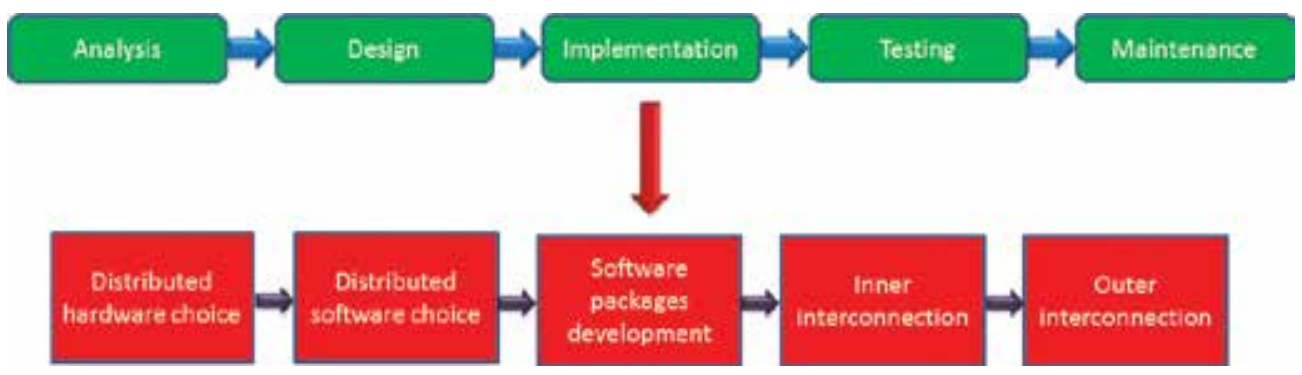

Figure 5. Waterfall model for software design and development tasks for middleware.

Overall, the methodology used to design a middleware for the Smart Grid may be flexible to an extent. For example, the traditional waterfall model can be used to complete the intermediation layer until all its expected functionalities have been performed (see Figure 5), even though it is advisable to have some feedback in each of the stages in the very likely case that any bug or deviation from the scheduled functionalities has to be dealt with. Usually, when 
tackling the implementation stage, there are five steps that must be taken into account to successfully complete the development works of the middleware architecture:

- A decision must be made regarding which hardware devices present in the Smart Grid deployment will be used to have the middleware installed. There might be some appliances that are proprietary solutions without any possibility to have new software installed.

- The software platform that is going to be used to have all the software components installed must also be chosen. It should be capable to work in a distributed way, sending messages from one part of the platform to other ones located remotely. Enterprise service bus architectures (ESBs) work in a suitable manner to solve this challenge, and they can send messages among different software components embedded in them.

- The software packages that are developed to implement the services that are going to be located in the middleware architecture must be codified once the hardware devices and the software platform have been defined.

- Connectivity among the software modules that have been implemented must be guaranteed in order to have them all able to send and receive information whenever there are data to be transmitted through the middleware architecture, either toward the application layer being used by the last mile clients or the network layer interconnecting the deployed appliances.

- Connectivity among the different elements outside the middleware must be accomplished so that there will be a seamless integration of all the subsystems that conform the Smart Grid (or at least, the microgrid where they have been deployed) and data can be transferred from/ to the applications developed on top of the middleware architecture to/from the hardware devices present in the system deployment done.

\section{Services and components in a layered middleware architecture}

The deployments where middleware architectures are used have some important similarities, as it has already been hinted in several sections. These can be easily obtained by taking into account the most usual functional requirements that are expected from those deployments, that is, (a) hardware abstraction for communications between the intermediate software layer and the pieces of equipment in the Smart Grid, (b) semantic capabilities to make the overall system smarter, (c) upper-level access points to the applications that are interconnected to the middleware, (d) registration of the detected devices, (e) other services as context awareness, security, etc. Arguably, wherever a middleware architecture becomes deployed, those services are taken for granted by the end user, who will count on them without noticing which software or hardware entity is providing them. Taking that fact into account, a generic middleware layer can be defined that will be matching most of the requirements that have been imposed to the system. The appearance of the architecture is displayed in Figure 6: 

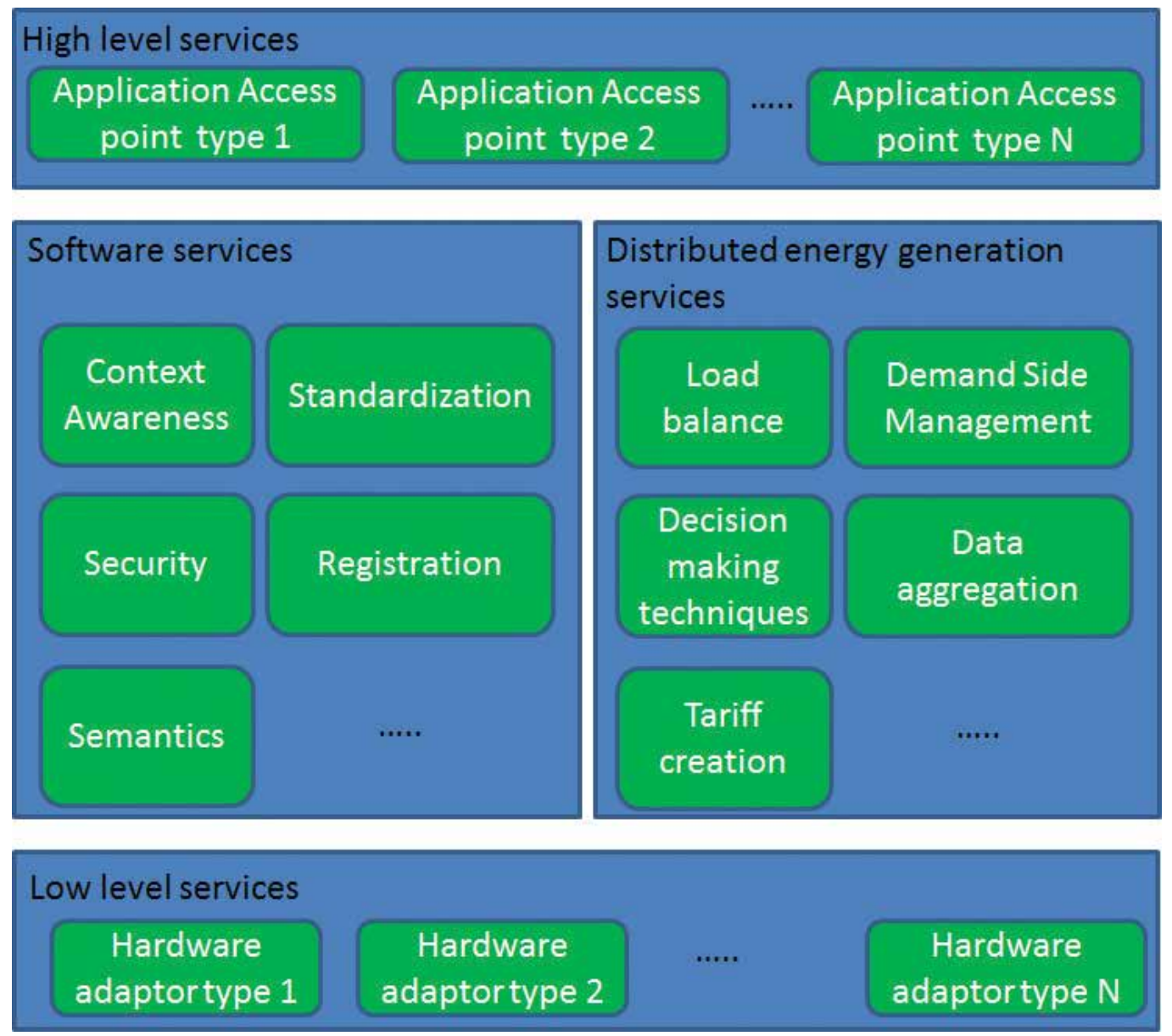

Figure 6. Waterfall model for software design and development tasks for middleware.

The services that are going to be offered have been divided into four different kinds, each of them composing groups of services that are located in different places according to their characteristics. They are as follows:

- Low-level services: These are the ones that are more closely interacting with the pieces of equipment where the middleware architecture has been deployed. Basically, they are responsible for receiving the data that is transferred from the interfaces of the appliances that are used in a microgrid. Consequently, definition of the behavior of the low-level interfaces between middleware and hardware is critical for the correct delivery of the information. There are several protocols that can be used for that matter. For example, Java Message Service (JMS) [22] can be used between entities capable of understanding that programming language. Another protocol suitable for this task is advanced message queuing protocol (AMQP) [23] which is able to communicate devices by means of message queuing. The information interchange will be performed by using hardware adaptor 
modules, which will change their codification depending on the nature of the devise that are interfaced.

- Distributed energy generation services: These are the services that are most closely related to the Smart Grid capabilities. Furthermore, this is the module that is most prone to changes, as the services that are required from one microgrid deployment to another one (let alone a completely different distributed system) may differ greatly. The most usual services that can be found are for load balance (defining the shared amount of resources that each of the elements present in the grid is going to cooperate with to deliver energy), making decisions (regarding both technical and management issues), creating tariffs (with the purpose of offering them in a dynamic way, as a mobile phone contract or ASDL services), aggregating data (in order to collect statistical information about the transferred information that will optimize other services, such as load balance or processes involved in making decisions) or offering demand side management (as a way to shave the power peaks that happen during certain time spans).

- Software services: They offer a significant amount of focus in the distributed system nature of the deployment. They can be found in almost any environment that implies a middleware architecture used as an intermediation layer among devices. The most important ones are context awareness (in order to notice any change in the deployed elements and having the middleware reacting to those changes in cooperation with the semantic operations), standardization of the transferred information, security (which might be offered as encryption services in this group of services or as secure web interfaces in the high-level services), registration (so as to have a collection of the available devices and the services they can provide to the end users or other elements of the Smart Grid) and semantics (working closely with standardization to use a common data model throughout the whole middleware architecture).

- High-level services: The weight of these services in the architecture may vary from one deployment to the other: in some cases, they behave as if they were applications, and in some other, they just offer an access point to the actual applications. Among the technologies that can be used to implement the interfaces for information exchange from/to the applications are constrained application protocol (CoAP) [24], useful for lightweight requirements, and representational state transfer (REST) interfaces [25], which offer an easy way to establish communications via web services.

The most usual way for them to be using each of the functionalities is expressed in Figure 7. As it can be seen, the subsystems need functionalities from each other, namely, the high-level services subsystem is using the software and distributed energy subsystems to obtain the services contained in each of those parts of the middleware. At the same time, those two subsystems are interacting with the low-level services to collect the information fetched from outer, lower layers. Interestingly enough, software services are often used by the services that are more typical of the smart grid, but they are use more as a way to offer support than providing an actual service to the end user, so software services rarely employ the distributed energy services (hence the unidirectional arrow in Figure 7). In any case, depending on the use case that is being dealt with, some components of the middleware architecture may be 
required and some others may not, in the different ways that were described by the sequence diagrams. The services that have been described here are the ones that are most popular and useful for a system of the features presented here (distributed systems that enhance the regular power grid). Nevertheless, there might be some other ones that are more important for punctual environments or developments.

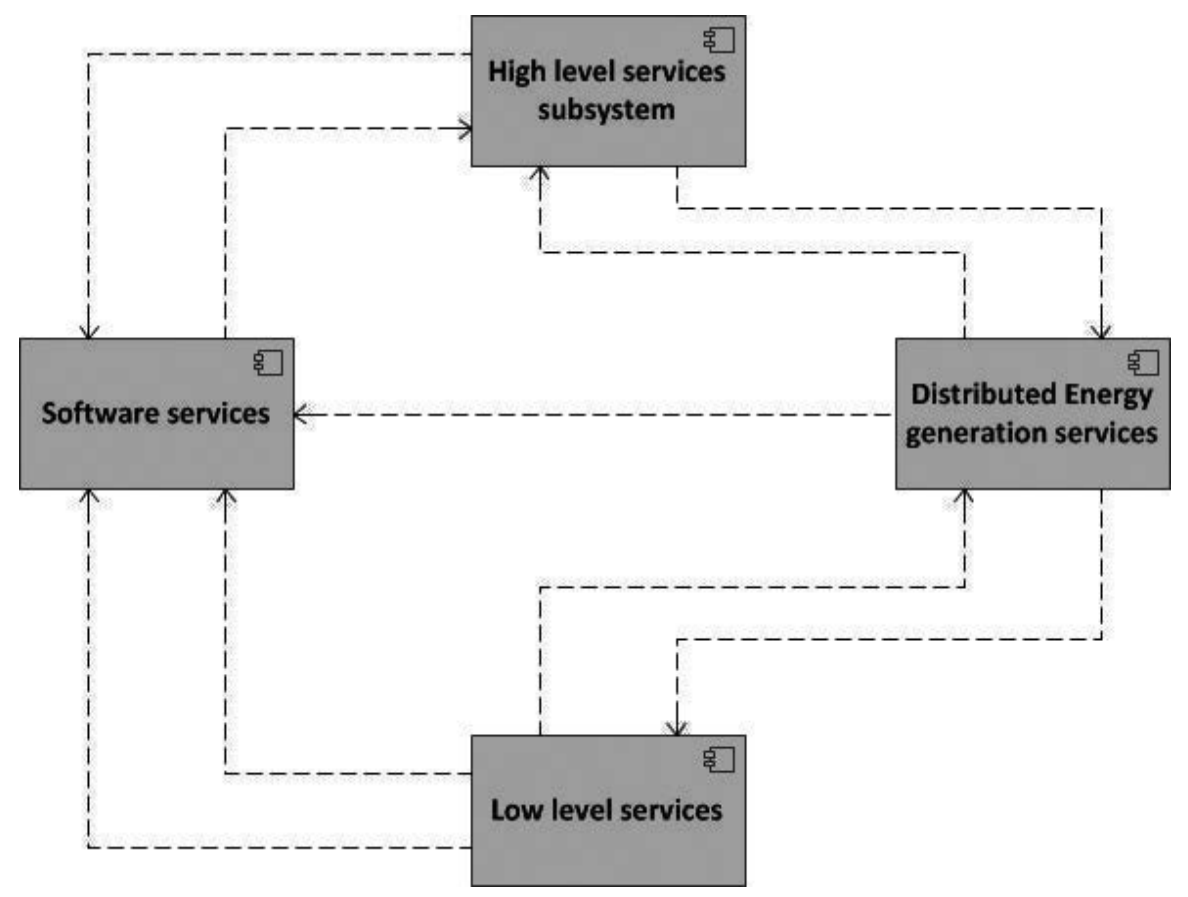

Figure 7. Software subsystems within the generic middleware architecture for the Smart Grid.

\section{A real case study for middleware in the smart grid}

The ideas described in this chapter have been put into practice in several application domains. One of them is regarding a European project called e-GOTHAM [26] that involved building a Smart Grid where several pieces of advanced metering infrastructure and power grid-related appliances were integrated by means of a semantic middleware architecture. The designed semantic middleware architecture was published as a scientific research work [27] and an implementation of it, with all the necessary software components for that specific iteration, was used in the Finnish city of Ylivieska [28]. The overall structure of the power grid network (with the middleware architecture installed in a machine) was also reported as a standardization and dissemination activity [29] and is presented in Figure 8 in a high-level manner.

As described, there are several buildings located in the city of Ylivieska (Concert Hall, schools, a power plant, etc.) using several hardware parts (such as wireless sensor networks) that can 


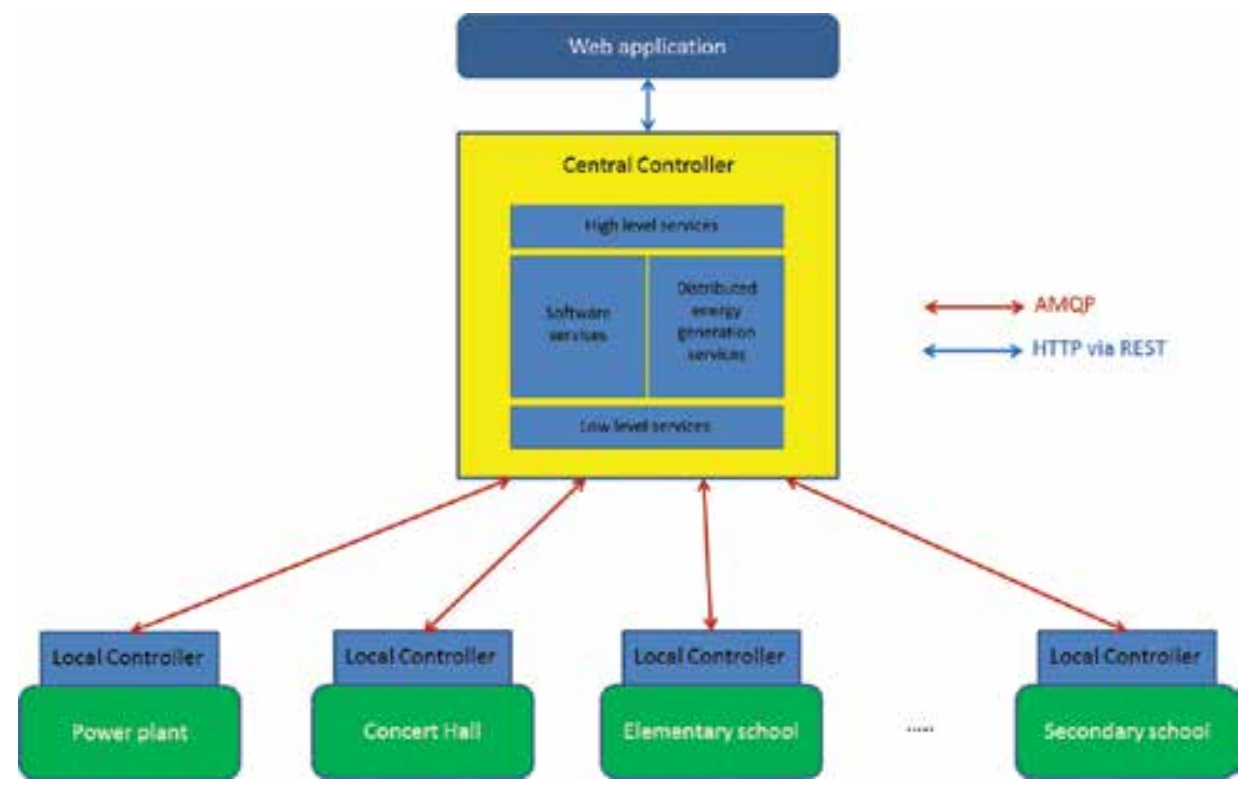

Figure 8. Microgrid deployment with the middleware architecture.

be regarded as AMI. That infrastructure is interconnected by means of local controllers that, depending on their capabilities or whether they are proprietary or open solutions, either can have middleware modules installed, or will rely on the middleware components installed in the central controller. It is this central controller where most of the software modules of the middleware are present, in accordance with the referred architecture (device registration, semantic capabilities, access points to the application layer, etc.). The framework that has been used to have all the software components contained was an open source Enterprise Service Bus, currently known as JBoss Fuse [30]. Collection of data was made from those buildings and presented in a web application with some ancillary functions added (e.g., energy consumption forecast). Other projects, such as I3RES [31], have used similar ideas in order to build middleware architectures for the Smart Grid that have been used in living labs such as Steinkjer, a Norwegian city that often participates in this kind of purposes [32].

\section{Conclusions}

The usage of middleware architectures for the Smart Grid offer clear benefits on the deployments where they are installed: they guarantee that many different pieces of hardware can cooperate seamlessly (regardless of the manufacturer that makes them or the data representation formats that they use), offer different services when applications of devices cannot deliver them, upgrade the system so that it will be able to infer information from the transferred data to be used in the future and improve the services that can be used by the power grid, turning them into a smarter entity capable of providing more information and making smarter decisions. In addition to that there are several middleware modules (hardware abstraction 
modules, context awareness, data aggregation, decision making modules) that are almost mandatory if the full potential of middleware is to be obtained. Fortunately, its widespread usage makes possible that, by collecting information from other proposals or studying the state of the art, can either have some previously developed modules adapted to the need of the middleware architecture in one particular system, or develop new ones with a reduced amount of work, as some guidelines have been offer in this chapter to do so.

The key contribution of this chapter is the summarization of prominent knowledge that has been noticed throughout distributed systems and software architectures research to come to the conclusion that, since there is a plethora of services that will almost always be required, a generic middleware architecture, with a very well defined set of functionalities, can be used whenever middleware is required. This idea can be used for successive deployments that might improve the Smart Grid even further, shortening development times and making a more cost-efficient use of economic resources.

\section{Author details}

Jesús Rodríguez-Molina*

Address all correspondence to: jesus.rodriguezm@upm.es

Research Center on Software Technologies and Multimedia Systems for Sustainability (CITSEM - Centro de Investigación en Tecnologías Software y Sistemas Multimedia para la Sostenibilidad), Technical University of Madrid (UPM), Spain

\section{References}

[1] Alani, M.M., "Guide to OSI and TCP/IP Models". SpringerBriefs in Computer Science. 233 Spring Street New York, New York 10013-1578. USA. ISBN 978-3-319-05152-9. 2014.

[2] Simmins, J.J., "The impact of PAP 8 on the Common Information Model (CIM)," in Power Systems Conference and Exposition (PSCE), Phoenix, Arizona 2011 IEEE/PES, pp. 1-2, 20-23 March 2011. doi: 10.1109/PSCE.2011.5772503.

[3] CEN-CENELEC-ETSI Smart Grid Coordination Group. "Smart Grid Reference Architecture".http://ec.europa.eu/energy/sites/ener/files/documents/

xpert_group1_reference_architecture.pdf. (last accessed November 2012).

[4] Google scholar, https://scholar.google.es/. (last accessed February 2016).

[5] IEEE Xplore, http://ieeexplore.ieee.org/Xplore/home.jsp. (last accessed February 2016).

[6] Elsevier, company history, https://www.elsevier.com/about/company-information/ history. (last accessed February 2016). 
[7] Myerson, J.M., "The Complete Book of Middleware". Auerbach Publications. 6000 Broken Sound Pkwy NW, Florida. USA. ISBN 978-0849312724. 2002.

[8] Serain, D., "Middleware and Enterprise Application Integration". Springer. Schwedenstraße 9, 13359 Berlin, Germany. ISBN 978-1852335700. 2002.

[9] IEEE, "IEEE Transactions on Smart Grid", http://ieeexplore.ieee.org/xpl/RecentIssue.jsp?punumber=5165411. (last accessed February 2016).

[10] Association of Home Appliance Manufacturers, "Smart Grid White Paper: The Home Appliance Industry's Principles \& Requirements for Achieving a Widely Accepted Smart Grid". https://www.aham.org/index.php?ht=a/GetDocumentAction/i/44191. (last accessed December 2009).

[11] Oracle Corporation. "Oracle Fusion Middleware and Microsoft Interoperability. Addressing Enterprise-wide Needs". http://www.oracle.com/technetwork/middleware/fusion-middleware-microsoft-interop-1-131446.pdf. (last accessed February 2006).

[12] Horizon 2020 -European Commission. https:/ec.europa.eu/programmes/horizon2020/. (last accessed February 2016).

[13] Electronic Components and Systems for European Leadership - Joint Undertaking. http://www.ecsel-ju.eu/web/index.php. (last accessed February 2016).

[14] Sensing, monitoring and actuating on the UNderwater world through a federated Research InfraStructure Extending the Future Internet. http://fp7-sunrise.eu/. (last accessed February 2016).

[15] ICT-Based Intelligent Management of Integrated RES for the Smart Grid Optimal Operation. http://www.i3res.eu/v1/. (last accessed February 2016).

[16] GitHub. https://github.com/. (last accessed February 2016).

[17] ResearchGate. https://www.researchgate.net/. (last accessed February 2016).

[18] Object Management Group. http://www.omg.org/spec/UML/2.5/. (last accessed February 2016).

[19] Rodríguez-Molina, J.; Martínez, J-F.; Castillejo, P., and de Diego, R. “SMArc: A Proposal for a Smart, Semantic Middleware Architecture Focused on Smart City Energy Management," International Journal of Distributed Sensor Networks, vol. 2013, Article ID 560418, 17 pages, 2013. doi:10.1155/2013/560418

[20] Bellu, R., “Toutes les Citroën, des origines à nos jours”. Jean-Pierre Delville éditeur, p. 250, Paris, France. ISBN 2-85922-014-3. 1979.

[21] Díaz, V. H.; Martínez, J-F.; Cuerva, A.; Rodríguez-Molina, J.; Rubio, G.; Jara, A. Chapter 9: "Semantic as an Interoperability Enabler in the Internet of Things" from "Internet of Things: Converging Technologies for Smart Environments and Integrated Ecosystems 
(River Publishers' Series in Information Science and Technology)". ISBN 9788792982735. June 2013. Niels Jernes Vej 10, 9220 Aalborg Denmark

[22] Chapter 47: "Java Message Service Concepts" from "The Java EE 6 Tutorial”, 500 Oracle Parkway, Redwood City, California 94065. USA. https://docs.oracle.com/javaee/6/ tutorial/doc/bncdq.html. (last accessed February 2016).

[23] AMQP, Advanced Message Queuing Protocol. https://www.amqp.org/. (last accessed February 2016).

[24] CoAP RFC 7252, Constrained Application Protocol. http://coap.technology/. (last accessed February 2016).

[25] Fielding, R.T. and Taylor, R.N. "Principled design of the modern Web architecture". Proceedings of the 22nd international conference on Software engineering (ICSE '00). ACM, New York, NY, USA, pp. 407-416. DOI=http://dx.doi.org/10.1145/337180.337228. June 2000.

[26] e-GOTHAM ("Sustainable-Smart Grid Open System for the Aggregated Control, Monitoring and Management of Energy"). http://www.artemis-ju.eu/project/index/ view? project=39 Last accessed date: 20th May 2016.

[27] de Diego, R.; Martínez, J.-F.; Rodríguez-Molina, J.; Cuerva, A. “A Semantic Middleware Architecture Focused on Data and Heterogeneity Management within the Smart Grid," Energies, vol. 7, pp. 5953-5994, 2014.

[28] Ylivieska town web site (Finnish). http://www.ylivieska.fi/. Information from tourist guide: http://www.ylivieska.fi/instancedata/prime_product_julkaisu/ylivieska/ embeds/ylivieskawwwstructure/18035_75-Ylivieska-brochure-new_tourist_guide.pdf Last accessed date: 6th April 2016.

[29] e-GOTHAM Validation report for prototype 2 in the three pilots (deliverable D8.4). https://drive.google.com/file/d/0B0xf2wnfLaObV0VrS0Y4aUFGenc/view Last accessed date: 20th May 2016.

[30] Red Hat JBoss Fuse, Download for Development Use. http://www.jboss.org/products/ fuse/download/ Last accessed date: 6th April 2016.

[31] I3RES - ICT based Intelligent management of Integrated RES for the Smart Grid optimaloperation.https://www.citsem.upm.es/index.php/en/projects-en? view=project\&task=show\&id=37 Last accessed date: 6 th April 2016.

[32] Demo Steinkjer Living Lab (Norwegian). https://www.demosteinkjer.no/ Last accessed date: 20th May 2016. 


\section{Section 3}

Energy Storage Systems Technology 

Chapter 7

\title{
Energy Storage Systems for Energy Management of Renewables in Distributed Generation Systems
}

\author{
Amjed Hina Fathima and Kaliannan Palanisamy \\ Additional information is available at the end of the chapter
}

http://dx.doi.org/10.5772/62766

\begin{abstract}
Distributed generation (DG) systems are the key for implementation of micro/smart grids of today, and energy storages are becoming an integral part of such systems. Advancement in technology now ensures power storage and delivery from few seconds to days/ months. But an effective management of the distributed energy resources and its storage systems is essential toensureefficientoperation and long servicelife. This chapter presents the issues faced in integrating renewables in DG and the growing necessity of energy storages. Types of energy storage systems (ESSs) and their applications have also been detailed. A brief literature study on energy management of ESSs in distributed microgrids has also been included. This is followed by a simple case study to illustrate the need and effect of management of ESSs in distributed systems.
\end{abstract}

Keywords: energy storage, distributed generation, energy management, renewable, battery

\section{Introduction}

Distributed generation (DG) and electricity market liberalization have been the key drivers for the evolution of the concept of small-scale energy sources. Growing concerns about climatic changes further encouraged the use of renewable energy sources to ensure energy conservation and sustainability. But integrating renewable energy is turning out to be a real challenge for the smooth operation of DGs. Renewable power especially faces concern regarding power quality. Grid operators face immense issues in scheduling the generated power from the DGs, especially due to renewables and heat-driven energy sources which are difficult to be forecasted. DG may be the key to implement the much talked about micro grids and smart grids of today 
to ensure a clean and green energy portfolio. However, many issues as listed above need to be addressed to enable successful integration of DGs in the power grid. Thus, an effective management of the generating resources in the DG network is very essential.

Energy storages can be incorporated for energy management in many ways. Conventional usage of energy storage devices was mostly for long-term storage applications. But now they can be used for power storage and delivery from few seconds to days and months. Energy storage systems (ESSs) can act as spinning reserves for providing short-term power supply to manage instant variability in DG-generated power. They can compensate for the intermittency and variability of renewable resources and improve the power quality and reliability. ESSs can also provide ancillary services to enable quality power delivery to the end users. Optimized selection, sizing and siting of ESS will be critical for design engineers. Efficient management of energy generated in a DG system can enhance the performance of the system, thereby enabling quality and reliable power delivery. Market prices and other economic dynamics have great impact on the operation of DGs, in which case storage systems can act as added assets to achieve better economic dispatch solutions. Storage systems have proved to improve voltage stability, to smoothen wind power variations, to incorporate peak shaving and load leveling features. The main drawback of storage is its maintenance issues and life cycle failures. Effective implementation and usage of energy storages in the distributed grid requires intelligent and flexible energy management strategies capable of handling the dynamics of distributed systems, while ensuring effective and efficient usage of the storage device. The simplest energy management strategy avoids over charging/discharging of the storage. However, further implementation of hardware-in-loop simulations, optimization algorithms and other intelligent tools and techniques have now been attempted to propose advanced energy management scheme strategies to improve storage lifetime and operability.

\section{Growth of renewable power generation}

Energy crisis emerging from the early 1970s with increased environmental concern was the key factor for setting the foundation for development of renewable sources for electric power generation. Countries like Denmark, Germany and the United States led the green energy mission by creating critical markets and policy targets for development of renewable energy. Awareness on climatic change and its adverse effects further fueled this tremendous drive to reduce emissions and generate environment-friendly energy. Renewable power generation has seen accelerated growth in the developing countries, as it provided a means to reduce dependability on imported nonrenewable fuels. In the earlier days, renewable power was only advocated by scientists and environmentalists but with declining costs and expanding markets it eventually emerged as an implementable solution to improve energy security and diversify the energy portfolio of nations. The overall energy supply from renewables has grown to 76 EJ with a total investment amounting to 214.4 billion USD in 2013 which is a $30 \%$ increase from the scenario in 2004 [1]. Fastest growth of renewables has been recorded in the power sector with 560 GW being generated from renewables in 2013. 
Solar photovoltaics (PV) grabbed the highest growth rate of about 38\% (average growth every year) in these 10 years. Wind power saw rapid expansion all over the world with many countries beginning to join in every year. Though China and the United States saw a mild decline in the wind market, it is still expanding due to technological improvements and fall in prices. Hydropower also increased in the last decade with many joint-venture large-scale projects being implemented. At the same time with microturbines installed on small streams and waterfalls, micro hydropower is also viewed as being a good complementary source to other renewables like wind and solar. Ocean energy is still in developmental stage, with France being the only country to have a functional tidal power plant with rated capacity of $240 \mathrm{MW}$.

\section{Microgrids and DG}

Growing power demand is predicted to be highly devastating for our environment as power generation is the highest contributor to greenhouse gas emissions. Also, rapid depletion of conventional energy resources and increasing fuel prices are crippling the economy of many countries. With technological advances, many renewables are now competing as alternative energy sources to conventional fossil fuels. Conventional power generation was highly centralized due to geographical concentration of energy sources. It also faced many issues like need for extraction infrastructure of generated power, losses in transmission and distribution and lacked the flexibility of being set up at desired locations. This led to conceptualization of DG, wherein the power generation takes place at/near the load centers by many small gridconnected power generating sources called distributed energy resources (DERs) which are mostly renewable in nature. Due to abundance of availability of natural renewable sources like solar and wind, these DERs could be set up anywhere making the DGs flexible, decentralized and modular. They are capable of capturing renewable power and minimizing losses occurring in transmission. This concept has gained a lot of interest due to many reasons as defined by the International Energy Agency [2]. They are congestion on centralized transmission lines, growth of renewables, increased customer demand, electricity market liberalization and environmental awareness. The benefits of DGs are listed below:

1. Flexibility: DGs are flexible in planning, installation, operation and modularity. They can also be started and stopped much more easily as opposed to conventional plants which need startup and shutdown time and costs. Hence they can be easily modulated as per market norms.

2. Reliability: In electrical power systems, it simply means uninterruptible supply to the consumers. This needs high maintenance of transmission network with increased costs for the utility grid. Industrial consumers demand uninterrupted power and hence are more willing for investing in backup and/or local generators. Fuel cells and microturbines are vastly viewed as being excellent small-scale generators for improving system reliability.

3. Power quality: In many developing countries, grid power is still marred with number of power quality issues like voltage sags and frequency deviations. These problems need to 
be addressed to make the systems reliable and improved. DGs can easily be brought into play to improve the power quality and deliver reliable power to the consumer.

4. Green power: As most DERs can be renewables, DGs can be setup to promote green energy and reduce GHG emissions. Emissions omitted or reduced are now being viewed as amounting to energy saved. Many nations have now drafted environmental policies which encourage installation of DGs and urge adoption of green energy.

5. Reducing grid congestion: In order to provide power to remote areas which are located far from generation facilities leads to heavy congestion of transmission lines. Hence setting up of DGs in vicinity of such areas prevents burdening of the grid and avoids investment costs for setting up of new lines.

6. Additional benefits: DG also serve some additional purposes like deferral of upgrades from $\mathrm{T} \& \mathrm{D}$, loss reduction in transmission lines, network support and ancillary services.

Thus DG can benefit power system delivery and mobilize new markets. They can be completely decentralized, serving a localized consumer independent of the grid or operate with the grid to address a part of the local load. Thus any DG which exhibits controllability on its connectivity interface can act as a microgrid. The control of microgrid poses many difficulties and needs extensive strategies to command operability based on grid conditions and customer requirements. They also need unique protection strategies to address any issues arising internally to not affect the power grid. Hence, a DG can be implemented with any combination

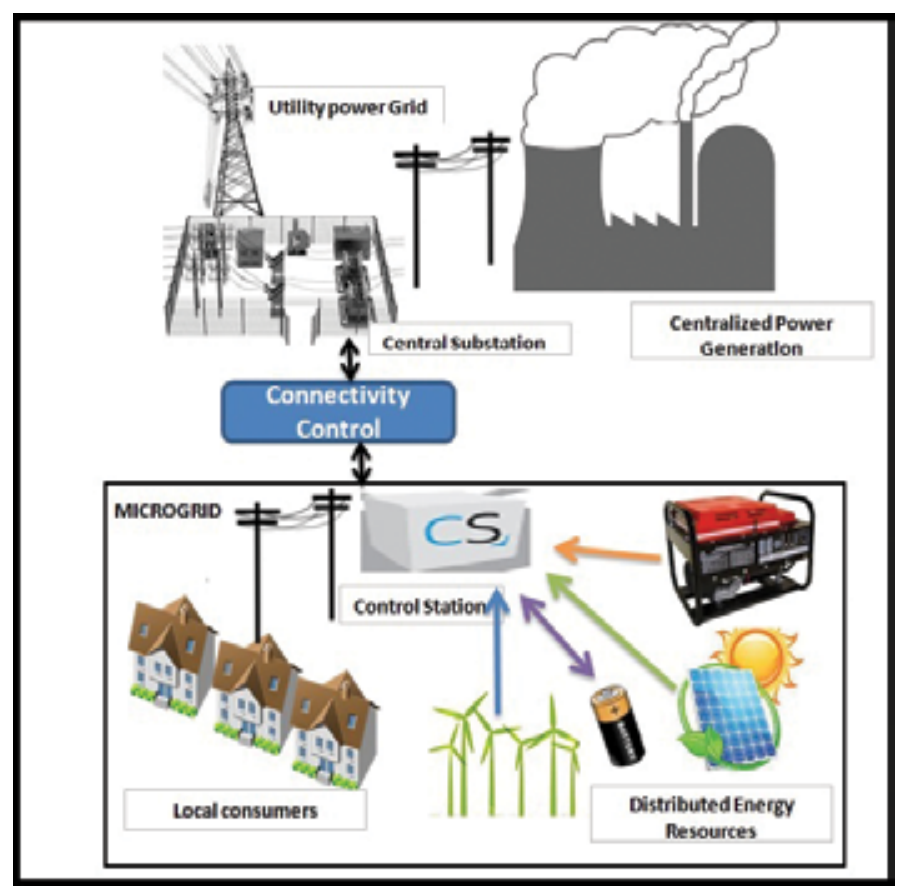

Figure 1. Distributed generation vs conventional centralized generation. 
of generating sources renewable or conventional with/without storage as shown in Figure 1. Thus, many nonrenewable small-scale generators like fuel-cell, diesel-based generators and microturbines are also incorporated into DGs to meet consumer demand [3].

\subsection{Issues and options for integrating renewables in DGs}

Integration of renewable power sources poses many challenges during operation and scheduling of DGs. Intermittency, power quality and price of electricity generated are some of the issues which are being currently addressed in the distributed power scenario. DGs facilitate the power system by providing voltage support and power factor correction applications. However, increased penetration of renewables and extensive decentralization of power sources may cause problems in its voltage profile, making it unstable and unreliable. The major issues which need to be addressed for enabling increased implementation of DGs are explained in detail below:

i. Costs and investments: DGs require installation of different power generating technologies at the load centre, each of which is characterized by a different energy cost. Energy costs include costs for investing in installation, operation, maintenance and replacement costs and are generally coined as cost per kilowatt hour of energy generated. Most of the DERs especially the renewable systems are very expensive compared to existing fossil fuel-based generating systems. As per status reports by REN21 [1], solar PV costs have declined by $50 \%$ in the past 4 years due to widespread awareness and learning towards conceptualization of grid parity. Now, introduction of concepts like microturbines and biomass generation is proving to be cheaper. Also costs of investments can be reduced during planning a DG by selecting the optimal combination of DERs so as to yield maximum energy at lowest costs and adapting optimal operating conditions.

ii. Unpredictability ofrenewable energy system (RES): A dispatchable power is defined as a power source whose output can be regulated to match the demand so as to ensure power balance. Renewables depend on nature and are considered to be nondispatchable due to their continued unavailability and intermittency. Solar power is available only as long as the sun shines and wind is ever varying in both speed and direction. The planning committee must ensure that any intermittency or variability caused in the generation side by these DERs is balanced before being fed to the consumers (or the power grid) to avoid any damage to consumer appliances and to ensure quality. Usually, any party generating power using DGs signs a contract with its local power system operator called the access responsible party (ARP) to ensure stability and operability of the grid as per the grid codes. Grid code encompasses the technical specifications and rules to be abided by the transmitting party to ensure grid safety and security. Any discrepancy henceforth is penalized. Forecasting studies have come a long way in order to enable better planning of renewable power scheduling especially in the wind power sector. Every plant owner, grid operator and energy trader demand high accuracy in forecasting techniques to ease management of DGs and to improve system reliability. 
iii. Power quality issues: Frequency variations and voltage drops and deviations may impact on the quality of power delivered to the consumer. The microgenerators in DG systems lack speed governors and spinning reserves and hence may not be able to provide frequency regulation. In islanded mode of operation the DG becomes more sensitive to variations in voltage profile caused by any variations in load. Additional variations fed in from renewables will further impact the frequency and voltage profile of the system. The main power quality problems faced by DG systems are harmonic distortions and voltage deviations introduced by the many inverters installed to integrate the various DERs like PV panels. Thus, connection of large number of DERs into the DG system requires meticulous planning and control to establish load-frequency and power quality.

iv. Connectivity issues: As explained before, DGs are capable of operating with and without the grid, ensuring reliable power delivery to the consumer. However, the decision to stay connected or operate in isolation requires extensive sensing equipments and intelligent control systems. In the case of voltage faults or grid failure, the microgrid needs to immediately get into islanding the system from the faulty portion of the grid. Similarly, they also must engage in smooth transition from islanded to grid-connected mode after fault clearance. Switching between connected and standalone modes needs to be highly synchronized and safe. Any bidirectional flow of current due to imbalances of voltages at higher renewable penetration needs to be controlled and managed $[4,5]$.

v. Market regulation: Most countries have a monopolistic electricity market where the utility is the sole regulator of electricity distribution to consumers. In such cases, the power generated by the DGs is purchased by the utility under authoritative drafted agreements. Hence, for further penetration of DGs and microgrids a more liberalized market is needed where the DG power could be directly sold to consumers. Some countries only implement marginal norms for purchase and sales of green power which is not sufficient to justify the investment costs of newer technologies. However, liberalization alone is not the solution for this. It may also lead to increased complexity and prices which adversely affect small DGs and renewable generators in meeting scheduled dispatches and buying of backup power.

vi. Regulatory frameworks: Microgrid and DG operators face most problems due to lack of appropriate regulatory frameworks to support and govern investments and operations both in isolated and grid connected scenarios. A fitting legal design for microgrids with market liberalization is very much essential. Proper framework for costing and economic analysis needs to be structured which should recognize value of reliable and nonintermittent power supply.

DGs and microgrids are and will play a pivotal role in meeting future energy challenges. However, the issues discussed above need to be addressed by DG operators, planners and policy makers. Combining energy storage in the portfolio of DERs of a DG will effectively address many of these issues and enable the DG system to operate reliably and securely. 


\subsection{Need for ESS}

ESSs can aid in improving the operation and power delivery of the DG and can help in elimination of uncertainties in the system. Conventional power systems only depended on rotational generators for spinning reserves and ancillary services. But most micro sources in a DG, especially renewable generation units lack this facility and hence depend on external storage to fulfil these requirements. Some of the needs of ESS in DG systems are listed below:

i. Spinning reserve and short-term backup: Fuel-powered plants usually are held at stand by to provide for the spinning reserve and yet, they need considerable time (in minutes) to respond. In the absence of spinning reserve in the case of renewable systems, the ESS can aid in ramping up of power delivery in times of need. Recent advanced storage systems are capable of ramping up in terms of seconds to few minutes. Thus, an effectively managed ESS can replace a much larger spinning reserve. They can also store energy for delivering short-term backup power. They are slower to respond but can be brought into commissioning in about an hour.

ii. Load levelling and peak shaving: Usually utilities operate peak resources and generators to deliver the peak demand power. They are usually combustion engines and gasfired plants which are characterized with lower efficiencies and higher emissions. Efforts are being initiated to reduce the peak of the demand curve by improving the end-user energy efficiency, educating on demand response measures and implementing peak pricing strategies. Energy storage is an attractive option for managing the peak of the demand curve. They are capable of storing energy at low peak times and then discharge it at peak time. Thus it acts as a very responsive and flexible peak reserve. Storage is essential for demand response programs too which will enable a consented and co-ordinated direct control on end users' demand. ESS employed for peak shaving will result in reduced emissions also.

iii. Integration of renewable sources in DG: Wind power is generated mostly at night when the demand is low. Hence, storing this energy and delivering it at demand times enhance the efficiency of the DG system. If the same storage systems are provided at the end-user side, then all the excess wind energy can be transmitted at night time (time of low congestion) and stored near to delivery point. This will also reduce the congestion of T\&D lines at peak times, causing lesser faults and outages. Similarly, solar power is available only as long as the sun shines and hence can be stored at times when generation exceeds demand and discharged at evening peak hours. Increased penetration of solar power resulted in a critical situation called the duck curve in California. This created a huge difficulty for the system providers to ramp up other generators to meet the sudden shift in demand. ESS can help in such scenarios to level the demand curve and aid in ramping up of supply at peak times. They can also help in improving the penetration of renewable generators by eliminating their variability and intermittency.

iv. Power quality support: Integration of renewables poses many power quality issues ranging from flickers to fluctuations and spikes, swells and sags. Then storage 
systems capable of responding rapidly to system fluctuations like flywheels and ultra capacitors can be implemented to maintain power quality standards as per grid codes. Eliminating harmonics, LVRT and transient response can also be managed with an ESS. Flywheels, super capacitors and fast-responding batteries are extensively applied for frequency and voltage control and power quality improvement in DGs.

v. Ancillary services: Ancillary services are those which are needed by the grid operators to sustain stable and reliable operation of the grid. Frequency regulation is an important aspect of ancillary services including load following and energy arbitrage. Future deregulation of markets and introduction of time-based tariffs will create a platform for energy arbitrage. It involves charging of storage with cheap energy at off-peak times and delivering at a higher price at peak times. It goes hand in hand with peak shaving concept but focuses on commercializing the saved energy for maximum profit. However, it is important to note that storage systems used for regulation and load following purposes need to be highly responsive and efficient, else the losses occurring in ramping up/down the storage will outweigh the advantages. Usually conventional generators used for ancillary services are operated at below the rated capacities and hence have lower efficiencies and high emissions. ESS can hence be the emission-free cheaper option for ancillary services thus freeing generators to operate at maximum efficiencies.

\section{Types of ESSs}

Storage and conservation of energy has been practiced by mankind for many decades. Hydro storage and electrochemical batteries have been the traditional face of electricity storage. Based on the technology used, the different ESSs can be classified as shown in Figure 2. Figure 3 shows the share of different energy storage technologies worldwide based on installed capacity.

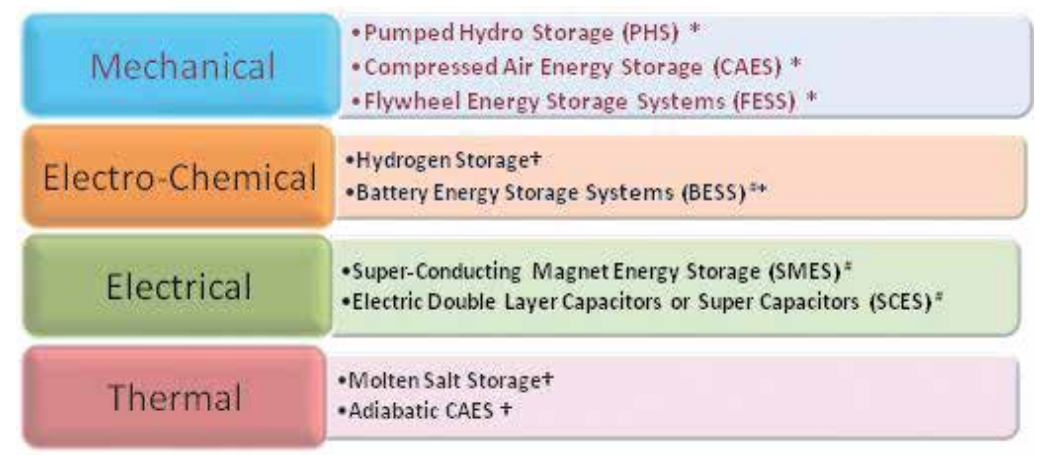

Figure 2. Energy storage technologies. ${ }^{*}$, Deployed and operational; \#, under demonstration; and + , early stage. 


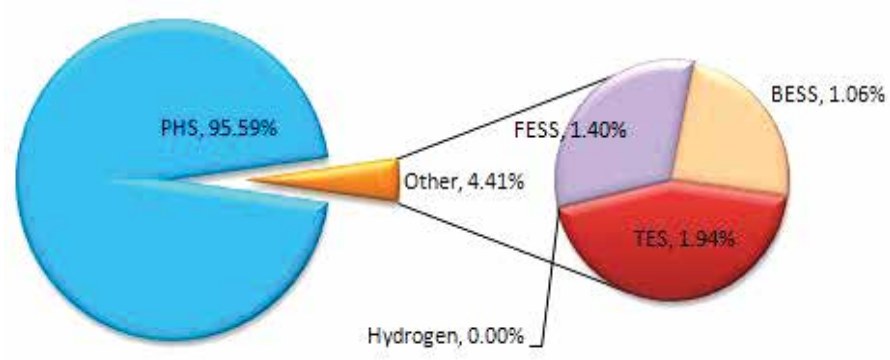

Figure 3. Worldwide operational energy storage technology share based on installed capacity. (Source: DOE database $2015[6])$.

\subsection{Pumped hydro storage (PHS)}

They are commercially installed and most widely operated grid-scale form of storage. PHS employs two reservoirs situated at different heights and pumps water from lower reservoir to upper reservoir using off-peak cheap electricity from the grid. When required the stored water is released to the lower reservoir and electricity is generated through rotating turbines. They are capable of storing huge capacities of energy for many months and have long life times of about $50-60$ years. Their efficiency lies in the range of $70-80 \%$ depending on plant capacity, height difference and type of turbine used. They need extensive investments and long gestation and planning periods. New developments in pumped hydro include features like speed pumping which further improves the system response times for ramping applications.

\subsection{Compressed air energy storage (CAES)}

Compressed air is stored as a form of potential energy in large underground caverns and mines. When needed they are mixed with natural gas and burnt and passed through expansion turbines to generate electricity. This type of CAES has usually low efficiency value of about $50 \%$ and is called as diabatic CAES. If the heat generated during compression is stored as some form of thermal energy and reused to heat air in the discharging process, it is termed as an adiabatic CAES. Such systems are still being explored and when developed will give a very high efficiency of about $75 \%$. They are being used in many places like North America and Australia. Advantages of CAES include large energy capacities with long duration storage. But they also need huge investments and are site-dependent with low round-trip efficiency.

\subsection{Flywheel energy storage systems (FESSs)}

These store energy in the form of electromechanical kinetic energy in a rotating part like an accelerated rotor or rotating cylinder. The concept is based on the usage of stored mechanical inertia of a rotating object. When charging, the flywheel is accelerated and while discharging the reverse process takes place with the flywheel acting as a brake to extract the stored energy. Advanced FESS has high-speed rotors made of light-weight high-strength carbon materials spinning at twenty to fifty thousand rpm under vacuum. They are capable of supplying high 
power rating at minimum response times and are very suitable for ramping and spinning reserve applications. Other attractive features of a FESS include high efficiency, high power rating and long life with minimum to no maintenance. The major drawback is the high selfdischarge, occurring due to deceleration and resistive losses at bearings.

\subsection{Hydrogen storage}

Electricity is used to split water into hydrogen and oxygen in an electrolyzer, and the hydrogen generated is stored under pressure in separate tanks. On requirement, this hydrogen is passed with oxygen/air into a fuel cell to generate electrons and water (a reverse electrolysis process). Thus, it is truly a form of very clean energy as water and heat are the only byproducts of the entire process. This is also called as a regenerative fuel cell (RFC). They possess high modularity, scalability, energy and power capacities. But they have low-to-medium efficiencies of $50 \%$ and suffer from self-discharge.

\subsection{Battery energy storage systems (BESSs)}

These are the most widely implemented and commercially used storage systems in power system applications. The basic idea is to convert electricity into some electrochemical form and save it as electrolytes inside a cell. While discharging, the electrolytes react with the electrodes in the cell and reverse reaction generates electric current. Over the years, many types of batteries have been developed each having a varied range of characteristics thus making the battery storage technology highly versatile and multipurpose. Nonrechargeable batteries are known as primary batteries and they are mostly used in military and medical applications. A hierarchy of rechargeable/secondary batteries is shown in Figure 4.

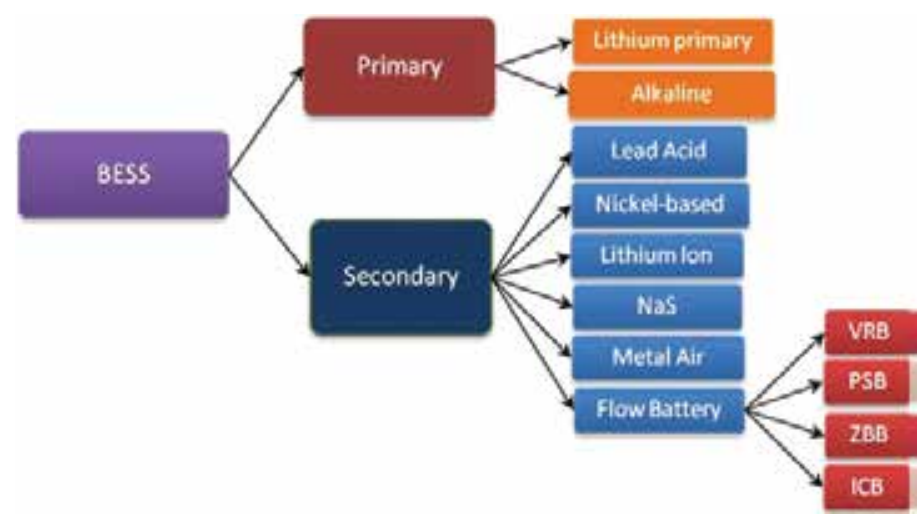

Figure 4. Battery storage systems' hierarchy.

Lead-acid batteries are in usage since the 1890s and recent advancements like valve-regulated batteries, absorbent glass mat batteries are being proposed to improve battery performance and reduce maintenance. Nickel cadmium batteries have been used in applications for stabilizing wind energy but have been discouraged due to environmental hazards from 
cadmium disposal. Lithium-ion batteries have very high energy density and find extensive applications in portable electronic systems. Recent lithium-polymer systems are also being developed with excellent features for renewable integration. $\mathrm{NaS}$ batteries are finding increased applications in renewable systems with high power density and efficiency. Metalair batteries are recently being explored. Lithium-air batteries are most attractive with highspecific energies and zinc-air batteries are also feasible. But metal-air batteries are extremely prone to risk of fire due to high reactivity between metals and air/humidity. Flow batteries are rechargeable batteries which store the liquid electrolytes externally in separate tanks. This helps in increasing their energy capacity by many folds. They are highly modular and scalar as their power capacity can be added by adding up of electrode cells. Instant recharging can be done by simply replacing the electrolytes in the tank. Recyclable electrolytes make the system highly efficient and they are capable of operating for many thousands of cycles. They are also called redox batteries as reduction-oxidation reactions occur in the battery during charging/discharging process. Some types of flow batteries are vanadium redox battery (VRB), polysulphide bromide battery (PSB), zinc-bromide battery (ZBB) and iron-chromium battery (ICB). A detailed exploration of battery storage systems and their characteristics have been presented in [7].

\subsection{Super-conducting magnet energy storage (SMES)}

Each SMES unit has a superconducting coil maintained at low temperature, a power conditioning equipment and a cooling system. Existence of electricity in an electromagnetic form has been discovered ages before when supercooled metals were found to exhibit superconductivity. Employing this feature for storing electric energy began in the late 1960s when strong magnetic fields formed by superconducting coils at extreme low temperature such as 4 Kelvin were explored. Recent research has led to development of materials which can function at 100 Kelvin also. The system has high efficiency but lower energy capacity and power ratings. Also, cooling systems increase the costs and maintenance of the system and it is still in the early stages of demonstration.

\subsection{Electric double layer capacitors or super capacitors}

These store electric current in the form of electrostatic charges. Usually it is made of a parallel plate structure with dielectric between them which stores the charge. This is the technology which bridges the gap between conventional dielectric capacitors and batteries as they can store large capacity of energy, comparable to batteries. Since they do not involve any conversion process (as in batteries) they are very fast and capable of rapid charge and discharge cycles. They are highly efficient $(<90 \%)$, environmentally safe, easily recyclable and suitable for frequency support applications. However, they suffer from very high self-discharge and find increased applications in electric vehicles and traction systems.

\subsection{Thermal energy storage (TES)}

With increased solar power penetration, thermal storage has gained a lot of perspective. Excessive heat energy can be stored for later usage in buildings or in externally stored 
secondary energy carriers. Ice-based and Molten salt technologies are being developed and once under operation are expected to share a huge part of electric demand for heating purposes. Ice-based thermal storage includes latent heat storage and when used with solar power generation is claimed to achieve exemplary efficiencies.

\section{ESSs for energy management of DGs}

Section 3 detailed the various issues faced in integrating renewable systems and the subsequent need for ESSs. It provided a general understanding on the various applications for which ESSs can be used. Some applications like power smoothing and frequency regulation require storage systems capable of charging/discharging high power in short duration. Whereas, arbitrage and peak shaving applications need more energy capacity to withhold the stored energy with low self-discharge. Depending on these applications, appropriate type of storage needs to be selected and sized to be integrated with the DG. As storage systems are quite expensive, they need to be managed effectively to ensure their longevity and performance, else they may cause O\&M issues, reduced performance and premature failure. A proper management of both the storage as well as the generating units is necessary to ensure effective utilization of the DERs. Hence, energy management has been a key topic of interest to renewable energy researchers and developers. Figure 5 depicts the components of an energy management system.

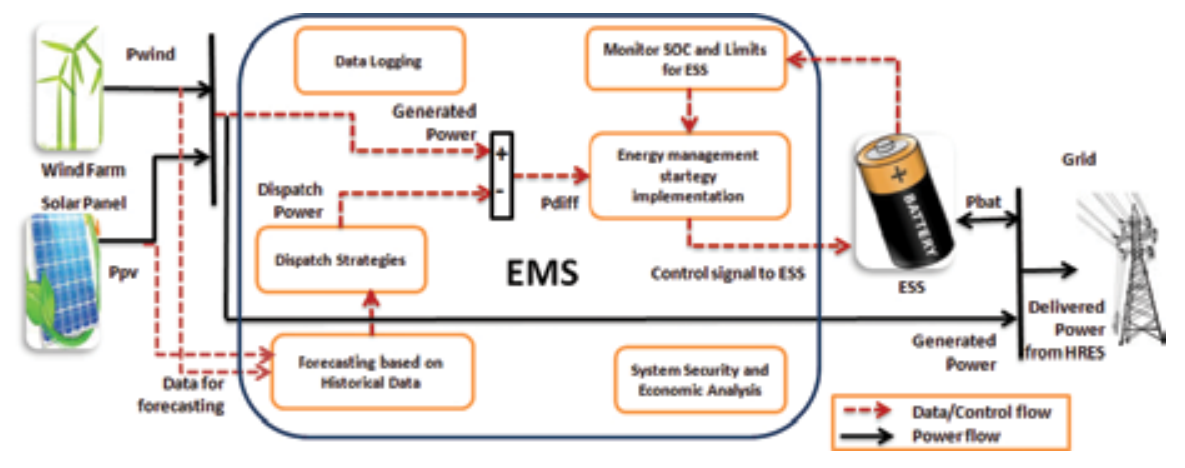

Figure 5. Components of an energy management system.

Energy management strategies are a crucial part of planning of autonomous and standalone power systems incorporating renewable power [8]. A storage system can be operated to address many applications as detailed before in a microgrid, and the management strategy will have to be proposed in view of attaining the predetermined objectives without affecting the system as well as the storage system. As Nykamp et al. [9] stated in their study, from a Distribution System Operator (DSO) perspective a storage system's usage is to be primarily oriented towards peak shaving and renewable integration applications. But, on the contrary, a private energy trader would want to utilize the storage system to maximize profits by arbitrage and ancillary services that would earn money. A vivid comparison of different 
applications of the same storage in a distribution grid has been accounted with simulation results in [9]. Generally, energy management is based on state of charge (SOC) of the energy storage devices so as to avoid any over/under charging issues [10-12]. Reihani et al. [13] simulated grid-scale battery storage to implement peak load shaving, power smoothing and voltage regulation of a distribution system. Tewari and Mohan [14] analyzed and managed a sodium-sulphide $(\mathrm{NaS})$ battery to shift wind power generation from off-peak to peak-load times and explored market participation opportunities for the battery. Díaz-González [15] managed a flywheel-based storage device to achieve wind power smoothing for grid integration using a vector-controlled algorithm and Chandra et al. [16] used a battery system for frequency damping of system oscillations. Zhou et al. [17] investigated the sizing of a standalone PV-hydrogen system based on an optimized energy management strategy aimed at maximizing efficiency of operation of the hydrogen system. Garcia et al. [18, 19] proposed an intelligent energy management system to determine the power sharing between the hydrogen and battery storage based on operating costs in the hybrid standalone system and yielded better results as against the simple state-based energy management strategies. Cheng et al. [20] used PSO with roulette wheel redistribution mechanism to include power balance equality constraints in the energy management strategy. The energy management strategy considers the effects of depth of discharge of the battery storage and enables extending longevity by penalizing charging and discharging based on SOC levels.

Gamarra and Guerrero give a detailed overview of the different optimization techniques applied to microgrid planning and energy management. Wang et al. [21] presented a hierarchical energy management strategy for minimizing operation costs while optimizing the uncertainties in the wind and load and tested it on RT_Lab real-time platform. Mohammadi et al. optimized the management of a microgrid using Hong's estimation [22] and stochasticbased frameworks [23] to minimize costs and manage uncertainties. They also proposed a unit commitment formulation for the microgrid based on cuckoo search algorithm [24]. Chaouachi et al. [25] attempted an online energy management strategy involving intelligent and multiobjective optimization techniques for a hybrid microgrid to minimize costs and emissions. Chen et al. [26] employed intelligent fuzzy-based management of battery SOC in a DC microgrid integrating renewable source resulting in improved battery lifetime. Feroldi et al. [27] conceptualized control based on receding horizon strategy giving highest priority to wind generation and using hydrogen energy as the least priority source. The strategy enabled an improvement of about $88 \%$ in power supply delivery. R. Palma-Behnke et al. [28] employed the rolling horizon strategy to manage a wind-PV-diesel-storage hybrid system by evaluating set points for generating and storage units for optimized operation of the microgrid based on demand side management. Stochastic-based dynamic programming is employed in [29] to adapt to uncertainties of wind energy and market price variations.

ESSs also find applications in distributed systems for managing renewable power curtailment occurring due to transmission system constraints [30]. Fu [31] adapted a distribution feeder and operated it as a microgrid by integrating renewable sources and ESSs to measure and observe power quality issues. Silva-Monroy and Watson [32] addressed some core issues encountered while integrating energy storage devices for market management applications 
for the grid. Abdeltawab et al. [33] also proposed a market-oriented strategy for managing a wind-battery storage system with the aim of earning maximum profit in a deregulated market structure.

\section{Energy management of energy storage for a hybrid renewable system - a case study}

A hybrid renewable system consisting of a wind turbine with a solar panel is considered in the case study to understand the need for storage system and to simulate how management of the energy storage will help in improving the reliability and performance of the power system. The wind turbine produces wind power through an asynchronous induction machine at a rated power of $200 \mathrm{~kW}$ at $400 \mathrm{~V}$. A $75 \mathrm{~kW}$ solar panel is connected to the power system through an inverter to convert the dc current into alternating current to deliver the load. The system is a grid-connected power generating system which delivers the power generated to the grid through a small substation. The hybrid system is simulated by modeling the wind and solar power models as detailed in equations (2) and (2) using Matlab coding software with wind speed and irradiation data fed from real-time measured data from a wind farm site.

a. Wind turbine modeling: The turbine generates power $P_{w}$ depending on the wind speed $v$. If the speed is lesser than the cut-in speed $\left(v_{c i}\right)$ or above the cut-off speed $\left(v_{c o}\right)$, then the turbine does not generate power. For wind speeds greater than $v_{c}$ and rated speed of turbine $v_{r}$ the power is proportional to cubic of wind speed. $C p(\lambda, \beta)$ is the power co-efficient between the tip-speed ratio $\lambda$ and the pitch angle $\beta . \rho$ is the density of air and $A$ denotes the wind turbine swept area.

$$
P w(v)=\left\{\begin{array}{lc}
0, & v \leq v c i \text { or } v \geq v c o \\
0.5 \rho A C p(\lambda, \beta) v^{3}, & v c i \leq v \leq v r \\
P \text { rated wt, } & v r \leq v \leq v c o
\end{array}\right.
$$

$b$. Solar power modeling: Let $Y p v$ be the rating of the solar panel in kilowatt. Solar power generated $P_{p v}$ in a panel is dependent on the solar irradiation $G c$ and temperature $T c$ is incident on the panel. $\alpha$ is the temperature coefficient and fpv is the derating factor of the solar panel. Then, GSTC and TSTC are the irradiation and temperature values at standard test conditions.

$$
P_{p v}=Y_{p v} f_{p v} \frac{G_{c}}{G_{S T C}}\left[1+\alpha\left(T_{c}-T_{S T C}\right)\right]
$$

The simulation is run based on real-time data recorded from a wind test station in southern Tamil Nadu, India. The wind speed profile and the irradiation and temperature are shown in Figures 6 and 7, respectively. Data measurements were conducted for a period of ten days and simulations carried out. 


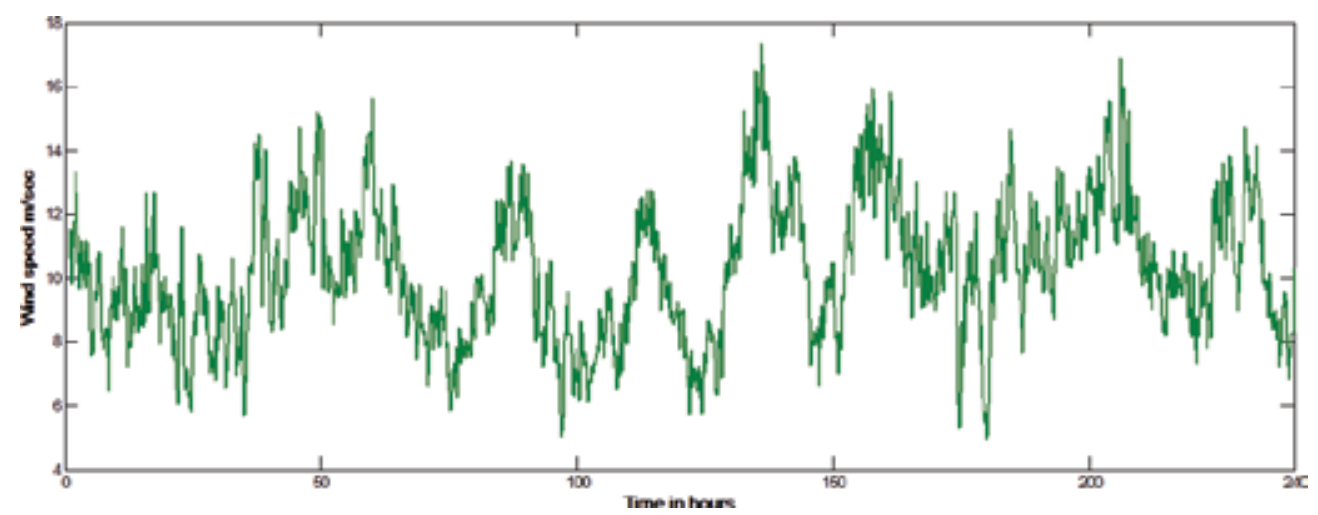

Figure 6. Wind speed in meter per second.

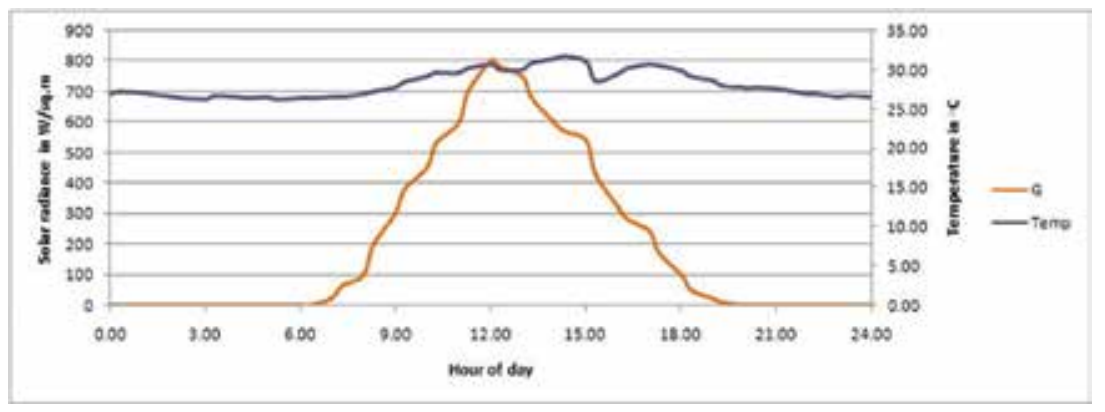

Figure 7. Solar irradiation and temperature.

The sum power thus generated by the wind and the solar system calculated using equations (2) and (2) is considered to be $P_{g e n}$ and is found to be highly intermittent and varying. It becomes a very difficult task for the system operator to schedule this power to the grid. Such high invariabilities also pose to destabilize the grid and tend to affect the quality of the delivered power. Hence, the power generated needs to be dispatched based on a scheduling strategy which eliminates the intermittencies and meets the demand. Also, it is to be noted that maximum wind generation occurs at times like late nights when the load on the grid is at minimum and hence this power cannot be dispatched. Any sudden surges created by wind power cannot be accommodated immediately and the hybrid renewable energy system (HRES) must adhere to feed in power limits of the grid. So, the scheduling strategy takes into account a certain amount of peak shaving and ramp rate-limiting features to address these issues. The power generated (denoted by $P_{g e n}$ ) and the power to be dispatched (denoted by $D e m$ ) is simulated and plotted in Figure 8. There is a need to manage the energy generated to meet the dispatch curve. Let us explore some energy management strategies to improve this power scenario and understand the need and role of energy storage in the form of case studies developed for a wind-PV HRES using Matlab. Each case study clearly presents the management strategy implemented to operate the storage system optimally. 


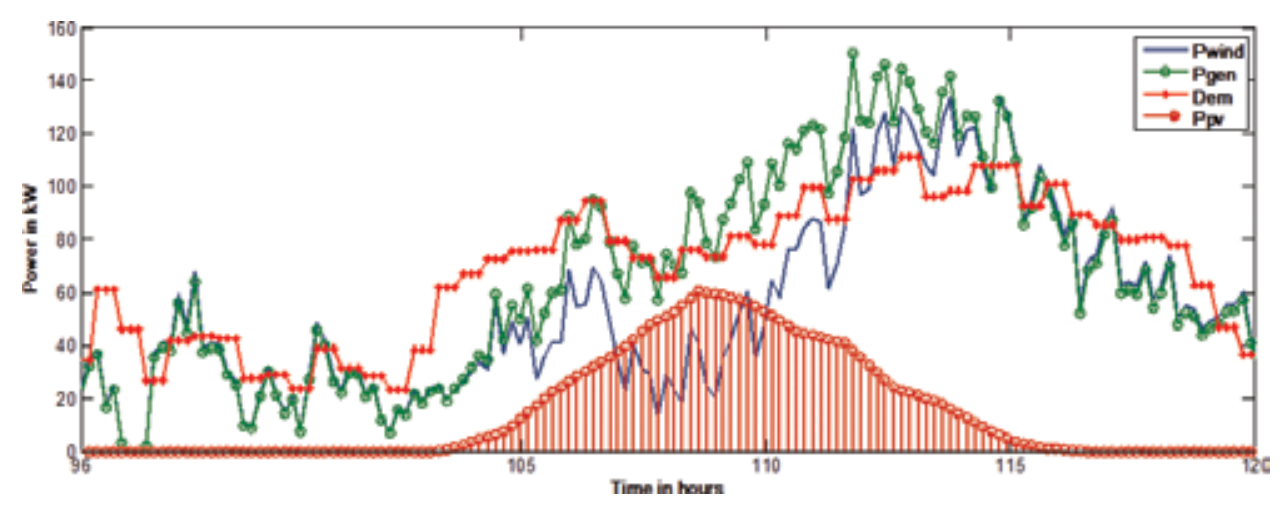

Figure 8. Power graph showing generated and scheduled power.

\subsection{Case 1: Without storage}

In this scenario the power generated by the HRES has to be delivered as it is generated. So at times when $P_{\text {gen }}>$ Dem the demand is met and the excess generation is stalled leading to spilling losses. At times when $P_{\text {gen }}<D e m$, the generated power is entirely fed to meet the demand and the excess demand has to be shed thus leading to shedding losses. Additionally, the shed power has to be met by some other form of conventional energy like a backup diesel generator. The power curve showing power mismatch between the $P_{\text {gen }}$ and Dem is plotted as shown in Figure 9.

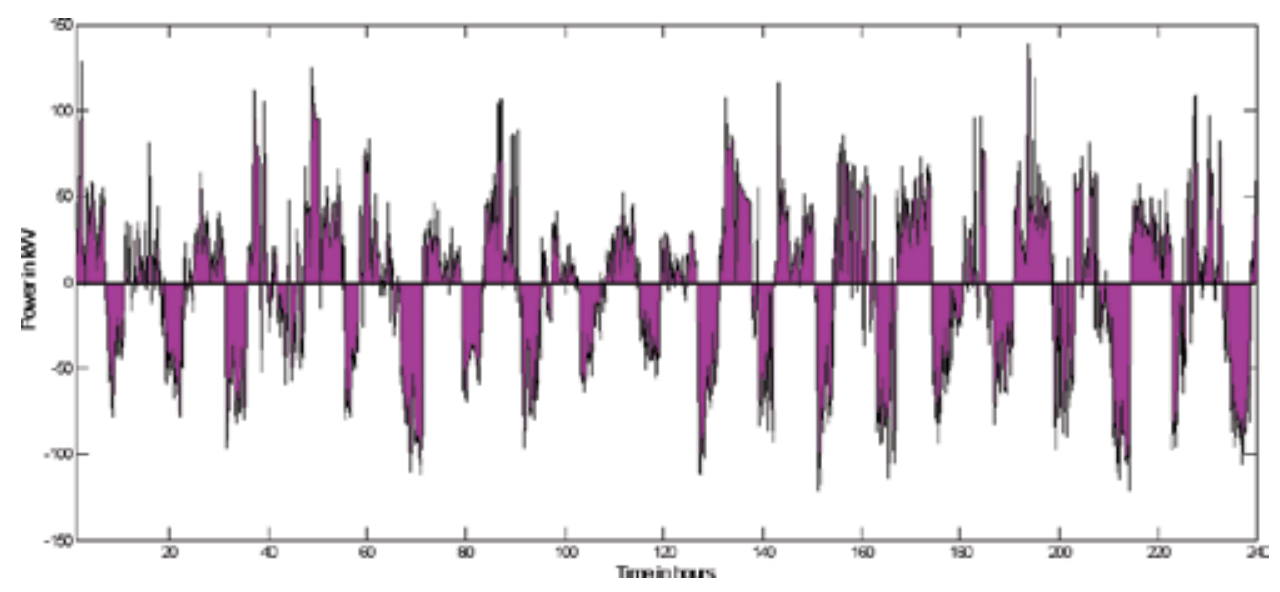

Figure 9. Power mismatch curve.

Evaluation of simulation results show that the total energy delivered by the HRES for the simulated duration of 10 days is $18.64 \mathrm{MWh}$, with a total of 4.5 and $4.8 \mathrm{MWh}$ of energy lost due to spilling and shedding, respectively. This led to an overall estimated loss of power supply probability (LPSP) of $26.14 \%$ [34]. 


\subsection{Case 2: With a single battery bank}

In this scenario, consider a VRB with the system to improve the power delivery and reduction of losses. Flow batteries have been proving to be an excellent solution for integration of renewable systems due to their ability to store huge capacities for longer time and deliver over 10,000 charge/discharge cycles. A detailed sizing study was conducted to evaluate the optimum size of the battery using Bat optimization algorithm. The methodology can be referred in [34]. The optimum size thus found for this case is $1250 \mathrm{Ah}$ operating at $120 \mathrm{~V}$. The specifications of the VRB battery are tabulated in Table 1. VRB battery is a modular structure, with each module has a rating of $625 \mathrm{Ah} 48 \mathrm{~V}(30 \mathrm{kWh})$. Hence the VRB battery has two parallel banks, each having three modules in series. Thus, the total number of battery modules is 6 . The VRB battery is modelled based on SOC given by

$$
\operatorname{SOC}(t)=\operatorname{SOC}(t-1)+P_{b}(t) \times \frac{\Delta t}{E_{\text {bess }}}
$$

Ebessis the energy capacity of the battery in kilowatt hour and $\mathrm{Pb}(t)$ is the power to be charged/ discharged by the battery in time duration $\Delta t$. A simple energy management strategy is developed to avoid any over/under charging of the battery. It is shown in Figure 10. The power delivered $\left(P_{d i s}\right)$ curve plotted against Dispatch $(D e m)$ and the power mismatch $\left(D e m-P_{d i s}\right)$ curves are shown in Figure 11. Figure 12 shows the SOC of the VRB battery.

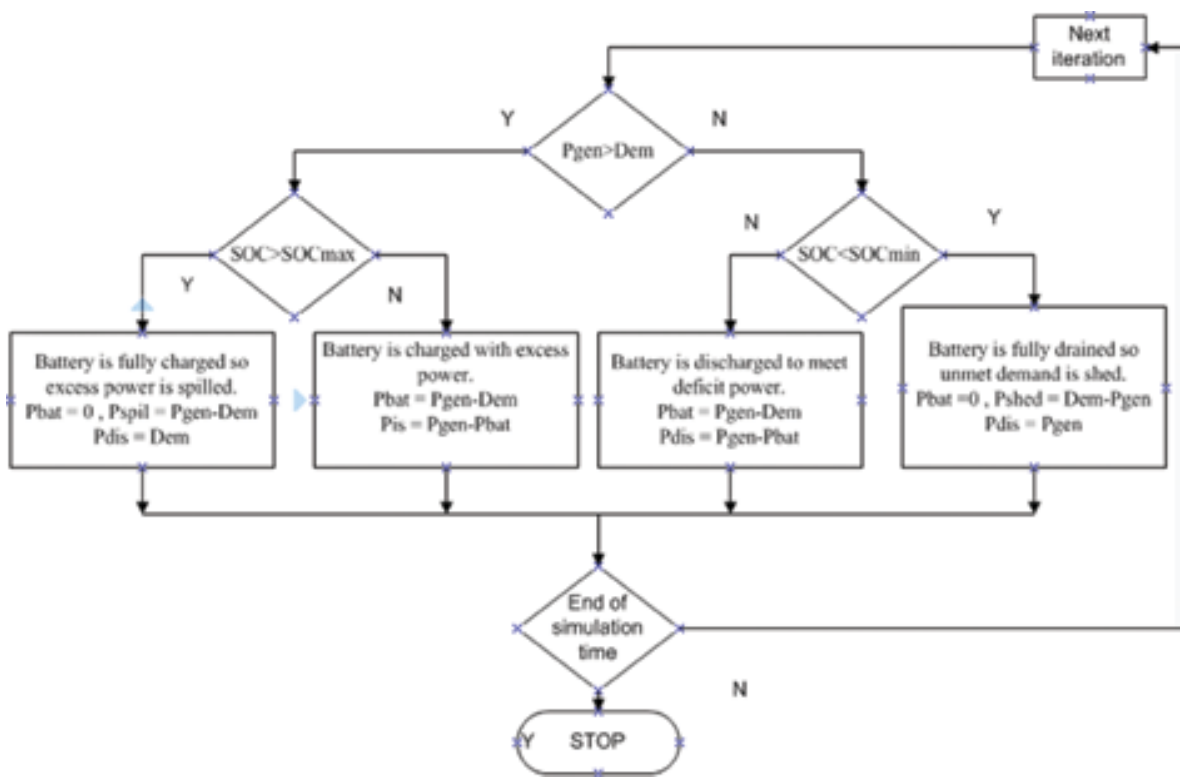

Figure 10. Energy management of VRB battery. 


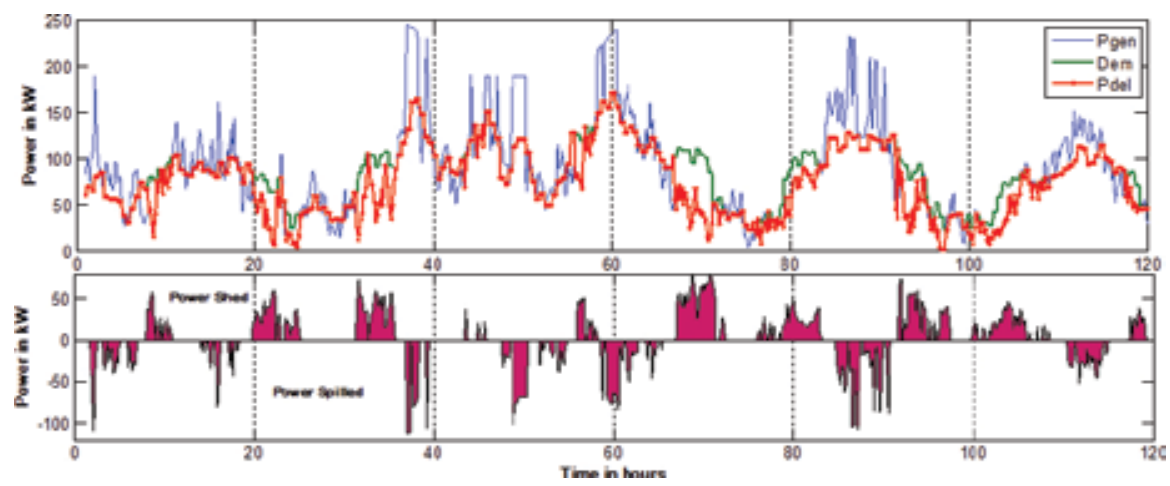

Figure 11. Power delivered curve (Dem vs $\left.P_{d i s}\right)$ and power mismatch curve.

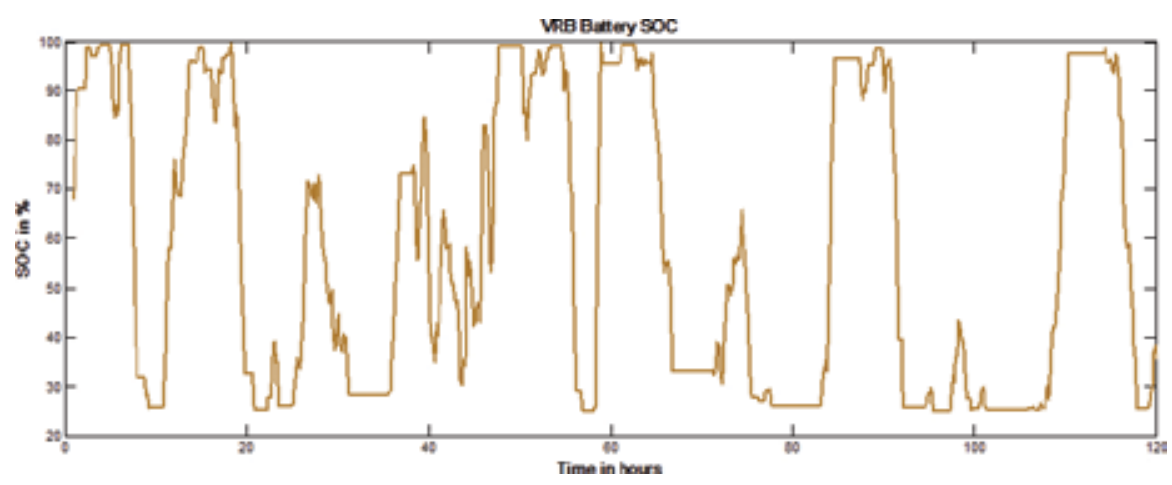

Figure 12. SOC of VRB battery.

The total energy delivered by the HRES is now increased to $23.2 \mathrm{MWh}$, and the shedding and spilling losses are reduced to 2.6 MWh and 2.2 MWh, respectively, resulting in a profit of 1020.4 $\$$ in the simulation period for 10 days. Assuming VRB battery energy cost of about $600 \$ / \mathrm{kWh}$, the payback period is evaluated as about 6 years. The LPSP is also reduced to $11.25 \%$.

\subsection{Case 3: With hybrid battery energy storage}

In this scenario, let us consider a VRB and a lithium-ion battery system to be integrated in parallel with the system to improve the power delivery and reduction of losses. Lithium-ion batteries are characteristics of high power capacities which can be utilized to balance the intermittencies experienced in this case. The lithium-ion battery is operated for high power requirements and VRB for storing energy for longer duration. Hence, Li battery is of higher power capacity and VRB is sized to be with higher energy capacity. Power limits for Li and VRB batteries is set to be 60 and $30 \mathrm{~kW}$, respectively. The specifications of the Li-ion battery are shown in Table 1 . The VRB battery has an energy capacity of $625 \mathrm{Ah}(75 \mathrm{kWh})$ and is formed by connecting three $625 \mathrm{Ah} 48 \mathrm{~V}$ modules in series. The Li-ion battery capacity is $250 \mathrm{Ah}$ (30 
$\mathrm{kWh}$ ). A simple energy management strategy is developed to avoid any over/under charging of both the batteries as shown in Figure 13. As shown, the VRB battery is checked for power limits and operated. Li-ion battery is operated only when the power requirement is beyond the limit of VRB battery or when the VRB battery is completely full/dry. The power delivered $\left(P_{d i s}\right)$ curve plotted against Dispatch $(D e m)$ and the power mismatch $\left(D e m-P_{\text {dis }}\right)$ curves are shown in Figure 14. Figure 15 shows the SOC of the VRB and Li-ion batteries. It is to be noted that the Li-ion battery undergoes more charging/discharging cycles due to its smaller capacity and greater depth of discharge.

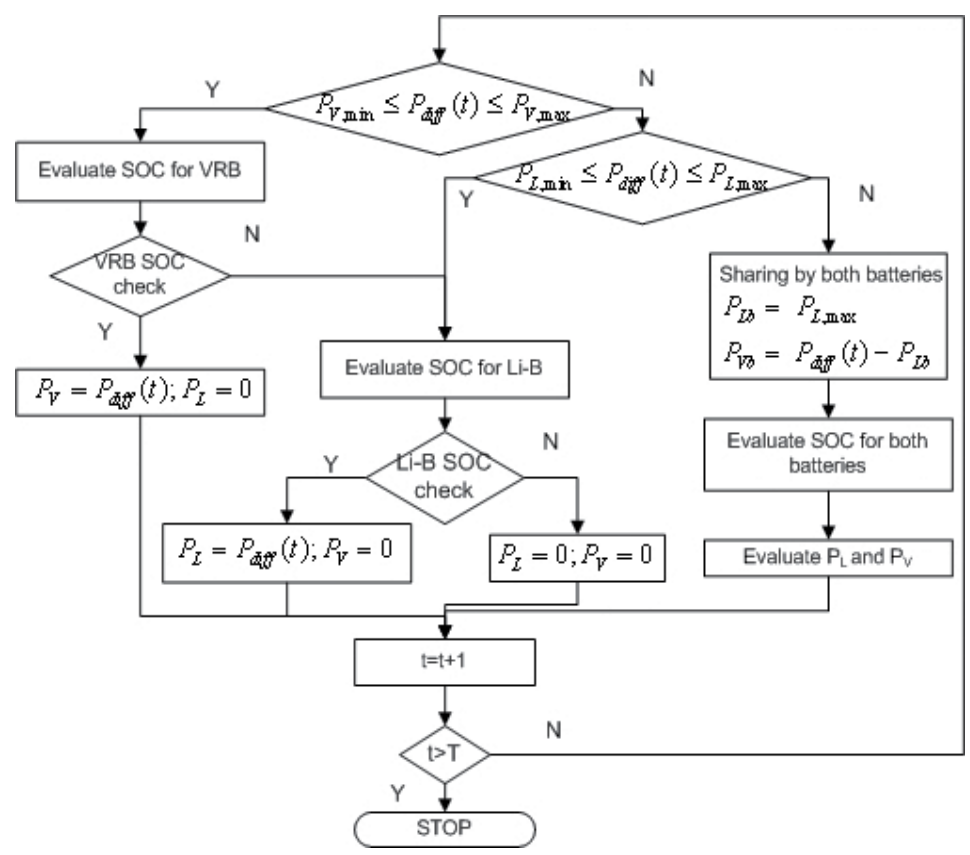

Figure 13. Energy management of hybrid BESS.

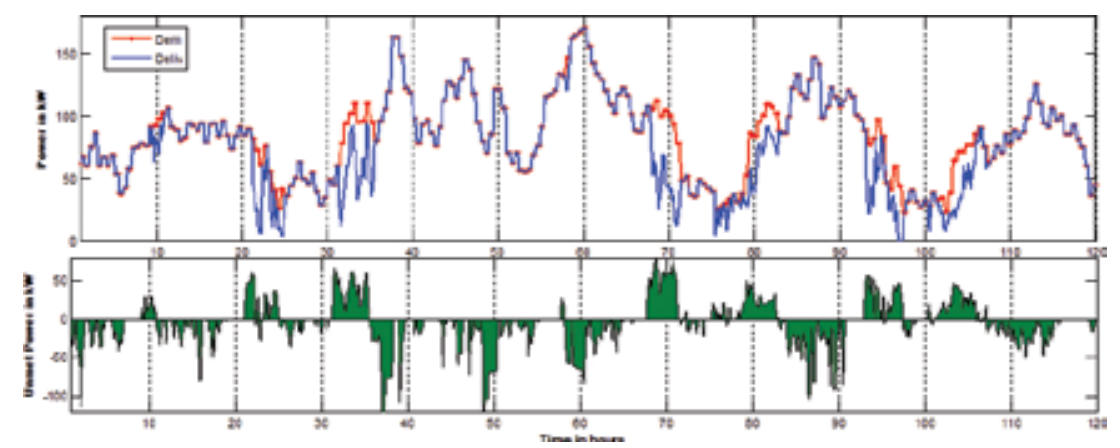

Figure 14. Power delivered curve (Dem vs $\left.P_{d i s}\right)$ and power mismatch curve. 

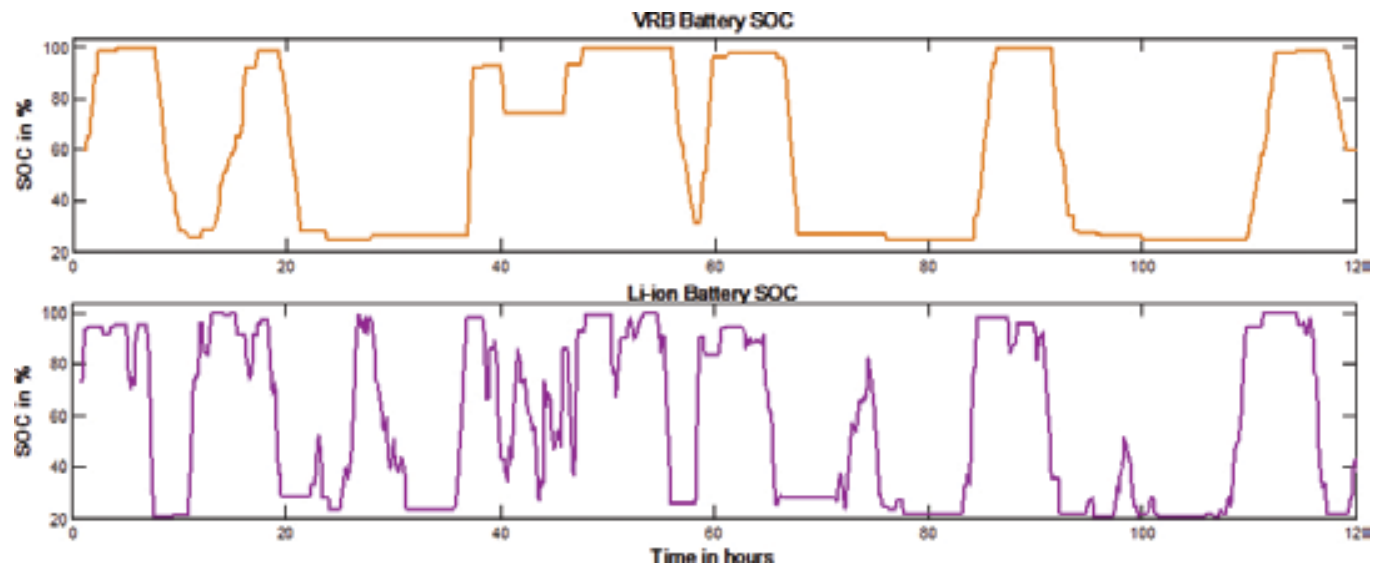

Figure 15. SOC of VRB and Li-ion battery.

The total energy delivered by the HRES is now increased to $23.2 \mathrm{MWh}$, and the shedding and spilling losses are reduced to 1.9 and $1.7 \mathrm{MWh}$, respectively, resulting in a profit of $19,697 \$$ in the simulation period for 10 days. Assuming the VRB battery energy cost of about $600 \$ / \mathrm{kWh}$ and the Li-ion battery energy cost of about $500 \$ / \mathrm{kWh}$, the payback period is evaluated as about 4 years. The LPSP is also reduced to $8.28 \%$.

\subsection{Case 4: With dual VRB battery energy storage}

: In this scenario, let us consider a dual VRB to be integrated in parallel with the system to improve the power delivery and reduction of losses. VRB battery modules of capacity which is the same as the ones used in Case 2 is taken but instead of operating them as a single 150 kWh system, they are now operated as two separate VRB battery banks. Each bank has three $625 \mathrm{Ah} 48 \mathrm{~V}$ batteries in series. Thus let VRB1 and VRB2 be two separate VRB batteries, each of size $90 \mathrm{kWh}$. Cost of investment for battery remains the same, as the number of battery modules used is the same. But more power electronic components are used as both batteries need individual control. They are operated in master-slave relationship to enable complete charge/discharge cycles to improve lifetime and performance of the VRB. First VRB1 is set as master battery which undertakes all charging and VRB2 is the slave battery which only takes care of discharging cycles. Once either battery reaches its peak limits (fully charged or discharged), the roles of batteries are interchanged. In cases when the power requirement exceed the power limit of any one battery then both batteries also operate in parallel to deliver the required power mismatch. A simple energy management strategy is developed as shown in Figure 16 to avoid any over/under charging of both the batteries implemented using fuzzy logic for optimal power sharing between the dual batteries. The power delivered $\left(P_{d i s}\right)$ curve plotted against dispatch $(D e m)$ and the power mismatch $\left(D e m-P_{d i s}\right)$ curves are shown in

Figure 17. Figure 18 shows the SOC of the two VRB batteries, respectively. 


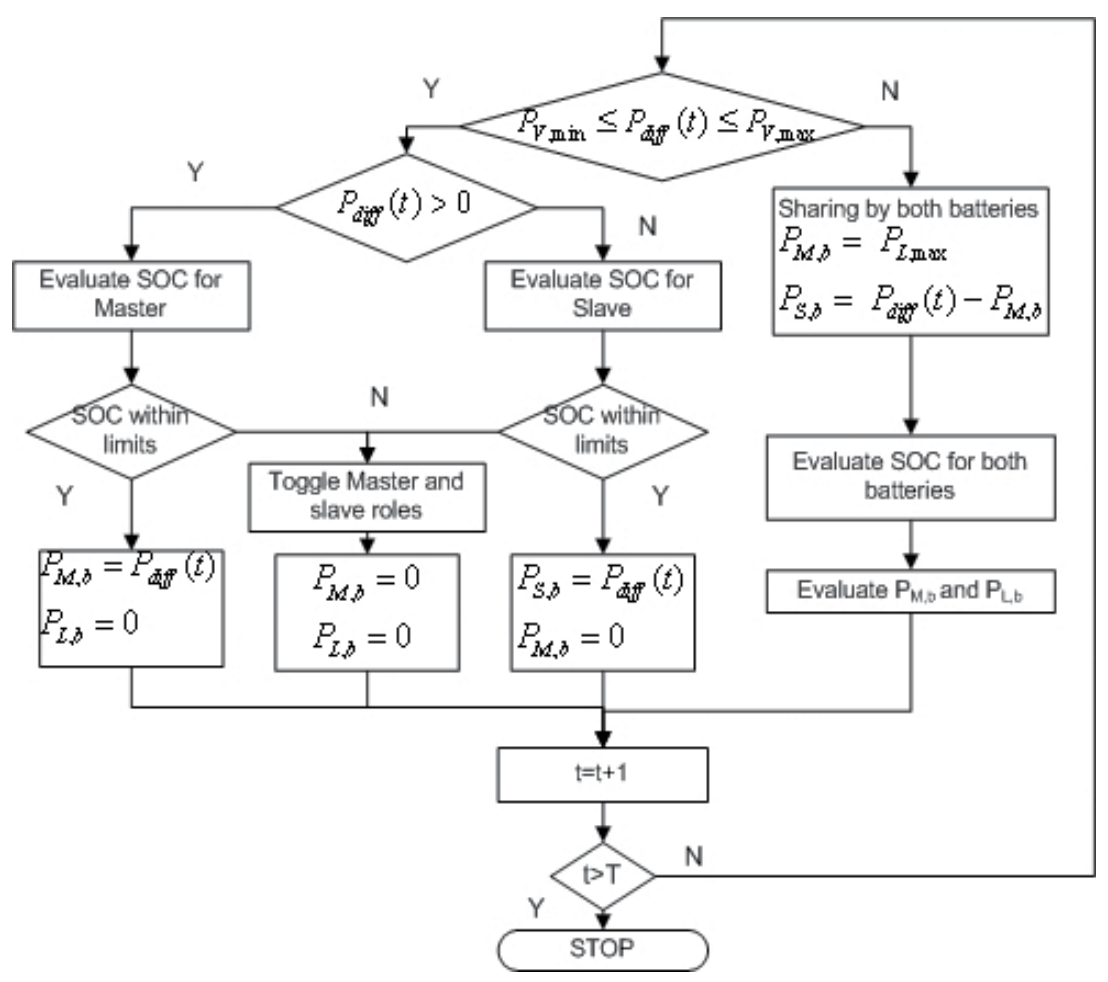

Figure 16. Energy management of dual VRB battery.

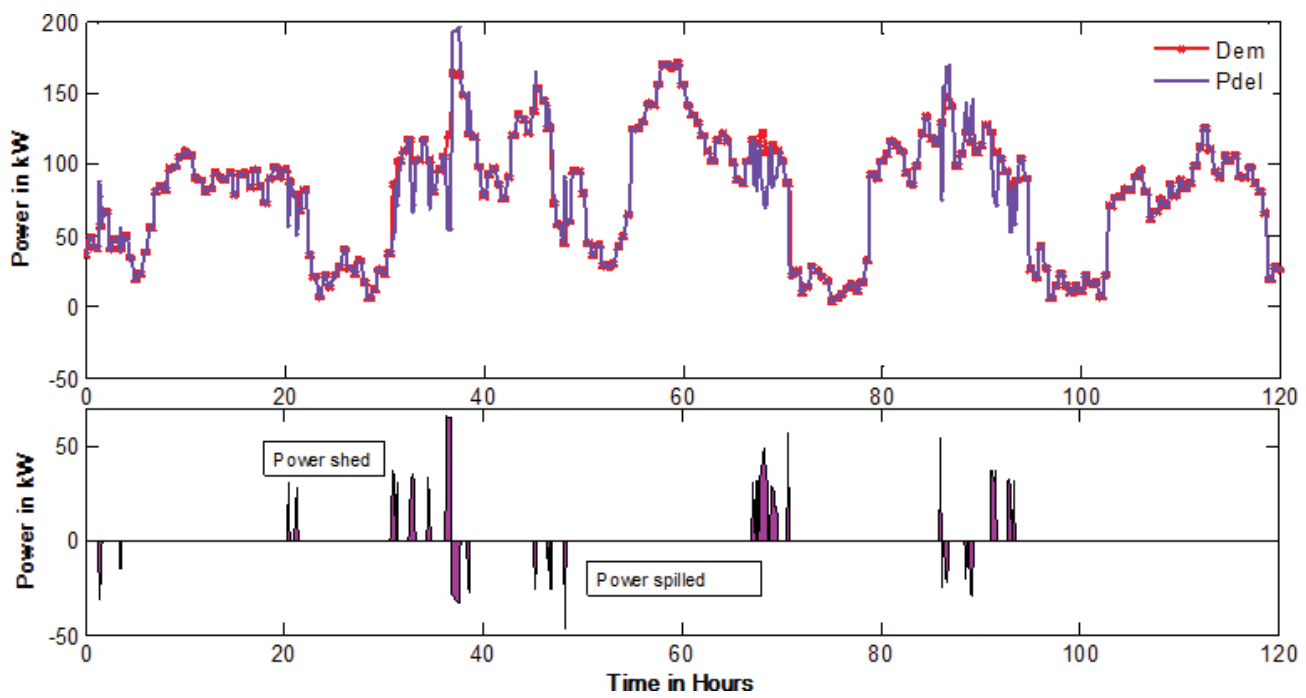

Figure 17. Power delivered curve (Dem vs $\left.P_{d i s}\right)$ and power mismatch $\left(\operatorname{Dem}-P_{d i s}\right)$. 


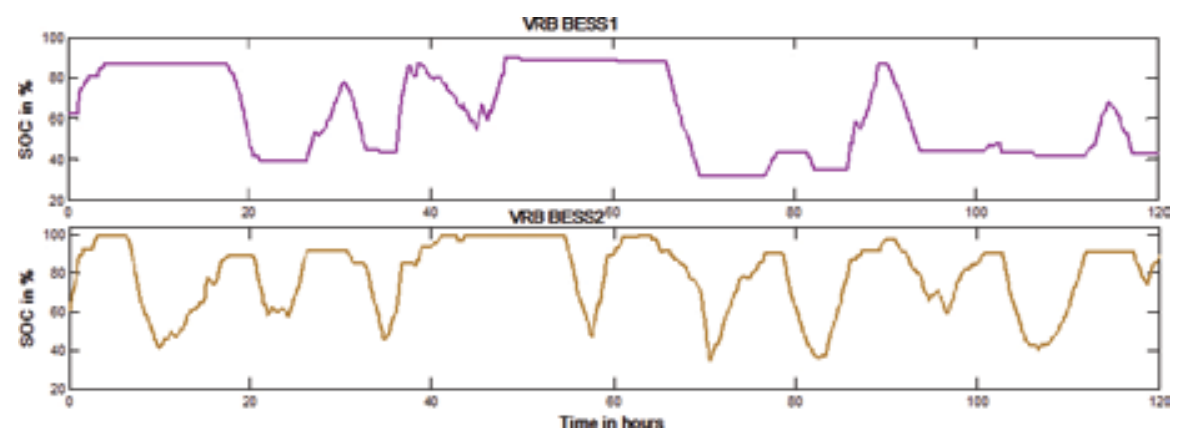

Figure 18. Dual VRB battery SOC.

The total energy delivered by the HRES is now increased to $23.3 \mathrm{MWh}$, and the shedding and spilling losses are reduced to 0.212 and $0.0925 \mathrm{MWh}$, respectively, resulting in a profit of $28,133 \$$ in the simulation period for 10 days. Assuming the VRB battery energy cost of about $600 \$ / \mathrm{kWh}$, the payback period is evaluated as about 4 years. The LPSP is now reduced to $1.21 \%$. Thus, the energy management of the energy storage has a great impact on the performance of the power system.

\section{Conclusion}

Energy storages are becoming indispensible for operation of DGs integrating renewable power sources. Advancement in technology now ensures power storage and delivery from few seconds to days/months. Optimized selection, sizing and siting of ESS will be critical for design engineers. Effective implementation and usage of ESS in the distributed grid requires intelligent and flexible energy management strategies capable of handling the dynamics of distributed systems. Most energy management systems focus on grid power balance and SOC of ESS. Recent research works focus on implementing energy management to minimize operating costs, manage uncertainties and reduce emissions. Application of optimization tools and techniques has enabled the development of flexible and effective energy management strategies. An effective dispatch and management strategy also needs to ensure efficient storage operation so as to enable its full life cycle usage. The challenge is to prioritize these objectives and evaluate a strategy most optimum for the considered application which can assure reliable power delivery without affecting system stability.

\section{Author details}

Amjed Hina Fathima* and Kaliannan Palanisamy

*Address all correspondence to: ahina.fathima2013@vit.ac.in

VIT University, Vellore, India 


\section{References}

[1] REN21 - Renewable Energy Policy Network for the 21st Century. The First Decade: 2004-2014 - 10 years of renewable energy progress. 2015.

[2] International Energy Agency. Distributed generation in liberalised electricity markets, 2002. 2002.

[3] Robert H. Lasseter. Microgrids and distributed generation. Intelligent Automation and Soft Computing. 2010;16(2): 225-234. DOI: 10.1080/10798587.2010.10643078.

[4] Purchala K., Belmans R., Exarchakos L., Hawkes A.D. Distributed generation and the grid integration issues. EUSUSTEL. https://www.eusustel.be/public/documents_publ/ WP/WP3/WP\%203.4.1\%20Distributed\%20generation\%20and\%20grid\%20integration \%20issues.pdf. Date accessed: 10/04/2016

[5] Lopes J.A.P., Moreira C.L., Madureira A.G. Defining control strategies for microgrids islanded operation. IEEE Transactions on Power Systems. 2006;21(2): 916-924.

[6] DOE. DOE Global Energy Storage Database [Internet]. [Updated: 26 October, 2015]. Available from: http://www.energystorageexchange.org/projects/data_visualization [Accessed: 4 February, 2016].

[7] Fathima A.H., Palanisamy K. Battery energy storage applications in wind integrated systems - A review. In: International Conference on Smart Electric Grid (ISEG), India; 19-20 September 2014. IEEE Xplore Digital Library. p. 1-8. DOI: 10.1109/ISEG. 2014.7005604.

[8] Gharavi H., Ardehali M.M., Ghanbari-Tichi S. Imperial competitive algorithm optimization of fuzzy multi-objective design of a hybrid green power system with considerations for economics, reliability, and environmental emissions. Renewable Energy 2015;78:427-437. DOI: 10.1016/j.renene.2015.01.029.

[9] Nykamp S., Bosman M.G.C., Molderink A., Hurink J.L, Smit G.J.M. Value of storage in distribution grids - Competition or cooperation of stakeholders? IEEE Transactions on Smart Grid. 2013;4(3):1361-1370. DOI: 10.1109/TSG.2013.2254730.

[10] Ipsakis D., Voutetakis S., Seferlis P., Stergiopoulos F., Elmasides C. Power management strategies for a stand-alone power system using renewable energy sources and hydrogen storage. International Journal of Hydrogen Energy 2009;34:7081-7095.

[11] Dash V., Bajpai P. Power management control strategy for a stand-alone solar photovoltaic-fuel cell-battery hybrid system. Sustainable Energy Technologies and Assessments 2015;9:68-80. DOI: 10.1016/j.seta.2014.10.001.

[12] Goebel C., Callaway D.S., Jacobsen H. The impact of state of charge management when providing regulation power with energy storage. IEEE Transactions on Power Systems. 2014;29(3):1433-1434. 
[13] Reihani E., Sepasi S., Roose L.R., Matsuura M. Energy management at the distribution grid using a battery energy storage system (BESS). Electrical Power and Energy Systems. 2016;77:337-344.

[14] Tewari S., Mohan N. Value of NAS energy storage toward integrating wind: Results from the wind to battery project. IEEE Transactions on Power Systems. 2013;28(1):532541.

[15] Díaz-González F., Sumper A., Gomis-Bellmunt O., Bianchi F.D. Energy management of flywheel-based energy storage device for wind power smoothing. Applied Energy 2013;110:207-19.

[16] Chandra S., Gayme D.F., Chakrabortty A. Coordinating wind farms and battery management systems for inter-area oscillation damping: A frequency-domain approach. IEEE Transactions on Power Systems. 2014;29(3):1454-1462.

[17] Zhou K., Ferreira J.A., de Haan S.W.H. Optimal energy management strategy and system sizing method for stand-alone photovoltaic-hydrogen systems. International Journal of Hydrogen Energy 2008;33:477-489.

[18] García P., Torreglosa J.P., Fernández L.M., Jurado F. Optimal energy management system for standalone wind turbine/photovoltaic/hydrogen/battery hybrid system with supervisory control based on fuzzy logic. International Journal of Hydrogen Energy 2013;38:14146-14158.

[19] García P., Torreglosa J.P., Fernández L.M., Jurado F, Langella R., Testa A. Energy management system based on techno-economic optimization for microgrids. Electric Power Systems Research 2016;131:49-59.

[20] Cheng Y., Chuang M., Liu Y., Wang S., Yang Z. A particle swarm optimization based power dispatch algorithm with roulette wheel re-distribution mechanism for equality constraint. Renewable Energy 2016;88:58-72.

[21] Wang C., Liu Y., Li X., Guo L., Qiao L., Lu H. Energy management system for standalone diesel-wind-biomass microgrid with energy storage system. Energy 2016;97:90-104.

[22] Mohammadi S., Mozafari B., Solimani S., Niknam T. An adaptive modified firefly optimisation algorithm based on Hong's point estimate method to optimal operation management in a microgrid with consideration of uncertainties. Energy 2013;51:339-348.

[23] Mohammadi S., Soleymani S., Mozafari B. Scenario-based stochastic operation management of microgrid including wind, photovoltaic, micro-turbine, fuel cell and energy storage devices. International Journal of Electrical Power and Energy Systems. 2014;54:525-535.

[24] Mohammadi S., Mozafari B., Solimani S., Niknam T. Stochastic scenario based model and investigating size of ESS for PEM-fuel cell unit commitment for microgrid consid- 
ering profitable strategies. IET Generation, Transmission and Distribution 2014;8(7): 1228-1243. DOI:10.1049/iet-gtd.2013.0026.

[25] Chaouachi A., Kamel R.M., Andoulsi R., Nagasaka K. Multiobjective intelligent energy management for a microgrid. IEEE Transactions on Industrial Electronics. 2013;60(4): 1688-1699.

[26] Chen Y., Wu Y., Song C., Chen Y. Design and implementation of energy management system with fuzzy control for DC microgrid systems. IEEE Transactions on Power Electronics. 2013;28(4):1563-1570.

[27] Feroldi D., Rullo P., Zumoffen D. Energy management strategy based on receding horizon for a power hybrid system. Renewable Energy 2015;75:550-559.

[28] Palma-Behnke R., Benavides C., Lanas F., Severino B., Reyes L., Llanos J., DorisSáez A. Microgrid energy management system based on the rolling horizon strategy. IEEE Transactions on Smart Grid. 2013;4(2):996-1006.

[29] Shu Z., Jirutitijaroen P. Optimal operation strategy of energy storage system for gridconnected wind power plants. IEEE Transactions on Sustainable Energy. 2014;5(1): 190-199.

[30] Gabash A., Li P. Active-reactive optimal power flow in distribution networks with embedded generation and battery storage. IEEE Transactions on Power Systems. 2012;27(4):2026-2035.

[31] Fu Q., Montoya L.F., Solanki A., Nasiri A., Bhavaraju V., Abdallah T., Yu D.C. Microgrid generation capacity design with renewables and energy storage addressing power quality and surety. IEEE Transactions on Smart Grid. 2012;3(4):2019-2027.

[32] Silva-Monroy C.A., Member IEEE, Watson J. Integrating energy storage devices into market management systems. Proceedings of the IEEE. 2014;102(7):1084-1093.

[33] Abdeltawab H.H., Mohamed Y.A.R.I. Market-oriented energy management of a hybrid wind-battery energy storage system via model predictive control with constraint optimizer. IEEE Transactions on Industrial Electronics. 2015;62(11):6658-6670.

[34] Fathima A.H., Palanisamy K. Optimized sizing, selection, and economic analysis of battery energy storage for grid-connected wind-PV hybrid system. Modelling and Simulation in Engineering 2015. vol.2015, 16 pages Article ID 713530. DOI: $10.1155 / 2015 / 713530$. 

Chapter 8

\title{
Energy Storage Technology for Decentralised Energy Management: Future Prospects
}

\author{
Bartek A. Glowacki and Emma S Hanley \\ Additional information is available at the end of the chapter \\ http://dx.doi.org/10.5772/63415
}

\begin{abstract}
The chapter provides a comparison of energy storage technologies in decentralised energy systems for energy management. The various costs, advantages and disadvantages of the storage technologies will be considered. System dynamics modelling will be used to analyse energy management within the decentralised renewable and storage systems. Additionally, the integration of hydrogen storage technology and the use of hydrogen as an energy carrier in a decentralised airport scenario will be highlighted and the arising advantages of a decentralised airport using novel electric planes powered by hydrogen are discussed.
\end{abstract}

Keywords: decentralised energy storage, energy management, transport, hydrogen, airbus

\section{Introduction}

Successful management of future energy systems requires not efficient generation and use of energy but also the integration of storage technology to improve energy security, reduce fuel price volatility and allow further penetration of renewable energy by managing energy generation. There are many different types of storage technologies and approaches available withredoxflowbatteries(RFBs)andhydrogenstoragebeing discussed infurtherdetailincluding current and future techno-economic impacts of the storage technology. The use of storage technologies is of paramount importance for transitioning to a low-carbon, sustainable and resource efficient economy. The potential integration of energy storage technologies can be complementary within systems for optimal energy management and will also be considered within the study. 
The subject area of this chapter will focus on the energy management of decentralised energy systems using storage technologies. As a result of the transitioning energy sector towards a decarbonised system, a lot of change is occurring within the industry. The development and use of energy storage technologies are one such change that is occurring. Energy storage devices can manage the supply and demand mismatch of renewable energy within decentralised systems. The anticipated hydrogen economy will be of focus as one sustainable energy carrier for storage and therefore energy management. Both compressed and liquid hydrogen will be considered as hydrogen is important as it allows the storage of an energy carrier that can also be used as a cryogen when liquefied which will have implications for the superconducting industry. This additional benefit of hydrogen for will be considered in further detail with the use of liquid hydrogen for a decentralised future innovative airport scenario highlighted.

The preliminary chapter map is presented in Figure 1. The growth of renewable energy will be first discussed. Although the continued integration of renewable energy increases indigenous energy generated and therefore reduces import dependence. It is important to note with increased intermittent energy generation introduced an increased amount of back-up fossil fuel energy or adequate amounts of storage capacity is required. Storage technology with focus on hydrogen and redox flow batteries will then be considered. Finally, the case for the decentralised hydrogen production, storage, liquefaction and use on electric airplanes will be presented.

\section{Energy (Renewable Energy)}

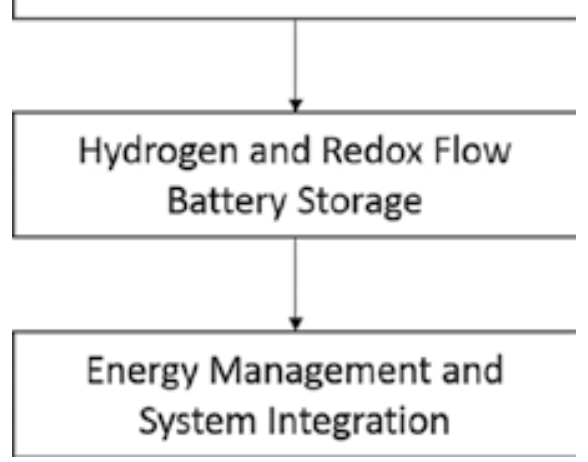

Figure 1. Preliminary chapter map.

The key result will present decentralised hydrogen and redox flow batteries for storage that can be used for energy management. The study will provide a basis for reference when considering the current and future prospects of energy storage in decentralised energy systems that can aid with the management of renewable energy. Further advantages and disadvantages of the technologies will be considered also including additional benefits arising from storage 
focusing on the storage of hydrogen as an energy carrier for novel electrically powered, superconducting airplanes.

\section{Renewable energy and energy storage}

\subsection{Growth of renewable energy}

The world is transitioning to a decarbonised economy, less than 300 years after the emergence of the industrial revolution. There is widespread acceptance for this transition due to accelerating climate change, increasing population and increasing demand for finite resources. A shift towards the use of novel, low carbon alternative fuels and technology is imminent. As from Table 1, it can be deduced an overall electricity demand is increasing; however, more of this electricity is being met by renewables. Additionally, renewable energy is also important for the heat and transport sectors and it is estimated that $11 \%$ of energy consumption is from renewable energy sources and this is expected to rise to $15 \%$ by 2040 [1]. With the continued penetration of renewable energy storage technology can be a potential solution to manage curtailment within the system caused by supply and demand mismatch.

\begin{tabular}{lll}
\hline & $\mathbf{2 0 0 0}(\mathbf{\%})$ & $\mathbf{2 0 1 2}(\mathbf{\%})$ \\
\hline Nuclear & 16.68 & 10.86 \\
Fossil Fuels & 63.57 & 67.17 \\
Hydroelectricity & 17.86 & 16.89 \\
Geothermal & 0.351 & 0.315 \\
Solar & 0.007 & 0.444 \\
Tide and Wave & 0.004 & 0.002 \\
Wind & 0.21 & 2.41 \\
Biomass and Waste & 1.12 & 1.78 \\
Pumped Hydroelectric Storage & 0.18 & 0.12 \\
Total Electricity (billion $\mathrm{kWh})$ & 14681.87 & 21582.97 \\
\hline
\end{tabular}

Table 1. Global electricity generation expressed as a percentage of total electricity generation [2].

\subsection{Energy storage}

Storage systems like pumped hydroelectric energy storage (PHES) have been in used since 1929 for energy management [3]. Although it is clear that energy storage is an established concept, storage technologies are currently not a widespread solution. Energy storage technologies have different characteristics including applications, suitable power capacities, energy storage capacities, efficiencies, costs and response time. A discussion on the integration of energy storage technologies to complement other storage technologies will be included. The main function of the discussion of storage systems is to identify their role in energy manage- 
ment of decentralised energy generation systems integrated with renewable technology. Furthermore, storage technology is important within energy systems as it can serve many different functions that will be further discussed in the case of hydrogen. There are a wide range of storage technology that are available today; however, many are not currently at the commercial stage or suffer from high economic costs. Currently, pumped hydroelectric energy storage represents $98.3 \%$ of total installed storage capacity for the grid ( $127 \mathrm{GW})$ and less than $10 \mathrm{MW}$ of capacity is from redox flow batteries, Figure $2[4,5]$. The use of alternative energy storage technologies to pumped hydro-electric storage can allow the continued successful integration of renewable energy into the grid. Renewable energy allows countries to develop an indigenous energy supply as resources are available worldwide.

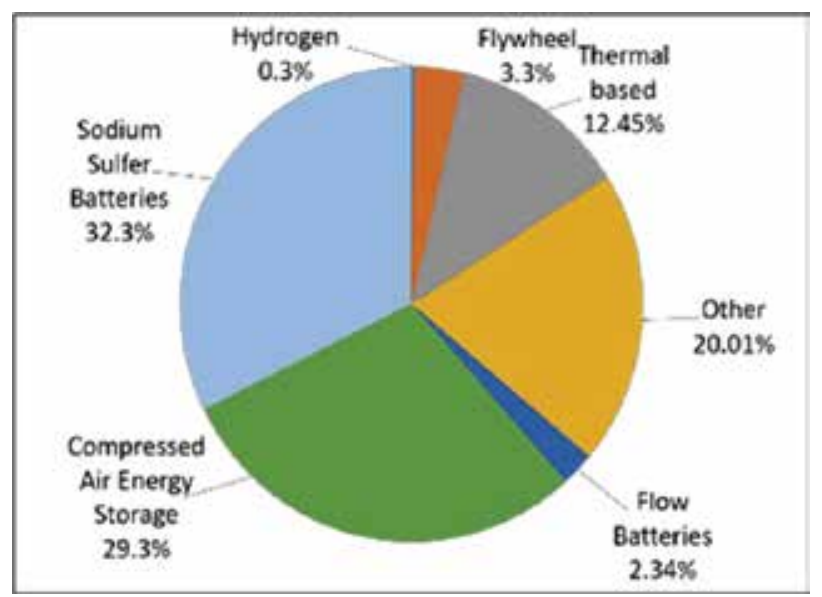

Figure 2. Non-pumped hydroelectric storage installed capacity accounting for $1 \%$ of worldwide storage capacity, with hydrogen and flow batteries included [4].

As the energy industry is undergoing a transition to a decarbonised energy system, energy storage is becoming a realistic option to aid this transition. Hydrogen storage and redox flow batteries are further discussed in the next section.

In an energy view depicted in Figure 3, the use of storage including hydrogen storage, pumped hydroelectric storage and stationary battery storage is considered. However, the use of the stored energy is considered only for electricity and meeting electric needs in a centralised manner. This chapter wants to provide an insight into the management of distributed energy systems that can focus more on the overall picture rather than just electricity. The use of renewable energy within the energy system has mainly focused on the electricity sector. Currently, in the European Union, $25.5 \%$ of electricity demand is met by renewables, $16.5 \%$ for heat and cooling and 5.4\% for transport [6]. The focus for the use of renewable energy for transport will increase as a result of energy polices and energy security particularly in the transport sector. The source of final energy consumption is becoming more important, and the need for more complex energy systems that integrate the electricity, heat and transport sectors is required to ensure the optimal management and use of resources. Hydrogen is a flexible 
energy carrier as a result its potential use in the sectors as mentioned and alternatively as a storage and cryogenic medium.

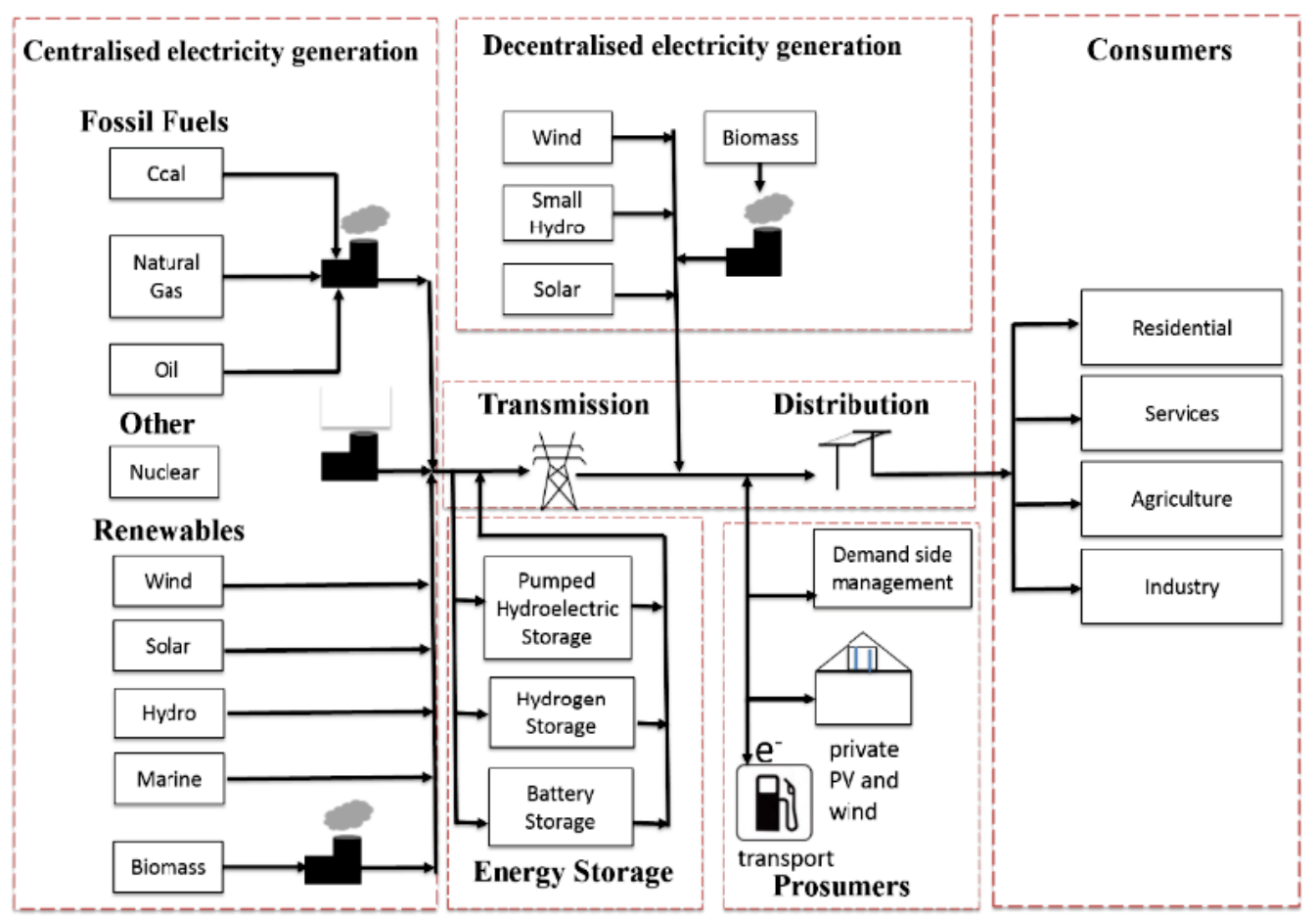

Figure 3. Current energy systems highlighting the dependence on centralised energy generation.

\section{Hydrogen and redox flow batteries storage technologies}

\subsection{Hydrogen storage for energy management}

Hydrogen is one sustainable alternative fuel and cryogen for future energy and resource requirements that can be stored in both gaseous and liquid form. Hydrogen's use as an energy carrier is well known; however, it has failed to successfully penetrate energy markets on a large scale. With focus on the transition from conventional energy generation methods and fuels, the 'hydrogen economy' can now emerge and be a key enabler to securing a sustainable, decarbonised energy future [7-9].

For hydrogen to be considered, a low-carbon fuel renewable electrolysis and zero-low carbon methods of hydrogen production using natural gas such as the microwave plasma processing of natural gas and thermal cracking of methane can be considered for a decentralised solution. The cost of hydrogen from wind electrolysis depends on the wind electricity generation price in a particular region, but it can typically vary from 3.58 to $5.86 \$ / \mathrm{kg}$ with other sources 
estimating higher costs of 6-7 \$/kg [10]. PV electrolysis is more expensive than wind electrolysis with expected current values of $28.19 \$ / \mathrm{kg}$ and future values of $6.18 \$ / \mathrm{kg}$ due to expected rapid cost decrease of PV energy [10,11]. Among different processing methods, microwave plasma processing of natural gas is a 'low-emission' (zero $\mathrm{CO}_{2}$ ) production method. The technology has a high efficiency with an estimated hydrogen production cost of $1.5 \$ / \mathrm{kg}$, noticeably lower than the renewable electrolysis process, and is dependent on natural gas prices. Alternatively, the steam methane reforming method of hydrogen production the most common way to produce hydrogen today integrated with carbon capture and storage can be considered [12]. Low-carbon hydrogen generation is anticipated due to the aforementioned increase in penetration of renewable energy and also for providing an additional low-carbon fuel for the transport sector. Therefore, suitable methods for bulk energy storage and on-board storage for hydrogen transport must be available [13]. Four different methods of hydrogen storage are currently being considered; high pressure compressed hydrogen, liquid hydrogen in insulated tanks, solid-state hydride storage and porous solid adsorption of molecular hydrogen [14, 15]. Storage of compressed hydrogen requires high pressures (200-700 bar) and liquid hydrogen requires low temperatures (20.39 K) [16]. Another possibility for storing hydrogen is by the formation of metal hydrides. High volumetric capacities can be reached with metal hydrides, but energy is required for heating for hydrogen release. Finally, adsorption in porous material is an alternative hydrogen storage method that research has grown significantly.

Carbon fibre-reinforced composite tanks for 350 bar and 700 bar compressed hydrogen are under development and are already used for hydrogen storage for stationary applications and hydrogen-powered vehicles. The cost of high-pressure compressed hydrogen gas tanks depends on the pressure needed and the amount of the carbon fibre that must be used for structural reinforcement for the storage tanks. Liquid hydrogen is an alternative hydrogen storage method. A hybrid liquid hydrogen storage and superconducting magnetic energy storage (SMES) system can provide a robust energy system for back-up power. Alternatively, it can be considered for storage at refuelling stations for transport [14]. Liquid hydrogen tanks can, in principle, store more hydrogen in a given volume than compressed gas tanks, since the density of liquid hydrogen is $70 \mathrm{~kg} / \mathrm{m}^{3}$ compared to compressed hydrogen that has a density of $39 \mathrm{~kg} / \mathrm{m}^{3}$ at 700 bar, Figure 4 [13].

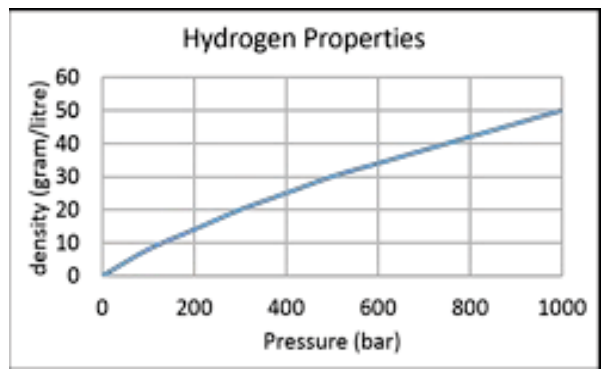

Figure 4. Increasing density of hydrogen with pressure for compressed hydrogen storage [13]. 
Liquid hydrogen is stored in cryogenic tanks at $\sim 20 \mathrm{~K}$ at ambient pressure because of the low critical temperature of hydrogen $(33 \mathrm{~K})$ [17]. Key issues with liquid hydrogen tanks are hydrogen boil-off estimated at 1\%/day [14], and the large amount of energy required for hydrogen liquefaction [14], as well as tank cost [13]. Liquid hydrogen storage has the largest energy requirement and for storage times longer than a week the boil-off rate is problematic. For compressed hydrogen, the storage cost is eventually limited by the compressor electricity cost. One option for compressed gas storage is to increase the operating pressure of the system. This increases the cost of the pressure vessel and compressor, but the reduction in tank size can result in an overall savings [18]. The hydrogen stored can be used in a wide range of energy management techniques discussed in the next section.

Hydrogen is envisioned to emerge in niche decentralised markets and can be used for energy management of renewable energy as well as the use in transport. In this sense, hydrogen could form the basis of a synergistically operating buffer mechanism facilitating the integration of intermittent renewable energy, reducing $\mathrm{CO}_{2}$ emissions as well as enhancing indigenous energy supply and increasing energy security. For the investigation of the hydrogen buffer operation if an unconstrained system using surplus renewable electricity during low demand hours for hydrogen generation and storage is considered the system may result in hydrogen not being produced if there is no excess wind. This would mean a lack of energy security within the system. Alternatively, the use of a hydrogen buffer system for energy management that constrains the wind energy for hydrogen production instead of demand to provide some security to the system could be alternatively considered, Figure $5 \mathbf{b}$ as a solution.

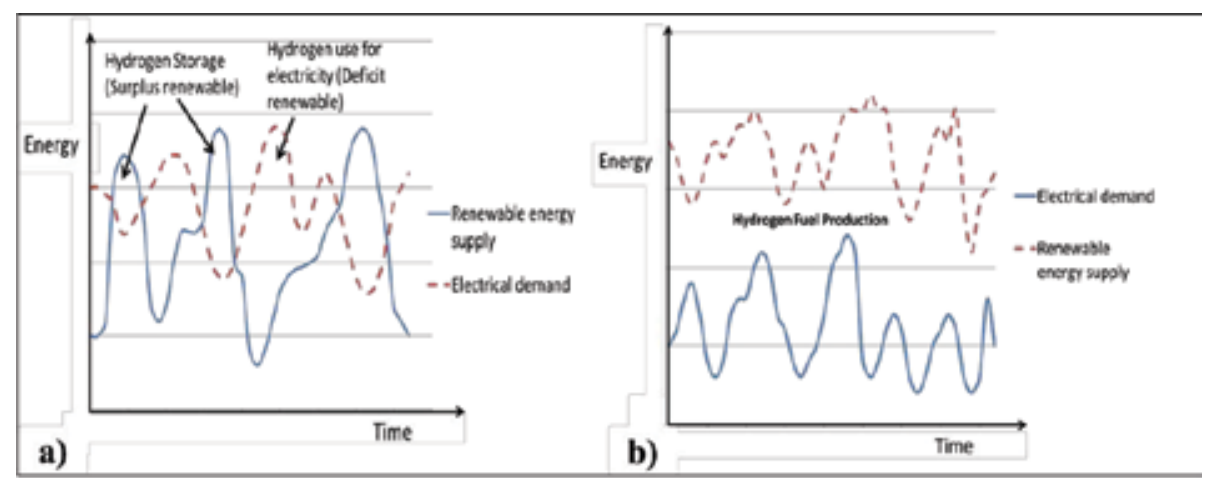

Figure 5. Comparison of (a) hydrogen storage for meeting demand when required leaving the system vulnerable to a lack of hydrogen energy available in storage, (b) hydrogen use as a buffer allowing excess hydrogen to be accessed if required.

System dynamics is a system modelling tool that uses various control factors and observes how the system and variables behave in response to time-based trends. In system dynamic models, there are main stock and flow quantities. Stocks represent the status of the system, the quantities that exist at any given moment (e.g. hydrogen storage). Rate variables show the speed of flow in or out of the stocks (e.g. hydrogen production and use), and they serve as the 
decision making variables in a system. From a system dynamics model, the cost of electricity calculated varies from 0.4 to $0.97 € / \mathrm{kW}$ h when the system is ran with no energy buffer, Figure 6. Although with optimum cost for the high wind scenario, this system is vulnerable to a large increase in the price with low wind energy. With this operation, the hydrogen production and use are not managed. When there is extra wind in the system, hydrogen is produced; when there is a deficit of energy within the system, hydrogen is converted to electricity (Figure $\mathbf{5 b}$ ). Figure $5 \mathbf{b}$ shows the operation of a hydrogen buffer with increased security in the system with the hydrogen storage acting as a buffer for the wind energy. The system is managed and constrained to ensure that hydrogen is available if there is now renewable energy available in the system. In the system that constrains, the use of hydrogen for peak times only the cost of electricity from hydrogen ranges from 0.74 to $0.85 € / \mathrm{kW} \mathrm{h}$. The estimated cost of electricity from hydrogen ranges from 0.28 to $0.6 € / \mathrm{kW}$ h in literature [19]. The results highlight the potential use of a hydrogen buffer storage system to manage decentralised renewable energy systems.

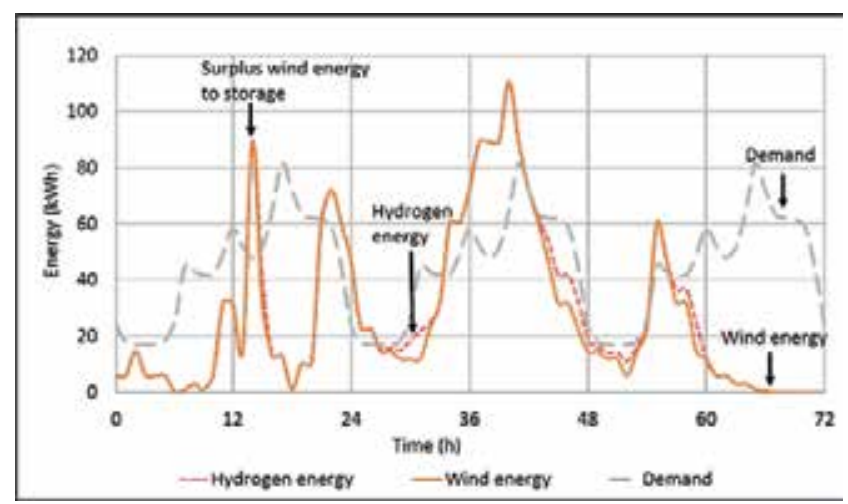

Figure 6. Energy versus time diagram for system operation without energy management of hydrogen storage as a buffer. Excess wind energy produces hydrogen for storage; however, not enough hydrogen is available to prevent the requirement of grid energy but reduces curtailment in the system.

\subsection{Redox flow batteries}

Redox flow batteries (RFBs) have promising storage characteristics and, as the power and energy capacity of the battery are independent of each other, the RFBs can be optimised to maximise the performance and minimise the cost [20,21]. RFBs are rechargeable systems that have the storage medium in the form of electrolyte kept in tanks external to the active cell. The electrochemical reactions and the charging and discharging battery cycles are taking place in the battery stack as the electrolyte flows through the two membrane-separated chambers of the active cell, Figure 7 [20,21]. The energy is stored in the separated reactants (electrolytes), while the power is controlled by the stack, Figure 7 [20, 21]. In general, RFBs share similar flow geometries and the main differences typically occur in the electrolyte that is used [21-23]. The RFBs can operate at low temperatures (from -10 to $+45^{\circ} \mathrm{C}$ ) as long as the electrolytes remain stable and their precipitation does not occur. 


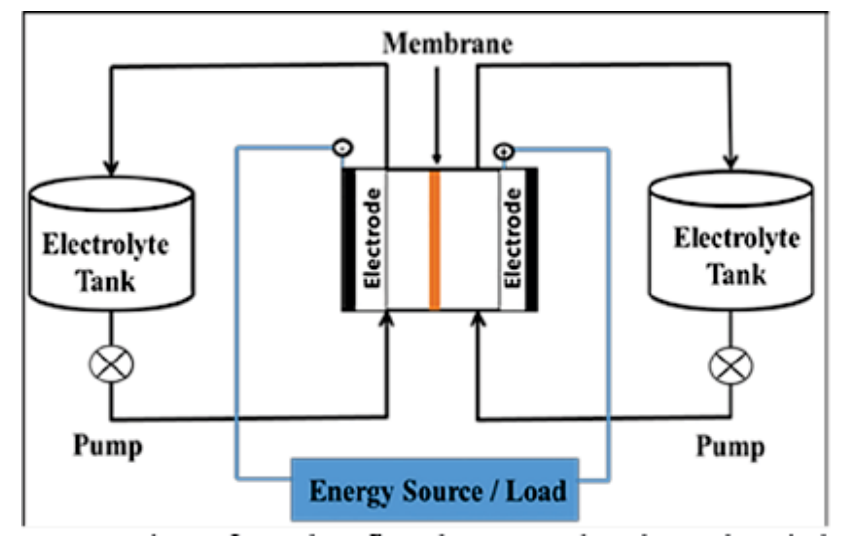

Figure 7. Schematic representation of a redox flow battery. The electrolyte is kept in outside tanks and pumped in the two membrane-separated chambers of the active cell.

\subsubsection{Vanadium redox flow batteries}

The vanadium redox flow batteries (VRFBs) have promising energy storage characteristics and can respond to unpredictable changes in wind speed. The VRFB has a high efficiency in the range of $65-80 \%$, but it has a relatively low energy density and this represents one of the main disadvantages [24-26]. The theoretical energy density is 30-47 Wh/l, but the practical achievable energy density is lower at $15-25 \mathrm{Wh} / 1$ [26]. When storage capacity needs to be increased, the low energy density leads to large electrolyte volumes. The electrolyte is evenly split in VRFB between the positive and negative tanks. The reactions that occur within the cell during charging, and discharging cycles are shown in Table 2.

\begin{tabular}{lll}
\hline & VRFB & All-Iron RFB \\
\hline Positive side & $\mathrm{VO}^{2+}+\mathrm{H}_{2} \mathrm{O}-\mathrm{e}^{-} \rightarrow \mathrm{VO}_{2}^{+}+2 \mathrm{H}^{+}$ & $\mathrm{Fe}^{2+} \leftrightharpoons \mathrm{Fe}^{3+}+\mathrm{e}^{-}$ \\
Negative side & $\mathrm{V}^{3+}+\mathrm{e}^{-} \leftrightharpoons \mathrm{V}^{2+}$ & $\mathrm{Fe}^{2+}+2 \mathrm{e}^{-} \leftrightharpoons \mathrm{Fe}^{0}$ \\
\hline
\end{tabular}

Table 2. The chemical reactions occurring at the negative and positive side of the VRFB and all-iron RFB.

There are several advantages of using VRFB for energy storage applications: long cycle life (>10,000 cycles), high reliability, deep discharge capability and high power density. Although the electrodes do not store energy, they are important for charging and discharging of the battery, influencing, together with the electrolyte and separation membrane, the life-time of the battery, the energy losses and, consequently, the overall efficiency. It is anticipated that efficiency improvements can be made with regard to the correct selection of electrodes, for example using carbon black or its activated composites [27]. Other advantages include the popularity of the battery with regard to research and also the many VRFB installations worldwide. 


\subsubsection{All-iron redox flow batteries}

The all-iron RFB like VRFB employs the use of a single chemical element (in this case iron) in several oxidation states on both sides of the active cell, Table 2, while the electrolyte is kept outside in the storage tanks. The positive electrode of the all-iron battery is the ferric/ferrous redox couple, and the negative electrode involves iron plating from Fe (II) [22]. An advantage of all-iron RFB is the readily available electrolyte with an estimated low cost of $0.23 \$ / 1$ [23]. In the traditional all-iron RFB, at the negative side, the ferrous ions are reduced during charge. Their plating as iron metal onto a graphite electrode of the stack occurs leading to a coupling between energy and power. On the positive side of the battery, ferrous ions are oxidised to ferric ions during charge remaining in the solution. Reactions are opposite on discharge. Cheap aqueous electrolytes, inexpensive separators and the widespread availability of iron $(\sim 230$ billion metric tonnes of iron) give the all-iron RFB, the potential of reduced storage system cost, while the plating and, consequently, the coupling between the energy and power represents its main disadvantage [22, 23].

To avoid this disadvantage, a slurry electrode containing electrically conductive carbonaceous particles can be made by flowing them in an electrolyte containing the dissolved iron species [22]. Such conductive particles can include carbon black and/or carbon allotropes with different surface areas and enhanced conductivity, carbon micro-flakes, nanofibres, nanotubes etc. Thus, iron is plated onto the carbon particles at the negative side while charging. The carbon particles can then carry the iron metal to be stored in the external tanks allowing for energy storage capacity and power decoupling, allowing the economic advantages of scaling inherent to RFB to be recovered [22]. The carbons and their properties influence the electronic conductivity of the slurry electrodes that have to be greater than the ionic conductivity of the electrolyte. This allows for the iron deposition to occur only onto the slurry particles and not on the current collector leading to a better control of the current distribution [22]. The electrode surface area plays an important role in determining the all-iron (hybrid) RFB efficiency and lifetime. For slurry all-iron RFB, this role becomes secondary. Presently, the slurry all-iron RFBs are still in the development stage putting them at a disadvantage to the already commercialised VRFB. Typically, the energy density of the all-iron hybrid battery is $12.7 \mathrm{Wh} / \mathrm{l}$ with a specific energy $10.9 \mathrm{Wh} / \mathrm{kg}$. Energy efficiency is $55 \%$ with operating temperature $\mathrm{T}_{0}=40^{\circ} \mathrm{C}$ $[22,27]$.

\subsubsection{Energy storage integration}

There is much focus on the introduction of storage systems; however, the integration of different storage systems to complement the use should also be considered. For example, research was conducted on the integration of compressed hydrogen storage and VRFB. From system dynamics modelling, it was identified as a result of higher available wind energy for storage and the hydrogen system is able to provide more energy to the system due to its capability as a bulk energy storage medium. In contrast, the RFBs are capable of providing more energy to the system with reduced availability of wind energy for storage, as a result of higher efficiencies. Therefore, VRFBs or all-iron RFBs are more efficient energy storage at times when there are no high periods of curtailment. 
The storage systems benefit from increased value regarding technical and economic factors when integrated with other complementary energy storage technology [28]. This is as a result of the capability of hydrogen for bulk energy storage and VRFB with higher efficiencies is complementary. Furthermore, the effect of the integrated systems depends on the level of excess wind sent to either the hydrogen or VRFB storage system. In independent systems, all the excess wind is sent to each individual storage technology; however, with an integrated approach, the excess wind must be split between the two storage systems.

Storage systems benefit from increased value regarding technical and economic factors when integrated with other complementary energy storage technology.

\section{Decentralised hydrogen airport scenario}

\subsection{Decentralised energy systems}

Decentralised energy systems are gaining focus due to energy security and climate change considerations along with the high GHG emissions from centralised fossil fuel plants. Decentralised energy systems can potentially allow for the changes required in the energy sector [2936]. Advantages include their ability to operate with more than one source of energy and also their potential to be integrated with renewable energy and storage systems [33, 34]. Currently, the majority of energy systems consist of centralised power plants. Centralised energy generation benefits from high economies of scale, base load power capacity and reliability (if energy resources are available) [35]. However, it is clear a transition from these conventional fossil fuel power plants is required. Challenges of decentralised energy include technical challenges of operating the power plants and reliability of the overall system as if the power plant is relying on non-dispatchable generation, the capacity can be affected and require investments in back-up power [33].

Energy management is required for planning energy generation for consumption. Energy management is important for mitigating energy problems. It allows for the optimum operation of energy generation and storage systems to maximise efficiency. It is evident that within renewable decentralised systems, energy storage and energy management of these systems will play an important role. The complexity of the integration of the systems will require management to optimise the generation, storage and use of energy. Decentralised energy systems are envisioned for a hydrogen economy to emerge. Both compressed and liquid hydrogen energy systems can provide valuable green energy carrier if produced from zero/low carbon emission methods.

The next section will further highlight the different applications and importance of liquid hydrogen with a discussion on the decentralised use of liquid hydrogen in an airport scenario.

\subsection{Hydrogen as a cryogen}

An additional application for liquid hydrogen $(20 \mathrm{~K})$ is as a cryogen for superconducting technologies. Interest has grown in finding a suitable low temperature cryogen as a result of 
predicted helium shortages and price increases. There is a predicted and well-documented incoming shortage of helium for superconducting applications [37-42],] and hydrogen as a cryogenic coolant has been envisaged as a viable and more economically justified cooling option for superconducting devices [37]. There are many novel engineering designs that can be made possible by using medium-temperature $\mathrm{MgB}_{2}$ superconducting wires, as developed originally in Cambridge [43] that include the following; a self-contained fully electric superconducting ship, DC fault current limiters, high DC current homopolar motors, cheaper superconducting $\mathrm{MgB}_{2}$ magnets for fusion [41], SMES [41-43] and MRI systems. Development of liquid hydrogen indirectly cooled $\mathrm{MgB}_{2}$ superconducting high voltage DC cables especially for computer data centres present ideal candidates for early implementation [44]. Hydrogen's use as a coolant, as well as an energy carrier, will spin off new research and developments in superconducting materials and efficient energy use.

As the quantity of hydrogen liquefied is increased, less energy is wasted and the more efficient and cost-effective the process. The liquefaction process can occur by the Joule-Thomson expansion cycle. The hydrogen is compressed at ambient pressure and passed through a heat exchanger in which the temperature is reduced. As a result of hydrogen cooling on expansion, the temperature should be below the inversion temperature $T_{\mathrm{inv}}=200 \mathrm{~K}$. A nitrogen precooling step is introduced, before the hydrogen is passed to the expansion valve. The energy required for the compressor and expansion valves reduces the overall efficiency of the process. As liquid hydrogen is a cryogen with a low boiling temperature of $T_{\text {boil }}=20 \mathrm{~K}$ (under normal pressure), it must be stored in insulated cryogenic containers which are designed with double walls and an insulating space between the two walls to reduce heat transfer to the liquid. Heat transfer causes the liquid to evaporate and form gas a process called boil-off. Heat also arises from the ortho-para conversion of hydrogen. To minimise boil-off of the hydrogen for longer storage, an ortho-para conversion must be completed before liquefaction. The use of catalysts facilitates the ortho-para conversion of hydrogen [45].

Considering liquid hydrogen safety, direct cooling can only be handled by highly specialised organisations and companies, but indirect liquid hydrogen cooling, $\left(\mathrm{iLH}_{2}\right)$, can be a viable option. In $\mathrm{iLH}_{2}$ installations, a helium gas exchanger can be used, transferring cooling power of the hydrogen bath at $20 \mathrm{~K}$ to the desired cryomagnetic installation [45]. A pertinent example of indirect cooling by liquid nitrogen is given by McDonald et al. that designed a cooling system for a $15 \mathrm{~T}$ pulsed copper solenoid magnet to a desired temperature of $30 \mathrm{~K}$ in order to reduce the resistance of the $\mathrm{Cu}$, thereby reducing the power requirements of the system [45]. The design as proposed cooled the magnet via a closed helium loop circulated through a heat exchanger filled with liquid hydrogen from a storage Dewar. It is clear hydrogen both compressed and liquefied will have important implications for different aspects of energy systems.

\subsection{Airport scenario}

As a further development of hydrogen storage, a decentralised vision of low-carbon airport systems will be analysed. Low-carbon systems integrated with hydrogen will be important as a result of the increasing threat of climate change, resource consumption and increasing energy 
demand. Storage systems alone will not be able to solve these problems, and innovative solutions integrated with storage systems are required such as that depicted in Figure 8.

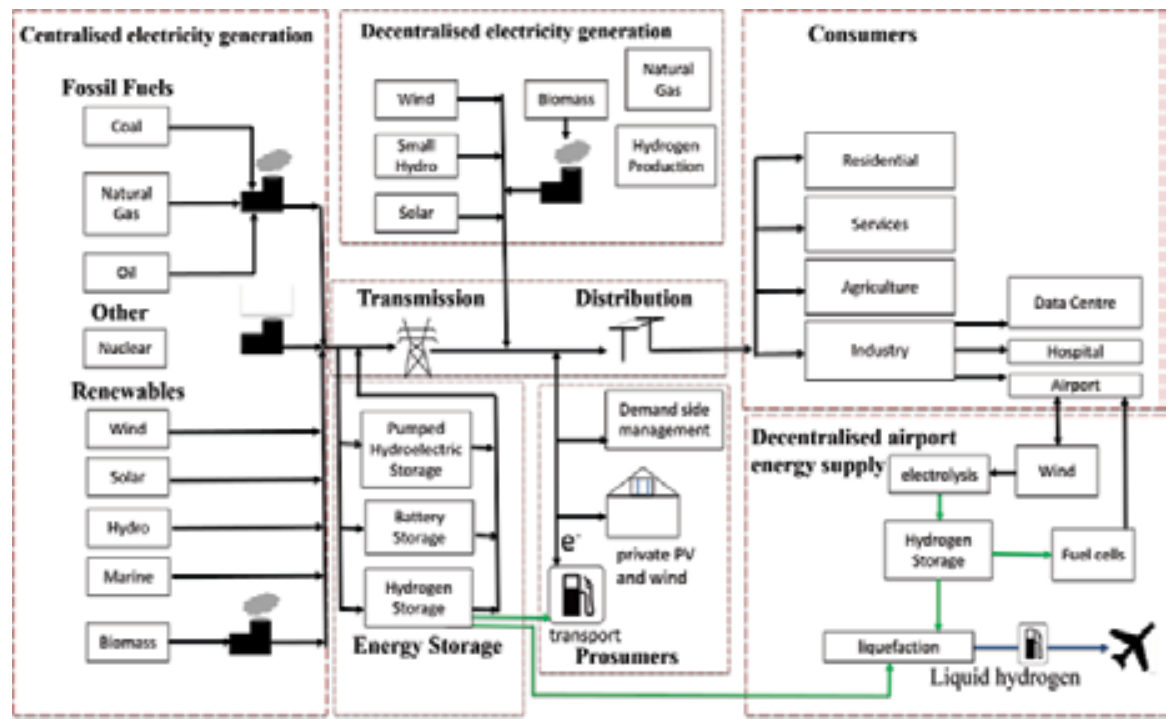

Figure 8. Future integrated hydrogen energy systems utilising added value of liquid hydrogen.

Figure 8 highlights a more complex depiction of Figure 3 with the use of hydrogen not only for storage and electricity generation but also as a fuel for the aviation industry. It should be noted the flexibility of hydrogen as an energy vector being capable of being used for passenger transport, aviation, thermal and cryogenic applications as well as bulk energy storage and electricity generation. With the increasing energy problems and aims to reduce carbon dioxide emissions and energy dependence, the use of hydrogen can now be seen as a viable solution. The support of necessary policy measures can allow the hydrogen economy to emerge in an attempt to mitigate fossil fuel use other energy problems as mentioned.

A centralised hydrogen vision can be considered with the use of low-carbon systems such as nuclear energy and steam methane reforming integrated with carbon capture and storage; however, from Figure 8, a decentralised vision can alternatively be considered as a potential solution. Airbus, a leading aircraft manufacturer, has received a patent for the design of a supersonic passenger plane operated on hydrogen, Figure 9. The plane has three different engine types, and the plane is fuelled by hydrogen and liquid oxygen. The fuel cell is to be held in the cargo hold with the liquid hydrogen tank and heat exchangers located in the tail. The fuel cell in the aircraft transforms chemical energy from the hydrogen into electricity through a chemical reaction with oxygen with waste of water, heat and oxygen-depleted air allowing reduced operation emissions. Such an aircraft can have implications for the aviation sector. Additionally, it is predicted that the water produced can be used to reduce the water required on-board that can reduce the weight and therefore fuel consumption of the aircraft. 


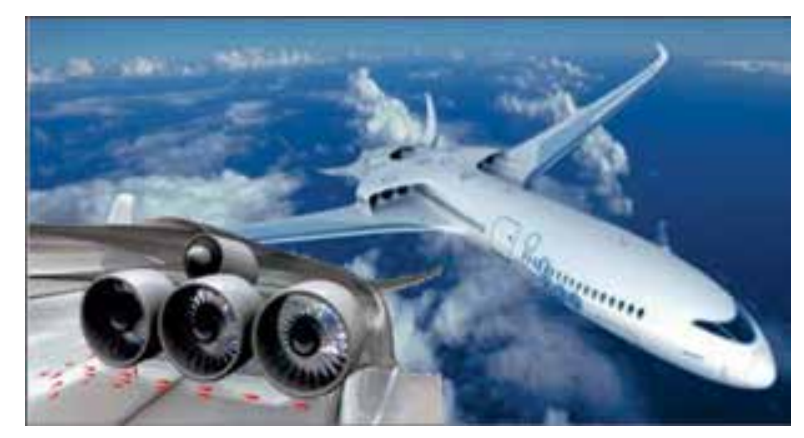

Figure 9. Concept from Airbus for the hydrogen fuelled aircraft [44].

\section{Conclusions}

The results of the investigation into the various energy storage technologies available for energy management of decentralised renewable energy systems highlight the large potential of hydrogen as a storage medium for energy management of decentralised energy systems but also further highlight one concept in which the further value of hydrogen is explored in with regard to an airport scenario. With large focus on the decarbonisation of electricity systems, the need for further opportunities for decarbonisation within the heat and transport sector is required. Decentralised hydrogen energy systems can be a solution to the aircraft industry requirement to lower emissions and reduce dependence on fossil fuels. Hydrogen and other storage technologies can allow a suitable energy carrier for managing the transition to a decarbonised energy system.

\section{Author details}

Bartek A. Glowacki ${ }^{1,2,3^{*}}$ and Emma S Hanley ${ }^{1}$

*Address all correspondence to: bag10@cam.ac.uk

1 Department of Physics and Energy and Bernal Institute, University of Limerick, Castletroy, Limerick, Ireland

2 Department of Materials Science and Metallurgy, University of Cambridge, Cambridge, United Kingdom

3 Institute of Power Engineering, Warsaw, Poland 


\section{References}

[1] US Energy Information Administration. International Energy Statistics. http:// www.eia.gov/cfapps/ipdbproject/iedindex3.cfm?

tid=2\&pid=2\&aid=12\&cid=wW,\&syid=1980\&eyid=2012\&unit=BKWH(accessed 08/12/2015).

[2] US Energy Information Administration. International Energy Statistics. https:// www.eia.gov/cfapps/ipdbproject/iedindex3.cfm?

tid=2\&pid=29\&aid=7\&cid=ww $\&$ syid=2008\&eyid=2012\&unit=MK(accessed 08/12/2015).

[3] Cheung, K.Y., Cheung, S.T., Navin De Silva, R.G., Juvonen, M.P., Singh, R., Woo, J. J.,). Large-Scale Energy Storage Systems. London: Imperial College London (2003).

[4] SBC Energy Institute, 2013, Electricity storage. http://www.sbc.slb.com/SBCInstitute/ Publications/ /media/Files/SBC\%20Energy\%20Institute/SBC\%20Energy\%20Institute_Electricity_Storage\%20Factbook_vf.ashx (accessed 17/09/2015).

[5] Deane J.P., Ó Gallachóir B.P., McKeogh E.J. Techno-economic review of existing and new pumped hydro energy storage plant. Renewable and Sustainable Energy Reviews 2014; 14 (4) 1293-1302.

[6] Eurostat, 2015, Energy from Renewable Sources. http://ec.europa.eu/eurostat/statisticsexplained/index.php/Energy_from_renewable_sources\#Share_of_energy_from_renewable_sources:_heating_and_cooling (accessed 27/01/2016).

[7] European Commission-European Research Area, 2008, Hyways-The European hydrogen roadmap. ftp://ftp.cordis.europa.eu/pub/fp7/energy/docs/hyways-roadmap_en.pdf (accessed 15/09/2015).

[8] Andrews J., Shabani B. Re-envisioning the role of hydrogen in a sustainable energy economy. International Journal of Hydrogen Energy 2012; 37 (20) 1184-1203.

[9] International Energy Agency, 2012, Energy technology perspectives 2012-Pathways to a clean energy system. ISBN: 978-92-64-17488-7.

[10] Saur G., Ainscough C., 2011, U.S. Geographic analysis of the cost of hydrogen from electrolysis. http://www.nrel.gov/hydrogen/pdfs/52640.pdf (accessed 02/04/2015).

[11] Singliar M. Solar energy using for hydrogen production. Petroleum \& Coal 2007; 49 (2) $40-47$.

[12] DOE/NETL-2010/1434, 2010, Assessment of hydrogen production with $\mathrm{CO}_{2}$ capture volume 1: Baseline state of the art plants. http://www.canadiancleanpowercoalition.com/pdf/SMR9\%20-\%20H2_Prod_Vol1_2010.pdf (accessed 18/06/2014). 
[13] Satyapal S., Petrovic J., Read C., Thomas G., Ordaz G. The U.S. Department of energy's national hydrogen storage project: progress towards meeting hydrogen-powered vehicle requirements. Catalysis Today 2007; 120 (3-4) 246-256.

[14] Ross D.K. Hydrogen storage: the major technological barrier to the development of hydrogen fuel cell cars. Vacuum 2006; 80 (10) 1084-1089.

[15] US Department of Energy, 2011, Fuel cells technologies program. http:// www1.eere.energy.gov/hydrogenandfuelcells/pdfs/fct_h2_storage.pdf (accessed 06/07/2015).

[16] Lozano-Castelló, D., Suárez-García,F., Linares-Solano, A., Cazorla-Amorós,D., Chapter 12 - Advances in Hydrogen Storage in Carbon Materials, In Renewable Hydrogen Technologies, edited by Luis M. Gandía, Gurutze Arzamendi and Pedro M. Diéguez, Elsevier, Amsterdam, 2013, pp 269-291, ISBN 9780444563521, http://dx.doi.org/10.1016/ B978-0-444-56352-1.00012-X.

[17] Züttel A. Materials for hydrogen storage. Materials Today 2003; 6 (9) 24-33.

[18] James B.D., 2012, Hydrogen storage cost analysis preliminary results.http:// www.hydrogen.energy.gov/pdfs/review12/st100_james_2012_o.pdf(accessed 06/07/2015).

[19] Johansson T.B., Kelly H., Reddy A.K.N., 1993, Renewable energy: sources for fuels and electricity. http://www.iphe.net/docs/Events/Seville_11-12/Workshop/Presentations/ Session\%203/3.1_IPHE\%20workshop_Harrison.pdf (accessed 02/007/2015).

[20] Weber A.Z., Mench M.M., Meyers J.P., Ross P.N., Gostick J.T., Liu Q. Redox flow batteries: a review. Journal of Applied Electrochemistry 2011; 41, 1137-1164.

[21] Chalamala B.R., Soundappan T., Fisher G.R., Anstey M.R., Viswanathan V.V., Perry M.L. Redox flow batteries: an engineering perspective. Proceedings of the IEEE 2014; 102, 976-999.

[22] Hawthorne K.L., Wainright J.S., Savinell R.F. Studies of iron-ligand complexes for an all-iron flow battery application. Journal of the Electrochemical Society 2014; 161, A1662-A1671.

[23] Petek T.J., Wainright J.S., Savinell R.F. Slurry electrode for an all-iron flow battery for low cost large-scale energy storage. Abstract for 2013 IChE Annual Meeting, Hilton San Francisco, Union Square, San Francisco, CA.

[24] Solarenvi, Cell cube FB 10-100. http://www.solarenvi.cz/assets/Downloads/datasheets/ fv_Cellstrom-Cellcube-FB-10-100.pdf (accessed 10/04/2014).

[25] Hung C.J., Liu C.H., Wang C.H., Chen W.H., Shen C.W., Liang H.C., Ko T.H. Effect of conductive carbon material content and structure in carbon fiber paper made from carbon felt on the performance of a proton exchange membrane fuel cell. Renewable Energy 2015; 78, 364-373. 
[26] Viswanathan V., Crawford A., Stephenson D., Kim S., Wank W., Li B., Coffey G., Thomsen E., Graff G., Balducci P., Kintner-Meyer M., Sprenkle V. Cost and performance model for redox flow batteries. Journal of Power Sources 2014; 247, 1040-1051.

[27] Chakrabarti M.H., Brandon N.P., Hajimolana S.A., Tariq F., Yufit V., Hashim M.A., Hussain M.A., Low C.T., Aravind P.V. Application of carbon materials in redox flow batteries. Journal of Power Sources 2014; 253, 150-166.

[28] Savinell, R.F. Flow batteries a historical perspective. Case Western Reserve University, Department of Chemical Engineering. DOE Flow Batteries Workshop, Lawrence Berkeley National Laboratory (LBNL), 2012.

[29] Hanley E.S., Amarandei G., Glowacki B.A. Potential of redox flow batteries and hydrogen as integrated storage for decentralised energy systems. ACS Energy and Fuels 2016. DOI: 10.1021/acs.energyfuels.5b02805.

[30] Directorate- General for Internal Policies, 2010, Decentralised energy systems. http:// www.europarl.europa.eu/document/activities/cont/ 201106/20110629ATT22897/20110629ATT22897EN.pdf (accessed 15/09/2015).

[31] Winter C.J. Into the hydrogen energy economy-milestones. International Journal of Hydrogen Energy 2005; 30 (7) 681-685.

[32] Marbán G., Valdés-Solís T. Towards the hydrogen economy? International Journal of Hydrogen Energy 2007; 32 (12) 1625-1637.

[33] Rújula A.A.B., Amada J.M., Bernal-Agustín J.L., Yusta Loyo J.M., Navarro J.A.D. Definitions for distributed generation: a revision. http://www.icrepq.com/full-papericrep/295-bayod.pdf (accessed 15/09/2015).

[34] Bouffard F., Kirschen D.S. Centralised and distributed energy systems. Energy Policy 2008, 36 (12) 4504-4508.

[35] El-Khattam W., Salama M.M.A. Distributed generation technologies, definitions and benefits. Electric Power Systems Research 2004; 71 (2) 119-128.

[36] Kaundinya D.P., Balachandra P., Ravindranath N.H. Grid-connected versus standalone energy systems for decentralized power-A review of literature. Renewable and sustainable energy reviews 2009; 13 (8) 2041-2050.

[37] Glowacki B.A., Nuttall W.J., Clarke R.H., 2013. Beyond the helium conundrum. http:// ieeexplore.ieee.org/xpls/abs_all.jsp?arnumber=6425422 (accessed 15/06/2014).

[38] Nuttall W.J., Clarke R., Glowacki B.A. The future of helium as a natural Resource, Routledge. Taylor \& Francis Group Ltd, Oxford 2012. ISBN: 978-0-415-57697-0.

[39] Glowacki B.A., Majoros M., Vickers M., Evetts J.E., Shi Y., McDougall I. Superconductivity of powder-in-tube $\mathrm{MgB}_{2}$ wires Superconductor Science and Technology 2001; 14 (4) 193. 
[40] Patel A., Hopkins S.C., Giunchi G., Figini AA., Shi Y., Palka R., Cardwell D., Glowacki B.A. The use of an $\mathrm{MgB}_{2}$ hollow cylinder and pulse magnetized for magnetic levitation applications. IEEE Transactions Applied Superconductivity 2013; 23 (3).DOI: 10.1109/ TASC.2012.2236143.

[41] Sander M., Brighenti F., Gehring R., Jordan T., Klaeser M., Kraft D., Mueller R., Neumann H., Schneider T., Stern G. LIQHYSMES-Liquid $\mathrm{H}_{2}$ and SMES for renewable energy applications. International Journal of Hydrogen Energy 2014; 39 (23) 1200712017.

[42] Sander M., Gehring R., Neumann H., Jordan T. LIQHYSMES storage unit-Hybrid energy storage concept combining liquefied hydrogen with superconducting magnetic energy storage. International Journal of Hydrogen Energy 2012; 37 (19) 14300-14306.

[43] Makida Y., Shintomi T., Asami T., Suzuki G., Takao T., Hamajima T., Tsuda M., Miyagi D., Munakata K., Kajiwara M. Design study of the cooling scheme for SMES system in ASPCS by using liquid hydrogen. Physica C: Superconductivity 2013; 494 (15) 208-212.

[44] Airbus. Fuel Cells. http://www.airbus.com/innovation/future-by-airbus/future-energy -sources/fuel-cells/ (accessed 30/01/2016).

[45] Cheadle M.J., Wozniak M., Bromberg L., Glowacki B.A., Jiang X., Zeng R., Minervini J.V., Brisson J.G. DC Superconducting Cable Using $\mathrm{MgB}_{2}$ Wires IEEE Transactions on Applied Superconductivity 2013; 23.DOI: 10.1109/TASC.2013.2248313.

[46] Chen J.Y.C. Spin Isomers of Molecular Hydrogen and Improved Sensitivity for NMR and MRI. https://www.organicdivision.org/ama/orig/Fellowship/2009_2010_Awardees/Essays/Chen.pdf (accessed 28/02/2016).

[47] MacDonald K.T., Iarocci M., Kirk H.G., Mulholland, G.T., Titus P.H., Weggel R.J. Use of He gas cooled by liquid hydrogen with a 15-T pulsed copper solenoid magnet. Technical/Economic Report August 15 2002; 1-5. 


\section{Section 4}

Energy Management Systems for Smart Homes 

Chapter 9

\title{
Securing the Home Energy Management Platform
}

\author{
Søren Aagaard Mikkelsen and \\ Rune Hylsberg Jacobsen \\ Additional information is available at the end of the chapter \\ http://dx.doi.org/10.5772/62923
}

\begin{abstract}
Energy management in households gets increasingly more attention in the struggle to integrate more sustainable energy sources. Especially in the electrical system, smart grid systems are envisioned to be part in the efforts towards a better utilisation of the energy production and distribution infrastructure. The Home Energy Management System (HEMS) is a critical infrastructure component in this endeavour. Its main goal is to enable energy services utilising smart devices in the households based on the interest of the residential consumers and external actors. With the role of being both an essential link in the communication infrastructure for balancing the electrical grid and a surveillance unit in private homes, security and privacy become essential to address. In this chapter, we identify and address potential threats Home Energy Management Platform (HEMP) developers should consider in the progress of designing architecture, selecting hardware and building software. Our approach starts with a general view of the involved stakeholders and the HEMS. Given the system overview, a threat model is constructed from the HEMP developer's point of view. Based on the threats that have been detected, possible mitigation strategies are proposed taking into account the state of the art of technology for securing platforms.
\end{abstract}

Keywords: smart grid, IoT, security, privacy, cloud, web service, service-oriented architecture

\section{Introduction}

A smart grid is a vision of a more intelligent electrical infrastructure. It is envisioned to provide a more reliable and effective electrical grid in the process of integrating more intermittent renewable energy sources, like wind power and solar power. This will demand for systems that 
will continuously monitor and manage end-points of the grid, for example, an end-point as the residential sector that consumes around $31 \%$ of the electricity worldwide [1] $]^{1}$ These systems are known as Energy Management Systems (EMSs). EMSs aim at utilising the grid capacity more efficiently in terms of instantaneous demand and generation. This includes flattening the peak demand by giving the demand-side awareness about the time periods it is "smart" to consume energy [2].

Ensuring security and privacy are becoming important issues to address with the deployment of smart meters. Smart meters are devices that record the electric consumption and production and communicates this information automatically to the utility. Research has shown that information from smart meters can reveal personal habits and disclose details about the residents living there. Furthermore, the situation exacerbates if additional meters and actuators are installed on the most consuming devices for Direct Load Control (DLC) in the demand response paradigm. Therefore, security and privacy enhanced system architectures suggest, a Home Energy Management System (HEMS) that runs locally inside the residential home, among other things to resolve privacy issues.

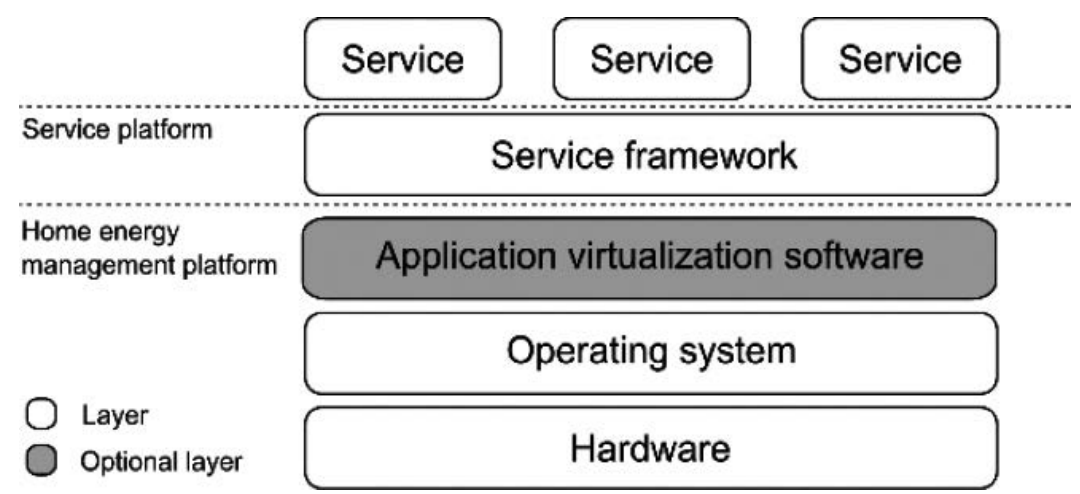

Figure 1. The considered stack for a HEMS.

This chapter describes security and privacy issues for a HEMS in an SOA. It focuses on the Home Energy Management Platform (HEMP) provider [also in other contexts referred to as the Original Equipment Manufacturer (OEM)]. A HEMP provider is responsible for the platform, which the software developers and service providers can deploy their software and services on as seen in Figure 1. A HEMP provider creates the platform that facilitates security and privacy in several layers. Following a holistic approach, a threat modelling approach is used that considers the interdependencies between stakeholders of the system before identifying the threats the system faces. A risk assessment is made on the identified threats, and

\footnotetext{
${ }^{1}$ Percentage based on the average electricity consumption from residential domains in Spain, Norway and Denmark. ${ }^{2}$ EMSs can refer to not only systems that support the operation of the electrical grid on a transmission and distribution level but also systems that automatically control and monitor energy usage in buildings.
} 
possible mitigation strategies are presented based on a review of the state of the art in hardware security. Based on the threat analysis, recommendations to create a secure HEMS are provided.

\section{Background and related work}

A preliminary review of the domain is presented before developing a threat model for the HEMP. The review includes a description of general threat modelling approaches together with commonly used diagrams to perform the analysis.

\subsection{Threat modelling approaches}

Threat modelling is a systematic approach that supports the process of finding the appropriate technical solutions for enforcing security and privacy. It considers the potential threats with the greatest impact and addresses these first.

There are generally three approaches to threat modelling [3]:

- Assets-centric: Assets define things that need protection, or things the attacker wants or something in between those two. The approach focusses on defining the assets of the system and afterwards, analysing the flow of assets within an organisation. The model can then be applied with an attack set like STRIDE [3], which is a mnemonic for Spoofing, Tampering, Repudiation, Information disclosure, Denial of service and Elevation of Privilege.

- Attacker-centric: Envisioned attackers are defined in terms of resources and capabilities. An attacker model identifies a number of scenarios where an attacker can break the system both directly and indirectly. Directly, in terms of exploiting, for example, software vulnerabilities, and indirectly, in terms of exploiting humans that have privileges within the system.

- System-centric ${ }^{3}$ : The approach attempts to look at the designed system as a whole. As the system is being designed, the threat model is continuously being developed or updated. The threat model includes finding threats using, for example, STRIDE and attack trees [3].

When comparing the approaches, the system-centric approach more tightly couples with the development process, whereas the asset-centric approach and attacker-centric approach can be considered more "detached" and are typically performed separate from the software development.

\subsubsection{Diagrams for threat modelling}

The typical diagrams for revealing threats focus on describing the domain or the data flows within the considered system. The goals of these diagrams are to create a common view of the system and to make the communication paths visible. These diagrams provide an abstraction

\footnotetext{
${ }^{3}$ Is often called software-centric, but for the sake of generality we use the term system-centric.
} 
of the system which allows system architects and software developers to create a common view of the system and define the system boundaries.

The Unified Modelling Language (UML) [4] is general-purpose modelling language that specifies a set of diagrams for modelling systems. It provides a standardised way of modelling systems and provides two fundamental representations of a system: behavioural models and structural models. Behavioural models, such as use case diagrams, can be beneficial to identify stakeholders and describe a proposed functionality. Structural models, such as class diagrams, are useful for describing the domain which includes objects, attributions, operations and relationships.

Data Flow Diagrams (DFDs) have traditionally been used for threat modelling, since the threat problems tend to follow the data flow not the control flow [3]. It is used for describing how data enter and leave the system. The basic elements for modelling the system are shown in Table 1. The system is typically modelled with specific scope, where the external system that provides the system with data is modelled using the external entity element. The system of interest consists of a number of processes and data stores with data flow between them. To define the boundary between trust elements and non-trusted elements, DFD also includes trust boundaries. Trust boundaries visualise where data flows intersect between the system and a malicious actor.

\begin{tabular}{llll}
\hline Element & Model & Description & Examples \\
\hline External entity & $\begin{array}{l}\text { Outside scope } \\
\text { entity }\end{array}$ & $\begin{array}{l}\text { A stakeholder delivering or receiving } \\
\text { to the system }\end{array}$ \\
Process & Execution of system element & Executable code or hardware function \\
Data flow & Communication between & RPC calls, system calls, HTTP \\
Data store & elements & Files, memory, database \\
\hline
\end{tabular}

Table 1. The basic elements of a DFD.

By modelling the specific system with DFD elements, it is possible to map these elements to the STRIDE threats. For instance, an external entity for the system can generate a spoofing threat, that is, an external entity can pretend to be something or someone else than originally thought 
of. By enforcing an authentication mechanism, it is possible to mitigate the threat if the external entity was trying to spoof a person or a thing.

\subsection{Home energy management software platforms}

As depicted in Figure 1, the HEMS consists of a software platform that provides the middleware for interoperable communication between devices and services. In the following, two open source EMSs are presented. Both use the OSGi (Open Service Gateway Initiative) architecture that specifies a modular and service-based software platform implemented in Java.

\subsubsection{OpenHAB}

The openHAB project ${ }^{4}$ is an open software framework that focusses on enabling home automation by joining systems and technologies available in the smart home domain. It allows for automation rules and offers a single user interface for all such systems. The openHAB software is capable of running on any device that supports the Java Virtual Machine (JVM). The architecture consists of three main components: the OSGi framework, openHAB Core Components and openHAB Add-ons. The OSGi framework and the openHAB Core Components represent the internal communication infrastructure and base libraries. The openHAB Add-ons include a large set of protocol bindings that map between other home automation protocols to an abstract data model. It includes an "item" repository, where an item can be interpreted as an abstraction for a property a device can actuate. The openHAB framework provides mechanisms for securing the communication to the openHAB software platform, but does not have additional security features beside what the OSGi framework can provide.

\subsubsection{OGEMA}

Open Gateway Energy Management (OGEMA) is an open software framework for smart grid services. It represents a system that supports building automation control and energy management for residential and industrial environments. The framework rests on a hardwareindependent platform with a common execution environment for all deployable services. OGEMA uses the OSGi Java framework enabling a modular and dynamic software environment. The OGEMA framework consists of the following entities:

- OGEMA Services: Common services like resource administration, user interface, web interface and data logging, but also a common data model and access control.

- Resources: Data structures according to the data model representing the connected devices.

- Applications: Services for, for example, price-based management and energy analysis.

- Communication drivers: Drivers for supporting multiple communication media, for example, ZigBee and EEBus.

\footnotetext{
${ }^{4}$ http://www.openhab.org/

${ }^{5} \mathrm{http}$ //www.ogema.org/
} 
The OGEMA architecture embeds three "modes" of security: standard infrastructure, a controlled environment and proof of security [5]. Furthermore, it isolates third-party applications which can be integrity checked using the public key of the service provider. This is similar to Android's permission handling [5]. Since OGEMA framework is restricted to security capability of the OSGi Java framework and the JVM, it is possible for a malicious component to freeze the platform by allocating too much memory or changing shared variables. A possible solution to this issue is the I-JVM presented in [6].

\subsection{Threat analysis of the smart grid domain}

Cyber-Physical Systems (CPSs) and Internet of Things (IoTs) are two research domains that overlap and share similar challenges with the smart grid domain. In [7], they identify four key challenges in designing a secure IoT which include data management, identity management, trust management and privacy. Based on these challenges, they propose hardware-based mitigation strategies that include Physical Unclonable Function (PUF) for data provenance, integrity and identity management. However, they present a non-systematic approach where the stakeholders are not clearly identified. In reference [8], they consider threat modelling issues of CPSs. The authors provide a generic model of a CPS and present a case study with a road vehicle. In reference [9], researchers present a survey of security theories, analysis, simulation and application fields but without giving recommendations.

In reference [10], they present a structured method for identifying security threats in smart home scenarios. It is based on a context pattern for the elicitation of domain knowledge for the smart home domain. Using the context pattern for creating DFDs for the smart domain, they identify the entry points and vulnerabilities. This allows them to define the attack paths from entry point to the assets of the system. Their method is sound and structured but lacks possible threat mitigation strategies.

\section{Threat modelling of a home energy management platform}

Security experts are encouraging designers to make explicit statements about the security assumptions, when designing and implementing systems. Defining the security assumptions is the first step in order to find the vulnerabilities of a system. With a description of the vulnerabilities, it is possible to assess the risks a given design exposes. Security technologies can then be enforced where the vulnerabilities need to be addressed.

\subsection{Methodology}

A systematic approach is necessary to discover all the vulnerabilities of a system [10]. Our approach for designing a HEMP is shown in Figure 2, consisting of five steps. The methodology is based on the work presented in references [3,10,11], but adapted to our work. 


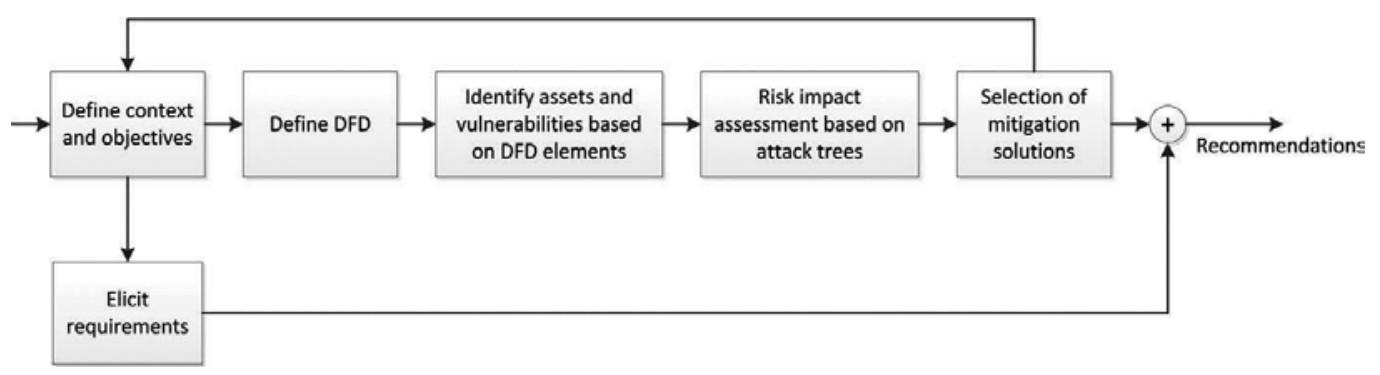

Figure 2. The methodology for performing the threat modelling.

The following gives a description of the six steps in our method:

Step 1. Define context and objectives-The context represents the identification of the main stakeholders of the system, the general system architecture and the desired objectives. The purpose of this step is to create the scope of interest and focus on the analysis. Part of the context is the review of regulations with the smart grid area and to describe the considered use cases. This includes also defining the dependency between stakeholders. With the definition of the context and objectives, requirements can be elicited.

Step 2. Define DFD-Using the previously defined context, the flow of information is modelled through DFDs. Context elements are mapped to elements that present input/output, processes, data flow and data storage. In the modelling process, the context can be refined, for example, adding additional stakeholders or modifying the system architecture. Furthermore, it supports the process of modelling the domain knowledge to DFD elements. With the process of defining the context and modelling, the DFD represent the high-level system description.

Step 3. Identify assets and vulnerabilitiesbased on DFD elements-Based on the DFD elements representing the HEMP, the system's assets and threats are identified. Assets represent valuable targets for an attacker and are therefore of interest for the attacker. Assets can be both physical and linked to a process. One approach to identify threats for the identified assets is the STRIDE-per-element or STRIDE-per-interaction approach [3].

Step 4. Risk impact assessment based on attack trees-With explicit threats, the risk impact assessment can be performed using attack trees. The step assesses how noticeable and with what likelihood a threat is. The outcome of this assessment can be either accepted or mitigated using a technical solution. An accepted risk represents a possible attack, but it is typically regarded as unlikely.

Step 5. Selection of mitigation approach - If a threat is unacceptable, a selection of a mitigation solution must be considered. The solution can be based on possible software or hardware technologies that consider an attacker with the same power or more. Mitigation can require a different hardware or software solution. The risk assessment of the threat can help in determining the threat mitigation should be placed in the hardware or software. 


\subsection{Stakeholders, system architecture and objectives}

In Figure 3, the major stakeholders of the HEMS are presented. The residential consumer enables the deployment of HEMSs. Trust to the system, reduction of/control of electricity bills, addressing environmental concerns and better comfort (i.e., provision of technical solutions for better control of own energy use) are the main motivational factors for the residential consumers to adopt a smart grid solution [12]. Smart device vendors build sensors and actuators deployed in homes, for example, Wink, ${ }^{6}$ SmartThings ${ }^{7}$ or Vera ${ }^{8}$ represent an envisioned smart device vendor. Often these vendors can be categorised whether or not they provide a total home automation solution or have upgraded an existing product to be IoTenabled. In the envisioned system, the Distribution System Operator (grid operator) is not directly interacting with the HEMS, instead it depends on the smart grid services developed by service providers and deployed by the service market responsible. Services utilise information retrieved from the residential homes and electric grid, to improve electricity usage for both the residential consumers and grid operator. For connecting the services and the smart devices, a communication service provider (CSP) is needed. The software platform providers create the software platform, where services can be executed.

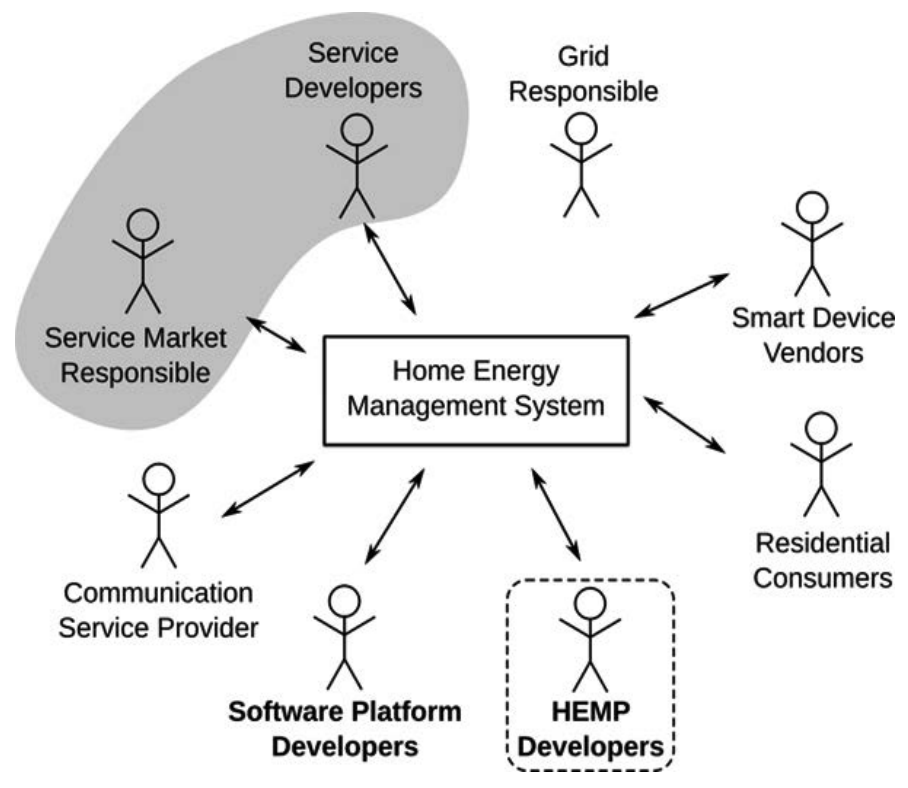

Figure 3. Overview of the major stakeholders of the HEMS. It is inspired by [32].

The HEMS is considered to be placed in an SOA, where there exist home-oriented and gridoriented services. The services are implemented as web services based on the REpresentational

\footnotetext{
${ }^{6}$ http://www.wink.com/

${ }^{7}$ https://www.smartthings.com/

${ }^{8} \mathrm{http}: / /$ getvera.com/
} 
State Transfer (REST) architectural style. The system considers many-to-one mapping between the residential consumer and the DSO, that is, the home-oriented services are executed for each residential consumer, whereas the grid-oriented services utilise the output for optimising the operation for the DSO.

The rationale of the system architecture is that the home-oriented services will optimise according to demands of individual homes, whereas the grid-oriented services will support the operation of the electrical grid. Since grid-oriented services can be highly valuable for the grid responsible (DSO), these will foster the development of the home-oriented services. A fundamental requirement for the approach is that the services can be used as building blocks for delivering additional services. For a more detailed insight into the system, the reader is referred to references $[13,14]$.

The main characteristics of a service-oriented system are [15]:

- Heterogeneous platform and execution environment.

- Communication is handled through standards.

- Advances the concept of component-based software by reducing the coupling between services.

- Encourage continuous and independent (re)deployment of software.

The domain model is depicted in Figure 4 using a UML class diagram together with the main stakeholders. It is composed of classes (rectangular icons), packages (folder icons) and actors (stick figures). It considers the domain to consist of two major parts: a service market and a residential home. Both parts include a number of elements (represented as classes) that represent the implementation by a stakeholder. At this stage, only elements that are relevant for an identified stakeholder are considered. The relationship between the elements indicates the

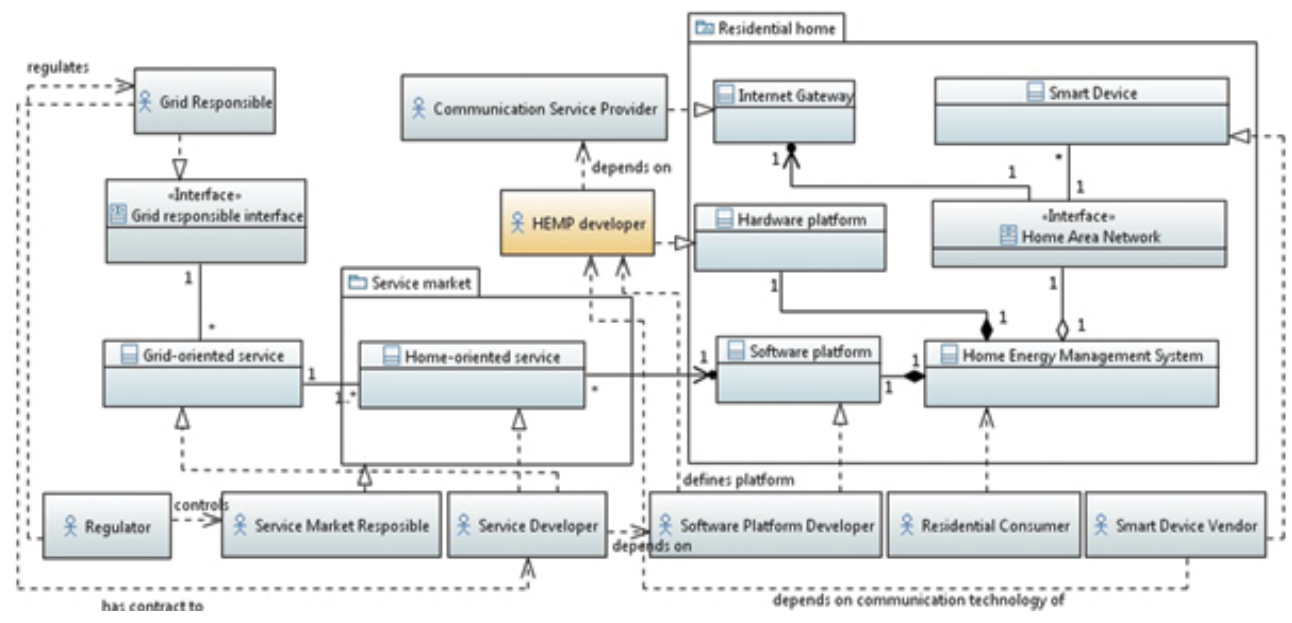

Figure 4. Domain model of the system. 
multiplicity and type of association. Besides the basic UML arrows representing the associations, two other arrows are drawn. The dependency between the stakeholders is modelled with an open arrow to visualise how stakeholder's business objectives are associated with other stakeholders. A closed arrow indicates the realisation of the element.

In the process of defining hardware recommendations for the HEMP developer, focus is set on the HEMP developer as depicted and highlighted in Figure 3. As seen in Figure 4, the HEMP developer has several dependencies - either directly or indirectly.

To this end, the objective of the HEMP developer for the HEMS is the following:

The HEMP will provide a security and privacy enhanced platform for service developers to deploy on web service on, while being a trustworthy platform for the residential consumers, service providers and the grid responsible.

\subsection{Requirements}

To identify the security threats and vulnerabilities of the HEMP (see Section 3.5), the necessary requirements, which the platform must adhere to, have to be explicitly stated. An explicit set of requirements can guide the process of designing a platform which is resistant to possible attacks. Furthermore, it allows for a risk assessment of third-party services installed on the device.

Requirements are usually understood as stating what a system is supposed to do-contrary to how it should do it [16]. It characterises the desired functional behaviour and performance, whereas non-functional requirements aim at fulfilling a desired property. Non-functional requirements are usually judged by the operator of the system and relate more to the system architecture, whereas functional requirements are testable.

In the following, both the functional and non-functional requirements for the HEMP will be presented. The requirements are classified according to the direct stakeholders for the HEMP developer. The rationale is that the requirements and dependencies from the stakeholders will have an impact on the threats and thereby on the mitigation strategies that technical solutions can provide. The listed requirements are derived from use cases and requirements specified in references [14, 17-19].

\subsubsection{Software platform developer}

An essential feature of the HEMS is to provide an environment for executing services relevant for external actors in the smart grid. This includes services with real-time dependencies or not. These services can be manufactured by different service providers and therefore depend on an isolated execution environment, if a single service should not crash the system. Moreover, software trends dictate a continuous development and maintenance process to follow an agile development life cycle and fix newly discovered vulnerabilities. Thus, doing updates to the services is vital for its sustainability. Finally, a software platform is responsible for delivering the "knobs" of the smart devices for services and external system to interact with in a secure way. The requirements are as follows: 
R1. The HEMS shall support execution of real-time dependent services, like services for controlling a residential battery according to external signals.

R2. The HEMS employ separation mechanisms to securely isolate services.

R3. The HEMS shall be able to upgrade existing services.

R4. The HEMS shall support remote software updates from the software framework developers for managing services.

R5. The HEMS shall provide security measures appropriate for the protection of integrity and confidentiality of services.

These requirements relate to service providers which represent the main stakeholder of the software platform developer.

\subsubsection{Smart device vendor}

End devices in the smart grids often represent the load of the electrical system. Giving these devices the ability to communicate with other devices facilitates information to be disseminated to all stakeholders. For the smart devices to be part of the smart grid, a communication infrastructure in the Home Area Network (HAN) that incorporates a heterogeneous set of communication protocols is essential [20,21]. Furthermore, for smart devices to provide a secure communication path, it is necessary to enable end-to-end encryption. The smart device vendors' requirements are as follows:

R1. The HEMS shall support multiple communication protocols for the HAN.

R2. The HEMS shall provide smart device vendors with the ability to extend the set of communication drivers easily.

R3. The HEMS shall facilitate end-to-end encryption between smart devices and the services that utilise and control the smart devices.

Besides depending on the physical communication technology implemented on the HEMS, software drivers are also necessary to provide an interface for a service.

\subsubsection{Residential consumer}

The intelligent automation of the smart grid depends on the residential consumers. They will be an integral part that will ensure reliability of the electrical grid by modifying the way energy is used. A HEMS can support the behavioural change but requires the acceptance of the residential consumer. It is generally believed that a user-centric approach is vital, where trust to the system remains one of the priorities [12]. The requirements for a residential consumer are:

R1. The HEMS must secure the confidentially and integrity of meter data.

R2. The HEMS should provide residential consumers the ability to install and uninstall services on demand. 


\subsection{Data flow diagram of the home energy management platform}

The main purpose of DFDs is to identify the key data flows in the system. Modelling the data flows, contrary to the control flows, reveals how information is exchanged throughout the system. A DFD model explains who takes part in a process for delivering the information, the information needed to complete a process and what information is stored.

Figure 5 provides an overview of the internal data flows on a HEMS and the HEMS interactions with the external entities based on reference [14]. Besides the DFD symbols presented in Table 1, the DFD is annotated with dotted lines indicating the trust boundaries. These are quantified three trust levels from the HEMP provider's point of view. Furthermore, it contains dotted arrows between processes, to indicate how a process affects another process's runtime. These are useful for expressing hardware-related issues.

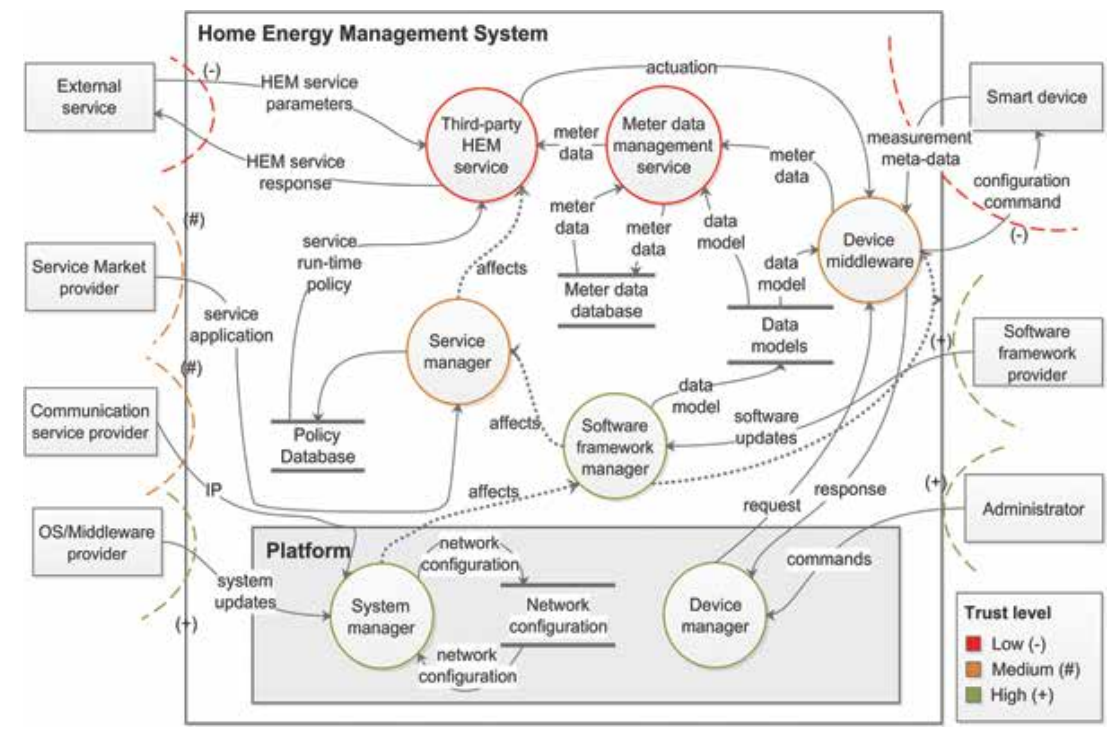

Figure 5. DFD of the HEMS.

As seen in Figure 5, seven processes are included in the model. This includes two services: third-party HEM service and a Meter Data Management (MDM) service. The MDM service is explicitly included to show the data flow of meter data and storage of the smart device configurations. The third-party HEM service presents any high-level service useful for smart grid operations. The service manager and software framework manager relate to the continuous maintenance of the software on the HEMS. Lastly, there is a system manager and device manager for the physical interaction with the HEMS.

\subsection{Threat analysis}

A threat model is a depiction of a system's attack surface. An attack surface has numerous threats towards a set of assets within the system. The purpose of mitigation techniques is to 
protect the assets from a possible attacker. The envisioned attacker can be assigned capabilities, such as being able to have physical access to the system or only have access through the network. Other attackers include social engineers which through "social" interaction can gain unauthorised privileges to the system. This could be attackers using phishing emails to exploit vulnerable code in installed software.

The threats for the HEMP provider are a combination of the indirect threats that the software platform provider faces and the external entities that have physical access to the HEMS. In the following, we classify the considered attacker, identify the assets of the HEMS and elicit a subset of vulnerabilities.

\subsubsection{Assets of the HEMS}

Assets represent valuable targets for an attacker, and therefore naturally represent the system susceptibility for threats. In the following, the key assets are identified with a description of the reason for its inclusion:

Services: These represent both value-added services for the residential consumer, but also account for the foundation of service market generation. A compromised service can lead to cyber attacks on homely property. Furthermore, it can also lead to violation of the intellectual property for the service provider, thus undermining a service market. The services are dependent on the software platform provider to secure the services, where it assumes the underlying software stack (e.g., virtual machine, Operating System (OS), etc.) will not be compromised. The services can contain a range of sensible information regarding the residential consumer, for example, granular meter data, personal identifiable information, cryptographic key sets and so on.

Service manager: This process is responsible for presenting, installing, updating and uninstalling services on the HEMS. It is in direct contact with the residential consumer and service market provider. An attack on the service market manager can lead to undermining the service ecosystem with services unable to be update if a critical software patch needs to be installed. It depends on the given privileges from the software platform. It contains the audit of installed services and cryptographic keys to allow for a secure connection to the smart market provider.

System manager: This manager administrates the OS stack including middleware software (JVM, Python interpreter), system libraries and kernel (network interface, memory management, I/O interface, etc.), and therefore performs privileged operations. The entire software framework stack depends on its implementation. Therefore, it is assumed that it is configured and maintained reliably.

\subsubsection{Identifying vulnerabilities}

Vulnerabilities represent an attacker's entry points to the system. It is therefore crucial to identify these entry points in order to mitigate possible attacks. Here the vulnerabilities are presented using the STRIDE-per-interaction approach between the service and service manager. Other interactions identified in the DFD are omitted for brevity. 


\subsubsection{Services/service manager}

T1. Spoofing: The HEMS contacts the service market provider for exploring, installing, updating the services on Smart Market provider. If the attacker is able to insert spoofed IP packets or DNS packets when the service manager sends requests to the original Service Market site, the service manager can be sent to a site with malicious services.

T2. Tampering: An attacker can inject code for replacing integrity check of installed services. This will lead to malicious services being installed, which were not verified by the service market controller.

T3. Repudiation: Someone rejects installation of a service in order to deny possible payment for energy bill.

T4. Information disclosure: An attacker is able to see the intellectual property of a service.

T5. Denial of service: If an attacker can block any communication with the service manager, the service manager cannot update installed services.

T6. Elevation of privileges: The HEMS supports third-party services to be installed through the service market provider. If the service market provider is able to install a service that is able to access outside the boundary of its privileges, an attacker can access the meter data, smart device configurations.

\subsubsection{Attack trees}

Attack trees are useful for visualising the escalation of attacks (see Figure 6). The attack tree shows the root cause of an attack and what an attack subsequently after would allow an attacker to do. What would seem to be a minor attack on the system can propagate through the system, leaving assets exposed for an attacker.

In the following, the threats towards the process with the lowest trust boundary is focussed on for brevity; the "Third-party HEM service" (where the MDM service is a special case). The threats T1, T2, T4, T5 and T6 can be collected from the root threat "Install of malicious service". The root of the attack tree is set to this threat. It is presented with the problematic state as root. Each edge in the attack tree is labelled with "\{noticeable/likelihood\}" measure, to indicate if the attack is believed to be noticeable and the likelihood of such an approach (Figure 6).

\section{Mitigation strategies}

Often the time spend on judging the level of the risk should be compared to the time for addressing the threat. When addressing the threat, it is possible to mitigate the risk by redesigning of system architecture, implementing a mitigation feature or simply ignoring it. In the following, the chapter will review for hardware security and privacy mechanisms that mitigate the "install of malicious service" attack.

\footnotetext{
${ }^{9}$ The term "someone" instead of attacker is used deliberately, since it can represent the user of the system as well.
} 


\subsection{Security and privacy technologies}

The process of developing a secure hardware platform has recently intensified. Previously, computer security primarily focussed on creating secure software architectures and protocols with the assumption that the lower-layer applications (e.g., the OS) would be secure. However, flaws in an OS implementation can likely lead to additional vulnerability of the application on top of it. Therefore, researchers have proposed systems for running trusted code on an untrusted OS, but there exists still pitfalls, for example, Iago attacks [22]. In the following, the concept of a Trusted Execution Environment (TEE) will be reviewed as well as technologies that use this concept.

\subsubsection{Trusted execution environments}

In its essence, a TEE is an environment that you can choose to rely upon to perform sensitive tasks. The goal of TEE is to provide an isolated execution environment, secure storage, remote attestation, secure provisioning and a trusted path [23]. In hardware, it usually defines a distinguished part of the hardware architecture which is encrypted and integrity protected. It isolates an area of the processor, memory and peripherals for performing privileged operations. Next to the TEE, a Rich Execution Environment (REE) is considered outside the Trusted Computing Base (TCB), where both an untrusted OS and untrusted third-party services can be executed. Despite its realisation in AMD Secure Execution Environment, ARM's TrustZone technology [24], and Intel's SafeGuard Extensions (SGX) [25], it is not widely used by service

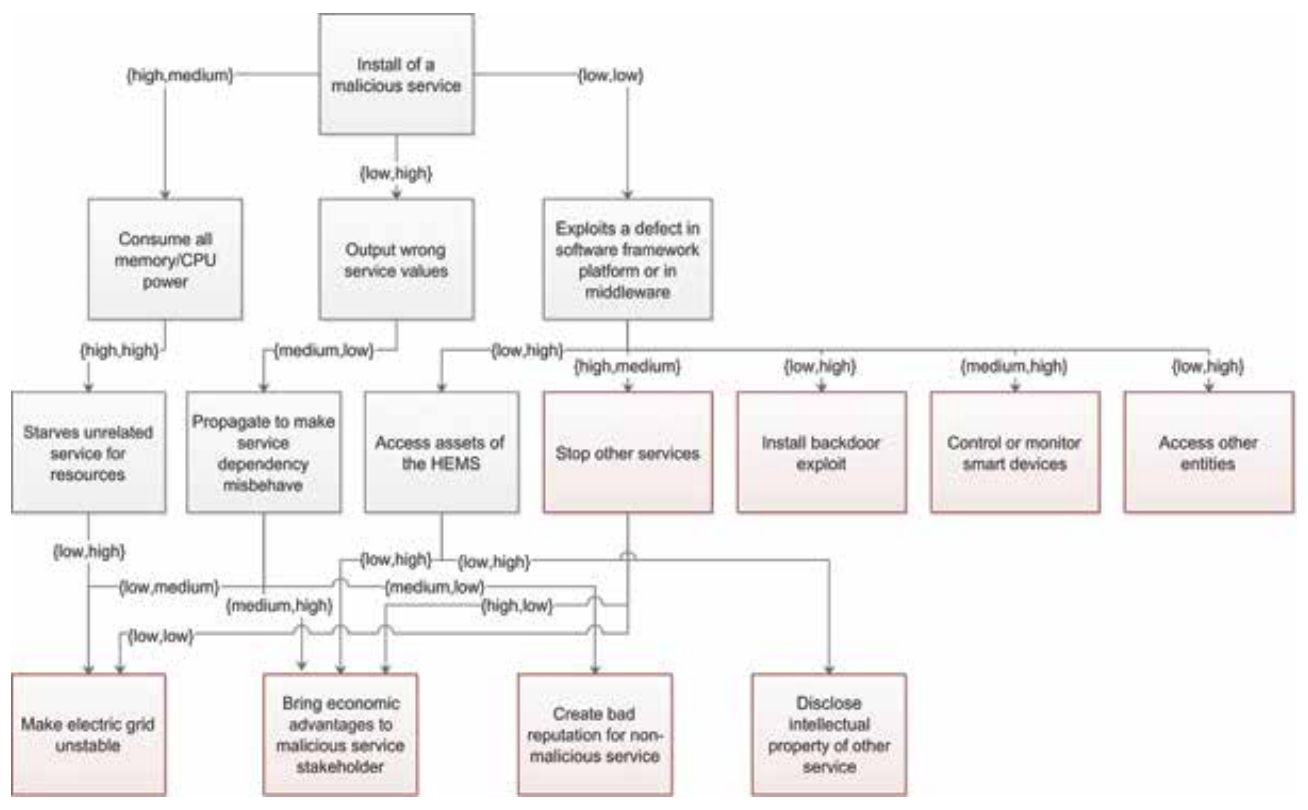

Figure 6. Attacks based on threats towards "Third-party HEM service". 
developers. In the following, we will examine the hardware security technologies for mitigating the identified risk in Section 3.4.

\subsubsection{ARM TrustZone technology}

The security extensions embedded in the specification of ARMv6 and later are called the TrustZone technology [24]. It provides two operational worlds: a normal world and secure world. This allows for different execution privileges for applications. For instance, an application responsible for handling confidential data can be executed in the secure world, without a normal application being aware about; even with vulnerabilities in the normal world's OS. The two worlds are executed through two virtual processors with hardware access controls to switch between the two worlds. The hardware access switch defines the active components in the hardware when switched. Traditionally, ARM TrustZone has primarily been used for Digital Rights Management (DRM) or banking applications, but are not restricted to those types of applications.

\subsubsection{Intel SGX}

Intel's SGX extension is a set of instructions and mechanisms for managing the memory access to the Intel Architecture processors [25]. The main principle relies upon the concept of a protected memory container, also referred to as an enclave. The enclave can be created through application code, where sensitive data are explicitly marked. When the application is executed, a sensitive part of the application's memory space is encapsulated within an enclave. This enclave ensures that the confidentially and integrity of that memory space are sustained even with the presence of privileged malware (i.e., super-user capabilities). Furthermore, to ensure the integrity of the application inside the enclave, Intel SGX supports CPU-based attestation and sealing. An audited process monitors the built process of the enclave and assesses the identity of the hardware from where the enclave should be executed. Before the application is executed of the CPU, the identity of the hardware is verified through the sealing identity. It contains a digital signature (known as report) of the enclave's initial state. The enclave is then capable of receiving a report of the state, verifying its correctness. To verify that the correct software has been instantiated on the platform to remote system, it can perform an attestation with the SGX-supported processor (known as quote). This can prove to a remote system that application has been sent to a genuine SGX implementation. The reader is referred to [26] for a thorough description of the Intel SGX technology.

\subsubsection{TrustLite and TyTAN}

TrustLite [27] is a security architecture for resource constrained embedded systems that generally have to be cost effective in terms of development and production costs. The architecture allows for software isolation with execution-aware memory protection. The memory protection enforces a strict access control on memory by considering the program counter. Furthermore, it includes a secure exception engine that protects tasks from unauthorised exception handling. It has a secure loader that enables update of the security policy as well as prevents memory leakages after resetting the platform. However, TrustLite is static in terms 
of loading software components and their isolation, since this is required at boot time. TyTAN [28] leverages the TrustLite architecture for providing dynamically loading software components together with an integrated real-time system.

\subsubsection{Haven}

The objective of Haven security architecture [29] is to protect the confidentiality and integrity of a user's unmodified application in the cloud from an untrusted cloud provider. It is assumed that the processor is implemented correctly, but otherwise it is assumed that the adversary can access everything else, including memory and I/O devices. On software level, it is assumed that the adversary has full access to the entire software stack, including the OS, hypervisor, Basic Input/Output System (BIOS), device firmware and so on.

The inventors of Haven solve the confidentiality and integrity problem by using an inverse sandboxing technique also known as a shielded execution. Their solution is called Haven, and it leverages the Intel SGX and Microsoft's Drawbridge project [30]. Intel SGX allows application developers to protect their data from unauthorised access or modification by software that have highest privilege levels (e.g., a super-user).

The deployment process of the Haven application is similar to the deployment of a regular cloud application, with the additional step of verifying that the process was correctly performed (the attestation from the Intel CPU). It is assumed that the cloud provider provides an Infrastructure as a Service (IaaS), that delivers the storage, hardware and networking components on a virtualisation platform (e.g., KVM, Xen, etc.).

\subsection{Recommendations and conclusion}

Recommendations for a HEMP provider are given based on the vulnerabilities discovered from the threat model and the review of hardware security technologies based on TEE. Some of these recommendations reflect the challenges the security community and the hardware manufacturers are facing today, thus implementation details are still unexplored. Therefore, this chapter limits the thoroughness of recommendations to a problem description and possible approaches that need to be adapted for the HEMS. The problem description is linked to the identified requirements and threats, where the approaches are linked to the hardware technology review. The list of recommendations is as follows:

- The HEMP should support isolation of services in terms of data and resources: At the service layer envisioned for the HEMS, software frameworks for energy management already provide isolation of services (e.g., OSGi-based software frameworks). However, as services become mission critical in relation to energy management, the computational resources must also be isolated. Furthermore, as services need different privileges for accessing data, the HEMS should provide a data isolation mechanism. An application isolation mechanism based on the TyTAN security architecture [28] could comply with such demands. Furthermore, it allows the use of real-time-dependent intelligent automation 
services and allows for securely installing additional communication drivers (see R1, R2, R6, R7 R9, R10, R11).

- The HEMP could provide an inverse sandboxing mechanism, if the deployment of a service is sensitive for access or modification by highest privileged users: Acting as a platform for intelligent automation services to be deployed on, the service provider might have contractual agreement with the residential consumer about their electricity consumption. For achieving this, the service providers must ensure the integrity and confidentially of the intelligent automation services. This can be achieved, for example, by using the inverse sandboxing mechanism that the Haven [91] security architecture provides (see R5).

- The HEMP should place the device authentication process and the update process in a trusted environments and vulnerable data in a secure data storage (e.g., containing private keys) Authentication becomes a larger problem in the smart grid because of the necessity of self-organisation. A possible solution is presented in [31] which is based on TEE (see R3, R4, R7, R8).

Constructing a HEMP, which is both secure and ensures the privacy of considered data assets, is challenging. But in order to do so, an important property for enforcing security is to build it in the system and not onto the system. This chapter contributes with a threat modelling of HEMS based on the requirement and design phases of the Microsoft Security Development Lifecycle. A domain model is constructed using UML, and the requirements are elicited from use cases for HEMS under research. A DFD models an abstract HEMS platform based on a combination of the architecture of OGEMA framework and reference architecture of a mobile platform [32]. Based on a threat analysis of the DFD, an attack tree is constructed with the focus on a malicious service attacker. Given the threats, mitigation strategies are reviewed for giving recommendations for HEMP manufacturers in the future smart grid.

\section{Acknowledgements}

The research leading to these results has received funding from the EU Seventh Framework Programme (FP7/2007-2013) under grant agreement no. 317761 (SmartHG).

\section{Author details}

Søren Aagaard Mikkelsen and Rune Hylsberg Jacobsen*

*Address all correspondence to: rhj@eng.au.dk

Department of Engineering, Aarhus University, Denmark 


\section{References}

[1] T. H. Christensen, A. Ascarza, and W. Throndsen, Country-specific factors for thedevelopment of household smart grid solutions: Comparison of the electricity systems, energypolicies and smart grid RED and demonstration projects in Spain, Norway and Denmark. København: SBIforlag, 2013.

[2] P. Palensky and D. Dietrich, "Demand Side Management: Demand Response, Intelligent Energy Systems, and Smart Loads," Ind. Informatics, IEEE Trans., vol. 7, no. 3, pp. 381-388, 2011.

[3] A. Shostack, Threat modeling: Designing for security. Indianapolis, IN: Wiley, 2014.

[4] Object Management Group (OMG), “Unified Modeling Language.” [Online]. Available from: http://www.omg.org/spec/UML/ [Accessed: 30-Jan-2016].

[5] M. Zillgith, F. Institut, S. Energiesysteme, and D. Nestle, "Security Architecture of the OGEMA 2.0 Home Energy Management System System Concept and Security Requirements," Int. ETG-Kongress, vol. 9, pp. 1-6, 2013.

[6] N. Geoffray, G. Thomas, G. Muller, P. Parrend, S. Frenot, and B. Folliot, "I-JVM: A Java Virtual Machine for Component Isolation in OSGi," 2009 IEEE/IFIP International Conference on Dependable Systems \& Networks, pp. 544-553, 2009.

[7] A. Kanuparthi, R. Karri, and S. Addepalli, "Hardware and Embedded Security in the Context of Internet of Things," in Proceedings of the 2013 ACM workshop on Security, Privacy \& Dependability for Cyber Vehicles - CyCAR '13, pp. 61-64, 2013.

[8] J. Zalewski, S. Drager, W. McKeever, and A. J. Kornecki, “Threat Modeling for Security Assessment in Cyberphysical Systems," Proceedings of the Eighth Annual Cyber Security and Information Intelligence Research Workshop on - CSIIRW '13, 2013, p. 1.

[9] T. Lu, J. Lin, L. Zhao, Y. Li, and Y. Peng, "A Security Architecture in Cyber-Physical Systems: Security Theories, Analysis, Simulation and Application Fields," Int. J. Secur. Its Appl., vol. 9, no. 7, pp. 1-16, 2015.

[10] K. Beckers, S. Faßbender, and M. Heisel, A threat analysis methodology for smart home scenarios, vol. 8448, no. 256980. Cham: Springer International Publishing, 2014.

[11] M. Deng, K. Wuyts, R. Scandariato, B. Preneel, and W. Joosen, “A Privacy Threat Analysis Framework: Supporting the Elicitation and Fulfillment of Privacy Requirements," Requir. Eng., vol. 16, no. 1, pp. 3-32, 2011.

[12] F. Gangale, A. Mengolini, and I. Onyeji, "Consumer Engagement: An Insight from Smart Grid Projects in Europe," Energy Policy, vol. 60, pp. 621-628, 2013.

[13] R. H. Jacobsen and S. A. Mikkelsen, "Infrastructure for Intelligent Automation Services in the Smart Grid," Wirel. Pers. Commun., vol. 76, no. 2, pp. 125-147, 2014. 
[14] S. A. Mikkelsen and R. H. Jacobsen, "Consumer-Centric and Service-Oriented Architecture for the Envisioned Energy Internet," in 2015 Euromicro Conference on Digital System Design, 2015, pp. 301-305.

[15] Microsoft - Developer Network, "Chapter 1: Security Fundamentals for Web Services." [Online]. Available from: https://msdn.microsoft.com/en-us/library/ff648318.aspx [Accessed: 25-Jan-2016].

[16] E. S. K. Yu, “Towards Modelling and Reasoning Support for Early-Phase Requirements Engineering," in Proceedings of ISRE '97: 3rd IEEE International Symposium on Requirements Engineering, 1997, pp. 226-235.

[17] Home Gateway Initiative (HGI), “Use Cases and Architecture for a Home Energy Management Service", 2011 [Online].Available: http://tinyurl.com/hposzxd .

[18] Energy@home, “Use cases V 3.0,” 2015 [Online]. Available: http://tinyurl.com/hfctt9e

[19] U.S. National Institute of Standards and Technology, Guidelines for smart grid cybersecurity. Gaithersburg, MD, 2014.

[20] C. Greer, D. A. Wollman, D. E. Prochaska, P. A. Boynton, J. A. Mazer, C. T. Nguyen, G. J. FitzPatrick, T. L. Nelson, G. H. Koepke, A. R. Hefner Jr, V. Y. Pillitteri, T. L. Brewer, N. T. Golmie, D. H. Su, A. C. Eustis, D. G. Holmberg, and S. T. Bushby, "NIST Framework and Roadmap for Smart Grid Interoperability Standards, Release 3.0," Nist Spec. Publ., vol. 0, Gaithersburg, pp. 1-90, Oct. 2014

[21] M. Pichler, A. Veichtlbauer, and D. Engel, "Evaluation of OSGi-based architectures for customer energy management systems," 2015 IEEE Int. Conf. Ind. Technol., vol. 0, pp. 24552460, Mar. 2015.

[22] S. Checkoway and H. Shacham, "Iago attacks," in Proceedings of the eighteenth international conference on Architectural support for programming languages and operating systems - ASPLOS '13, 2013, vol. 41, no. 1, p. 253.

[23] A. Vasudevan, E. Owusu, Z. Zhou, J. Newsome, and J. M. McCune, "Trustworthy Execution on Mobile Devices: What Security Properties Can My Mobile Platform Give Me?," Lect. Notes Comput. Sci. (including Subser. Lect. Notes Artif. Intell. Lect. Notes Bioinformatics), vol. 7344 LNCS, pp. 159-178, 2012.

[24] ARM Security Technology, "Building a Secure System using TrustZone Technology." [Online]. Available from: http://infocenter.arm.com/help/topic/com.arm.doc.prd29genc-009492c/PRD29-GENC-009492C_trustzone_security_whitepaper.pdf [Accessed: 28-Jan-2016].

[25] Intel, Software guard extensions programming reference, 2013. [Online]. Available from: https://software.intel.com/sites/default/files/329298-001.pdf [Accessed: 27-Jan-2016].

[26] V. Costan and S. Devadas, Intel SGX explained, 2016. [Online]. Available from: http:// ia.cr/2016/086. 
[27] P. Koeberl, S. Schulz, A.-R. Sadeghi, and V. Varadharajan, “TrustLite: A Security Architecture for Tiny Embedded Devices," Proc. Ninth Eur. Conf. Comput. Syst. EuroSys '14, vol. 0, pp. 1-14, 2014.

[28] F. Brasser, B. El Mahjoub, A. Sadeghi, C. Wachsmann, and P. Koeberl, “TyTAN: Tiny Trust Anchor for Tiny Devices," in Proceedings of the 52nd Annual Design Automation Conference on - DAC '15, 2015, pp. 1-6.

[29] A. Baumann, M. Peinado, and G. Hunt, "Shielding Applications from an Untrusted Cloud with Haven," ACM Trans. Comput. Syst., vol. 33, no. 3, pp. 1-26, 2015.

[30] Microsoft Research, “Drawbridge." [Online]. Available from: http://research.microsoft.com/en-us/projects/drawbridge/ [Accessed: 28-Jan-2016].

[31] A. J. Paverd and A. P. Martin, "Hardware Security for Device Authentication in the Smart Grid," Lect. Notes Comput. Sci. (including Subser. Lect. Notes Artif. Intell. Lect. Notes Bioinformatics), vol. 7823 LNCS, pp. 72-84, 2013.

[32] N. Asokan, L. Davi, A. Dmitrienko, S. Heuser, K. Kostiainen, E. Reshetova, and A.-R. Sadeghi, "Mobile Platform Security," Synth. Lect. Inf. Secur. Privacy, Trust, vol. 4, no. 3, pp. 1-108, 2013. 



\section{Section 5}

Economical Optimization of Operational Cost for Micro-Grids 

Chapter 10

\title{
Energy Management and Economic Operation Optimization of Microgrid under Uncertainty
}

\author{
Shouxiang Wang, Leijiao Ge, Kai Wang and \\ Shengxia Cai
}

Additional information is available at the end of the chapter

http://dx.doi.org/10.5772/63802

\begin{abstract}
Microgrid provides an effective means to promote renewable energy utilization via deploying multiple distributed generations (DGs) with energy storage systems (ESSs), loads, control devices and protect devices, which can operate in either islanded mode or grid-connected mode. In order to coordinate the output of different DGs and realize the potential of renewable energy, energy management and economic dispatch of microgrid is needed. Both distributed energy resources (DERs) and user loads in microgrid have uncertainty characteristics; so the randomness of the wind speed and solar radiation intensity are modeled by interval mathematics and the interval output of the wind turbine and photovoltaic (PV) generation system are obtained. Then, a microgrid economic optimization model based on interval optimization method is proposed. Next, combined with the time-of-use characteristic, issue of the power exchange with the external grid has been considered. Finally, Considering the effect of ESS, this chapter discusses the impacts of uncertainty of renewable energy power and load power on optimization results, as well as the effects of the degree of load uncertainty or load fluctuation on scheduling results. The results verify the robustness and effectiveness of the proposed method in dealing with uncertainty optimization problem of microgrid.
\end{abstract}

Keywords: economic operation optimization, energy management, microgrid, uncertainty, distributed generation

\section{Introduction}

With the increasing depletion of fossil fuels, the deterioration of global environment and the dependence of human society on energy, the utilization of renewable energy sources (RESs), 
such as wind energy, solar energy, and so on, has been paid more and more attention. Distributed generation (DG) is raising high interests in distribution systems due to the deregulation and environmental concerns, the unique features such as the flexible utilization of dispersed RESs, and the flexible generating strategy. However, single DG cannot provide high-quality and sustainable power. The implementation of dispersed DGs, however, may bring additional challenges while providing various benefits [1]. To make full use of renewable energy, the concept of microgrid came into existence.

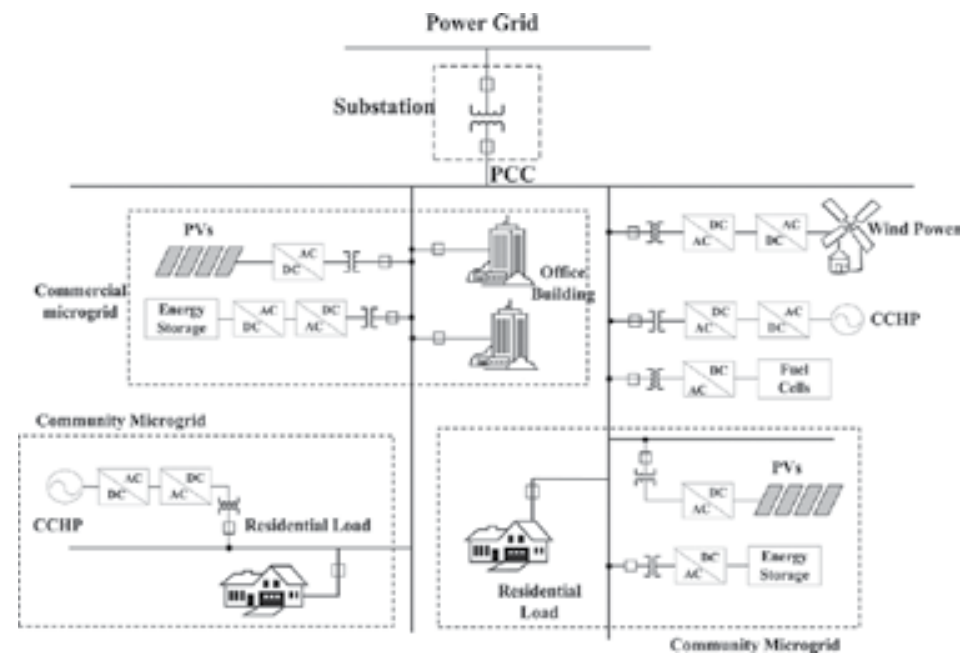

Figure 1. Typical structure of microgrid.

Microgrid is a section of distribution system that contains distributed energy resources (DERs) and can be isolated from the rest of the network when contingency occurs in upstream grid. The ability to operate in islanded mode could potentially enhance the local reliability. Furthermore, microgrid can adopt multiple DGs and the optimal deployment of different types of DGs may complement the intermittent nature of DERs so as to promote the renewable energy utilization [2]. Microgrid usually consists of DGs, ESSs, loads, control devices, and protect devices, etc. It is an autonomous, self-manage, and self-control system, which can operate in either islanded mode or grid-connected mode. In order to coordinate the output of different DGs and realize the potential of renewable energy, the economic dispatch of microgrid is needed [3]. Figure 1 depicts a typical structure of microgrid.

From Figure 1, it can be seen that DGs in microgrid mainly contain wind power generation system [4], photovoltaic (PV) system, combined cooling heating and power (CCHP) and fuel cell (FC). The loads in microgrid consist of the power load and the cold and hot load of residential users and office buildings. The energy storage devices in microgrid consist of DC and AC energy storage, and battery storage is mainly applied [5]. Meanwhile, microgrid includes a number of energy conversion devices, which are mainly AC/DC conversion devices. In general, microgrid operates in grid-connected mode, connecting with the external distri- 
bution system through a static switch as the point of common coupling (PCC). When the PCC disconnects, microgrid turns into islanded operation mode and the internal loads are totally supplied by DGs in microgird. A large microgrid may consist of several smaller ones, which can also operate independently and supply the corresponding loads, respectively. For instance, some small commercial microgrids consist of PV and AC energy storage devices are located around the modern high buildings. Moreover, many small community microgrids consist of gas turbine, and PV and DC energy storage devices are located around residential loads.

Besides the satisfying of electricity demand, microgrid can also meet the cold and hot load demand. The generation units within microgrid can be installed near users, thus the costs of transmission and distribution are reduced, and the utilization efficiency of energy is improved. DGs are easy to identify installing locations, and the installation periods are usually short. In addition, microgrid has advantages of clean energy utilization, low noise, and so on. Therefore, it is significant to do research on the economic operation of microgrid.

As energy conservation and loss reduction are realized through energy dispatch of generators in a conventional power system, the economic and efficient operation of microgrid is realized through energy management and economic operation of microgrid. Compared with a conventional power system, controlled variables in microgrid are much more abundant such as the active power of DGs, the voltage of voltage-type inverter, the current of current-type inverter, the active power of energy storage devices, the reactive power compensation of adjustable capacitors, and the proportion of thermal loads and electricity loads in CCHPs. Considering the normal operation constraints of power system, certain benefits can be realized by adjusting controlled variables such as the properly dispatch of energy and the maximum utilization of renewable energy in microgrid so as to guarantee the economic operation of microgrid. Meanwhile, when microgrid operates especially with high penetration, the energy loss of transformers and feeders in distribution system can be reduced through the effective control of microgrid output.

This chapter mainly studies the characteristics of various DGs, establishes mathematical models, analyzes different operational control strategies, and proposes an economic operation optimization method considering uncertainties of DGs in microgrid [6-7]. The DERs and user loads in microgrid both have the uncertainty characteristics, so it is worth to conduct an indepth study on how to consider the effect brought by these uncertainty factors in economic optimization of microgrid [8-9]. In order to characterize these uncertainties in microgrid, the randomness of the wind speed and solar radiation intensity are described by interval and then the output prediction interval value of the wind turbine and PVs are obtained. Combined with interval description of load uncertainty, a microgrid economic optimization model based on interval optimization method is proposed [10]. Then combined with the time-of-use characteristic, issue of the power exchange with the external grid has been considered. Finally, take the effects analysis of storage on the economic operation of the system as an example, this chapter has discussed the impacts of uncertainty of renewable energy power and load power on optimization results, as well as the effects of the degree of load uncertainty or load fluctuation on scheduling results. The results verify the robustness of the proposed method and model, and show the effectiveness in dealing with uncertainty optimization problem. 


\section{Modeling of DGs in microgrid under uncertainty}

\subsection{Uncertainty model for PV output}

PV arrays can convert solar radiation into DC power and then access into AC power via PV inverters. For a PV array, its maximum DC power output can be calculated via Eq. (1). In Eq. (1), the area of the PV array $A_{P V}$ is fixed for a specific PV power generation system. In addition, PV inverters are usually operated in the maximum power point tracking (MPPT) mode with relatively constant power conversion efficiency $\eta$. On the other hand, the operation temperature of solar panels $T_{c}$ and the solar radiation on panels $G_{T}$ are varied. Several factors, such as the ambient air temperature, the atmospheric pressure, and the wind speed, may impact the operation temperature of solar panels. The operation temperature of solar panels can be calculated via the ambient temperature in Eq. (2). Substituting Eq. (2) into Eq. (1), the output power of a PV array can be calculated in Eq. (3). Eq. (3) shows that the power output of a PV cell is mainly determined by the solar radiation and the ambient temperature. The ambient temperatures may not change dramatically in a very short time period. Thus, the cloud cover is considered as the dominant uncertainty factor that affects the PV output;

$$
\begin{gathered}
P=\eta A_{P V} G_{T}\left[1-0.005\left(T_{C}-25\right)\right] \\
T_{c}=T_{a}+C G_{T} \\
P=\eta A_{P V} G_{T}\left[1-0.005\left(T_{a}+C G_{T}-25\right)\right]
\end{gathered}
$$

An interval cloud model is introduced to describe uncertainties of the cloud cover. The solar radiation effect on the panels can be calculated by the solar radiation outside the atmosphere and the corresponding cloud cover index as shown in Eq. (4) and Table 1:

$$
\left[G_{T}\right]=[I] \cdot G_{a}
$$

\begin{tabular}{ll}
\hline Weather & Cloud cover index \\
\hline Rainy, snowy & {$[0.1,0.2]$} \\
Overcast & {$[0.2,0.3]$} \\
Overcast to cloudy & {$[0.3,0.5]$} \\
Cloudy & {$[0.5,0.7]$} \\
Cloudy to clear & {$[0.7,0.9]$} \\
Clear & {$[0.9,1.0]$} \\
\hline
\end{tabular}

Table 1. Cloud cover index. 
Given the interval cloud cover model in Eq. (4), the interval PV output model can be formulated via the following two steps:

a. Predict the next-day solar radiation outside the atmosphere at the PV location. Based on the weather forecast and the solar radiation forecast, the solar radiation interval is calculated as $\left[\underline{G}_{T}, \bar{G}_{T}\right]$ via Eq. (4).

b. Obtain the interval of the PV output $\left[P\left(\underline{G}_{T}\right), P\left(\bar{G}_{T}\right)\right]$ using the PV power output function via Eq. (3).

\subsection{Uncertainty model for WTG output}

Wind turbine generators (WTGs) can convert kinetic energy from wind into electricity. The mechanical power generated by the wind turbine $P_{m}$ can be calculated via Eq. (5);

$$
P_{m}=0.5 \rho A V^{3} C_{P}
$$

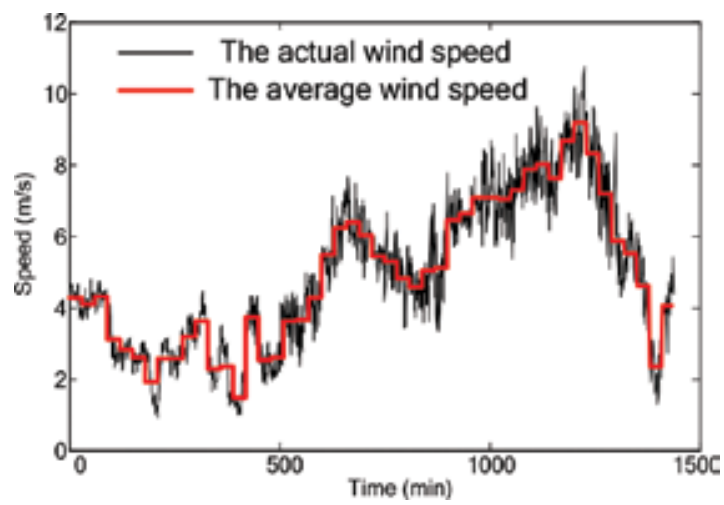

Figure 2. Wind speed variety during one day.

where $\rho$ is the air density, $A$ is the air cross-section, $V$ is the wind speed, and $C_{P}$ is a function of the speed ratio and the blade pitch angle.

The real power injected into electric power systems by a WTG is mostly affected by wind speed. Figure 2 shows a one-day wind speed profile of a wind farm located in Qindao, China. It illustrates that wind speed varies drastically in a single day as shown in the black line. Because accurate forecast on instantaneous wind speed is difficult, average wind speeds for each 30 minutes are usually used to approximate wind power outputs as shown in the red curve.

As the wind power is proportional to the cubic of wind speed, the wind speed forecast error would lead to considerable errors. To accurately quantify uncertainties of wind speed, an interval WTG output model is formulated as follows:

a. Predict the wind speed interval $\left[V_{T}\right]=\left[\underline{V}_{T}, \bar{V}_{T}\right]$ for the next day. 
b. The wind power output interval is calculated as $\left[P\left(\underline{V}_{T}\right), P\left(\bar{V}_{T}\right)\right]$ via Eq. (5).

\section{Energy management and economic operation strategy of microgrid}

Microgrid has the merits of environmental friendliness and economical efficiency. Its environmental friendliness is guaranteed by the utilization of DERs. Its economical efficiency will be guaranteed by energy management and economic operation of microgrid. Economic operation optimization of microgrid is a nonlinear combination optimization problem with multiple variables and multiple constraints, which determines the optimal dispatch scheme of microgrid to achieve the best economic benefit according to the operating costs, parameters, and types of DGs and other components under the precondition of satisfying the load demand of users and operation constraints.

The operational economy of the microgrid is directly related to the interests of the users and the main power grid, and the proper economic operation control strategy is particularly important. The operation control strategy of microgrid has its own features compared with conventional power grid. In a conventional grid, the key to economic operation control is the optimal dispatching of combined fire and hydraulic power plants. And environmental factors are seldom considered. However, a microgrid may comprise many types of DGs, such as the wind turbine and PV, the output power of which varies with the changing of environment. Hence, economic operation of microgrid must consider environmental impact.

The operation mode of microgrid is divided into grid-connected mode and isolated island mode, and the operational control strategies faced by different operation modes should also be different. In grid-connected operation, there is a power exchange between microgrid and distributed power network. External power grid provides a backup to supplement the shortage of electricity or absorb the excess electricity generated by microgrid. Not all loads in microgrid will be supplied power by internal DGs, but only critical loads will be served by microgrid during isolated island operation mode. Faced with the shortage of supply or excess supply, there are significant differences in control strategies between the two operation modes of microgrid.

The control strategy of economic operation of microgrid is to ensure the safe and stable operation of the system under either operation mode of microgrid. When disconnected from the external power grid, the microgrid is capable of local voltage and frequency control and generates or consumes the temporary power to balance the generating power and the load power.

\subsection{Grid-connected operation characteristics of microgrid}

Microgrid exchanges the power with the external power grid through a static switch as PCC in grid-connected operation mode under normal circumstances. External power grid provides electrical auxiliary support when generating capacity of internal generating unit of microgrid cannot meet the demand of internal loads. On the contrary, external power grid need to absorb 
some of the excess power when generating capacity of generating unit is much more than load demand. Under grid-connected operation mode, microgrid system can fully utilize the law of electric power market to control the operation of DGs by the power exchange with the external power grid, which can achieve the best economic performance.

In the grid-connected operation mode, the frequency adjustment of microgrid is done by the interaction with external power grid. High penetration of DGs may cause voltage and reactive power offset or shock. Therefore, effective local voltage control is needed no matter in gridconnected mode or isolated island mode. The regulation of voltage level of microgrid is required to ensure the reliability and stability of local power supply. The voltage-reactive droop controller is an effective method to control the local voltage in microgrid.

\subsection{Isolated island operation characteristics of microgrid}

The isolated island operation mode of microgrid can be divided into intentional and unintentional islanding mode according to planning in advance or not. The so-called intentional islanding scheme will ensure the stable operation after the formation of an island before microgrid is disconnected with external power grid by proper island division according to distributed power supply capacity, load demand, and system operational state. While unintentional island refers to the case that when a serious fault occurs in the external power grid, the power quality will no longer meet the criterion and the protection device will act, which resulting in the isolation with external power grid unintentionally, the microgrid operates unstably with loads.

The transition from grid-connected operation to isolated island operation state is the primary consideration in the operation of microgrid in the island mode. In this process, the energy conversion and control system of microgrid adopts a more flexible method for frequency regulation because microgrid basically has few rotating generating units and with smaller inertia than conventional power system. Therefore once disconnected with an external power connection, microgrid should consider load shedding according to the priority of loads and to ensure the stable operation of the system when the load demand excesses a distributed power supply capacity.

\subsection{Economic operation strategy of microgrid}

At present, there are two basic control strategies in microgrid: Master-Slave operation and peer-to-peer control. Master-slave operation is mainly used in microgrid islanded operation mode, it defines a reference DG to unify the coordinated control of other DGs and maintain the balance of power within the system. Peer-to-peer control adopts the same control scheme for all the DGs and with the aid of the curves of $\mathrm{P} / \mathrm{f}$ or $\mathrm{Q} / \mathrm{U}$ and realize automatic adjustment of voltage and frequency based on control strategy of dropping of external characteristic. Various other control strategies can be considered as the improvement and integration of the two control strategies. 


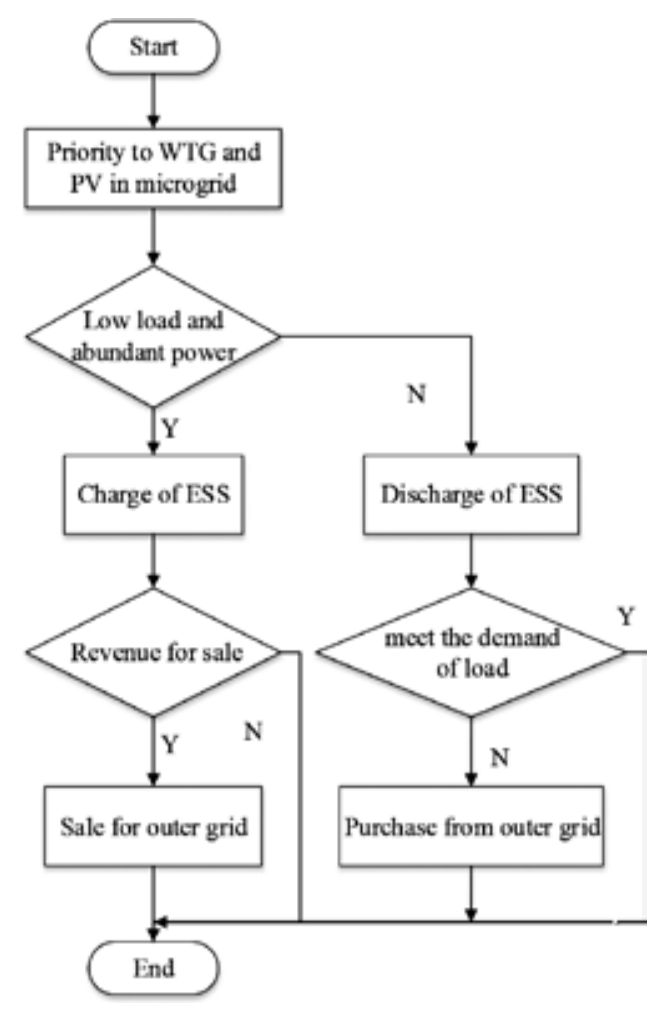

Figure 3. Flowchart of control strategy in grid-connected mode.

In terms of different operation mode, the operation control strategy is also different. In gridconnected mode, the economic operation of microgrid mainly concerns with the benefit of the power exchange with the external power grid. The scale of power exchange mainly follows the following principles: (1) given priority to DG power generations when generation costs of DGs of microgrid are generally lower than the external electricity price; (2) given priority to external power purchase when generation costs of DGs of microgrid are higher than the external electricity price; and (3) if DG power is not enough to take the whole load of microgrid, the energy storage device can be used for power supply first, the shortage will be supplemented from the external power purchase electricity. Taking into account different periods with different electricity price, the energy storage device in microgrid should give priority to charging, and then compare the cost of DG power generation and the revenue from electricity sales to determine whether sell electricity to grid.

The control strategy for each DG in microgrid in grid-connected mode is as follows, which is shown in Figure 3.

- Give priority to wind turbine and PV power generation as they are renewable and clean energy in microgrid. 
- Considering the effect of real-time electricity price, when the load is low and the power of the wind turbine and PV cells is abundant, the energy storage system (ESSs) will be charged. Only when the electricity price of power grid is higher than the price of the energy storage electricity price, the sale of electricity is considered.

- In the peak period of load, if the wind turbine, PV cells, fuel cell output is insufficient, give priority to the battery discharge, the battery does not meet the discharge limit to meet the load demand, consider the sale of electricity.

- When the battery discharge cannot meet the demand of the peak load, electricity should be purchased from the external power grid.

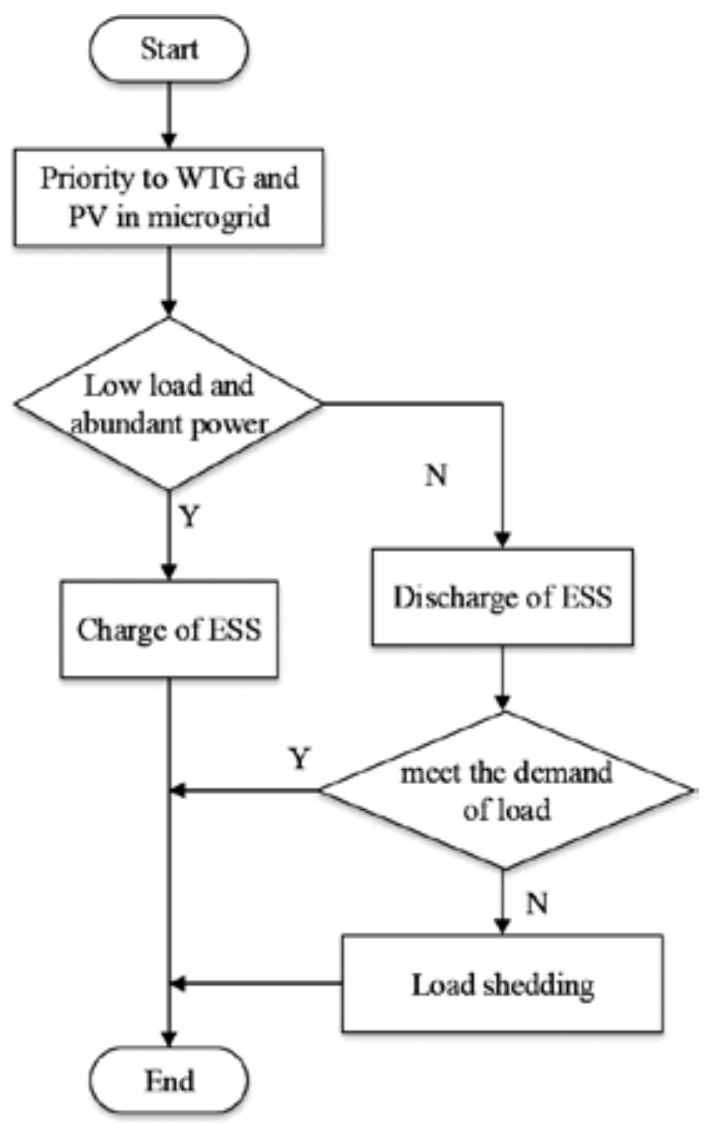

Figure 4. Flowchart of control strategy in island operation mode.

Microgrid in the island operation mode, there is no problem of power exchanging because there is no contact with the external power grid. The control strategy of DGs in microgrid in isolated island mode is as follows, which is shown is Figure 4.

- Give priority to wind turbine and PV power generation. 
- In the period of valley load, wind turbine and PV output power are abundant, the priority of energy storage system is to charge, while in the peak load period, if lack of wind turbine, PV cells, fuel cell output, priority is given to the discharge of the energy storage system.

- When the discharge of energy storage system cannot meet the demand of peak load, the load shedding should be taken into consideration.

\section{Energy management and economic operation optimization of grid- connected microgrid with DGs considering uncertainty}

The uncertainty issue of the wind turbine, PV can be modeled by the interval mathematical theory. At the same time, the interval mathematical theory can also satisfy the equation constrains for the forecast value of load and renewable energy. Thus, the interval mathematical theory can achieve better optimization operation of microgrid. This section introduces the proposed interval linear programming model for optimal operation of microgrid considering time of use electricity price. The proposed interval linear programming model can also consider the influence of weather uncertainty on the output of wind turbine and PV system.

Interval linear programming (ILP) is an effective tool for solving the uncertainty problem. ILP is a linear programming method that combines the interval mathematical method and linear programming, and can also solve these problems which object function and constraints have interval number.

The common type of interval linear programming is as follows:

$$
\begin{array}{cc}
\text { Min } & f^{ \pm}=C^{ \pm} X^{ \pm} \\
\text {S.t } \quad & A^{ \pm} X^{ \pm} \leq B^{ \pm} \\
& X^{ \pm} \geq 0
\end{array}
$$

where $\left\{\begin{array}{l}A^{ \pm}=\left\{a_{i j}{ }^{ \pm}=\left[a_{i j}, a_{i j}{ }^{+}\right] \mid \forall i, j\right\}, A^{ \pm} \in\left\{R^{ \pm}\right\}^{m \times n} \\ B^{ \pm}=\left\{b_{i}^{ \pm}=\left[b_{i}^{-}, b_{i}^{+}\right] \mid \forall i\right\}, B^{ \pm} \in\left\{R^{ \pm}\right\}^{m \times 1} \\ C^{ \pm}=\left\{c_{i}^{ \pm}=\left[c_{i}^{-}, c_{i}^{+}\right] \mid \forall i,\right\}, C^{ \pm} \in\left\{R^{ \pm}\right\}^{1 \times n}\end{array} \quad, X^{ \pm} \in\left\{R^{ \pm n \times 1}, R^{ \pm}\right.\right.$represent a series of interval numbers.

Property I: the function $f^{ \pm}=C^{ \pm} X^{ \pm}$has best object $f^{ \pm}=C^{+} X^{ \pm}$and worst object $f^{ \pm}=C^{-} X^{ \pm}$, and there is always $C^{-} X^{ \pm} \leq f^{ \pm} \leq C^{+} X^{ \pm} \forall f^{ \pm}=C^{ \pm} X \pm\left(C \in\left[C^{-}, C^{+}\right]\right)$.

Property II: for the following interval in-equation: $\sum_{j=1}^{n} a_{j} \pm x_{j} \leq b^{ \pm}$, assume $P_{t}=P\left(\sum_{j=1}^{n} a_{j}^{ \pm} x_{j} \leq b^{ \pm}\right)$ 


$$
\begin{aligned}
& \Omega_{L}=\left\{\left(x_{1}, x_{2}, \mathrm{~L}, x_{n}\right) \mid \sum_{j=1}^{n} a_{j}^{+} x_{j} \leq b^{-}, \Omega_{L}{ }^{\prime}=\left\{\left(x_{1}, x_{2}, \mathrm{~L}, x_{n}\right) \mid \sum_{j=1}^{n} a_{j}{ }^{+} x_{j} \leq b^{-}\right\}\right\} \\
& \Omega_{U}=\left\{\left(x_{1}, x_{2}, \mathrm{~L}, x_{n}\right) \mid \sum_{j=1}^{n} a_{j}^{-} x_{j} \leq b^{+}, \Omega_{U}{ }^{\prime}=\left\{\left(x_{1}, x_{2}, \mathrm{~L}, x_{n}\right) \mid \sum_{j=1}^{n} a_{j}{ }^{-} x_{j} \leq b^{+}\right\}\right\} \\
& L_{t}=\operatorname{len}\left(\sum_{j=1}^{n} a_{j}^{ \pm} x_{j}\right)+\operatorname{len}\left(b^{ \pm}\right)-\max \left(0, \sum_{j=1}^{n} a_{j}^{+} x_{j}-b^{-}\right)
\end{aligned}
$$

The following result can be obtained:

1. if $P_{t}=1$, then $\left(x_{1}, x_{2}, \ldots, x_{n}\right)$ is a solution of the interval in-equation. $\left(x_{1}, x_{2}, \ldots, x_{n}\right)$ satisfies $\left(x_{1}, x_{2}, \cdots, x_{n}\right) \in \Omega_{L}$, in other format $a_{j} \in\left[a_{j}^{-}, a_{j}{ }^{+}\right], b_{j} \in\left[b_{j}{ }^{-}, b_{j}{ }^{+}\right],\left(x_{1}, x_{2}, \cdots, x_{n}\right)$ always be a solution of the interval in-equation.

2. If $0<P_{t}<1$ or $L_{t}=0,\left(x_{1}, x_{2}, \cdots, x_{n}\right)$ is a weak solution of the interval in-equation. At this time $\left(x_{1}, x_{2}, \cdots, x_{n}\right) \in \Omega_{S}, \Omega_{S}=\left(\Omega_{U} \cap \Omega_{L}{ }^{\prime}\right)$, there has $a_{j}^{*} \in\left[a_{j}^{-}, a_{j}^{+}\right], b_{j}^{*} \in\left[b_{j}^{-}, b_{j}{ }^{+}\right],\left(x_{1}, x_{2}, \cdots, x_{n}\right)$ is a solution of the interval in-equation. However, for the following condition

$$
a_{j}>a_{j}^{*} \text { or } b<b^{*}\left(a_{j} \in\left[a_{j}^{-}, a_{j}^{+}\right], b_{j} \in\left[b_{j}^{-}, b_{j}^{+}\right]\right)
$$

The interval in-equation has no result.

3. If $L_{t}<0$, then $\left(x_{1}, x_{2}, \cdots, x_{n}\right)$ is not a solution of the interval in-equation, at this time

$$
\left(x_{1}, x_{2}, \mathrm{~L}, x_{n}\right) \in \Omega_{U}{ }^{\prime}
$$

By analyzing the close relationship of the above parameters, control variable, object function and constrains, the proposed problem can be solved by two steps:

1. Obtain the result of the model $f^{-}$

$$
\begin{aligned}
& \text { Min } f^{-}=\sum_{j=1}^{k_{1}} c_{j}^{-} x_{j}^{-}+\sum_{j=k_{1}+1}^{n} c_{j}^{-} x_{j}^{+} \\
& \text {S.t: } \\
& \sum_{j=1}^{k_{1}}\left|a_{i j}\right|^{+} \operatorname{Sign}\left(a_{i j}^{+}\right) x_{j}^{-}+\sum_{j=k_{1}+1}^{n}\left|a_{i j}\right|^{-} \operatorname{Sign}\left(a_{i j}^{-}\right) x_{j}^{+} \leq b_{i j}^{ \pm} \\
& x_{j}^{ \pm} \geq 0 \quad \forall i j
\end{aligned}
$$


where $x_{j}^{ \pm}, j=1,2, \ldots, k_{1}$ are the positive interval variables of the object function. $x_{j}^{ \pm}, j=k_{1}+1, k_{1}+2, \ldots, n$ are the negative interval variables of the object function. Solving the above Eq. (8), the results can be obtained:

$$
x_{j \text { opt }}^{-}\left(j=1,2, \ldots, k_{1}\right), x_{j \text { opt }}^{+}\left(j=k_{1}+1, k_{1}+2, \ldots, n\right) .
$$

2. Obtain the result of the model $f^{+}$

$$
\begin{aligned}
& \text { Min } f^{+}=\sum_{j=1}^{k_{1}} c_{j}^{+} x_{j}^{+}+\sum_{j=k_{1}+1}^{n} c_{j}^{+} x_{j}^{-} \\
& \text {S.t : } \\
& \sum_{j=1}^{k_{1}}\left|a_{i j}\right|^{-} \operatorname{Sign}\left(a_{i j}^{-}\right) x_{j}^{+}+\sum_{j=k_{1}+1}^{n}\left|a_{i j}\right|^{+} \operatorname{Sign}\left(a_{i j}^{+}\right) x_{j}^{-} \leq b_{i j}^{ \pm} \\
& x_{j}^{ \pm} \geq 0 \quad \forall i j \\
& x_{j}^{+} \geq x_{j \text { opt }}^{-}, \quad j=1,2, \ldots, k_{1} \\
& x_{j}^{-} \leq x_{j}^{+} \text {opt }, \quad j=k_{1}+1, k_{1}+2, \ldots, n
\end{aligned}
$$

In a similar way, by solving Eq. (9) we can obtain the following result: $x_{j}{ }^{+}$opt $(j=1,2, \ldots, k), x_{j}^{-}$opt $\left(j=k_{1}+1, k_{1}+2, \ldots, n\right)$. Then, the final object value can be obtained:

$$
f^{ \pm}{ }_{o p t}=\left[f_{o p t}^{-}, f_{o p t}^{+}\right]
$$

and

$$
x_{j \text { opt }}^{ \pm}=\left[x_{j \text { opt }}^{-}, x_{j \text { opt }}^{+}\right] \text {. }
$$

Considering the variety of the uncertainty problem, using interval linear programming algorithm can build a model which includes battery, wind turbine, PV, and microgrids and build the optimal operation model of the microgrid. This model uses the operation cost as the object function, and can optimize the operation of a microgrid in a day.

\subsection{Object function}

If the object is to minimize the operation cost of a day, then it can be represented by the following equations:

$$
\sum_{t=1}^{T} C_{t}=\sum_{t=1}^{T}\left(C_{w d . t}+C_{p v . t}+C_{f l . t}+C_{e x . t}+C_{b t . t}\right)
$$




$$
\begin{gathered}
C_{w d . t}=c_{w d . t} \cdot P_{w d . t} \\
C_{p v . t}=c_{p v . t} \cdot P_{p v . t} \\
C_{f l . t}=c_{f l . t} \cdot P_{f l . t} \\
C_{s b . t}=c_{b t d . t} \cdot P_{b t d . t}-c_{b t c . t} \cdot P_{b t c . t} \\
C_{e x . t}=c_{b u y . t} \cdot P_{b u y . t}-c_{s e l l . t} \cdot P_{s e l l . t}
\end{gathered}
$$

where $T$ is the operation time, $C_{t}$ is the total operation cost of the system at time $t$. $C_{t}$ includes the operation cost of fuel cell, wind turbine, battery, and energy exchange cost of power system. The above items are represented by the following symbols, respectively: $C_{f l . t}, C_{w d . t}, C_{p v . t}, C_{s b . t}$ and $C_{\text {ex.t. }} . P_{\text {buy.t }}$ and $c_{\text {buy.t. }}$ represent the purchase power and energy purchase cost. $P_{\text {sell.t }}$ and $c_{\text {sell.t }}$ represent the sell power and energy sell income. $P_{w d . t} P_{p v . t}, P_{f . t}$ represent the output power of wind turbine, PV, and fuel cell at time $t . c_{w d . t}, c_{p v . t}, c_{f l . t}$ represent the operation maintenance cost of wind turbine, PV, and fuel sell. $c_{b t d . t}, c_{b t c . t}$ represent the charging and discharging cost of battery. $P_{b t d . t} P_{b t c . t}$ represent the charging and discharging power of battery at time $t$.

\subsection{Constraints}

1. The constraint of microgrid power equation:

$$
\sum_{t=1}^{m}\left(P_{w d . t}+P_{p v . t}+P_{f l . t}+P_{b t d . t}+P_{b u y . t}=P_{L . t}+P_{b t c . t}+P_{s e l l . t}\right)
$$

where $P_{L . t}$ represents the forecast power load of microgrid at time $t$ and the network loss is neglected.

2. The constraint of controllable micropower supply system:

$$
P_{i . \min } \leq P_{i} \leq P_{i, \max }
$$

where $P_{i \text { min }}, P_{i . m a x}$ represent the minimum and maximum power output of micropower supply system $i$, respectively.

3. The constraint of maximum exchange energy between microgrid and the connected distribution system: 


$$
P_{e . \min } \leq P_{e x . t} \leq P_{e . \max }
$$

where $P_{\text {ex.t }}$ is the difference between purchase electrical energy and sold electrical energy; $P_{\text {e.min }} P_{\text {e.max }}$ are the nether and upper value of exchange power between power system and microgrid.

4. The constraints of fuel cell ramp rate:

$$
\Delta t \cdot \Delta \underline{P}_{f} \leq P_{f . t+1}-P_{f . t} \leq \Delta t \cdot \Delta \bar{P}_{f}
$$

where $\Delta \overline{P_{f}}, \Delta P_{f}$ are the upper and lower ramp rates of fuel cell. $\Delta t$ is the time interval of unit time.

5. The operation constraint of battery:

The capacity of battery should satisfy the following constraints:

$$
S_{\min } \leq S O C_{t} \leq S_{\max }
$$

When SOC reaches the maximum value $\left(S_{\max }=100 \%\right)$, the controller of the battery control the battery stop charging, when $S O C$ reaches the minimum value, the controller control the battery stop discharging. And, the $S_{\min }$ is set to be $30 \%$ usually.

When consider the operation characteristic of battery, the energy capacity state should be same at the beginning and end of the dispatch process:

$$
\begin{gathered}
E_{b a t}(0)=E_{b a t}(T) \\
\left\{\begin{array}{l}
0 \leq P_{b . c h . t} \leq P_{b . c h . m a x} X_{t} \\
0 \leq P_{b . d i s c h . t} \leq P_{b . d i s c h . m a x} Y_{t} \\
X_{t} \in\{0,1\}, Y_{t} \in\{0,1\}
\end{array}\right.
\end{gathered}
$$

where $P_{b . c h . m a x} P_{b . d i s c h \text { max }}$ are the maximum charging and discharging power of battery, respectively.

The battery can only work at charging state or discharging state, therefore, the battery should meet the following constraint:

$$
\left\{\begin{array}{l}
X_{t}+Y_{t} \leq 1 \\
X_{t} \in\{0,1\}, Y_{t} \in\{0,1\}
\end{array}\right.
$$




\subsection{Numerical analysis}

The microgrid test system used is shown in Figure 5. The microgrid is connected to external distribution grid through a static switch at the point of common coupling (PCC). Wind turbine (WT), PV (PV), battery (BT), and fuel cell (FC) are included in the system. MV, LV means medium voltage, low voltage distribution grid and LD means routine load. The rated capacities of BT and FC are 300 and $100 \mathrm{kWh}$, respectively. The initial energy (the capacity at the time $t$ $=0$ ) in BTs is set $100 \mathrm{kWh}$. The maximum power rise rate and the maximum power drop rate of FC are 15 and $10 \mathrm{~kW} / \mathrm{h}$.

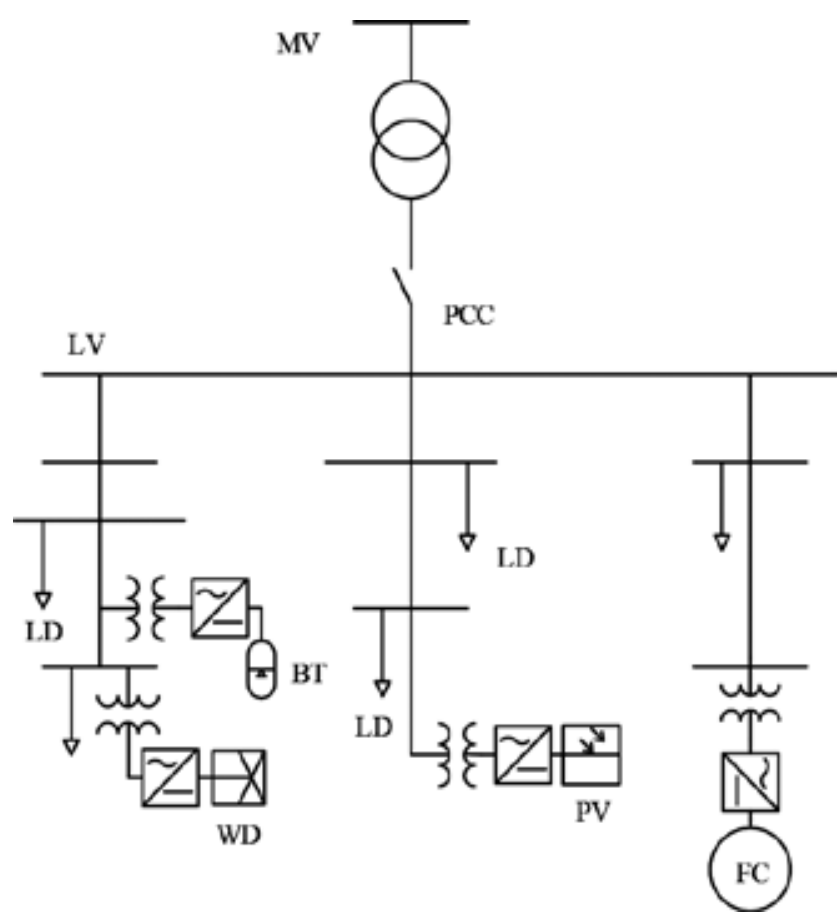

Figure 5. Architecture of a microgrid test system.

The price parameters of WT, PV, BT, and FC are shown in Table 2.

\begin{tabular}{llllll}
\hline DG & WT & PV & FC & BT & \\
\cline { 5 - 6 } & & & & Charging & Discharging \\
\hline Price $(y u a n / k W h)$ & 0.52 & 0.75 & 0.6 & 0.4 & 0.6 \\
\hline
\end{tabular}

Table 2. Price parameters.

The objective of the model is to optimize the economic cost of one-day operation of the system. Using the interval models of DG output under uncertainty and the prediction intervals of wind 
speed and illumination intensity, the prediction intervals of WT and PV power is obtained through Eqs. (1)-(4). The prediction curves are shown in Figures 6 and 7. The cloud cover index $[I]$ follows the following principle: $[I]=[0.8,1.0]$ for cloudless days, $[I]=[0.5,0.8]$ for cloudy days, $[I]=[0.1,0.3]$ for rainy and snowy days. The load prediction curve of the system is shown in Figure 8. The load curve has peak, valley, and flat at different time periods. The power electricity is bought and sold using time-of-use prices. The power price curve is shown in Figure 9.

1. The impact of BT on the system

In this case, the DG power outputs are intervals predicted as before and the load fluctuates in interval [95\%, 105\%]. The system operation costs without and with BT are compared below.

The power output intervals of FC without and with BT are shown in Figures $\mathbf{1 0}$ and 11. The bought and sold power intervals of FC without and with BT are shown in Figures 12 and 13. The one-day system operation costs are: [2431.51, 2917.86] yuan without BT, and [2357.24, 2855.32] yuan with BT. It can be seen that there exists overlap between the two cost intervals, which reflects the impact of DG uncertainty on the economic system operation. Due to the uncertainty of DG and load prediction, the comparison of system operation costs without and with BT is uncertain. However, for this system the system operation cost without BT is larger than that with BT from the aspect of interval number comparison. So it is concluded that based on the BT parameters used in this system, the participation of BT in the microgrid can decrease the system operation cost and partly improve the system economy. With different BT parameters, the conclusion may be different and even opposite, but the uncertainty analysis method still holds.

2. The impact on system operation caused by load fluctuation

Taking into consideration work and rest regime of various industries, people's life law, and weather change, load varies by $30 \%$ of the peak load between one day and night. Therefore, based on forecasting load, different levels of load fluctuation (namely, 10, 20, and $30 \%$ of load peak) are used to analyze the impact on system operation.

The impact on economic operation of microgrid caused by the uncertainty of load prediction is shown in Table 3. It can be seen from Table 3 that system operation cost is relatively stable when load fluctuation is small. With the strengthening of load fluctuation, system operation cost interval widths are also on the increase. So, the size of load fluctuation has a direct impact on the uncertainty of the system operation cost interval. 


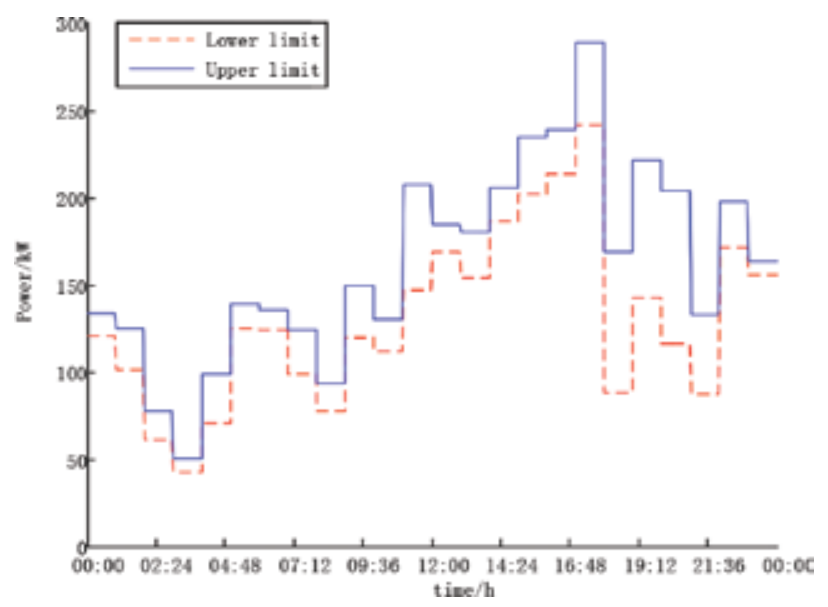

Figure 6. Prediction intervals of wind power.

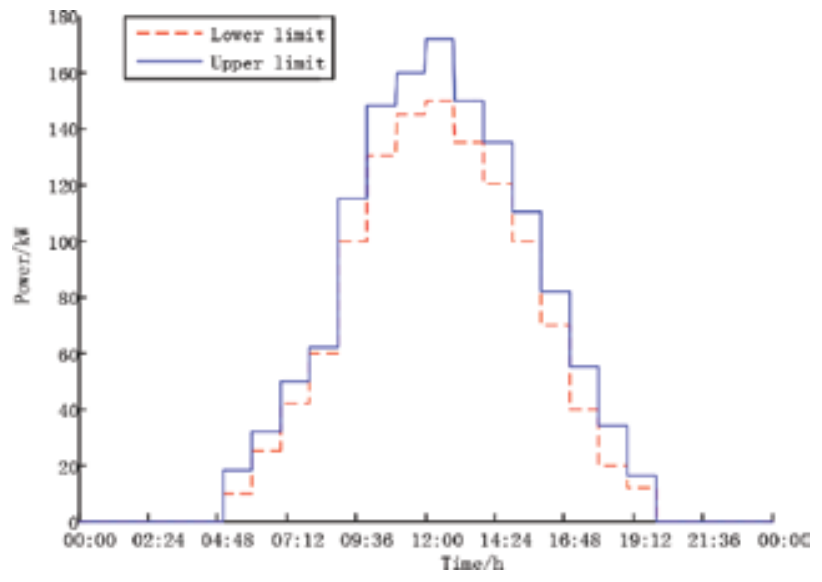

Figure 7. Prediction intervals of PV battery power.

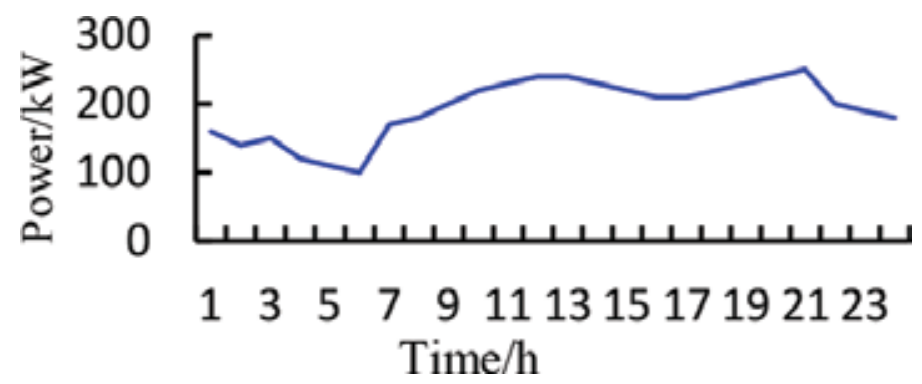

Figure 8. The load prediction curve of the system. 


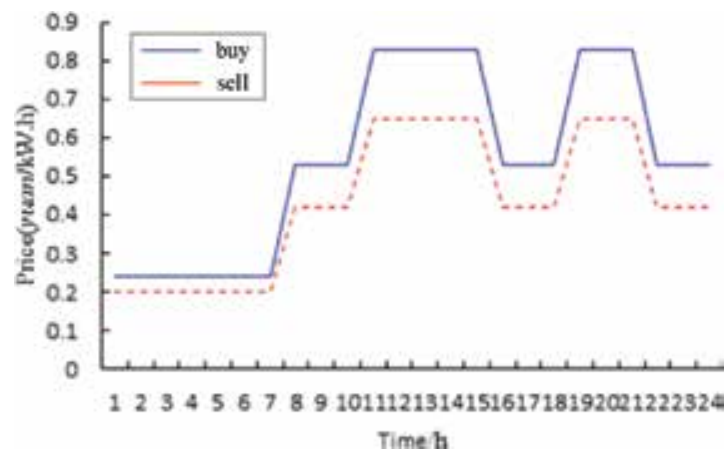

Figure 9. Power price curve.

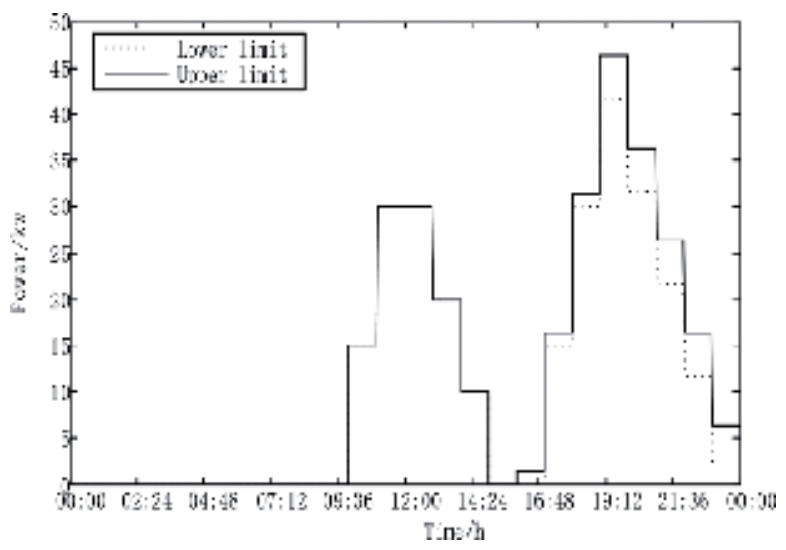

Figure 10. Power output intervals of FC without BT.

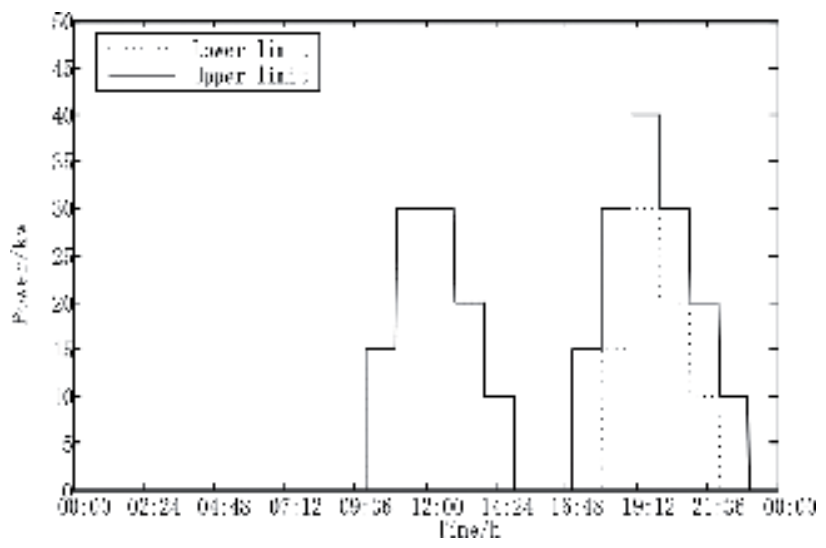

Figure 11. Power output intervals of FC with BT. 


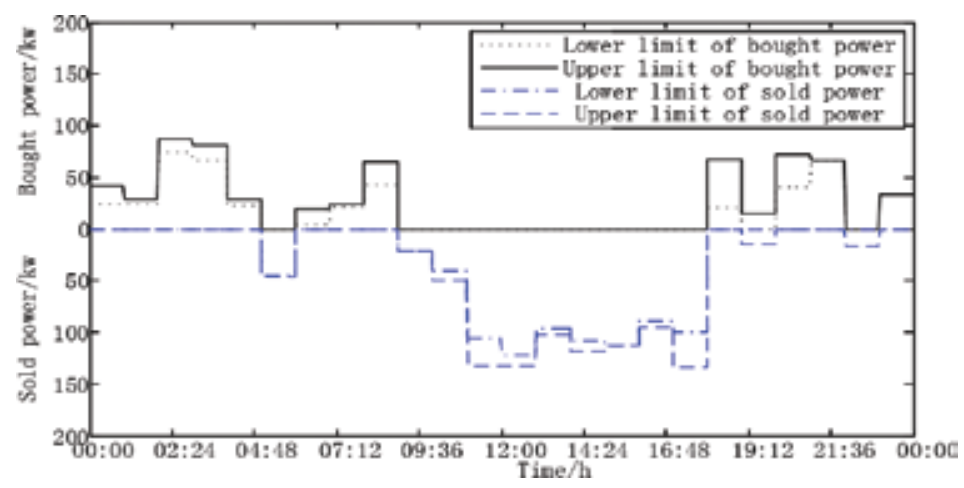

Figure 12. Bought and sold power intervals of FC without BT.

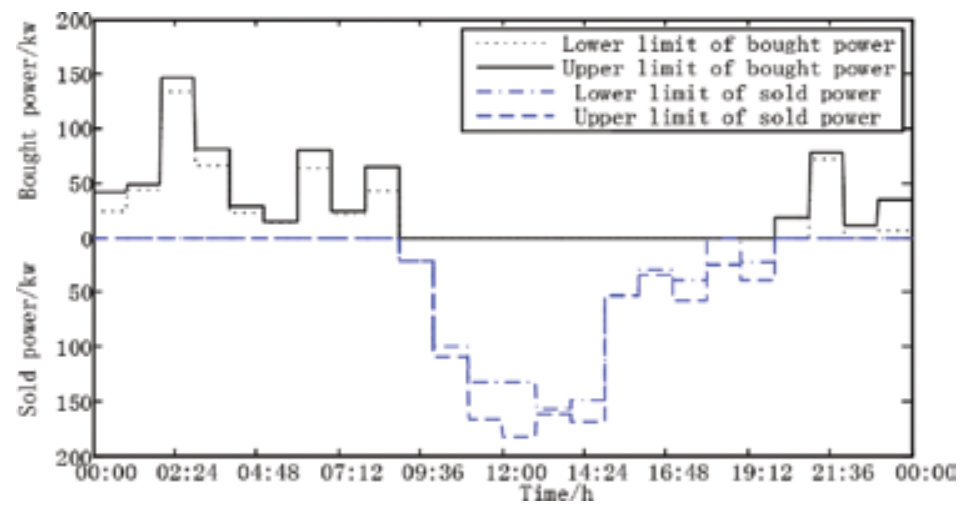

Figure 13. Bought and sold power intervals of FC with BT.

\begin{tabular}{ll}
\hline Load fluctuation $(\%)$ & System operation cost of a day (yuan) \\
\hline 0 & {$[2596.08,2624.43]$} \\
10 & {$[2357.24,2855.32]$} \\
20 & {$[2120.79,3088.71]$} \\
30 & {$[1894.29,3328.94]$} \\
\hline
\end{tabular}

Table 3. System operation cost under different load prediction fluctuation.

Figures 14-19 show battery charge and discharge power, fuel cell power output, power purchase interval, and power sale interval corresponding to $0 \%$ and $20 \%$ of load fluctuation. By comparing two figures, it can be seen load is supplied by upper grid and wind turbine and fuel cells do not work during load valley periods (00:00-6:00) because of time-of-use price. If the SOC of storage battery is less than $100 \%$, it can absorb extra power to adjust active power 
during other periods. It should be noted that power purchase interval and storage battery charging interval corresponding to $20 \%$ of load fluctuation are larger than $0 \%$. In conclusion, load fluctuation levels can have impact on power purchase interval width and storage battery charging interval width during load valley periods.

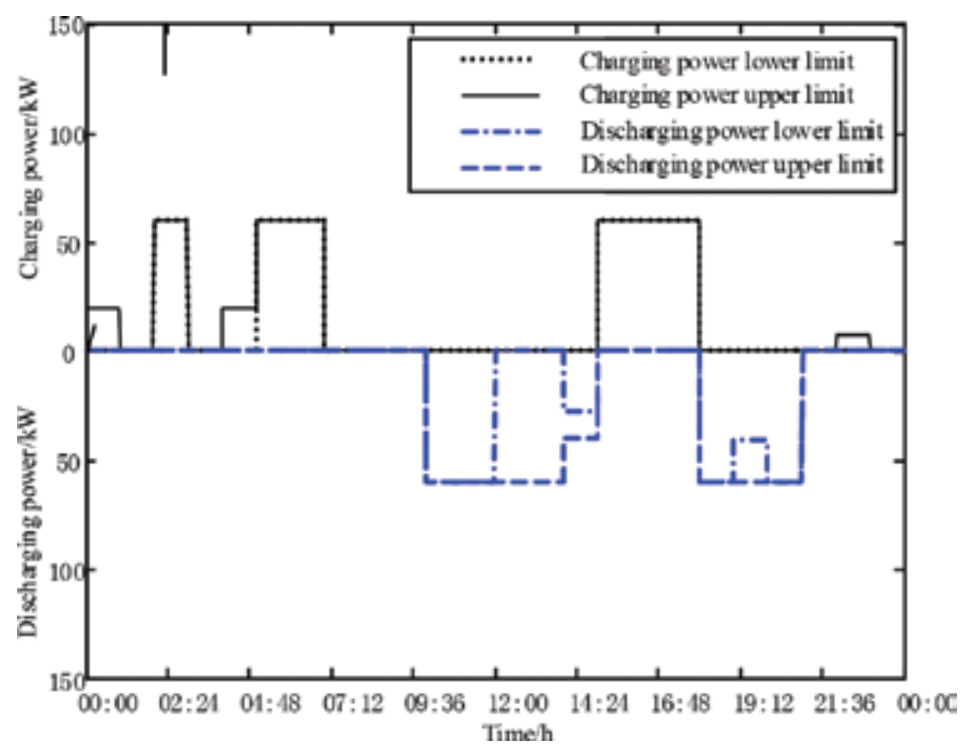

Figure 14. Storage battery charging intervals corresponding to $0 \%$ of load fluctuation.

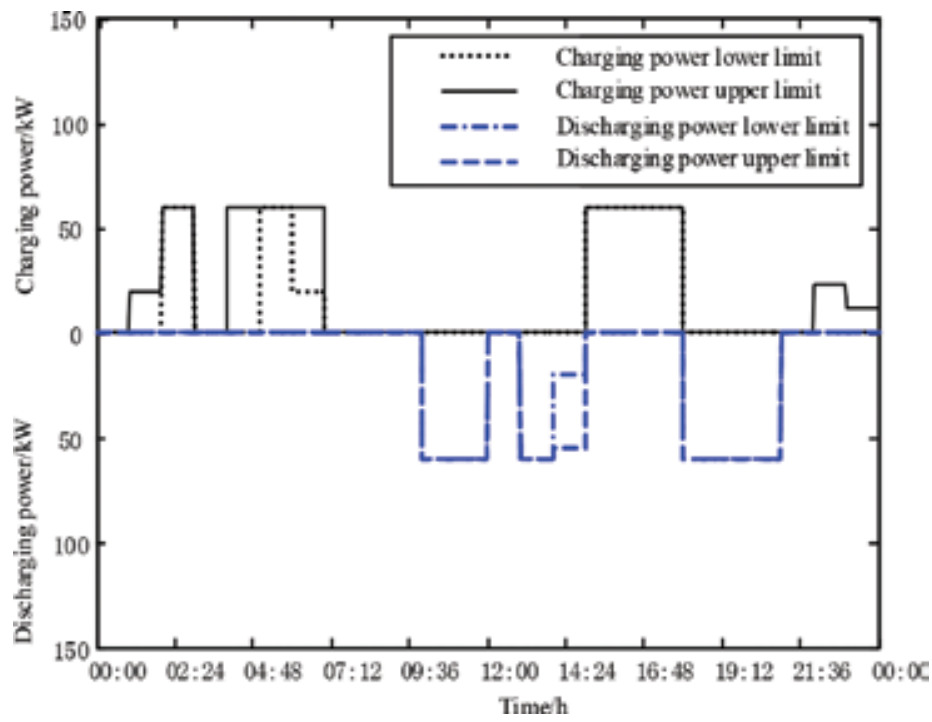

Figure 15. Storage battery charging intervals corresponding to $20 \%$ of load fluctuation. 


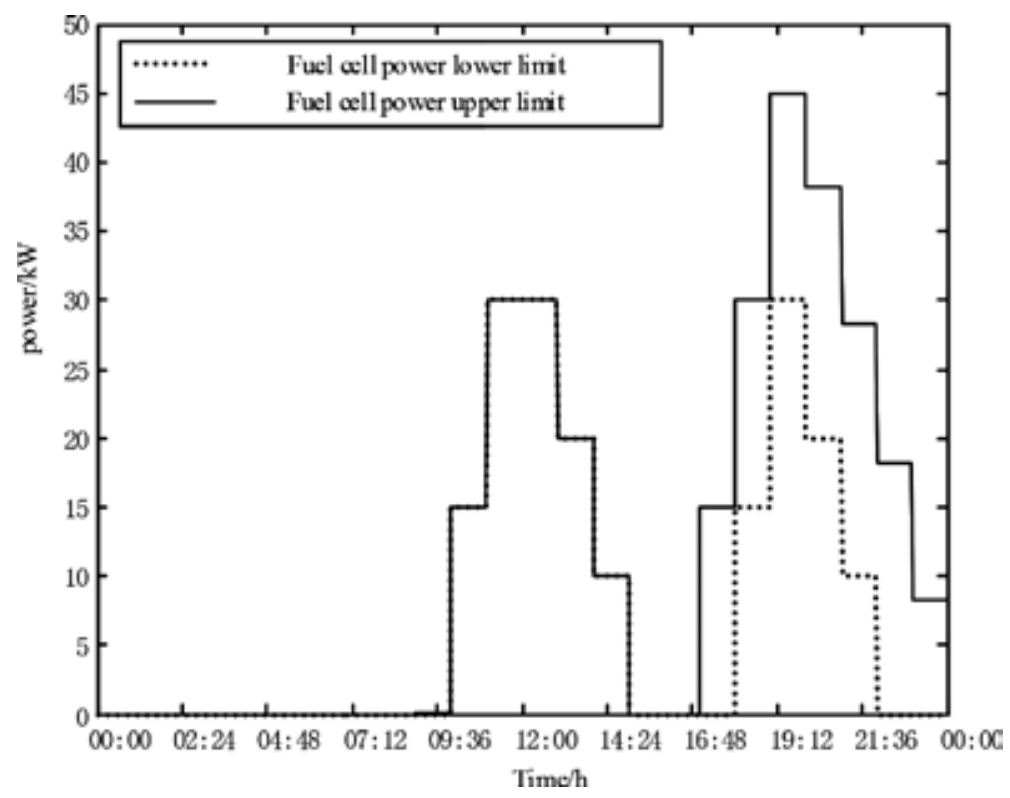

Figure 16. Fuel cell power intervals corresponding to $0 \%$ of load fluctuation.

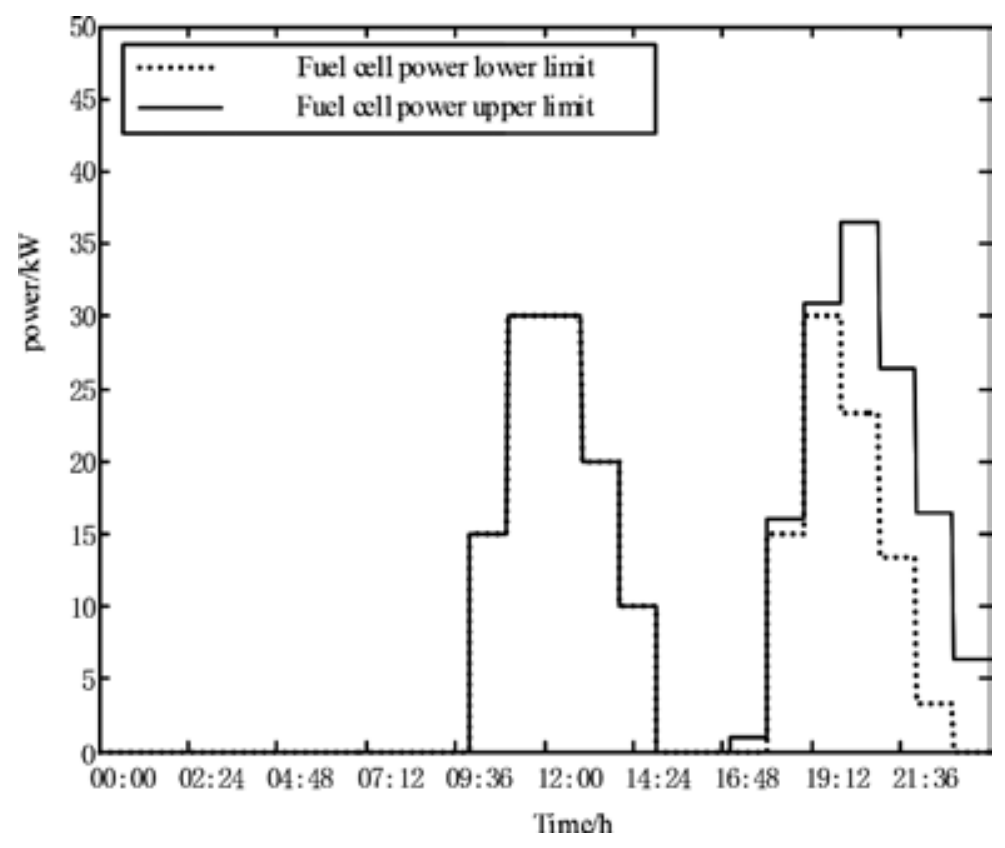

Figure 17. Fuel cell power intervals corresponding to $20 \%$ of load fluctuation. 


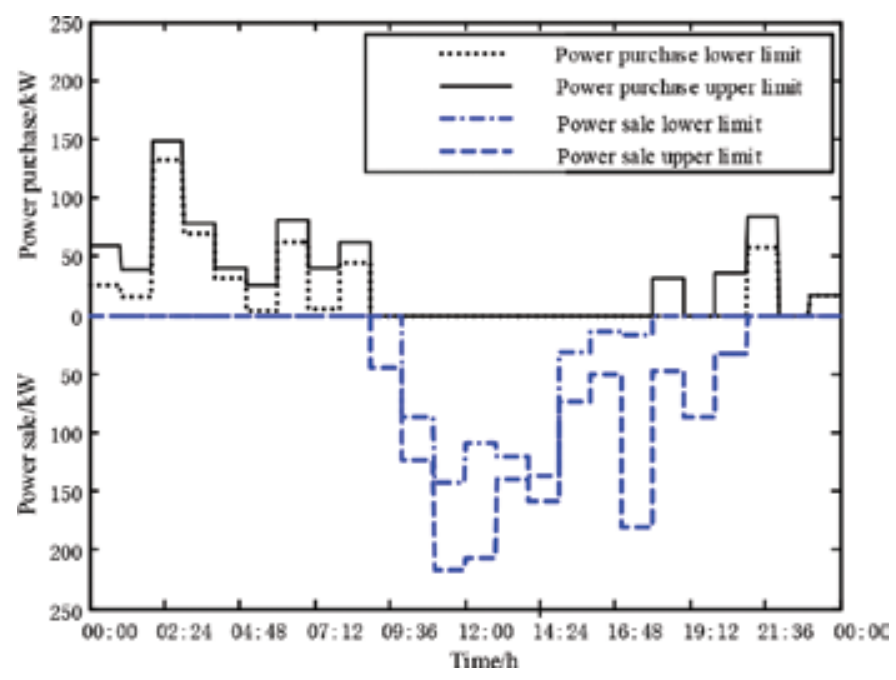

Figure 18. Power purchase and sale intervals corresponding to $0 \%$ of load fluctuation.

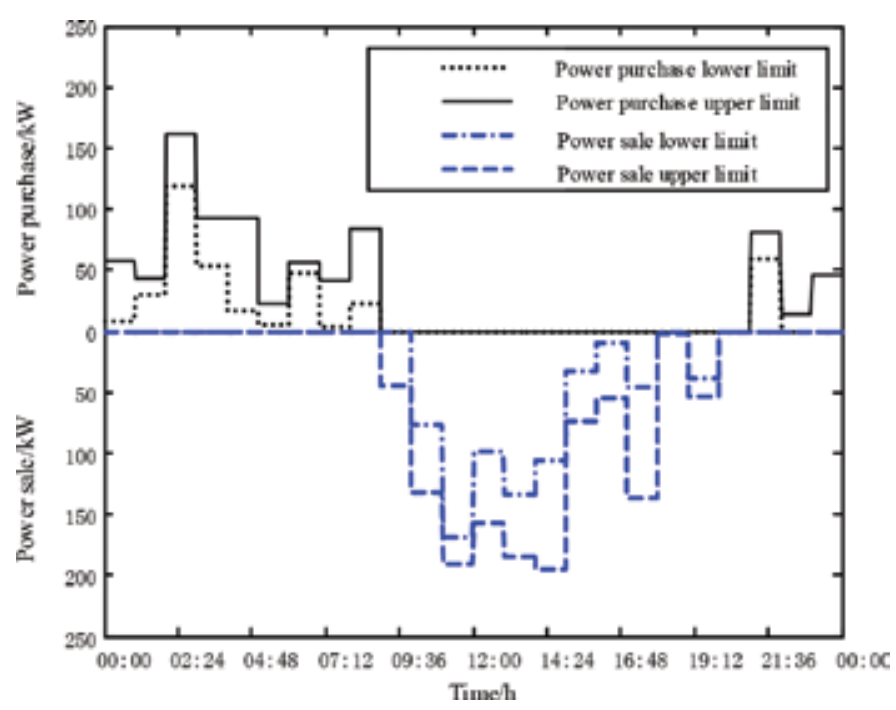

Figure 19. Power purchase and sale intervals corresponding to $20 \%$ of load fluctuation.

During load peak and normal periods, generation cost and discharging cost are lower than power purchase cost, and they still do not reach their maximum output, so generation unit can decide whether to generate more power and sale it to grid compared with the power sale price in order to obtain the optimal economic operation. During load peak periods (9:00-14:00 and 18:00-20:00), load fluctuation is relieved by the adjustment of storage batteries and fuel cells, and power purchase is not affected largely. During load normal periods (15:00-17:00), storage batteries are in charging condition and microgrid sale power, because WTs and PVs can supply 
load and microgrid has extra power. The extra power of microgrid is stored in storage batteries and sold to grid, so different levels of load fluctuation has larger impact on power sale for storage batteries during load normal periods.

\section{Conclusions}

In this chapter, the characteristics of DGs and ESSs are studied and the mathematical models are established specifically. Different operational control strategies of microgrid under different operation modes are analyzed and an economic operation optimization method is proposed considering uncertainties of DGs in microgrid. The effects of the uncertainty property of DERs and user loads in microgrid on the economic optimization of microgrid are demonstrated. In order to characterize these uncertainties in microgrid, the randomness and intermittent of the wind speed and solar radiation intensity are described in interval forms and then the output prediction interval values of the wind turbines and PVs are obtained. Based on the interval model of load uncertainty, a microgrid economic optimization model based on interval optimization method is proposed. Meanwhile, combined with the time-ofuse characteristic, issue of the power exchange with the external grid has been considered. Taking the effects analysis of ESSs on the economic operation of the system as an example, this chapter discusses the impacts of uncertainty of renewable energy power and load power on optimization results, as well as the effects of the degree of load uncertainty or load fluctuation on scheduling results. The results verify the robustness of the proposed method and model, and show the effectiveness in dealing with uncertainty optimization problem.

\section{Author details}

Shouxiang Wang ${ }^{1 *}$, Leijiao Ge ${ }^{1}$, Kai Wang ${ }^{1}$ and Shengxia Cai ${ }^{2}$

*Address all correspondence to: sxwang@tju.edu.cn

1 Key Laboratory of Smart Grid of Ministry of Education, Tianjin University, Nankai District, Tianjin, China

2 Zhou Enlai School of Goverment, Nankai University, Nankai District, Tianjin, China

\section{References}

[1] S. Wang, Z. Li, L. Wu, M. Shahidehpour, Z. Li. New metrics for assessing the reliability and economics of microgrids in distribution system. IEEE Transactions on Power Systems. 2013,28(3):2852-2861. 
[2] A.G. Tsikalakis, N.D. Hatziargyriou. Centralized control for optimizing microgrids operation. IEEE Transactions on Energy Conversion. 2008,23(1):241-248.

[3] S.-J. Ahn, S.-I. Moon. Economic scheduling of distributed generators in a micro-grid considering various constraints. Proceedings of the 2009 IEEE PES General Meeting, Calgary, 2009, 26-30.

[4] R. Karki, P. Hu, R. Billinton. A simplified wind power generation model for reliability evaluation. IEEE Transactions on Energy Conversion. 2006,21(2):533-540.

[5] T.-Y. Lee. Operating schedule of a battery energy storage system in a renewable energy based time-of-use rate industrial user with wind turbine generators: a multipass iteration particle swarm optimization approach. IEEE Transactions on Energy Conversion. 2007,22(3):774-782.

[6] S. Wang, L. Han, L. Wu. Uncertainty tracing for distributed generations via complex affine arithmetic based unbalanced three-phase power flow. IEEE Transaction on Power System. 2015,30(6):3053-3062.

[7] S. Wang, L. Han, P. Zhang. Affine arithmetic-based DC power flow for automatic contingency selection with consideration of load and generation uncertainties. Electric Power Components and Systems. 2014,42(8):852-860.

[8] Tong S. Interval number and fuzzy number linear programming. Fuzzy Sets and Systems. 1994,66:301-306.

[9] S-J. Ahn, S-I. Moon. Economic scheduling of distributed generators in a micro-grid considering various constraints. Proceedings of the 2009 IEEE PES General Meeting, Calgary. 2009, 26-30.

[10] S. Wang, X. Fan, L. Han, L. Ge. Improved interval optimization method based on differential evolution for microgrid economic dispatch. Electric Power Components and Systems. 2015,43(16):1882-1890. 

